\title{
Rogue Seasonality Detection in Supply Chains
}

\author{
by
}

\author{
Vinaya Shukla
}

A thesis submitted in partial fulfilment of the requirements for the degree of Doctor of Philosophy in Cardiff University

Logistics Systems Dynamics Group

Logistics and Operations Management Section

Cardiff Business School, Cardiff University

March 2010 
UMI Number: U584427

All rights reserved

\section{INFORMATION TO ALL USERS}

The quality of this reproduction is dependent upon the quality of the copy submitted.

In the unlikely event that the author did not send a complete manuscript and there are missing pages, these will be noted. Also, if material had to be removed, a note will indicate the deletion.

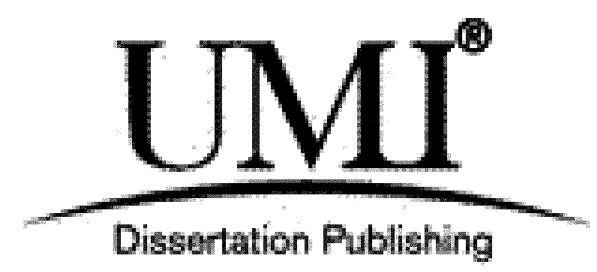

UMI U584427

Published by ProQuest LLC 2013. Copyright in the Dissertation held by the Author.

Microform Edition (c) ProQuest LLC.

All rights reserved. This work is protected against unauthorized copying under Title 17, United States Code.

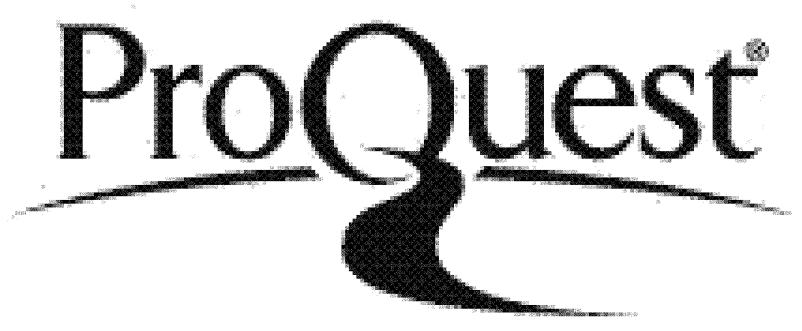

ProQuest LLC

789 East Eisenhower Parkway

P.O. Box 1346

Ann Arbor, MI 48106-1346 


\section{DECLARATION}

This work has not previously been accepted in substance for any degree and is not concurrently submitted in candidature for any degree.

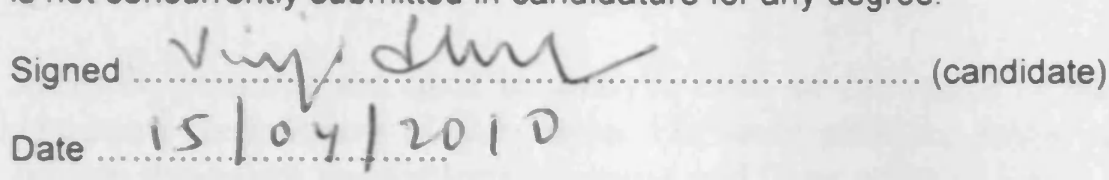

\section{STATEMENT 1}

This thesis is being submitted in partial fulfillment of the requirements for the degree of ......P. . A ........... (insert MCh, Md, MPhil, PhD etc, as appropriate)

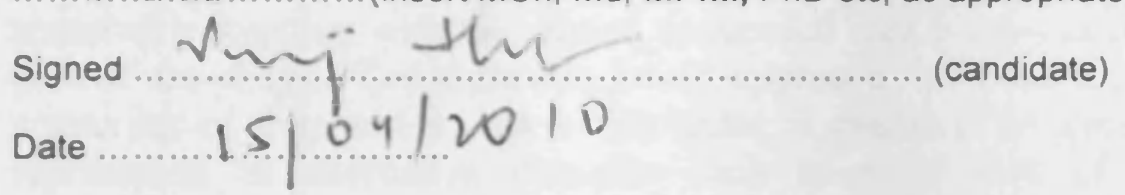

\section{STATEMENT 2}

This thesis is the result of my own independent work/investigation, except where otherwise stated.

Other sources are acknowledged by footnotes giving explicit references.

Signed
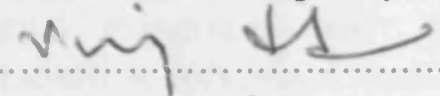
(candidate)

Date

$$
151.0412010
$$

\section{STATEMENT 3}

I hereby give consent for my thesis, if accepted, to be available for photocopying and for interlibrary loan, and for the title and summary to be made available to outside organisations.

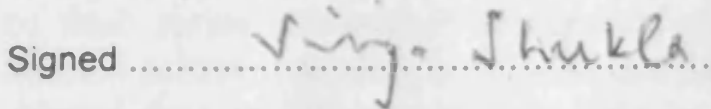
(candidate)

Date

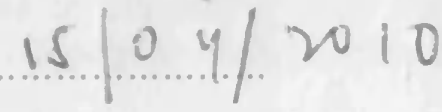




\section{Abstract}

Supply chains face disturbances in the provision of goods and services to customers. A key disturbance which is endogenously generated from the nature of the ordering process used is rogue seasonality, which is characterised by orders and other supply chain variables showing cyclicality in their profiles and this cyclicality not present in exogenous demand. It is observed in many supply chains and is a cause of significant economic loss for entities in these chains. A useful way to manage rogue seasonality could be by detecting its presence and intensity in a system and then taking appropriate and timely action for its mitigation. Called "sense and respond", this approach has been used in various domains extensively, but its application in supply chain management has been limited. This thesis explores the application of this approach for managing rogue seasonality, with the findings from it particularly relevant for a context where many multiple echelon supply chains are being managed by a focal company.

Multiple methods are used to analyse each of the rogue seasonality generation and detection components of the thesis. For understanding rogue seasonality generation, system dynamics simulations of single and three echelon linear and four echelon nonlinear (Beer game) systems are used. The impact of different demand processes and parameters, delays, order of delays, ordering processes, backlogs and batching on rogue seasonality is assessed. The simulation analysis is supported with empirical contexts from the steel and grocery sectors. The understanding gained on rogue seasonality together with the related contextual data is used in the sense or detection part of the thesis. The signature based approach, with the signature derived from clustering of time series data of variables is explored for detection, with the data represented in alternative domains such as amplitudes of Fourier transforms, autocorrelation function, coefficients of autoregressive model, cross correlation function and coefficients of discrete wavelet transform.

The thesis determined the signature and index for detecting rogue seasonality. While the signature, which is based on the cluster profiles of the system variables indicates the presence of rogue seasonality, the intensity of rogue seasonality is indicated by the index. In a multi supply chain context, the index could be used to identify problematic supply chains from a rogue seasonality perspective and initiate appropriate management action. At present there is no measure for rogue seasonality and defining an index for the same constitutes a major contribution of this thesis. Among alternative time series representations, the frequency domain representation based on Fourier transform was found to be the most appropriate for deriving the signature and index. This is also a major contribution of the thesis, as the comprehensive assessment of time series representations carried out in this study has not been done in many studies across domains, and those that have done so, have not used any supply chain related data in the assessment. Finally, the framework for exploiting the index for managing rogue seasonality is proposed. 


\section{Acknowledgements}

First and foremost, I would like to thank my primary supervisor Professor Mohamed Naim for his patience, support and encouragement throughout the PhD process. Thanks are also due to my second supervisor Dr Steve Disney for his valuable comments and insightful suggestions that helped in improving the quality of this research. I am also grateful to Professor Nina Thornhill from Imperial University for sharing her knowledge about some of the time series techniques that have been used in this thesis. I also wish to thank the following:

The Engineering and Physical Sciences Research Council (EPSRC) for funding this PhD through the Innovative Manufacturing Research Centre (IMRC) at Cardiff University.

My examiners Professor Joakim Wikner, Jonkoping University, Sweden and Professor Duc Truong Pham, Manufacturing Engineering Centre, Cardiff University

The administration and technical support especially Elaine, Elsie, Lainey, Philip Webber and Wayne Findlay.

My friends and colleagues within the Logistics and Operations management section for providing a productive and fun environment for doing this research. These include Wan, Noorfa, Hyunmi, Lourdes, Gul, Suhan, Champ, Polin, Poti, Vasco, Nazrul, Hartanto and Gilbert.

Last but not the least I wish to thank my family, Rachana and Shlesha for their support and understanding during the $\mathrm{PhD}$ journey. I am also extremely grateful to my parents especially my mother Krishna Shukla who always strived for us (me and my siblings) to have the best possible education and sacrificed a lot in the process. 


\section{Contents}

Chapter 1 Introduction.....................................................................................................................................................1

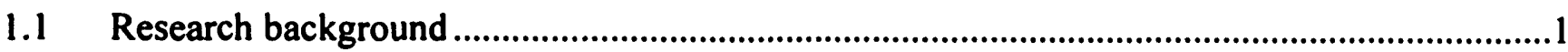

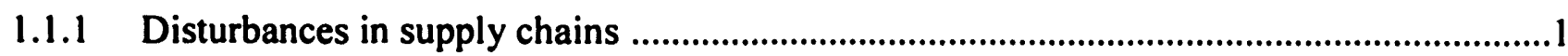

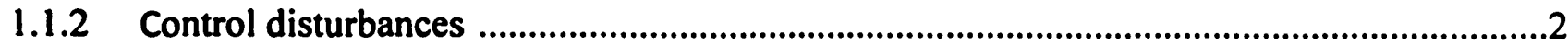

1.1.3 Sense and respond based approach to manage rogue seasonality ..........................................2

1.1.4 Signature based approach for detection .....................................................................

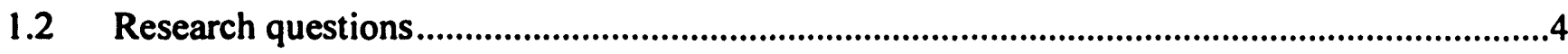

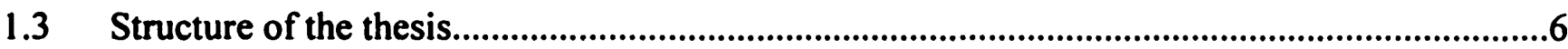

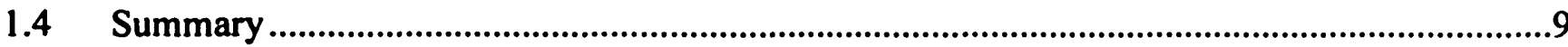

Chapter 2 Literature Review ...........................................................................................................10

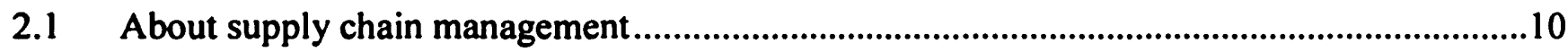

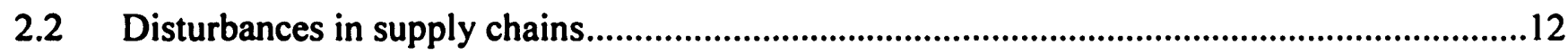

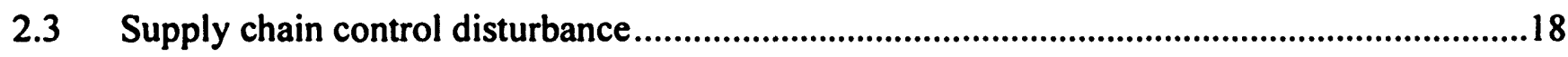

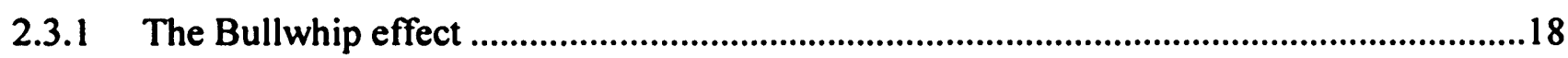

2.3.1.1 Causes of the Bullwhip effect ...................................................................................19

2.3.1.2 Methods used to study the Bullwhip effect.................................................................20

2.3.1.3 Measurement of the Bullwhip effect.............................................................................22

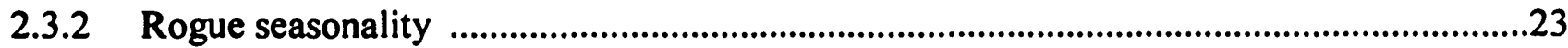

2.3.2.1 Previous research on rogue seasonality .................................................................24

2.3.3 Issues with design based approaches to manage control disturbances ..............................28

2.3.3.1 Complexity in supply chains.....................................................................................28

2.3.3.2 Irrationality in decision making ............................................................................30

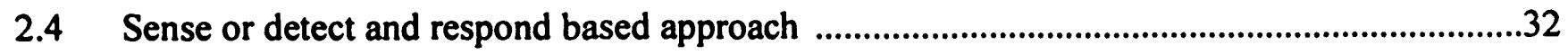

2.4.1 Status of detection based approaches in supply chain management ...................................34

2.4.2 Supply chain information in the context of detection .........................................................37

2.4.3 Generic approaches and techniques of detection ................................................................3

2.4.3.1 Change point detection techniques ............................................................................38

2.4.3.2 Signature based techniques ..........................................................................................39 


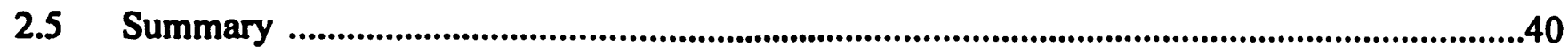

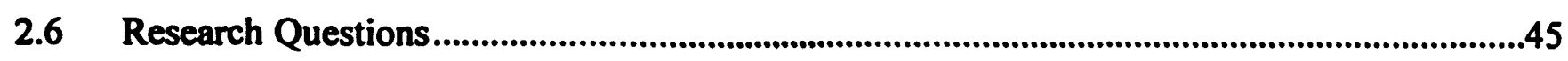

Chapter 3 Methodology

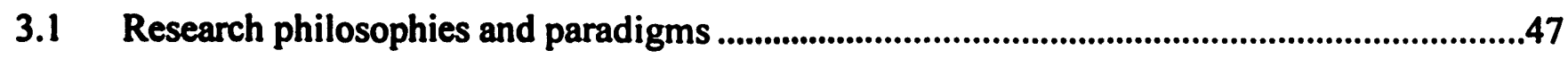

3.2 Nature of research in supply chain management ............................................................48

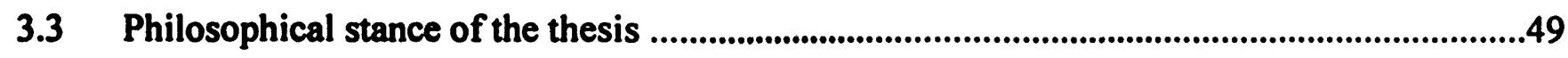

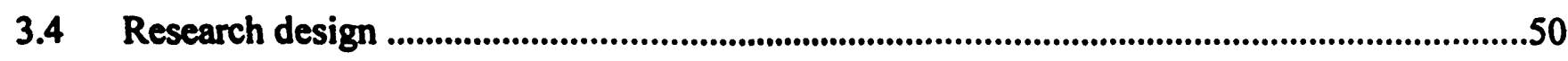

3.5 Literature review process ...............................................................................................51

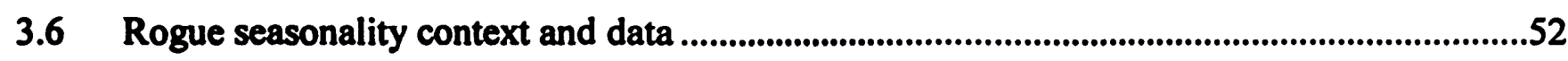

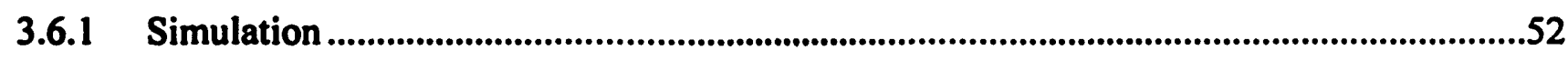

3.6.1.1 Discrete event simulation (DES) ..........................................................................53

3.6.1.2 System dynamics (SD) simulation ....................................................................54

3.6.1.3 Control theory and its use in production-inventory problems ....................................56

3.6.1.4 Simulation as applied in the thesis ....................................................................................63

3.6.1.5 Tools and techniques used ...........................................................................................66

3.6.2 Case studies...............................................................................................................................71

3.7 Time series transformations and features used for rogue seasonality detection........................72

3.8 Clustering and rogue seasonality signature.................................................................................81

3.9 Index of rogue seasonality ..........................................................................................................83

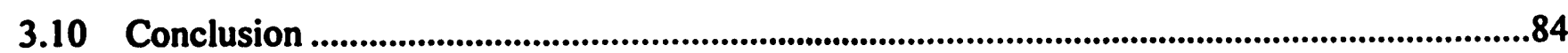

Chapter 4 Analysis of rogue seasonality in a linear supply chain system............................................85

4.1 Rogue seasonality in single echelon systems.............................................................................85

4.1.1 Control theory (frequency response) analysis....................................................................87

4.1.1.1 Frequency response analysis of a MTO system............................................................88

4.1.1.2 Frequency response analysis of a MTS system ..........................................................90

4.1.1.3 Evolution of rogue seasonality signature .....................................................................91

4.1.2 Simulation output analysis ...............................................................................................93

4.1.2.1 Simulation output analysis of MTO systems ...............................................................93

4.1.2.2 Simulation output analysis of MTS systems ...............................................................96

4.1.3 Index of rogue seasonality .................................................................................................98 
4.2 Rogue seasonality signature and index assessment in three echelon MTS systems with different time series transformations and features

4.3 Detailed simulation of three echelon MTS systems _......................................................104

4.3.1 Generation of rogue seasonality of different characteristics and intensities.....................104

4.3.2 Different features assessed for effectiveness in rogue seasonality index computation ..106

4.3.3 Different rogue seasonality index definitions considered .110

4.4 Analysis of output from detailed simulation of three echelon MTS system 110

4.4.1 Case I - Exogenous demand without seasonality and all variables used 111

4.4.1.1 General observations .111

4.4.1.2 Criteria for structured comparison of feature parameter combinations 113

4.4.1.3 Comparison of feature parameter combinations based on defined criteria. 114

4.4.1.4 Comparison of alternative index definitions 118

4.4.2 Case II - Exogenous demand with seasonality and all variables used 118

4.4.3 Case III - Exogenous demand without seasonality and only order and inventory variables used

4.5 Conclusions 124

Chapter 5 Analysis of rogue seasonality in a non-linear supply chain system 126

5.1 Description of the non- linear (Beer game) supply chain system. 127

5.2 Frequency response analysis of a hybrid MTO-MTS system. 129

5.3 System dynamics simulation of the Beer game system 132

5.4 Verification of Beer game simulation and rogue seasonality analysis 132

5.4.1 Verification of Beer game simulation by frequency response analysis............................134

5.4.2 Verification of Beer game simulation by time domain analysis......................................136

5.4.3 Rogue seasonality signature and index assessment .......................................................139

5.5 Detailed simulation of the Beer game system .......................................................................143

5.6 Analysis of output from detailed simulation of the Beer game system ................................145

5.6.1 Case IV - All variables used in index computation .........................................................145

5.6.1.1 Understanding the impact of different factors on rogue seasonality .........................146

5.6.1.2 Analysis of index values based on alternative feature parameter combinations .......147

5.6.1.3 Comparison of alternative index definitions...............................................................151 
5.6.2 Case V - Only order and inventory variables used in index computation. 151

5.7 Conclusions .152

Chapter 6 Analysis of rogue seasonality in empirical contexts. .155

6.1 Description of the steel case study context and its analysis based on the spectral principal component technique .156

6.2 Rogue seasonality analysis of the steel case study based on signature and index 159

6.2.1 Analysis of rogue seasonality signature derived from different features .161

6.2.2 Structured comparison of feature parameter combinations for index computation.......166

6.2.3 Comparison of alternative index definitions .................................................................169

6.3 Discussion of the steel case study findings ……...............................................................170

6.4 Description of the grocery case study context .................................................................171

6.5 Rogue seasonality analysis of the grocery case study based on signature and index .............173

6.5.1 Analysis of rogue seasonality signature derived from different features .........................175

6.5.2 Structured comparison of feature parameter combinations for index computation........181

6.5.3 Comparison of alternative index definitions .................................................................183

6.6 Discussion of the grocery case study findings ……..........................................................183

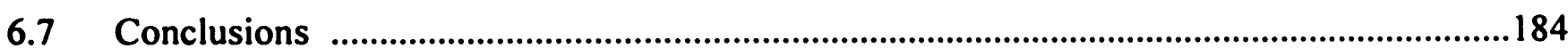

Chapter 7 Discussion and Conclusions ..............................................................................................186

7.1 Research background and process .............................................................................186

7.2 Discussion on research questions.................................................................................187

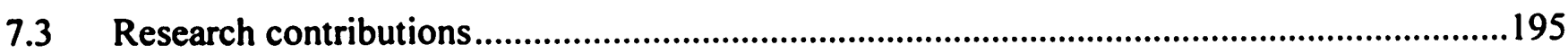

7.4 Limitations of the research and suggestions for further study .............................................197

References

\section{Appendices}

A Block diagram and difference equations for a single echelon APIOBPCS system .213

B Transfer functions for single echelon APIOBPCS (hybrid MTO-MTS), make to order (MTO) and make to stock (MTS) systems

C Theoretical spectral density plots for different AR and MA time series processes 
D Matlab code for different time series transformations and use of relevant features for computation of the rogue seasonality index.

E Average and coefficient of variation of rogue seasonality index values for a 3 echelon MTS system based on different feature parameter combinations; All variables used in the index computation

F Average and coefficient of variation of rogue seasonality index values for a 3 echelon MTS system based on different feature parameter combinations; Only order and inventory

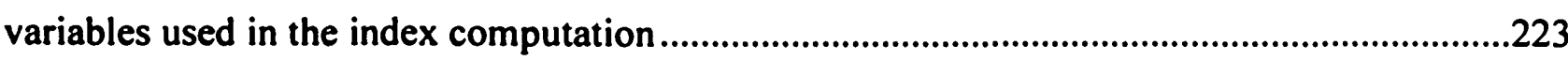

G Sample of Matlab code used for different tasks in Beer game system analysis .225

H Frequency response (FR) of select variables computed from Beer game simulation outputs H.1 Different lead times .227

H.2 Different batch sizes .228

I Index values for the Beer game system based on different feature parameter combinations: All variables used in the index computation

I.1 Average index values .229

I.2 Coefficient of variation of index values .230

J Index values for Beer game system based on different feature parameter combinations: Only order and inventory variables used in the index computation

J.1 Average index values .231

J.2 Coefficient of variation of index values .232

J.3 Structured evaluation of feature parameter combinations used for index computation .233

J.4 Correlation between alternative rogue seasonality indices .233 


\section{List of Figures}

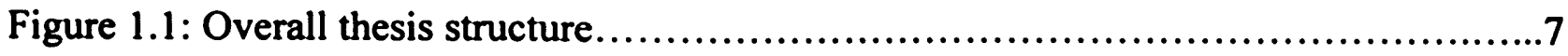

Figure 2.1: Rogue seasonality generation in different empirical contexts................. 25

Figure 2.2: Decision making loops related to sense and respond approach..................32

Figure 2.3: Benefits of automatic disturbance detection in a supply chain context............35

Figure 2.4: Generic disturbance detection techniques..................................38

Figure 3.1: Research framework used in the thesis.........................................50

Figure 3.2: Causal loop diagram of the APIOBPCS model.................................60

Figure 3.3: Screen shot of the VBA based APIOBPCS simulator used........................68

Figure 3.4: Screen shot of the VBA based Beer game simulator used.......................69

Figure 3.5: Profiles of some commonly used wavelets...............................76

Figure 4.1: Time series, spectra and their clustering for single echelon MTO and MTS systems excited by Gaussian demand (CONS) ................................94

Figure 4.2: Plots and clusters using different features for a three echelon MTS system with delay order one excited by Gaussian demand.............................100

Figure 4.3: Plots and clusters using different features for a three echelon MTS system with delay order infinity excited by Gaussian demand.

Figure 4.4: Variation in rogue seasonality index from exogenous demand having an additional seasonal component (seasonal component is notch filtered before index computation) .......................................................120

Figure 5.1: Schematic of the Beer game supply chain system...........................127

Figure 5.2: Comparison of frequency response derived analytically for a four echelon linear supply chain with that computed from the Beer game simulation output.........135

Figure 5.3: Time series profiles of variables from a simulated Beer game system under different settings subjected to a step change in demand.........................138

Figure 5.4: Impact of ordering practices on the signature and index of rogue seasonality based on FT (Total) feature parameter combination.

Figure 6.1: Steel case study network, its constituent dyads and the variables used in analyzing them

Figure 6.2: Profiles of variables in the frequency domain and clustering based on these profiles for the three dyads in the supply network 160 
Figure 6.3: Normalised time series profiles and clustering based on these profiles for

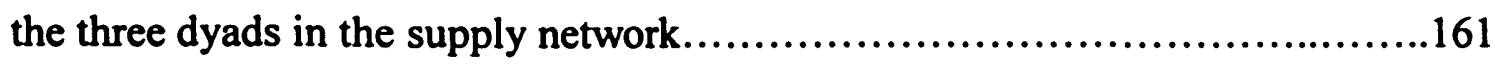

Figure 6.4: Autocorrelation function (ACF) profiles and clustering based on these profiles for the three dyads in the supply network................................162

Figure 6.5: Cross correlation between variables and clustering based on it for the three dyads in the supply network

Figure 6.6: Grocery case study network structure and the variables used for the rogue

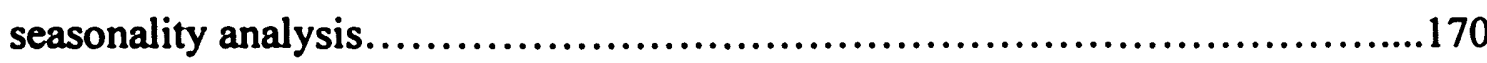

Figure 6.7: Profiles and clustering using different features for grocery case study product with manufacturing induced rogue seasonality (SKU 1) ..................174

Figure 6.8: Profiles and clustering using different features for grocery case study product without manufacturing induced rogue seasonality (SKU 2)...............175

Figure 6.9: Profiles and clustering using different features for grocery case study product without manufacturing induced rogue seasonality (SKU 3).............176

Figure 6.10: Profiles and clustering using different features for grocery case study product without manufacturing induced rogue seasonality (SKU 4).

Figure 7.1: Flow chart for the practical application of the rogue seasonality index. 


\section{List of Tables}

Table 2.1: Select studies on Supply Chain Disturbances.........................................14

Table 2.2: Application of detection based approach in different domains............................33

Table 3.1: Research methods applied in logistics and supply chain management....................49

Table 3.2: Simulation design used for rogue seasonality analysis...............................63

Table 3.3: Studies which have used Beer game simulation.......................................64

Table 3.4: Review of studies using different transformation techniques and features in time

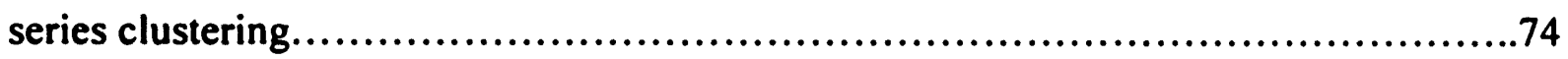

Table 4.1: Frequency response (FR) functions of variables in make to order (MTO) and make

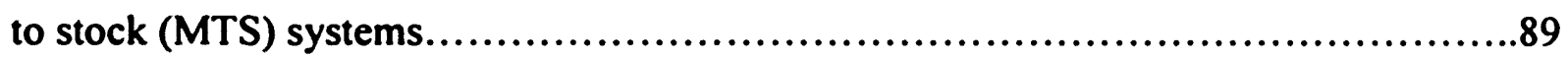

Table 4.2: Simulation design for generating rogue seasonality of different characteristics and

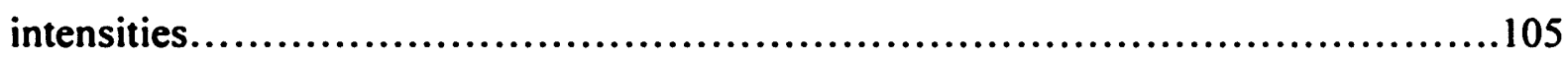

Table 4.3: Time series transformation techniques, features and parameters for each feature used for rogue seasonality index computation.

Table 4.4: Rogue seasonality index for a three echelon MTS system based on different feature parameter combinations; Demand process: AR (1); All variables used..................112

Table 4.5: Structured evaluation of feature parameter combinations used for rogue seasonality index computation in a three echelon MTS system; All variables used..................115

Table 4.6: Correlation coefficient between alternative rogue seasonality indices in a linear three echelon MTS system - All variables used in computation of the indices.

Table 4.7: Rogue seasonality index of a three echelon MTS system based on different feature parameter combinations; Demand process: AR (1); Only order and inventory variables used

Table 4.8: Structured evaluation of feature parameter combinations used for rogue seasonality index computation in a three echelon MTS system; Only order and inventory vaiables used.

Table 4.9: Correlation coefficient between alternative rogue seasonality indices in a linear three echelon MTS system - Only orders and inventory variables used

Table 5.1: Frequency response (FR) functions of variables at select points for hybrid MTO-MTS systems. 
Table 5.2: Difference equations used to simulate the Beer game supply chain system

Table 5.3: Simulation design for generating rogue seasonality of different characteristics and intensities in the Beer game system.

Table 5.4: Structured evaluation of different feature parameter combinations for rogue seasonality index computation in the Beer game system; All variables used.

Table 5.5: Correlation between alternative rogue seasonality indices in the Beer game system - All variables used in computation of the indices

Table 6.1: Assessment of select feature parameter combinations used for rogue seasonality index computation in the steel case study. 166

Table 6.2: Correlation between alternative rogue seasonality indices for the steel case study data.

Table 6.3: Evaluation of different feature parameter combinations used for rogue seasonality index computation in the grocery case study.

Table 6.4: Correlation between alternative rogue seasonality indices for the grocery case study data. 
List of Abbreviations and Symbols

\begin{tabular}{|c|c|}
\hline $\begin{array}{l}\text { Abbreviation } \\
\text { /Symbol }\end{array}$ & Description \\
\hline $\mathrm{ACF}$ & $\begin{array}{l}\text { Auto Correlation Function - Correlation of a dataset or time series } \\
\text { with itself offset by } 1, \ldots . n \text { lags or time periods. The numerical } \\
\text { suffix with ACF used in the thesis, refers to number of lags }\end{array}$ \\
\hline AINV & $\begin{array}{l}\text { Actual INVentory - The actual inventory of goods at the echelon } \\
\text { in context }\end{array}$ \\
\hline Amp R & $\begin{array}{l}\text { Amplitude Response - The amplitude gain (ratio of output to input } \\
\text { sinusoid amplitude) of the system at each frequency }\end{array}$ \\
\hline APIOBPCS & $\begin{array}{l}\text { Automatic Pipeline, Inventory and Order based Production Control } \\
\text { System - Generic structure of the ordering decision used in this } \\
\text { thesis and which has three components: Work in progress or orders } \\
\text { placed but not yet received feedback loop (automatic pipeline), the } \\
\text { inventory feedback loop and the feed forward of the forecasted } \\
\text { sales (orders) }\end{array}$ \\
\hline AR model & $\begin{array}{l}\text { Autoregressive model - It is a linear predictive model of a time } \\
\text { series where the value at a particular time is a function of its values } \\
\text { in the previous time periods. The number of past time periods used } \\
\text { is referred to as the order of the model and is represented as a } \\
\text { suffix with AR or AR model in the thesis }\end{array}$ \\
\hline AIC & $\begin{array}{l}\text { Akaike's Information Criterion - It is a criterion used for selecting } \\
\text { a model (among competing models) and involves trading off the } \\
\text { model's complexity (measured by number of parameters in the } \\
\text { model) and the model fit (measured by sum of squared residuals) }\end{array}$ \\
\hline AVCONS & $\begin{array}{l}\text { AVerage CONSumption - A forecast generated by the echelon in } \\
\text { question and which is a component in the ordering decision }\end{array}$ \\
\hline CCF & $\begin{array}{l}\text { Cross Correlation Function - Correlation between two datasets or } \\
\text { time series offset by } 1, \ldots . n \text { lags. The numerical suffix with CCF, } \\
\text { used in the thesis, refers to number of lags }( \pm) \text { considered }\end{array}$ \\
\hline COMRATE & $\begin{array}{l}\text { COMpletion rate - The rate of receipts of goods (against orders } \\
\text { placed earlier) which could be from external suppliers or internally } \\
\text { from production }\end{array}$ \\
\hline CONS & $\begin{array}{l}\text { CONSumption - The demand rate or sales of the system under } \\
\text { study. This could be at the end customer or other intermediate } \\
\text { entities in the supply chain }\end{array}$ \\
\hline DINV & Desired INVentory - Refers to the desired inventory at an echelon \\
\hline DWIP & $\begin{array}{l}\text { Desired Work in Progress - The desired or expected WIP going } \\
\text { through an echelon in the supply chain and which is computed by } \\
\text { multiplying the best estimate of production/distribution lead time } \\
\text { with the average demand }\end{array}$ \\
\hline DWT & $\begin{array}{l}\text { Discrete Wavelet Transform - Involves transformation of the data } \\
\text { set/time series into an alternate domain represented by the wavelet } \\
\text { basis functions and where the wavelet scales and positions (used in } \\
\text { the transformation) are based on powers of two. The numerical } \\
\text { suffix with DWT, used in the thesis, reflects the level of } \\
\text { decomposition of the signal }\end{array}$ \\
\hline
\end{tabular}




\begin{tabular}{|c|c|}
\hline $\begin{array}{l}\text { Abbreviation } \\
\text { /Symbol }\end{array}$ & Description \\
\hline EINV & $\begin{array}{l}\text { Error in Inventory - The difference between the desired or target } \\
\text { inventory level (DINV) and the actual level of inventory (AINV) }\end{array}$ \\
\hline EWIP & $\begin{array}{l}\text { Error in Work in Progress - The difference between the desired } \\
\text { work in progress (DWIP) and the actual WIP levels. }\end{array}$ \\
\hline$f$ & $\begin{array}{l}\text { Frequency of a sinusoidal signal expressed in units of cycles per } \\
\text { time period }\end{array}$ \\
\hline FR & $\begin{array}{l}\text { Frequency Response - It is a measure at various frequencies of a } \\
\text { system's output spectrum in response to an input signal and } \\
\text { consists of amplitude response (Amp R) and phase response }\end{array}$ \\
\hline FT & $\begin{array}{l}\text { Fourier Transform - Involves transformation of a time series into } \\
\text { its constituent sinusoids in terms of amplitude and phase of } \\
\text { different frequencies. The suffix with FT (as used in the thesis) } \\
\text { reflects the number of initial frequencies used for a subsequent } \\
\text { analysis after FT }\end{array}$ \\
\hline LT & $\begin{array}{l}\text { Lead time - The delay between order placement and receipt of } \\
\text { goods into inventory }\end{array}$ \\
\hline MA model & $\begin{array}{l}\text { Moving Average model - It is a linear regression of the current } \\
\text { value of the time series against previous (unobserved) white noise } \\
\text { error terms or random shocks. The number of previous error terms } \\
\text { used is referred to as the order of the model and is represented as a } \\
\text { suffix with MA in the thesis }\end{array}$ \\
\hline MTO & $\begin{array}{l}\text { Make to Order - Manufacturing strategy geared to satisfy customer } \\
\text { requirements only upon receipt of a customer's order. }\end{array}$ \\
\hline MTS & $\begin{array}{l}\text { Make to Stock - A build-ahead manufacturing strategy where } \\
\text { production plans are based upon sales forecasts and/or historical } \\
\text { demand; goods are produced and stocked in anticipation of } \\
\text { demand and customer demand is met from these stocks }\end{array}$ \\
\hline ORATE & Order Rate - The production or goods order rate \\
\hline PCA & $\begin{array}{l}\text { Principal component analysis - It is a mathematical procedure that } \\
\text { transforms a number of possibly correlated variables into a smaller } \\
\text { number of uncorrelated variables called principal components. The } \\
\text { original data is expressed in terms of the principal components for } \\
\text { further analysis }\end{array}$ \\
\hline $\boldsymbol{\theta}$ & $\begin{array}{l}\text { MA model parameters; As many parameters }\left(\theta_{1}, \ldots \ldots \theta_{n}\right) \text { are used as } \\
\text { the order of the model }\end{array}$ \\
\hline$\rho$ & $\begin{array}{l}\text { AR model parameters; As many parameters }\left(\rho_{1}, \ldots \ldots \rho_{n}\right) \text { are used as } \\
\text { the order of the model }\end{array}$ \\
\hline s & 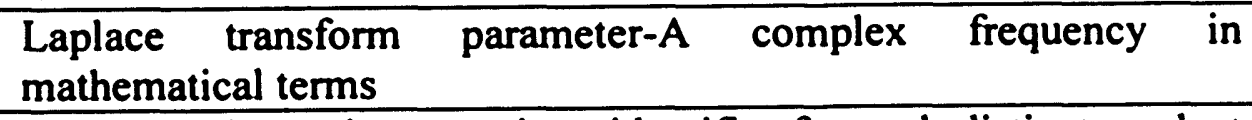 \\
\hline SKU & $\begin{array}{l}\text { Stock keeping unit - A unique identifier for each distinct product } \\
\text { which enables a company to systematically track its inventory or } \\
\text { availability }\end{array}$ \\
\hline SPCA & $\begin{array}{l}\text { Spectral principal component analysis - Principal component } \\
\text { analysis done on the spectra of the time series variables so that } \\
\text { time shifts in the data caused by delays and lags in the process } \\
\text { dynamics are eliminated }\end{array}$ \\
\hline
\end{tabular}




\begin{tabular}{|ll|}
\hline $\begin{array}{l}\text { Abbreviation } \\
\text { /Symbol }\end{array}$ & Description \\
\hline $\mathrm{T}_{\mathbf{a}}$ & $\begin{array}{l}\text { Time to average consumption - A constant used in the forecasting } \\
\text { mechanism that defines how "fast" a signal is followed. It is the } \\
\text { average age of the forecast }\end{array}$ \\
\hline $\mathrm{T}_{\mathrm{i}}$ & $\begin{array}{l}\text { Time to adjust inventory - A constant that specifies the number of } \\
\text { time periods over which the error in inventory is adjusted }\end{array}$ \\
\hline $\mathrm{T}_{\mathrm{p}}$ & $\begin{array}{l}\text { The actual lead time between placement and receipt of order. It is } \\
\text { the same as LT }\end{array}$ \\
\hline $\mathrm{T}_{w}$ & $\begin{array}{l}\text { Time to adjust work in progress or WIP - A constant that specifies } \\
\text { the number of time periods over which the error in work in } \\
\text { progress is adjusted }\end{array}$ \\
\hline WIP & $\begin{array}{l}\text { Work in progress - The volume of products that have been ordered } \\
\text { but not yet received }\end{array}$ \\
\hline$\omega$ & $\begin{array}{l}\text { Angular frequency - Frequency of a sinusoidal signal expressed in } \\
\text { units of radians per time period. }\end{array}$ \\
\hline$\omega$ max FR & $\begin{array}{l}\text { Frequency which corresponds to the maximum frequency response } \\
\text { value }\end{array}$ \\
\hline$\omega$ Crossover & $\begin{array}{l}\text { Frequency which corresponds to transition of the FR profile from } \\
\text { amplification to attenuation i.e. where FR value is equal to 1 }\end{array}$ \\
\hline
\end{tabular}




\section{Chapter 1 - Introduction}

This chapter presents the background and motivation of the research. The main themes are explained in brief and then collated to formulate the research questions. This is followed by a description of the structure of the thesis.

\section{$1.1 \quad$ Research background}

The research in this thesis has been conducted as a part of the five-year Mass Customised Collaborative Logistics for Sustainable Manufacturing (McCLOSM) flagship project of the Innovative Manufacturing Research Centre (IMRC) at Cardiff University. This project is sponsored by the Engineering and Physical Sciences Research Council (EPSRC) and was undertaken jointly with policy makers, trade bodies and a number of industrial partners from diverse sectors such as food, steel and construction. The author's role in the project was to look at how collaboration and information sharing can be used for the detection of disturbances and consequent mitigation of risks in a supply chain.

\subsubsection{Disturbances in supply chains}

Supply chains, which consist of all intermediate entities connecting producers and consumers such as vendors, manufacturers, distributors and retailers routinely face disturbances, which prevent them from realising their performance objectives (Davis, 1993). Defined as random or unexpected occurrences that deviate from 'normal' (Svensson, 2000), these disturbances could arise from customer demand, suppliers, internal manufacturing processes, environmental factors or control systems (Davis, 1993; Mason-Jones and Towill, 1998; Juttner, 2005). Amongst these, disturbances from control systems, which originate from use of inappropriate information and/or inappropriate ordering/control algorithms to match supply and demand, affect a large number of supply chains. Practitioners either passively accept them or ignore them, (Geary et. al, 2002; McCullen and Towill, 2002) both of which cause economic loss for the firms in the supply chain (Metters, 1997; Lee et al., 1997a, b). 


\subsubsection{Control disturbances}

Two kinds of control disturbances have been identified in the literature. One is the Bullwhip effect in which variability in orders is amplified as one moves from downstream to upstream echelons (Forrester, 1961; Lee et al., 1997a, b). The second is endogenous generation of seasonality in orders and other supply chain variables (Forrester, 1961; Kim and Springer, 2008). This seasonality, which is generated by a company's own internal processes such as inventory and production control systems, is not present in exogenous demand and is therefore also referred to as rogue seasonality (McCullen and Towill, 2002).

Extensive research has been conducted on the Bullwhip effect (Geary et al, 2006; Miragliotta, 2006) including its occurrence in different empirical sectors (Miragliotta, 2006), its multiple causes such as demand signal processing and lead time (Forrester effect), batching, shortage gaming, price promotion and behavioural (Geary et al., 2006) and various approaches for its measurement. Moreover, different methods such as statistical, control theory, system dynamics simulation and management games (specifically Beer game) have been used to study it. On the other hand, the literature highlights only two studies focussed on rogue seasonality (Thornhill and Naim, 2006; Kim and Springer, 2008). Rogue seasonality is present in many supply chains (Kaipia et al., 2006; Lee et al., 1997a; Torres and Moran, 2006) and its presence causes cost inefficiency. Costs to the extent of $10-20 \%$ could be reduced by elimination of such seasonal variations as per Metters (1997). The study of rogue seasonality is therefore important and forms the focus of this thesis.

\subsection{3 "Sense and respond" based approach to manage rogue seasonality}

One approach to manage rogue seasonality is to minimise its generation and intensity through appropriate choice of forecasting and ordering parameters. Kim and Springer (2008) used this approach to determine the conditions under which strong or weak seasonality could be endogenously generated in a dyadic system based on analytical system dynamics analysis. However, such an approach may not be very effective in real environments. This is because, supply chains in general are quite complex which makes them difficult to control (McCarthy and Tan, 2000; Baader and Montanus, 
2008). At the same time, optimal control policies and parameters established in theoretical studies are too simplistic in relation to this complex reality, having been developed under dyadic settings. Finally, there is additional uncertainty from the way these policies and parameters are applied in practice in real environments because of behavioural biases (Bendoly and Cotteleer, 2008; Croson et al., 2005). One alternative is to use the "sense and respond" based approach which is particularly suited for dynamic environments.

The "sense and respond" approach involves use of information to sense changes in the environment or context and then, based on it, initiating appropriate management response. This approach has been applied in different strategic and operating contexts, with its potential use also recognised in supply chain risk management by researchers such as Hendricks and Singhal (2003) and Craighead et al. (2007). The "sense and respond" based approach could potentially be used to manage rogue seasonality.

\subsubsection{Signature based approach for detection}

Among the various alternatives available for sensing or detection, the signature based approach was considered because of its flexibility in handling different profiles and numbers of variables as well as availability of a wider choice of techniques for application. Though application of this approach in the supply chain management domain has been limited, it has been successfully applied in various other domains such as finance, equipment maintenance, healthcare and computer systems (Edge and Sampai, 2009; Lee et al., 2006; Lamma et al., 2006; Zhu et. al., 2001).

Use of the signature based approach involves choosing the techniques for determining the signature or rule/s of detection as well as the nature of information to be fed to these techniques. Clustering is the most common technique for analyzing multiple time series data (Liao, 2005) and it was therefore used for signature formulation. Signatures based on clustering involve finding similarity or dissimilarity relationships among the time series profiles of the operating variables, allocating them to separate homogenous groups or clusters and relating the profile of clusters to a particular disturbance. 
In terms of nature of information used for formulating signatures, either raw time series could be used or it could be transformed to other domains, and features from these domains used. Typical features that have been applied in time series clustering studies in different disciplines are: the amplitudes of Fourier transforms (FT) (Agrawal et al., 1993), coefficients from an autoregressive (AR) model (Kalpakis et al., 1999), wavelet coefficients from discrete wavelet transform (DWT) (Chan and Fu, 1999), autocorrelation function (ACF) (Wang and Wang, 2000) and cross correlation function (CCF) which is a feature of pairs of time series (Bohte et al., 1980). However, two issues regarding these time series clustering studies stand out. Firstly, none of these studies used supply chain contextual data in their analysis. Since the effectiveness of feature/s is domain specific, findings from these studies have limited applicability for the supply chain as well as rogue seasonality contexts. Secondly, few studies have compared the performance of their features with other features, and those that have, have used only one or two features in the comparison (Keogh and Kasetty, 2003; Liao, 2005). The most appropriate time series transformation and feature for analyzing supply chains in general and rogue seasonality in particular, therefore, is an open question.

\subsection{Research questions}

To summarise, the study of rogue seasonality is important because it is present in many real world supply chains and is a cause of significant economic loss for entities in these chains. One way to minimise this loss is by detecting, diagnosing and discriminating rogue seasonality from exogenous disturbances and using it to improve the timeliness and quality of remedial management action. Christopher and Lee (2004) highlighted the need for intelligent alerts for out of control situations and the importance of detection based approaches in supply chains. Use of this approach for the detection of rogue seasonality was explored by Thornhill and Naim (2006) who proposed a lookup table based on spectra PCA (principal component analysis) for the same. However, this study was not comprehensive. Data from only a single case study was used and only a single detection technique (spectra PCA or SPCA) was tested. The technique also required significant manual intervention, which meant subjectivity in the detection process. A more objective and automated approach for rogue seasonality detection, is therefore required. Such a detection based approach would be 
especially useful in an environment where many multiple echelon supply chains are being managed by a focal company.

Use of the "sense and respond" approach is explored, which involves processing of system information to sense or detect rogue seasonality and then taking appropriate management action (respond) to reduce its intensity and thereby related economic loss. For detecting rogue seasonality, signature based approaches are tried with the signatures derived from clustering of time series information of system variables. The signature could be in the form of a subjective rule or an objective index which indicates the intensity of rogue seasonality. No such index measure exists for rogue seasonality at present, in contrast to the Bullwhip effect, for which multiple measures have been proposed. Establishment of the signature and index also provide the opportunity to automate the rogue seasonality detection process and in this way address one of the limitations of the study by Thornhill and Naim (2006).

The nature of system information, in terms of whether it is in the time or other domains such as amplitudes of FT, AR model parameters, DWT coefficients, ACF and $\mathrm{CCF}$ affects the effectiveness of the signature. Given that the best domain representation for clustering supply chain data is not established, all these alternative domains need to be tried to determine the same for the rogue seasonality signature. Finally, information on only a few rather than all the variables could be available, and therefore, it is important to assess sensitivity of the findings to the same.

An important consideration in this research is the rogue seasonality context to be used for assessing the effectiveness of different aspects of the detection process. While a simulated context provides controlled experimentation and in this way facilitates different rogue seasonality contexts to be assessed, real world examples of rogue seasonality help in increasing the external validity of the findings. When used together, they complement each other effectively. Use of multiple contexts increases the validity of the research findings on a generic basis also (Shukla et al., 2009; Boyer and Swink, 2008).

Putting together the various aspects of the detection process with the different rogue seasonality contexts, the following research questions emerge which this thesis seeks to answer: 
1) What signature and index could be used for detecting rogue seasonality in a linear supply chain? What is the best feature (domain representation) and the appropriate system variables for deriving them?

2) Can the signature and index identified from the linear supply chain be applied to a non-linear supply chain with backlogs and batching? Is the best feature and appropriate variables for detecting rogue seasonality in this non-linear system the same as in the linear system?

3) Can the signature and index be used for detecting rogue seasonality in real supply chains? Are the best features and system variables for deriving the signature and index the same as found in RQ1 and RQ2?

4) How can rogue seasonality detection be used in a managerial framework?

The linear supply chain system was considered as it is analytically tractable (Towill, 1982) and therefore yields exact solutions which could be used for a more accurate assessment of different aspects of the detection process. On the other hand the nonlinear supply chain with backlogs and batching is a more realistic system and it was therefore also analysed. Finally, the empirical rogue seasonality contexts used, add to the limited knowledge base of the same. The linear, non-linear and empirical contexts used are discussed in the Methodology chapter where they are best explained.

In terms of research questions, these establish the process of rogue seasonality detection in terms of the signature and index as indicators of rogue seasonality, appropriate system variables to be used and the domain in which they should be represented (as features) in each of linear, non-linear and empirical contexts individually. This multi context assessment of different aspects of detection increase the robustness of the findings with respect to the same. Finally, RQ 4 is aimed at using the detection facilitated by RQ 1, 2, 3 to improve the management of rogue seasonality in an unseen setting.

\subsection{Structure of the thesis}

This thesis is organised into seven chapters as shown in Figure 1.1.

Chapter 1 introduces the background and motivation for the research. Gaps in research are collated to form the research questions: 
Chapter 2 reviews the literature around the core research theme. Supply chain disturbances, control disturbances including Bullwhip and rogue seasonality, "sense or detect and respond" based approaches for rogue seasonality detection and applicability of signatures based on clustering of alternative domain representations of system variables are reviewed. A major part of this work is included in the presentation made at the $14^{\text {th }}$ International Annual EurOMA Conference in 2007 whose full reference is as follows:

Shukla, V. and Naim, M. M. (2007). "An operations management framework for disturbance detection in supply networks", Proceedings of the $14^{\text {th }}$ International Annual EurOMA Conference, June 17-20, Ankara, Turkey.

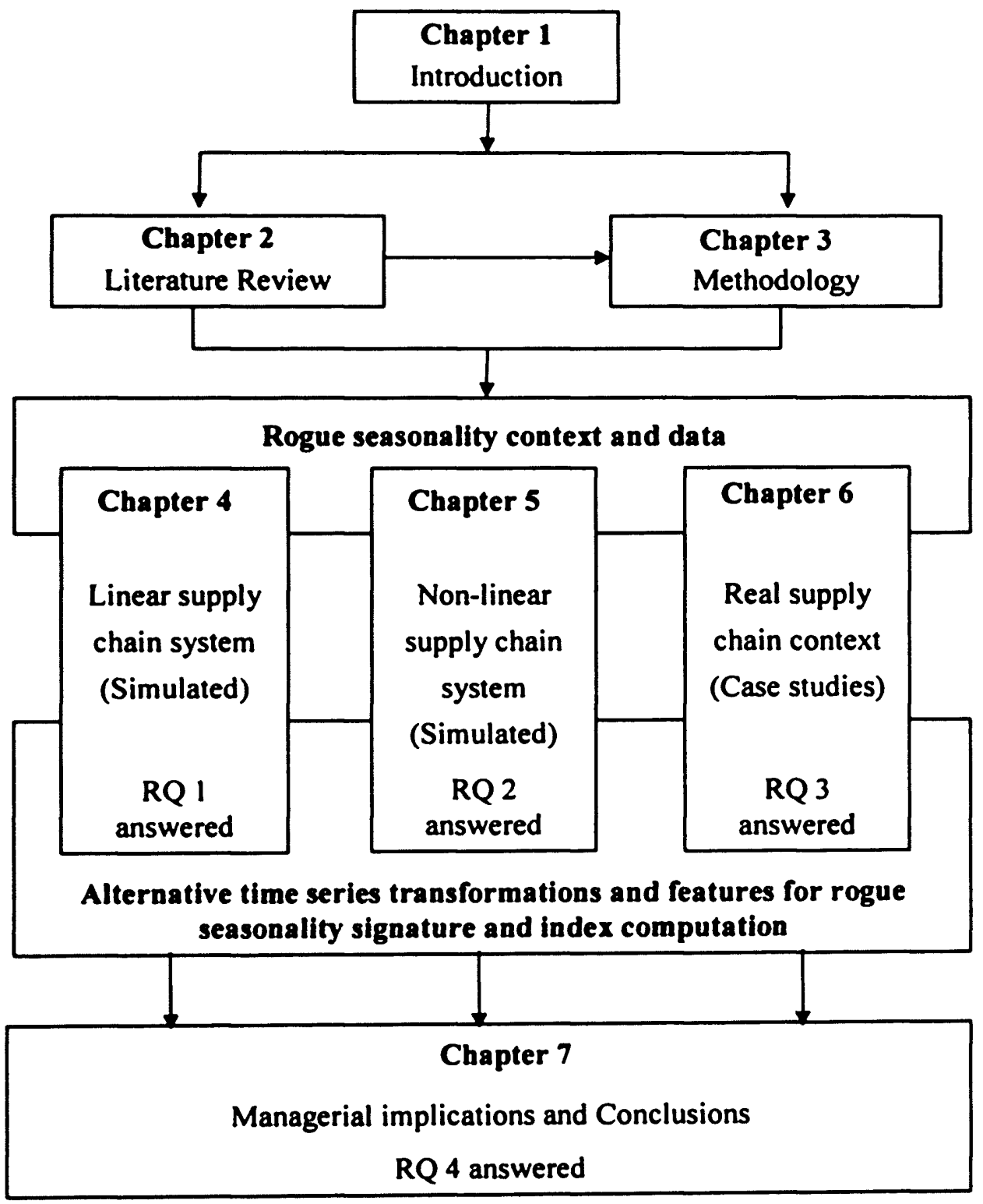

Figure 1.1 Overall Thesis Structure 
Chapter 3 discusses alternative methodologies and the rationale for choosing a multi methodology simulation and case study based approach for understanding rogue seasonality as well as generating related contextual data. Alternative domain representations or time series transformations (features) that could be applied for rogue seasonality detection are also discussed. Some of the work in this chapter is included in the presentation made at the $39^{\text {th }}$ International Conference on Computers \& Industrial Engineering in $\mathbf{2 0 0 9}$ whose full reference is as follows:

Shukla, V. and Naim, M. M. (2009). "The impact of capacity constraints on supply chain dynamics". Proceedings of the $39^{\text {th }}$ International Conference on Computers and Industrial Engineering, July 6-8, Troyes, France

Chapter 4 uses single and three echelon simulated linear supply chain systems to demonstrate the mechanics of rogue seasonality generation. The signature, index of rogue seasonality and the most appropriate time series transformation (and feature) and system variables for computing the same (answer to RQ 1) are also determined from analysis of these systems. This work has been resubmitted to the International Journal of Production Economics and is under review. Its full reference is as follows:

Shukla, V., Naim, M. M. and Thornhill, N. "Rogue seasonality detection in supply chains", International Journal of Production Economics, Under review

Chapter 5 assesses the validity of the findings obtained from the linear supply chain system (signature, index, best time series transformations and features, system variables) on a simulated non-linear supply chain system with backlogs and batching. Use of different number of echelons (four), a different control system and an additional variable for shipment (affects dynamics as well as computation of the index) compared to the linear system provide further robustness to the findings. A part of the work in this chapter has been published in the International Journal of Production Research. Its full reference is as follows:

Shukla, V., Naim, M. M., and Yaseen, E. A. (2009). "Bullwhip and backlash in supply pipelines." International Journal of Production Research, 47(23), pp. 64776497.

Chapter 6 tests the validity of the findings from the simulation analysis on the data from two case studies. Monthly data from a steel case study and daily data from a grocery (soluble coffee) case study are analysed in a similar fashion as chapters 4 and 
5 to answer RQ 3 and in the process support or disprove the simulation findings. The steel case study data analysis is included in the paper resubmitted to the International Journal of Production Economics which is being reviewed (same as in Chapter 4) while the grocery case study findings were presented at the $14^{\text {th }}$ Logistics Research Network Annual Conference in 2009. The full reference for the latter is as follows:

Shukla, V. and Naim, M. M. (2009). "Investigation of disturbances in a grocery supply chain", Proceedings of the $14^{\text {th }}$ LRN Annual Conference, September 9-11, Cardiff, UK.

Finally, Chapter 7 collates the findings from linear and non-linear simulation and the empirical case studies to answer the research questions. Implications of these findings for the managers is also discussed; specifically, the framework which they could use to manage rogue seasonality. This chapter also describes the contributions of the research as well as its limitations and suggests areas of further study.

\subsection{Summary}

This chapter has provided background information on the research theme, the aims of the research and the research questions addressed in this thesis. The structure and contents of the thesis are also explained to give the reader a better understanding of the research process followed. 


\section{Chapter 2 - Literature Review}

This chapter provides an overview of previous research in all areas related to the core theme of the thesis, which is rogue seasonality and its detection. Initially, supply chain management and disturbances in supply chains are discussed, together with the need to focus on and investigate individual disturbances in detail. The next part highlights the significance of control disturbance, its manifestation as the Bullwhip effect and rogue seasonality, and the fact that a large body of research has been conducted on the former while research on the latter has been limited. This leads to the rationale of focussing on the rogue seasonality control disturbance. After discussing the limitations of alternative design based approaches due to system complexity and behavioural dynamics, managing the rogue seasonality by using detection based approaches is explained. Subsequently, the relative merits of the two generic methods of detection: change point detection and signature based methods are presented, together with the suitability of the latter for detection in supply chain contexts. Next, details of the signature based methods which are mostly based on time series clustering and the different time series representations (transformations) and features used in clustering are provided. Finally, all the different threads are drawn together and summarised and from these the research questions are developed.

\subsection{About supply chain management}

Consumers need products and producers produce them. However, difficulties arise when one tries to link the two together. This happens because while consumers are geographically scattered, products are manufactured only at a few centralised locations so as to take advantage of economies of scale. Consumers are also demanding in terms of service and expect instant availability while manufacturing and delivery of products takes time. Finally, consumers are also quite price sensitive and therefore the producer-consumer linkage needs to be efficient as well. These are fundamental issues which are faced by most organisations and give rise to questions such as: What kind of structures should be used to supply products to consumers? When, where, and how much should be produced and supplied? Finally, how best to address these questions while meeting the twin objectives of efficiency and high customer service? 
The study of supply chain management helps answer these questions, where supply chain refers to all entities between producers and consumers (such as distributors, wholesalers, transporters and retailers) which are linked together by the upstream flow of orders (which also constitutes information flow) and downstream flow of material. The information flow is typically used by individual echelons for planning purposes that is distribution and production planning. Traditionally, entities along the supply chain have operated independently with their own, often conflicting, objectives (Ganeshan and Harrison, 1995). However, recently there has been increasing recognition of the benefits from coordinating the actions of these entities. Such an integration strategy is called supply chain management.

Supply chain and supply chain management definitions in the literature recognise the systemic nature of supply chains and the benefits from such an approach. For example, Stevens (1989) defines a supply chain as "a system whose constituent parts include material suppliers, production facilities, distribution services and customers linked together by the feed forward flow of materials and feedback flow of information." According to Christopher (1992), a supply chain is the network of organisations which are involved through upstream and downstream linkages in the different processes and activities, where each produces value in the form of products and services in the hands of the ultimate consumer.

Supply chain management, on the other hand, are those approaches which integrate the constituent parts of organisations in the supply chain. Integration ensures that merchandise is produced and distributed in the right quantities, to the right locations, and at the right time so that both the requirements of providing high customer service and minimum system wide costs are met (Simchi-Levi et al., 2002). A more holistic perspective is used by Chopra and Meindl (2004), who state that supply chain activities begin with a customer order and end when a satisfied customer has paid for its purchase. According to them, the main objective of a supply chain is to match supply with demand while maximising the overall value generated, that is, the difference between product price and cost incurred by the supply chain in fulfilling the customer demand. Matching supply with demand and managing the supply chain as a system is difficult. One of the main reasons for this difficulty is the unpredictable 
disturbances which constantly prevent the supply chain from achieving its performance objectives (Davis, 1993).

\subsection{Disturbances in supply chains}

Supply chains are prone to disturbances. Defined as random or unexpected occurrences that deviate from 'normal' (Svensson, 2000), these could be major but infrequent such as earthquakes and terrorist attacks or minor but frequent such as delays in material deliveries, unanticipated process losses and information interruptions which negatively impact firms across the network. A spate of major and minor disturbances in well known companies has brought this subject into sharper focus in recent times. For example, a ten minute fire at one of its Tier 2 component manufacturers caused Ericsson a financial loss of \$220 million (Norrman and Jansson, 2004) while in another case Cisco had to write off inventory worth $\$ 2.25$ billion because of its dysfunctional planning in a deteriorating economy associated with the "Dot-com" crash (Barrett, 2001). Besides these well known and widely publicised disturbances, there are other routinely occurring disturbances of a lower scale such as delays in material deliveries, unanticipated process losses and information interruptions which cause loss in revenues (lower product availability, loss of customer goodwill) as well as an increase in costs (waste generation and reduction in process efficiencies). Since these disturbances are frequent, supply chain planners tend to internalise them as the "cost of doing business" (Stauffer, 2003). However, over a period of time, the cost implications of such routine disturbances to an organisation are significant. It is to be noted that 'disturbances' have been used almost synonymously with other terms such as 'risks' in the literature. While risks refer to the potential of a negative consequence, disturbances have been used more in terms of the active occurrence of a negative event. For example, in the context of supplier delivery, risk refers to the possibility of material not being delivered on time leading to a loss in production while disturbance refers to the actual realisation of this event. Since their underlying meaning is the same, risk and disturbance have been used interchangeably in the rest of the thesis.

Disturbances have been studied from a multidimensional perspective by different authors. Cavinato (2004) used a holistic perspective by considering disturbances arising in any of the physical, financial, informational, relational, or innovational 
flows in the supply chain. However, a majority of researchers have considered an order fulfilment perspective (physical and information flows), although their classification of disturbances and focus for managing these disturbances are different. An exemplar rather than exhaustive compilation of studies on supply chain disturbances is given in Table 2.1. The key observations from this table are summarised below.

- The negative impact of supply chain disturbances on organisational performance is recognised by almost all studies, though the nature and extent of the impact is anecdotal in many cases. A more quantitative estimate is provided by Hendricks and Singhal (2005) who determined the association between supply chain "glitches" (same as disturbances) and operating performance by analysing the financial statements of the affected companies. They found that firms with "glitches" reported on average $7 \%$ lower sales growth, $11 \%$ higher growth in cost, and $14 \%$ higher growth in inventories compared to a sample of firms of similar size from similar industries without "glitches". The companies with "glitches" also showed a slower economic recovery: operating income, sales, total costs, and inventories did not improve in the two-year time period after the announcement of the "glitch". Similarly, Wagner and Bode (2008) used a cross sectional survey to highlight the negative correlation between supply, demand risks and supply chain performance.

- Most studies are either purely conceptual or empirical but even in the empirical based studies, the research has been conducted at a high level using primarily qualitative approaches (Svensson, 2000; Zsidisin, 2003; Norrman and Jansson, 2004). More quantitative, empirical survey based studies on disturbances by Hendricks and Singhal (2005) and Wagner and Bode (2008) also have a similar macro orientation. Another aspect about the studies on supply chain disturbances is their non consideration of realistic multi echelon structures. This could be because of difficulties both in getting access to such data as well as in analysing it, in particular where there is evidence of 'rippling' of disturbances as they cascade across echelons making the data dynamically complex to scrutinize. This is evident from studies such as Harland et al. (2003) and Hallikas et al. (2002), which are among the few empirical studies about disturbances in multi-echelon supply chains, but who have also used the high level descriptive explanation and analysis approach. 
Table 2.1 Select studies on Supply Chain Disturbances

\begin{tabular}{|c|c|c|c|}
\hline Authors & Disturbance basis used & Research method & Focus of analysis \\
\hline Svensson (2000) & $\begin{array}{l}\text { Supply as source of disturbance } \\
\text { Quantitative / Qualitative and Atomistic (direct) } \\
\text { / Holistic (indirect) nature of disturbances }\end{array}$ & $\begin{array}{l}\text { Empirical (Case study } \\
\text { and survey) }\end{array}$ & $\begin{array}{l}\text { Conceptual framework for supply vulnerability in terms of Quantitative / } \\
\text { Qualitative and Atomistic (direct) / Holistic (Indirect) disturbances }\end{array}$ \\
\hline $\begin{array}{l}\text { Zsidisin et. al. (2000, } \\
\text { 2004); Zsidisin (2003) }\end{array}$ & Supply as source of disturbance & $\begin{array}{l}\text { Empirical (Case } \\
\text { study) }\end{array}$ & Risk identification, assessment and management practices in companies \\
\hline $\begin{array}{l}\text { Giunipero and } \\
\text { Eltantawy (2004) }\end{array}$ & Supply as source of disturbance & Conceptual & Risk management and factors that affect level of investment on the same \\
\hline Blackhurst et al. (2008) & Supply as source of disturbance & $\begin{array}{l}\text { Empirical (Case } \\
\text { study) }\end{array}$ & Index for assessing and monitoring supply risk \\
\hline Johnson (2001) & Supply and Demand as sources of disturbance & $\begin{array}{l}\text { Empirical - } \\
\text { Secondary data }\end{array}$ & Investigation of risk management practice in toy industry \\
\hline $\begin{array}{l}\text { Lindroth and } \\
\text { Norrman* }(2001)\end{array}$ & Supply and Demand as sources of disturbance & $\begin{array}{l}\text { Empirical (Case } \\
\text { study) }\end{array}$ & Identification of risks and risk mitigation by sharing of risks \\
\hline Harland et. al. (2003) & $\begin{array}{l}\text { Supply, Demand and Process as sources of } \\
\text { disturbance in a network environment }\end{array}$ & $\begin{array}{l}\text { Empirical (Case } \\
\text { study) }\end{array}$ & $\begin{array}{l}\text { Risk identification, assessment and management in a network } \\
\text { environment using a proposed tool }\end{array}$ \\
\hline Hallikas et al (2002) & $\begin{array}{l}\text { Supply and Demand as sources of disturbance } \\
\text { in a network environment }\end{array}$ & $\begin{array}{l}\text { Empirical (Case } \\
\text { study) }\end{array}$ & $\begin{array}{l}\text { Identification and assessment of risks in a network environment for the } \\
\text { cases considered. Related conceptual framework and tool proposed. }\end{array}$ \\
\hline $\begin{array}{l}\text { Norrman and Jansson } \\
(2004)\end{array}$ & $\begin{array}{l}\text { Sources of Disturbance - Supply, Demand, } \\
\text { Process and Environmental as sources of } \\
\text { disturbance in a network environment }\end{array}$ & $\begin{array}{l}\text { Empirical (Case } \\
\text { study) }\end{array}$ & $\begin{array}{l}\text { Risk identification, assessment and management processes used in the } \\
\text { case study }\end{array}$ \\
\hline Juttner (2005) & $\begin{array}{l}\text { Environmental, Network and Organizational as } \\
\text { sources of disturbance }\end{array}$ & $\begin{array}{l}\text { Empirical (Focus } \\
\text { group) and } \\
\text { Conceptual }\end{array}$ & Risk identification, assessment and management practices \\
\hline $\begin{array}{l}\text { Kleindorfer and Saad } \\
(2005)\end{array}$ & $\begin{array}{l}\text { Nature of Disturbance - Risks in coordinating } \\
\text { supply and demand, disruptions to normal } \\
\text { activities }\end{array}$ & Conceptual & Risk assessment and mitigation for disruption risk management \\
\hline
\end{tabular}


Table 2.1 Continued

\begin{tabular}{|c|c|c|c|}
\hline Authors & Disturbance basis used & Research method & Focus of analysis \\
\hline Craighead et. al (2007) & $\begin{array}{l}\text { Risks in coordinating supply and demand, } \\
\text { disruptions to normal activities }\end{array}$ & $\begin{array}{l}\text { Empirical (Case } \\
\text { study) and Conceptual }\end{array}$ & $\begin{array}{l}\text { Design characteristics and mitigation capabilities that affect the severity } \\
\text { of disruptions }\end{array}$ \\
\hline $\begin{array}{l}\text { Mason-Jones and } \\
\text { Towill (1998) }\end{array}$ & $\begin{array}{l}\text { Demand, Manufacturing, Supply, Control } \\
\text { systems as sources of disturbance }\end{array}$ & Simulation & Control and demand uncertainty reduction through information sharing \\
\hline $\begin{array}{l}\text { Gaonkar and } \\
\text { Viswanadham (2004) }\end{array}$ & $\begin{array}{l}\text { Planning level - Operational, Tactical, Strategic } \\
\text { Scale of Disturbance - Deviation, Disruption, } \\
\text { Disaster }\end{array}$ & $\begin{array}{l}\text { Conceptual and } \\
\text { Analytical }\end{array}$ & $\begin{array}{l}\text { Conceptual framework for the classification of risks. Risk management } \\
\text { by designing robust supply chains based on prior identification and } \\
\text { assessment of risks }\end{array}$ \\
\hline $\begin{array}{l}\text { Christopher and Lee } \\
\text { (2004) }\end{array}$ & Control system as source of disturbance & Conceptual & Risk management \\
\hline Wilding (1998) & Control systems as source of disturbance & Conceptual & $\begin{array}{l}\text { Endogenous uncertainty generation from inappropriate/non-holistic } \\
\text { decision/supply chain control processes }\end{array}$ \\
\hline Towill (2005) & $\begin{array}{l}\text { Control as source of disturbance (specifically on } \\
\text { Bullwhip) }\end{array}$ & Conceptual & $\begin{array}{l}\text { Possibility of reducing bullwhip by use of appropriate control } \\
\text { algorithms }\end{array}$ \\
\hline Sheffi (2001) & Environment as the source of disturbance & Conceptual & Risk management \\
\hline $\begin{array}{l}\text { Chopra and Sodhi** } \\
(2004)\end{array}$ & $\begin{array}{l}\text { Supply, Demand, Process and Environmental as } \\
\text { sources of disturbance }\end{array}$ & Conceptual & Optimal and holistic risk management \\
\hline $\begin{array}{l}\text { Hendricks and Singhal } \\
(2005)\end{array}$ & $\begin{array}{l}\text { Mismatch of demand with supply (supply chain } \\
\text { glitch); Supply, demand and process as major } \\
\text { sources of glitches }\end{array}$ & Empirical (survey) & $\begin{array}{l}\text { Extent of adverse impact of supply chain glitches on operating } \\
\text { performance (lower sales growth and higher costs, assets and } \\
\text { inventories) }\end{array}$ \\
\hline $\begin{array}{l}\text { Wagner and Bode } \\
(2008)\end{array}$ & $\begin{array}{l}\text { Supply, Demand, Regulatory, legal and } \\
\text { bureaucratic, infrastructure and catastrophic }\end{array}$ & Empirical (survey) & $\begin{array}{l}\text { Operationalisation of the supply chain risk construct and relationship } \\
\text { between supply chain risks and supply chain performance }\end{array}$ \\
\hline $\begin{array}{l}\text { Tang and Tomlin } \\
\text { (2008) }\end{array}$ & $\begin{array}{l}\text { Supply, Demand, Process and Environmental as } \\
\text { sources of disturbance }\end{array}$ & Analytical & $\begin{array}{l}\text { Risk management by deploying demand, supply and process } \\
\text { flexibilities }\end{array}$ \\
\hline Wilson (2007) & Transport as source of disturbance & $\begin{array}{l}\text { Simulation (System } \\
\text { Dynamics) }\end{array}$ & $\begin{array}{l}\text { Impact on supply chain performance from transport disruption in } \\
\text { different echelons }\end{array}$ \\
\hline
\end{tabular}

Business risks (Financial, Business, Regulatory, Legal) and product design related risks were not included as the focus is on the product delivery process.

* Proposed a framework for positioning supply chain risk issues in terms of unit of analysis - type of risk (in terms of scale) - risk handling focus; ${ }^{* *}$ Exact risk nomenclature used is different

u 
- Most studies have categorized disturbances on the basis of their source or origin, though the sources used differ across studies. Studies by Svensson (2000), Zsidisin (2003), Zsidisin et al. (2000, 2004), Giunipero and Eltantawy (2004) and Blackhurst et al. (2008) focus on disturbances originating at the supplier such as machine breakdowns, capacity constraints and quality problems. Others such as Johnson (2001) and Hallikas et al. (2002) have added demand related disturbances with Davis (1993) and Harland et al. (2003) further adding process related disturbances. Demand disturbance refers to variations in nature and volume of customer demand while process disturbance refers to disturbances such as those mentioned above for supplier disturbance which occur in an echelon's own value adding process. Inappropriate information and control systems for matching supply and demand and its associated implications is recognised as a control disturbance by Mason-Jones and Towill (1998) and Christopher and Lee (2004). However, few studies have recognised it as a separate disturbance with most including it within demand risk. Finally, disruptive events such as earthquakes, terrorist incidents, fires and strikes have been classified as a separate environmental disturbance or disruption by Norrman and Jansson (2004), Juttner (2005) and Kleindorfer and Saad (2005).

- In terms of managing risks or disturbances, the Association of Insurance and Risk Managers (AIRMIC, 2002) prescribes four generic steps, which adapted for supply chain risks by Norrman and Johnson (2004) are: Risk identification (Identification of direct and indirect potential sources that could negatively affect the supply chain), Risk assessment (Prioritization of the risks identified based on their probability of occurrence and consequences), Risk management (Implementation of actions such as accepting, avoiding, reducing, sharing or transferring risks to reduce its consequences or probability of occurrence or both), Risk monitoring (Monitoring of large unresolved risks). Empirical evidence from the studies suggests that very few organizations follow these four steps rigorously. A study by the Computer Sciences Corporation had a similar observation: $43 \%$ of 142 companies studied reported their supply chains to be vulnerable to disruptions out of which $55 \%$ did not have any documented contingency plans (Poirier and Quinn, 2003). One reason for this could be the scarcity of suitable managerial techniques as highlighted by Christopher and Lee (2004) and Blackhurst et al. (2008). 
Disturbances impact organisational performance negatively. The movement towards increased responsiveness, higher levels of agility and lower inventory levels has further increased the potential occurrence of such disturbances and resulting loss to organisations (Christopher, 2002). The subject of supply chain risk management is therefore important and requires intensive study. However, the concept of risk in supply chains is still in its infancy (Juttner et al., 2003). Most studies on the subject are at a 'high' conceptual level which provide a 'big picture' understanding of the risks and their management, but lack details. Researchers are unable to 'drill down' to the key variables influenced by or influencing the disturbances, the relationships among them and methodologies or tools to manage them. These studies also have lower practical utility as real applications require dealing with detailed decision rules, controls, procedures and circumstances. This has been highlighted by Blackhurst et al. (2005) who comment that from a practical perspective high level studies provide limited insights into how to deal with disturbances. Therefore, instead of the generic 'big picture' approach to study disturbances, there is a need to focus on individual disturbances and investigate them in detail. Such an approach was used by Svensson (2000) and Blackhurst et al. (2008) for supply and by Wilson (2007) for transport disturbance, although the detailed nature of investigation as used in the thesis is much more comprehensive.

This thesis focuses on control disturbance. Control disturbance is endogenously generated from use of inappropriate information and/or control algorithms to match supply with demand (Mason-Jones and Towill, 1998; Geary et al., 2002). It has severe negative cost implications for firms and therefore its study is important. For example, Metters (1997) showed an individual firm incurring $10 \%$ to $30 \%$ additional costs from control disturbances. Propagation of these disturbances causes the cost implication to be even more severe in a multi echelon supply chain. Moreover, because of the casual attitudes of practitioners who either neglect them, or ignore them or passively accept them (McCullen and Towill, 2002) these disturbances continue to affect many supply chains. Though a few researchers such as Wilding (1998), Christopher and Lee (2004) and Towill (2005) have studied these disturbances, their approach was primarily conceptual while in this thesis a detailed data based approach is used. Moreover, the analysis in the thesis is based on multi echelon supply chains, and therefore 
contributes both to the limited literature on analysis of such structures and also increases the external validity of the findings.

\subsection{Supply chain control disturbance}

Each echelon in a multi echelon supply chain is required to solve a fundamental problem, which is: "how much to order from its upstream echelon which satisfies customer demand but also keeps its inventory level low". This is the supply chain control problem. It is analogous to the production/inventory control problem whose purpose is to transform incomplete information about the market place into coordinated plans for production and replenishment of raw materials (Axsater, 1985). The task is difficult as both market place demand and receipts from suppliers is uncertain and generally involves the use of information such as demand, inventory and orders in the pipeline in the decision making, which are applied in a mathematical way using replenishment rules or on the basis of judgement. For a multi echelon supply chain the difficulties are manifold. This is because of the dynamic complexity from the interactions of individual echelons with different ordering practices and the generation of endogenous disturbances called control disturbances (Mason-Jones and Towill, 1998). These disturbances have been characterised by Geary et al. (2002) as arising from unavailability, non timeliness and inaccuracy of information and/or use of inappropriate control systems which use this information to determine procurement, supply and manufacturing orders. Typical control disturbances are the Bullwhip effect (Lee et al., 1997a, b) in which the order variability is increasingly amplified from downstream to upstream echelons and rogue or endogenously generated seasonality (McCullen and Towill, 2002) in which orders and other supply chain variables oscillate when no oscillation is present in the exogenous demand or oscillate at a frequency different from exogenous demand. Both these effects were demonstrated by Forrester (1961) using a simulation experiment. However, since then, while there has been extensive research on the Bullwhip effect (Geary et al., 2006), research on rogue seasonality has been minimal (Kim and Springer, 2008).

\subsubsection{The Bullwhip effect}

The Bullwhip effect has been observed in various empirical sectors such as apparel, 
grocery, food, automotive, electronics and retail and across a number of echelons (Miragliotta, 2006). This effect has also been studied extensively using various methods (Geary et al., 2006; Miragliotta, 2006; Disney and Lambrecht, 2007). Different causes have been identified for its generation with researchers typically focussing on a single cause in their respective study.

\subsubsection{Causes of the Bullwhip effect}

The Bullwhip effect is associated with the upstream amplification of orders and Lee et al (1997a, b) identified and analysed four rational causes for its occurrence. Besides these rational causes, two additional causes from a behavioural perspective (bounded rationality and related biases in decision making) have been proposed by Sterman (1989) and Croson et al. (2005).

a) Demand signal processing and lead times (Forrester effect) - The Bullwhip effect arises from the compounding of forecasts due to use of downstream orders rather than end customer demand in forecasting. Delays in information transmission and shipment further accentuate the order amplification as longer delays mean more safety stocks and safety stocks are typically added to forecasted demand for determining order quantity (Axsater, 2000). Studies have varied the nature of demand, forecasting process, inventory replenishment rules and tried information sharing to determine its impact on the Bullwhip effect (Miragliotta, 2006).

b) Order batching - Aggregation of orders in a supply chain is the cause of order amplification in this case. Reasons for aggregation or batching could be economies of scale in production or transport (Burbidge, 1989), use of manufacturing resource planning (MRP) systems, the "hockey stick" phenomenon when multiple companies order at the same time (Lee et al, 1997a, b), economic order quantity (EOQ) based ordering and economic production quantity (EPQ) based production where set up times/costs are high (Hejazi and Hilmola, 2006). Analytical and simulation studies on this cause of Bullwhip have found a positive correlation between batch size and order amplification (Kelle and Milne, 1999; Holland and Sodhi, 2004; Potter and Disney, 2006; Cachon, 1999; Riddalls and Bennett, 2001).

c) Rationing and Gaming - First highlighted by Houlihan (1987), this refers to the tendency of customers to over order during perceived threats of shortages or delivery 
unreliability. For example, a perception of production capacity shortage could prompt customers to inflate their orders, so that even after rationing they could still receive their desired requirement (Lee et al., 1997a, b) or to place multiple orders with different suppliers, which could be subsequently cancelled when the perception/actual shortage situation improved. Both these situations create demand distortions and amplifications. Cisco's inventory write off problem (Barrett, 2001) was primarily on account of this cause. Few studies have addressed this cause of Bullwhip and those which have, have studied it from a contracting perspective using analytical methods (Cachon and Lariviere, 1999a, b, c).

d) Price fluctuation or the Promotion effect - This is related to the practice of using promotions to stimulate demand. Demand increases in the short term, as customers take advantage of this opportunity to forward buy but the resulting excess stock causes demand in subsequent periods to fall. This distortion in demand has a significant adverse impact on the supply chain dynamics. This cause of Bullwhip has also seen limited research with one of the few studies on this subject being by Lummus et al. (2003).

e) Behavioural causes - Two additional causes of Bullwhip have been proposed which are attributed to non rationality in decision making and have been identified on the basis of experimentation with human subjects in a role playing game, the Beer game (Sterman, 1989). The supply line underweighting (SLU) cause is characterised by participants underestimating the supply line (orders placed but not delivered) and placing excess orders resulting in the Bullwhip effect (Sterman, 1989). A different behavioural bias called coordination stock was identified by Croson et al. (2005) who observed variability in orders despite the demand being constant and which was known to all the players. According to the authors this was because individual players ordered more so as to avoid the risk of poor decisions made by other players.

\subsubsection{Methods used to study the Bullwhip effect}

Researchers have used various methods to study this subject such as statistical or operational research (OR) methods, control theory methods, system dynamics simulation and management games. 
a) Statistical/OR methods - This approach involves the formulation of the problem as a difference equation under assumed operating conditions (demand, lead time, forecasting and ordering processes) which is explicitly solved by minimising a cost function (or surrogate). The dynamic performance such as order amplification is implied by the mathematical solution. This method has mostly been used to study the Forrester effect under different settings. For example, Lee et al. (1997b) and Chen et al. (2000) investigated the impact of stationary AR (1) demand, different forecasting techniques and an adaptive base stock policy while Daganzo (2004) focused on the relative value of past demand information on the Bullwhip effect.

b) Control theory methods - This method involves representing the supply chain (structure as well as forecasting and ordering policies) in an input-output format which is then converted into a transfer function and analysed to draw conclusions about its impact on the Bullwhip effect. The impact of different demand processes, forecasting techniques, and ordering policies have been investigated using this approach, a summary of which is provided in the review papers by Ortega and Lin (2004) and Sarimveisa et al. (2008).

c) Systems dynamics simulation - This method was advocated by Forrester (1961) as a method of investigating the dynamical effects in large, complex systems. This method's usage involves representing the relationships between variables as causal loop diagrams, converting them into differential equations and then visually studying the output profiles of variables to understand the cause effect relationships. This approach has significant advantages vis-à-vis analytical studies in terms of ability to handle multi echelon structures, arbitrary demand process, forecasting and ordering policies as well as non-linearities such as backlogs and capacity constraints. It has therefore been extensively applied for understanding supply chain dynamics especially the Bullwhip effect in multiple settings. For example, Potter and Lalwani (2008) use system dynamics simulation to investigate the impact of demand amplification on freight transport while Wilson (2007) uses it to assess the impact of transport disruption on supply chain performance. A review of studies based on system dynamics modeling in supply chain management is given in Angerhofer and Angelides (2000). 
d) Management games - Management games, especially the Beer game (Sterman, 1989) has been used to understand the Bullwhip effect. This game represents a typical production-distribution system with four echelons (retailer, wholesaler, distributor and factory) where the player managing each echelon enacts the role of an inventory manager and makes decisions on ordering from his/her supplier. The decision environment is complex with a non-stationary demand pattern, time delays in ordering and shipment, uncertainty in behaviour of suppliers and customers, short response times and the need to optimise inventory/backlogs. Despite being a considerable simplification of reality, the Beer game structure is still representative of many real world supply chains and it is therefore not surprising to see the game being used in many Bullwhip related studies (Croson and Donohue 2003, 2006, Disney et al. 2004, Diana and Katok 2006). Though the focus of these studies has been on the behavioural origins of the Bullwhip effect and for which the role playing version of the game was used, the Beer game has also been simulated in many studies (van Ackere et al., 1993; De Souza et al., 2000; Shukla et al., 2009). Beer game incorporates non-linearities from backlogs, multiple echelons, time delays as well as the shipment variable which make it realistic and therefore more attractive for simulation.

\subsubsection{Measurement of the Bullwhip effect}

Multiple measures of Bullwhip have been proposed in the literature, which originate from the different methods used to study it. Studies such as Lee et al. (1997b) and Chen et al. (2000), which use statistical methods, consider the ratio between order and demand variances as the measure, with a ratio greater than one indicating Bullwhip. Disney and Towill (2003) however, argue that this measure reflects only half of the Bullwhip problem and suggest inclusion of the ratio between net stock and demand variance in the measurement. Use of the coefficient of variation rather than variance has been suggested by Fransoo and Wouters (2000) as it eliminates mean differences between the profiles while Taylor (1999) has proposed standard deviation in place of variance. Taylor (1999) also demonstrated the intra organisational existence of Bullwhip, unlike most studies which focus only on Bullwhip across organisations, and highlighted the need to measure it using activity level information. 
System dynamics based studies typically use a step input as the test demand pattern, and hence peak order value is used as the measure of order amplification and Bullwhip. This is used as the Bullwhip measure in Beer game studies also, which use step as the exogenous demand profile. Researchers using the filter theory approach such as Dejonckheere et al. (2003) have defined "noise bandwidth" as the Bullwhip measure which is the area under the squared frequency response (FR) curve. What is the best measure among the above? Towill et al. (2007) tried different measures: peak order, variance and noise bandwidth with a simulated data set and found Bullwhip assessment to be conflicting. While no Bullwhip was indicated by the variance measure, the peak order and noise bandwidth measures indicated significant Bullwhip. They rightly caution that "Bullwhip is in reality not a generic term meaning the same thing to all system users. Instead it is application specific". In terms of the Bullwhip measure to be used they state that "which Bullwhip measure to use should not depend on the mathematics/simulation tools exploited in scheduling packages, but on the user operating scenario". Hence, the appropriate measure to be used is entirely contextual and based on requirements.

Overall it was found that research on control disturbance related to the Bullwhip effect has been extensive. It has been studied empirically, its causes have been identified by using multiple methods, various measures have been proposed for its measurement and ways to mitigate it have been suggested. Also, most cause and mitigation oriented studies have focussed on design aspects such as design of structure and information flows (number of echelons, information sharing, lead times) and design of control practices (forecasting and ordering), the assumption being that Bullwhip can be designed away. The other control disturbance besides the Bullwhip effect is rogue seasonality which is discussed below.

\subsubsection{Rogue seasonality}

Rogue seasonality refers to cyclic patterns in orders and other supply chain variables, which are endogenously generated from the inventory and production control systems used, and are not present in exogenous customer demand. In the case of cyclicality being present in exogenous demand, rogue seasonality manifests itself as orders and other supply chain variables oscillating at a different frequency than exogenous demand. Rogue seasonality could wrong foot the decision makers who, believing 
rogue seasonality to be real demand, could either be unnecessarily ramping their production up or down and increasing their on-costs or buffering themselves via use of inventory and increasing their holding costs. Moreover, rogue seasonality may not be localised but may propagate due to the Forrester effect, causing cost inefficiencies throughout the supply network. Metters (1997) showed that costs to the extent of 10$20 \%$ could be reduced by elimination of such seasonal variations.

Rogue seasonality is present in many supply chains and to highlight this, sample data available in the literature is used as given in Figure 2.1. The data was used in the context of Bullwhip in the respective studies.

The first plot in Figure 2.1 depicts the dynamics in a three echelon electronic supply chain (Kaipia et al., 2006). Rogue seasonality is evident with the oscillation frequency of the upstream variable (Supplier shipments to OEM) different from customer sales (channel sell-through). The second plot (Lee et al., 1997a) shows the orders placed not only amplified in relation to actual sales, but also oscillating with almost constant periodicty, though no such periodicity is apparent in the actual sales profile. Finally, the third plot is the ouput generated from the empirical simulation of a four echelon beverage supply chain which also shows periodic oscillations in the most upstream variable (procurement) whose periodicity is different from customer sales (Torres and Moran, 2006).

\subsubsection{Previous research on rogue seasonality}

Few studies have looked at rogue seasonality specifically with most studies considering it together with Bullwhip. This is also evident in the recent review of Bullwhip effect by Miragliotta (2006). It was first demonstrated by Forrester (1961) who used a three echelon factory-distributor-retailer simulation model to demonstrate the Bullwhip effect as well as the generation of seasonality (periodicity of 5-10 months) from random customer demand. He highlighted the role of system characteristics such as its structure, feedback loops, time delays and ordering policies for this generation. McCullen and Towill (2002) also used a similar approach to demonstrate and explain both Bullwhip and rogue seasonality. Oscillation in orders and inventory was also observed in a role playing four echelon supply chain game (Beer game) which was subjected to a step change in demand (Sterman, 1989). 

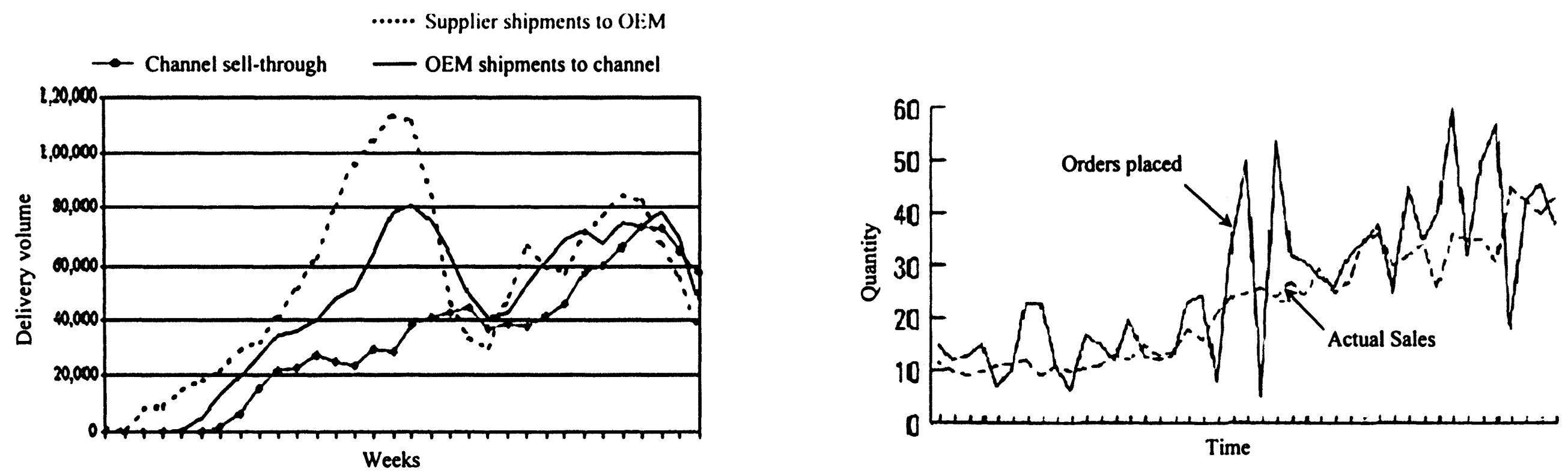

(Source: Kaipia et al., 2006)

(Source: Lee et al., 1997a)

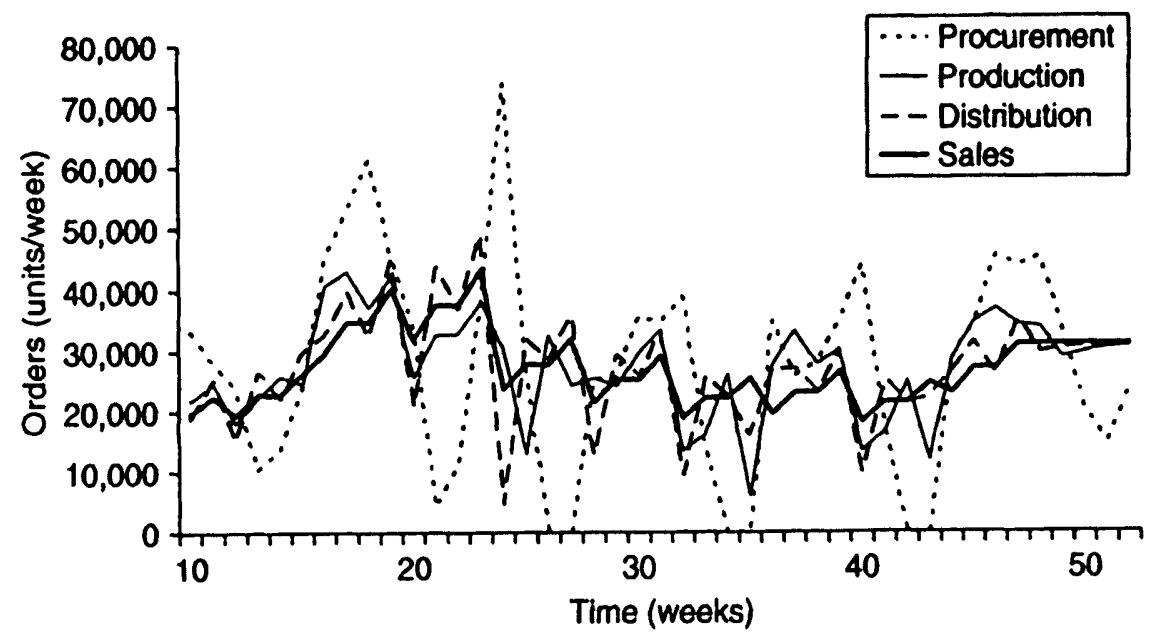

(Source: Torres and Moran, 2006)

Figure 2.1. Rogue seasonality generation in different empirical contexts 
Besides the reasons suggested by Forrester, an additional factor suggested for this behaviour was the the inability of the players to account for the supply line (SLU).

Some other studies such as those by Towill (1982), Towill and del Vecchio (1994), Towill et al. (2007), Dejonckheere et al. (2003) and Jaksic and Rusjan (2008) have indirectly studied amplification of rogue frequencies in view of their use of control theory, specifically frequency response (FR) analysis to study the Bullwhip effect. These studies have exploited the mathematical equivalence between time and frequency domain representations to analyse the order amplification in the latter domain with Dejonckheere et al. (2003) even deriving a measure of Bullwhip effect in this domain called "noise bandwidth" (area under the squared frequency response of the order variable). Jaksic and Rusjan (2008) and Dejonckheere et al. (2003) also studied the impact of different forecasting and replenishment policies on order amplification using the frequency domain approach. However, it is to be noted that in these Bullwhip oriented studies, frequency domain analysis is done for only one (order) variable with information on all frequency channels being used. Though rogue seasonality is also analysed in the frequency domain which is suited for data with cyclicality, (Chatfield, 2004), the nature of analysis is different as will be seen in later chapters.

The literature review identified only two studies which were exclusively focussed on rogue seasonality. Kim and Springer (2008) used an analytical system dynamics approach with a dyadic structure to determine the conditions under which rogue seasonality could arise in a supply chain. The study assessed the role of ordering policy parameters, that is, the weights assigned to inventory gap and pipeline gap terms in generating strong and weak cyclicality, where strong cyclicality was defined for the behaviour where variables (specifically inventory and pipeline stock) alternately overshot and undershot their equilibrium values before converging while cyclicality was defined to be weak if they did so only once. Though focussed on rogue seasonality, the study by Kim and Springer is not comprehensive; it considers only a dyadic structure, it does not consider the impact of forecasting policies on the dynamics and analyses the dynamics of only two system variables (inventory and pipeline stock). Secondly, the study defines rogue seasonality from a subjective perspective, though with a frequency bias; a profile with multiple oscillations is 
classified as strong cyclicality while that with one or less cycle as weak cyclicality. On the other hand, studies such as Metters (1997) consider amplitude of seasonality to be a more relevant factor in terms of adverse impact on cost efficiency of operations. Finally, Kim and Springer complicate their classification of rogue seasonality further, by introducing another factor called external shock whose magnitude (small/large) also determines the nature of rogue seasonality (strong / weak).

The second study on rogue seasonality is empirically oriented and uses the context of a steel supply network (Thornhill and Naim, 2006). Multiple variables are analysed using a specific technique to characterise the cyclical disturbances in the network. The technique called spectra principal component analysis (SPCA) involves the use of spectra (frequency domain representation) to make variable profiles invariant to lags, PCA (Principal Component Analysis) to reduce dimensionality by exploiting the correlation between the spectra and plotting and clustering the spectra to discriminate rogue seasonality from seasonality in exogenous demand. However, this exploratory study used only a single technique for rogue seasonality detection and tested it using only one case study. The technique used was also not automatic and required many decisions to be made in terms of choosing the number of principal components (which also needed to be less than three to enable plotting), deciding cluster membership from the plots and interpreting profiles of the clusters. Findings from this study by Thornhill and Naim need to be validated and extended through multiple rogue seasonality contexts, alternative techniques for detection, and use of a more automated process.

It is evident that rogue seasonality is present in many supply chains and cause significant cost inefficiency which makes its study quite important. However, the research focus on it has been quite limited, with most studies continuing to treat it as a part of the Bullwhip effect. Thus far no measure for rogue seasonality has been defined. Also, an exhaustive search of the literature revealed just two studies with an exclusive focus on rogue seasonality. The first study had a limited scope and was focussed on identifying ordering control parameters to reduce rogue seasonality generation and is therefore similar to the vast majority of similar Bullwhip effect studies, which seek to design out control disturbances by appropriate choice of policies and parameters. The second study investigated rogue seasonality in an 
empirical setting but limited themselves to one supply network and one technique, with the technique used also requiring manual inputs during application.

A more rigorous and intensive study of the rogue seasonality in supply chains is therefore required similar to that done for the Bullwhip effect (see Section 2.3.1). One way to proceed would be to follow the Bullwhip research pathway and design control policies which eliminate or minimise the generation of rogue seasonality.

\subsubsection{Issues with design based approaches to manage control disturbances}

A vast majority of studies on control disturbances (mostly Bullwhip effect oriented and one on rogue seasonality) have focussed on determining appropriate control parameters so that such disturbances could be a-priori designed out of the system. Though no doubt important, such an approach may be less effective in many real world environments. This is because real decision making contexts are in general complex, and therefore, less amenable to control. More importantly, the prescriptions for controlling such complex contexts have mostly been derived using simplistic dyadic structures and which unrealistically assume complete rationality in decision making.

\subsubsection{Complexity in supply chains}

Real world supply chains are complex. The major factors which make supply chains to be complex are:

- Multiple information and material flows - A complex web of interlinked information and material flows spanning multiple suppliers, manufacturers and distributors (Lee and Billington, 1993) which have arisen from market pressures to drive down costs, introduce new products and expand into new markets.

- Dynamic nature and uncertainty - This arises from the state of flux and transient state that supply chains continuously operate in (Haywood and Peck, 2004) from changes such as in marketplace demands and product specifications, uncertainties such as in capacity, availability, manufacturing and transportation times and quality and continuous improvement initiatives within the organizations and the wider 
industry as a whole. The interactions between the supply chain partners and propagation of uncertainties further complicate the dynamics (Panunak, 1999).

- Conflicting objectives among partners - Difference in the constraints and objectives of individual companies cause multiple rather than a single, unified plan for the supply chain (Ganeshan and Harrison, 1995; Hausman, 2003). Even if a few partners in a supply network have conflicting objectives, such as maximisation of their individual profits, this could disturb the dynamics across the network both in terms of information/material flows as well as profitability.

The resulting complexity from dynamic interactions, among multiple echelons with different objectives, in an environment which is uncertain, makes design based control difficult. This has been recognised by many researchers such as Baader and Montanus (2008) who comment that "Because of the growing complexity of logistical structures, the number of planning parameters has expanded so dramatically, that an all encompassing optimisation is not possible. Planning proceeds sequentially, in which actions of previous steps are assumed as constant for optimisation of parameters at the next step. Hence, in planning one generally restricts the focus to a selection of the most important planning parameters. The lofty goals of avoiding "events" can therefore not be realised." Similarly, McCarthy and Tan (2000), Radjou (2002) and Lawrie (2003) also agree with the viewpoint that since supply chains/networks often display unpredictable behaviour, they can never be completely controlled through topdown planning, however collaborative it might be.

Another related factor is that while the complexity of supply chains has increased significantly, the design prescriptions to manage this complexity have not kept pace. Most studies on Bullwhip (covered in Section 2.3.1) and the study by Kim and Springer (2008) on rogue seasonality, which have proposed control design policies and parameters to minimise the magnitude of such disturbances, have done so using dyadic, and therefore, analytically tractable structures. These policies and parameters therefore have limited applicability in real world applications. Another reason for the limited applicability is that these policies and parameters have been derived on the basis of complete rationality in decision making, while departures from rationality are well known in most real world environments. 


\subsubsection{Irrationality in decision making}

Most theoretical work in operations management including control design is based on a normative or prescriptive approach and assumes that decision makers are rational and perfect optimisers. However, detailed tracking of individuals' decisions shows that their observed behaviour often does not fit the predictions of such theoretical models. Rather than conforming to traditional assumptions of narrow self interest and unbounded rationality, people exhibit decision making biases, use heuristics, and prefer outcomes that are not strictly value maximising. The Beer game, despite being a much simpler decision making environment compared to the real world, clearly highlights this divergence between the theoretical and practical. In the game, players take up individual roles (retailer, wholesaler, distributor and factory) in a serial, decentralised supply chain, with each player needing to make just one decision every period: how much to order from its supplier which fills downstream customers demand but does not create either surplus inventory or excess backlogs. Players are found to be unable to apply appropriate ordering policies even in this relatively simple situation. Many players in the game do not adequately account for the supply line (orders placed but not yet received) in their ordering rule and cause the generation of both Bullwhip and rogue seasonality across the supply chain (Sterman, 1989). Even with a simplified decision context with a less complex demand pattern and information sharing between the players, inappropriate ordering behaviour is still observed. For example, Croson and Donohue (2006) used uniform demand instead of step demand used by Sterman and ensured sharing of individual inventory level information across the supply chain but still noted the persistence of the supply line underweighting behaviour in ordering. Similarly, Wu and Katok (2006) used uniform exogenous demand, made supply line visible to the respective players and even repeated the games so that players could benefit from the learning in the previous game but still observed the same underweighting bias in ordering behaviour. Even a constant demand and which was known to all players in the study by Croson et al. (2005) generated Bullwhip effect and rogue seasonality though a rational response in such a situation would have generated a flat order profile at the level of exogenous demand. Communication of the optimal ordering rule to the players, in a subsequent phase of the experiment, also did not improve the ordering behaviour. 
Experiments other than the Beer game have also noted the divergence from predictions of rational models. For example, Schweitzer and Cachon (2000), in their investigation of newsvendor decisions, which are fundamental models of supply chain systems, demonstrated that choices made by human subjects in the experiment systematically deviated from those that maximized expected profit. Subjects consistently ordered amounts lower than the expected profit-maximizing quantity for high-profit products and higher than the expected profit-maximizing quantity for lowprofit products so as to reduce ex-post inventory error, the absolute deviation between the amount ordered and realized demand. Similarly, Loch and Wu (2007) in their review of behavioural operations management refer to a study by Rapoport (1966, 1967) who found that decision makers in a stochastic multistage inventory task generally under-controlled the system, and orders were correlated with past demand even when demand draws were independent. The behavioural bias in decision making is also evident in information technology (IT) based decision support systems (DSS), where decision makers, when swamped with significant amounts of information and complexity resort to use of heuristics or simple rules of thumb (Bendoly and Speier, 2008). Even in an empirical enterprise resource planning (ERP) context, which typically imposes strong and difficult to change structures, Bendoly and Cotteleer (2008) observed systematic circumvention of protocols.

In summary, rationality in decision making has been contradicted in a range of studies. This is on account of the numerous cognitive, informational, temporal and other limitations which bind human rationality (Loch and Wu, 2007; Bendoly et. al, 2006). In the context of real world supply chains, with all their complexities in material and information flows, most of the above highlighted limitations would be operative, causing decision making to be not entirely rational. Normative control policies and parameters, which are based on rationality in decision making, would therefore be less effective in designing away control disturbances in such environments.

Hence to summarise, supply chains are complex entities and therefore difficult to control. Moreover, the policies and parameters used for control are too simplistic in relation to reality (and hence less effective), having being developed under dyadic settings. Finally, there is additional uncertainty about the way these policies and 
parameters are applied in practice, due to the behavioural biases in decision making. Hence, a top-down design based approach for managing control disturbances has significant limitations. Though important and relevant, it needs to be complemented with a bottom-up sense and respond based approach. Rather than uniformly applying generic control design/parameter prescriptions on all supply chains, the more problematic ones could be identified (sensed) to which appropriately contextualised generic rules are applied (respond). This is akin to the management by exception philosophy.

\subsection{Sense or detect and respond based approach}

The sense and respond (SR) based approach was conceptualised by Haeckel (1999) in a strategic context, who recognised the difficulty in planning in an environment where unpredictability was the norm. He drew inspiration from Peter Drucker who wrote that "uncertainty in the economy, society, politics has become so great so as to render futile, if not counterproductive, the kind of planning most companies still practice: forecasting based on probabilities". The SR approach uses a four step decision making cycle called SIDA (Sense-Interpret-Decide-Act) as depicted in Figure 2.2 below. It is adapted from the OODA loop cycle (an acronym for "observe, orient, decide and act) used by Col. John Boyd (USAF) to explain the significant successes of U.S. fighter pilots over their North Korean counterparts during the Korean War. The OODA loop essentially states that if one makes informed decisions and takes action faster than one's enemy, then one will outperform the enemy. As the speed of the OODA cycle increases, the enemy remains continually (both tactically and intellectually), a step behind.
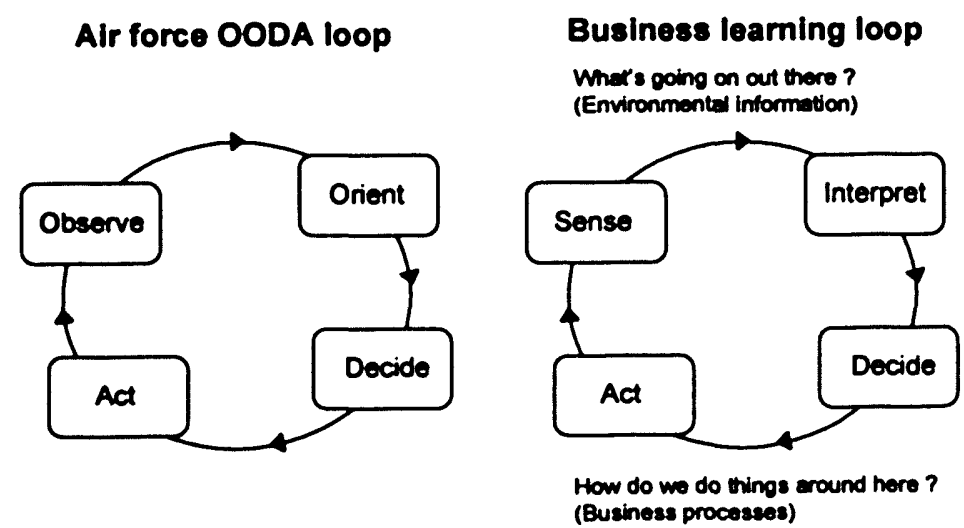

Figure 2.2 Decision making loops related to sense and respond approach

(Haeckel, 1993) 
The components of SIDA are:

- Sense: Refers to the use of probes or data to assess changes in the context

- Interpret: Involves applying context to the data, that is, transforming it into information by using methods such as filters, models and data mining

- Decide: Refers to choosing a particular option from an alternative set of options based on the interpretation of the situation

- Act: Refers to implementing or acting on the choices made

Use of information is central to SIDA with Haeckel (1993) using the term "manage by wire" for businesses which is analogous to modern aviation's fly-by-wire systems. The SR framework has been used in many studies at the strategic level including a recent one by Mathiassen and Vainio (2007) who used it to investigate the dynamic capabilities in two small software companies. The concept of sense or detect (the terms sense and detect have been used interchangeably hereon) has also been applied at an operational level and across multiple domains. This is summarised in Table 2.2.

Table 2.2 Application of detection based approach in different domains

\begin{tabular}{|c|c|c|c|}
\hline Domain & Typical fiformation used & \begin{tabular}{|l|} 
Nature of \\
Detection
\end{tabular} & Aüthors \\
\hline Finance & $\begin{array}{l}\text { Spend pattern - nature, amount, } \\
\text { location, frequency }\end{array}$ & Consumer fraud & Edge and Sampai (2009) \\
\hline Telecom & $\begin{array}{l}\text { Geographical origin and destination of } \\
\text { call, call time, duration and day of the } \\
\text { week when call made }\end{array}$ & Consumer fraud & $\begin{array}{l}\text { Phua et al. (2005) } \\
\text { Fawcett and Provost (1997) } \\
\text { Cortes and Pregibon (2001) }\end{array}$ \\
\hline Stock Trading & $\begin{array}{l}\text { Options and stock related information } \\
\text { such as volumes, different kinds of } \\
\text { prices and market news }\end{array}$ & $\begin{array}{l}\text { Insider trading in } \\
\text { stocks }\end{array}$ & Donoho (2004) \\
\hline $\begin{array}{l}\text { Equipment } \\
\text { Maintenance }\end{array}$ & $\begin{array}{l}\text { Operating parameters, vibration } \\
\text { characteristics, cutting fluid chemistry }\end{array}$ & $\begin{array}{l}\text { Condition } \\
\text { monitoring/ fault } \\
\text { detection of } \\
\text { equipment }\end{array}$ & $\begin{array}{l}\text { Raheja et al (2006) } \\
\text { Lee et al. (2006) } \\
\text { Olsson et al. (2004) } \\
\text { Jeong et al. (2006) }\end{array}$ \\
\hline Healthcare & $\begin{array}{l}\text { Individual and population health } \\
\text { indicators such as absenteeism from } \\
\text { work, physician visits, laboratory tests } \\
\text { and drugs consumption }\end{array}$ & $\begin{array}{l}\text { Early detection of } \\
\text { disease outbreaks }\end{array}$ & $\begin{array}{l}\text { Buckeridge et al. (2005) } \\
\text { Lamma et al., (2006) }\end{array}$ \\
\hline $\begin{array}{l}\text { Computer } \\
\text { Systems }\end{array}$ & $\begin{array}{l}\text { Data trail left by users and system } \\
\text { activity information like number of } \\
\text { accesses, time of day, number of } \\
\text { logins/failures }\end{array}$ & $\begin{array}{l}\text { Unauthorized } \\
\text { intrusion }\end{array}$ & $\begin{array}{l}\text { Lee and Stolfo (1998) } \\
\text { Zhu et. al. (2001) }\end{array}$ \\
\hline Environment & Seismic signals & Earthquakes & Dzwinel et al (2003) \\
\hline
\end{tabular}


The successful application of detection based approaches in a wide range of domains gives confidence that it could be applied in supply chain management applications including rogue seasonality. However, it is first necessary to understand the current status of detection based approaches in supply chain management. Also, SR being an information centric approach, it is also important to understand the nature and extent of information availability within supply chains; and finally, what techniques could use this information to enable detection.

\subsubsection{Status of detection based approaches in supply chain management}

The need for detection based approaches has been recognised by many recent studies on supply chain risk management. For example, Hendricks and Singhal (2003) suggest development of the ability to predict supply chain glitches (or disturbances) as a means of mitigating the negative economic effects of such glitches. According to them "Firms often come to know about problems too late to avoid or minimize the adverse consequences. A desirable capability would be the ability to predict potential glitches. Developing predictive capabilities involves selecting and tracking leading indicators of future business performance; extracting, integrating and transforming data from different systems to generate the leading indicators; delivering information on these indicators on a real-time basis; and providing visibility into the extended supply chain, including internal operations, suppliers, and customers".

Similarly, Craighead et al. (2007), using a multiple-method, multiple-source empirical research design identify early warning capability as key to reducing the severity of the impact of a supply chain disturbance with warning capability defined as "the interactions and coordination of supply chain resources to detect a pending or realised disturbance". According to them the quicker a supply chain disturbance is detected and pertinent information about it communicated to the relevant entities, the more time the supply chain would have to inoculate itself from its negative effects resulting in a reduction in the severity of the impact. They also highlight the need for this warning capability to span multiple echelons and be communicated on an exception basis.

The need for visibility and predictive analysis systems to identify potential problems and ensure quicker response is also highlighted by Handfield et al. (2008) while 
Elkins et al. (2008) suggest the creation of an "exception" event detection and early warning system for real time supply chain operations and supply based management. Inputs from senior managers in logistics and supply chain functions were used to formulate these recommendations. Finally, Bodendorf and Zimmermann (2005) highlight the importance of disturbance detection by describing the behaviours of two companies who faced supplier breakdowns in which one had and the other did not have a detection system. The plots are given in Figure 2.3.

Without disturbance detection
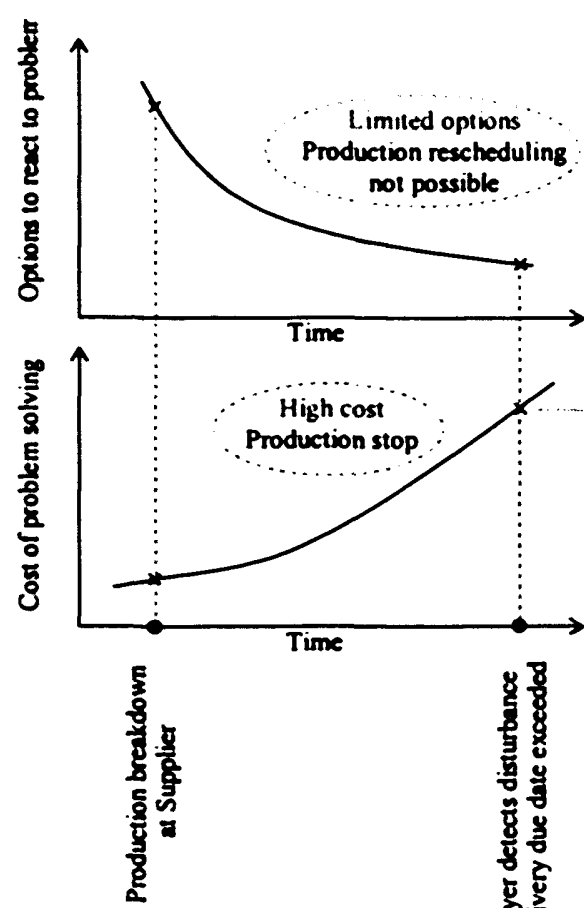

With disturbance detection
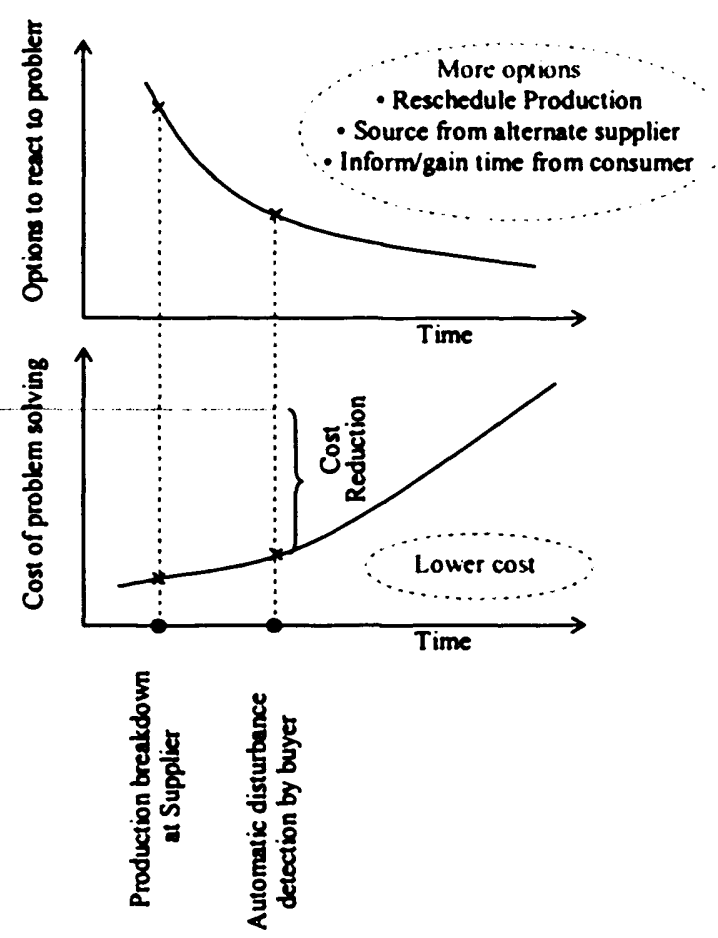

Figure 2.3 Benefits of automatic disturbance detection in a supply chain context (adapted from (Bodendorf and Zimmermann, 2005)

The plots show how with reduction in reaction time, the options to mitigate the impact of a disturbance drop sharply, with a resultant increase in the cost of problem solving. Though these diagrams are not based on real data, the non linear profiles assumed are close to representing what happens in reality. The company without an automatic disturbance detection system knows about the supplier breakdown only when it does not receive the material as ordered. Because all the planning would have been based around processing this material including use of labour and machines, the sudden non arrival of material means fewer options to redeploy these and in an extreme case could result in idling of resources. The adverse cost implication of such an occurrence would obviously be very high. In contrast, the company with an automatic disturbance 
detection system gets greater reaction time, which it uses to reschedule production and/or use alternate source and/or prepare the customer for late arrival, all of which reduce the cost implication of the disturbance.

In terms of the current state of detection approaches, these are not available at the supply chain level at present. For other approaches which are available, their operational aspects are not known. These are discussed below. In this thesis we confine ourselves only to information based automated detection approaches.

Tracking and tracing systems are used by the logistics sector to record and disseminate information relating to product movement and delivery across multiple transportation/warehousing stages and detecting any delays in the same (Hoek, 2002). However, very little analytical processing is involved. The operational scope of disturbance detection in these systems is also restricted to a single logistics service provider (Karkkainen et al., 2004), though there are some recent examples of such systems being used to handle multiple shippers and customers. For supply chains, Supply Chain Event Management (SCEM) systems are being used to detect discrepancies in transaction ordering and order fulfilment processes (Otto, 2003). These are information centric systems normally integrated with ERP systems in which the status of all predefined events are monitored vis-à-vis plans, and in case of deviations exceeding specified thresholds, notifications are issued or corrective actions automatically triggered. The SCEM system is also intra enterprise and lacks intelligence to identify new problems or predict initiation of problems (Bodendorf and Zimmermann, 2005).

Recently, researchers at IBM have tried to incorporate in an SCEM system, both a predictive ability for events/disturbances as well as an action ability to neutralise the impact of disturbances through use of analytical techniques such as simulation, optimisation and data mining (Buckley et al., 2006). They demonstrated the effectiveness of their approach using two pilot case studies: demand conditioning in personal computers and inventory management in a technology supply chain. However, their explanation was at a high level and offered limited insights on information and the analytical techniques used. Finally, prognostic logistics which use real time information to predict the future state of a system (Cruz et al, 2007) and autonomic logistics (Menotti, 2004) which refer to technologies that predict failure in 
operating systems, monitor stockage levels in consumables, automatically report impending failures and order replacements without human intervention are concepts related to detection. However, very few studies have covered these subjects and those that have, have also given only high level descriptions of the application contexts.

Overall, sense or detect and respond seems to be a promising approach for application in supply chain problems, especially those pertaining to disturbances, as highlighted by several recent studies on the subject. Though this approach has been applied at both strategic as well as operational levels and across various domains, its application in supply chain management has been limited and it has been applied within a limited context. Few research studies have discussed detection based approaches in supply chain management and even fewer have provided details about the information and techniques that they used for detection.

\subsubsection{Supply chain information in the context of detection}

Information available for detection is a function of the level of trust and the nature of collaborative relationship among the supply chain partners. It therefore varies across supply chains. At one extreme, there are entities in traditional supply chains sharing only order information, while at the other extreme in a collaborative planning and replenishment (CPFR) relationship, information on multiple variables such as demand, stocks, orders, production, shipments, returns, capacity, capacity utilisation and backlogs is shared (Lee and Whang, 2000; Fliedner, 2003). Other collaborative formats such as vendor managed inventory (VMI) typically share sales and inventory information (Disney and Towill, 2003). While the information sharing at present is predominantly used for improving the replenishment processes, the same could be put to use for detecting disturbances. This has been highlighted by many researchers such

as Tang (2006) who identify information management as a separate category for managing supply chain risks and Elkins et al. (2008) who emphasise the role of visibility of material and information flows for a similar application.

\subsubsection{Generic approaches and techniques of detection}

Given that detection oriented studies in supply chain management did not provide operational details about techniques used, it is important to explore generic techniques 
that could be applied in the present context. There are broadly two kinds of information based approaches for disturbance detection as depicted in Figure 2.4: Change point detection or statistical based and signature based.
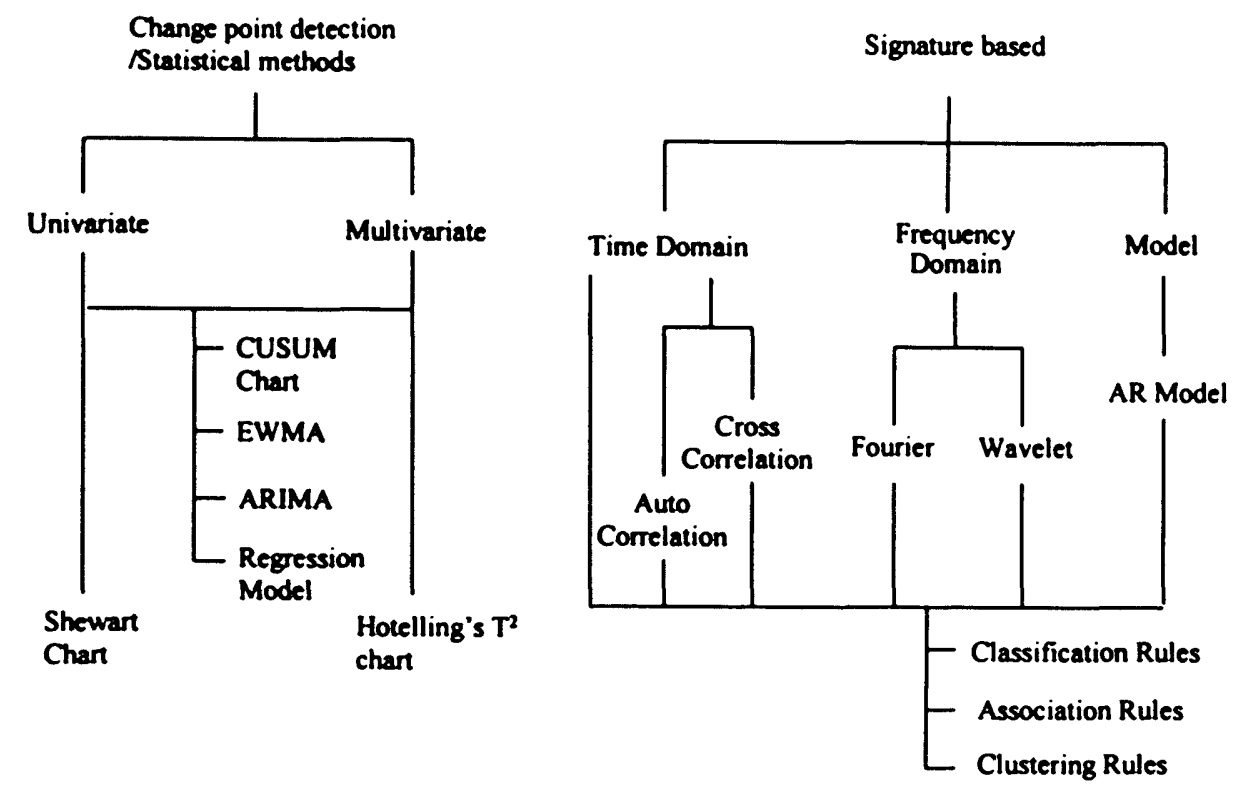

Figure 2.4. Generic disturbance detection techniques

\subsubsection{Change point detection techniques}

Change point detection approaches, which are also referred to as statistical approaches, are based on assessing a change in the profile of a variable in statistical terms such as a change in probability distribution or a change in the parameters of a fitted distribution (Basseville and Nikiforov, 1993). In case of the change being significant based on a specified threshold, the test signal is characterized as a disturbance. This technique has been extensively applied in the manufacturing and process industries (Montgomery, 2005; Venkatasubramanian et al., 2003) after adapting it for different information profiles such as Shewart for stationary time series, cumulative sum or CUSUM for detecting small shifts, exponentially weighted moving average or EWMA for correlated time series, and approaches based on fitting models like ARIMA or regression to data for detecting small shifts and generic time series. Multivariate generalizations of these techniques, where the multiple variables are combined into a mean vector using the correlations between the variables are also being used in detection. 
At the supply chain level use of change point techniques for detection has been quite limited (McCarthy and Wasusri, 2002; Morgan and Dewhurst, 2007). One reason is that supply chains typically involve a large number of variables and the relevant change point detection technique, such as of the multivariate kind which could be applied in such a case, lose their effectiveness with an increase in the number of monitored variables (Woodall and Montgomery, 1999). Secondly, supply chains are cascaded mutli-stage processes and modelling such processes and fixing thresholds for disturbance detection is difficult (Tsung et al., 2008; Batson and Gough, 2007). Fixing thresholds is also diffficult because supply chain variables are typically non stationary with a mix of various trend and seasonality components. Given the difficulties in applying these techniques at a supply chain level for detecting disturbances, focus shifts to the alternative signature based techniques.

\subsubsection{Signature based techniques}

Signature based techniques are based on developing signatures or characteristic profiles of variables associated with different disturbances. A test instance consisting of a profile of relevant variables is compared with these signatures to determine extent of similarity. If found similar, based on a user defined threshold, the test instance is classified as a disturbance of the corresponding type. Signature based techniques are gaining in popularity in various domains in view of their flexibility, as they do not have any constraints in terms of number of variables as in change point detection techniques, and availability of greater variety of tools. In fact all the studies cited in Table 2.2 have used signature based techniques for detection. There are two parts to signature based techniques: a) Techniques to determine signatures or rules of detection from information, b) The nature of information used in these techniques: raw time series or time series which had been transformed into a different domain.

General data mining literature suggests three kinds of techniques for determining signatures or rules: Classification, Association and Clustering (Han and Kamber, 2006; Tan et al., 2005). In the classification technique, a set of pre-categorized data objects are used to develop signatures or rules, which are subsequently used to classify new objects. On the other hand Association rules derive multi variable correlations that is correlating which two events or things are associated together. In the context of disturbance detection this could mean determining what time based 
sequences of events (patterns or distinguishing features) of different variables are frequently encountered with a disturbance. Finally, clustering techniques are concerned with the partitioning of data sets into a small number of homogenous groups or clusters so that data objects in a group share the same properties, while those in different groups are dissimilar. Rules are derived from the nature of clusters formed. Though all the three techniques are used for static data, for time series data, clustering has been used the most (Liao, 2005).

In terms of the nature of information to be used in clustering, use of raw time series, though easier to interpret, creates problems in computation due to large, high dimensional data sets as well as lower accuracy because of interference from noise. The use of alternative time series features has therefore been explored such as amplitudes of Fourier transform (FT) (Agrawal et al., 1993; Caiado et al., 2006), coefficients from an autoregressive (AR) model (Kalpakis et al., 1999; Maharaj, 2000; Ting et al., 2003), wavelet coefficients of discrete wavelet transform (DWT) (Chan and Fu, 1999; Lin et al., 2004; Zhang et al., 2005), autocorrelation function (ACF) (Wang and Wang, 2000; Vlachos et al., 2006) and cross correlation function (CCF) which is a feature of pairs of time series (Bohte et al., 1980; Baragona, 2001; Aono et al., 2006).

The problem with these studies is that most of them have demonstrated the effectiveness of their respective features using synthetic, non contextual data. Since the results and effectiveness of different features is domain specific and none of these studies have used any supply chain related data, their applicability in the supply chain context is limited. Secondly, few studies have compared the performance of their features with other features, and those that have, have used only one or two features in the comparison (Keogh and Kasetty, 2003; Liao, 2005). Hence, applying and testing different time series transformations and features for time series clustering of supply chain data constitutes a gap.

\subsection{Summary}

The subject of disturbances in supply chains has received increased academic as well as practitioner interest in recent times. Researchers have studied this subject from different perspectives such as understanding the source of these disturbances, their 
assessment and management. However, most of them have used a conceptual or empirical approach with quantitative data driven approaches being used by very few researchers. Even the empirical studies have being conducted at a high level using primarily qualitative approaches (Svenssson, 2000; Zsidisin, 2003; Norrman and Jansson, 2004). Such studies provide only a limited usefulness. This is because researchers and practitioners are unable to understand the key variables influenced by/influencing the disturbance, the relationships among them and methodologies or tools to manage them (Blackhurst et al., 2005). Non consideration of realistic multi echelon structures in these studies further limit their practical utility.

A more useful approach is to focus on individual causes of disturbance and investigate them in detail using multi echelon structures and quantitative approaches. This practically relevant approach would help create risk management tools and techniques whose scarcity has deterred companies from adopting risk management practices (Christopher and Lee , 2004; Blackhurst et al., 2008). This approach has been followed in the study.

Disturbances can originate from demand, supply, process, control and environmental sources. Among these, control disturbance, which is endogenously generated from use of inappropriate information and/or control algorithms to match supply with demand (Mason-Jones and Towill, 1998) has a significant negative impact on cost efficiency (Metters, 1997; Lee et. al, 1997a, b) and also affects a large number of supply chains (Geary et. al, 2002; McCullen and Towill, 2002). The two manifestations of control disturbance are, the Bullwhip effect (Lee et al., 1997a, b) in which the variability in orders is amplified from downstream to upstream echelons and rogue seasonality (Forrester, 1961; McCullen and Towill, 2002) in which seasonality in supply chain variables is generated endogenously.

Extensive research has been conducted on the Bullwhip effect. It's manifestation in multiple empirical sectors has been studied (Miragliotta, 2006), its causes such as demand signal processing and lead time (Forrester effect), batching, shortage gaming, price promotion and behavioural have been investigated (Geary et al., 2006), different methods such as statistical, control theory, system dynamics simulation and management games (specifically Beer game) have been used to study it and various 
measures have been proposed for its measurement. On the other rogue seasonality has been investigated in very few studies.

The review of the literature revealed only two studies which were exclusively focused on rogue seasonality. The study by Kim and Springer (2008) applied analytical system dynamics approach to determine the conditions governing the strength of rogue seasonality generated. Specifically, the role of ordering policy parameters, that is, the weights assigned to inventory gap and pipeline gap terms in generating strong and weak cyclicality was assessed. However, the analysis was limited in scope; a dyadic structure was used, the analysis did not assess the impact of forecasting policies on the dynamics and the rogue seasonality strength was inappropriately defined from a subjective perspective with a frequency bias. The study by Kim and Springer (2008) also did not investigate the impact of various relevant factors such as demand processes and parameters, batching, backlogs and different order of delays (Sterman, 2000) on rogue seasonality as has been done for the Bullwhip effect. The second study on rogue seasonality by (Thornhill and Naim, 2006) is empirically oriented and uses the context of a steel supply network to characterise cyclical disturbances in the network. However, this exploratory study used only a single technique for rogue seasonality detection and tested it using only one case study. The technique used was also not automatic and required numerous manual interventions. The study of rogue seasonality is important as it is present in many supply chains (Kaipia et al., 2006; Lee et al., 1997a; Torres and Moran, 2006) and causes significant cost inefficiency as in the case of the Bullwhip effect (Metters, 1997). It therefore needs to be investigated in a rigourous fashion as has been done for the Bullwhip effect.

One approach to manage rogue seasonality is to minimise its generation through appropriate choice of forecasting and ordering policies and parameters. This approach was used by Kim and Springer (2008) who used system dynamics analysis of a dyadic system to determine the conditions (ordering policy parameters) under which strong and weak endogenous seasonality could arise in a supply chain. Such analytical design based approaches have also been proposed for controlling the Bullwhip effect (Lee et al., 1997b; Chen et al., 2000). However, the effectiveness of these approaches in real environments is limited. This is because supply chains in reality are quite complex, because of the dynamic interactions among multiple echelons in an 
uncertain and continuously disturbed environment, which makes them difficult to control (McCarthy and Tan, 2000; Baader and Montanus, 2008). At the same time, appropriate control policies and parameters determined in the studies are too simplistic in relation to this complex reality, having being developed under dyadic settings (Chen et al., 2000; Daganzo, 2004). Moreover, the actual nature of the application of these policies and parameters in real applications is also uncertain. This is because, while in the studies these have been determined on the basis of complete rationality in decision making, in actual practice behavioural biases and significant departures from rationality take place in many decision making situations (Schweitzer and Cachon, 2000; Bendoly and Cotteleer, 2008; Croson et al., 2005; Wu and Katok, 2006). Top down planning therefore needs to be complemented with a bottom-up sense or detect and respond based approach. Instead of uniformly applying generic control policy and parameter prescriptions to all supply chains, the more problematic ones (in terms of rogue seasonality in this case) could be identified (detect) to which generic rules which have been appropriately contextualised are applied (respond).

Sense and respond is a management approach for an unpredictable and dynamic environment It has been applied in various strategic (Haeckel, 1999) as well as operating contexts such as in fraud detection (finance), fault detection (equipment maintenance), disease outbreak detection (healthcare) and unauthorised intrusion detection (computer systems). Anomalies are sensed using system information and relevant analysis techniques on the basis of which management takes action (respond). The utility of the sense and respond approach in the context of supply chain risk management has also been recognised (Bodendorf and Zimmermann, 2005; Hendricks and Singhal, 2003; Craighead et al., 2007; Handfield et al., 2008) though its actual application in this domain has been limited. Few research studies have discussed detection based approaches in supply chain management and even fewer have provided details about the nature of application. This thesis explores the application of the sense and respond approach in rogue seasonality management. In an environment where multiple supply chains are being managed, the more problematic ones in terms of rogue seasonality could be sensed, to which generic rules which have been appropriately contextualised are applied (respond). 
Change point detection or statistical and signature based approaches are the two generic approaches for detection. Change point detection approaches are based on assessing a change in the profile of the variable in statistical terms such as in probability distribution (Basseville and Nikiforov, 1993). It has seen limited applications in supply chain contexts (McCarthy and Wasusri, 2002; Morgan and Dewhurst, 2007) because it is less effective with multi echelon cascaded structures, when the number of variables is large and the variable profiles are non stationary (Woodall and Montgomery, 1999; Tsung et al., 2008) all of which are relevant to supply chains. On the other hand signature based approaches, which are based on developing signatures/profiles of variables that can be associated with a disturbance, have none of the above constraints. Though application of this approach in supply chain management domain has also been limited, it has been applied in other domains such as finance, equipment maintenance, healthcare and computer systems and is gaining in popularity because of its flexibility and greater availability of tools/techniques for application. Signature based approaches were therefore considered for rogue seasonality detection.

Use of signature based approaches involves choosing techniques to determine signatures or rules of detection and the nature of information to be used in these techniques (raw time series or time series which had been transformed/converted into a different domain). The data mining literature suggests Classification, Association and Clustering as the three techniques for determining signatures or rules (Han and Kamber, 2006; Tan et al., 2005) out of which clustering has been used the most for time series data (Liao, 2005). Signatures based on clustering involve finding the similarity/dissimilarity relationships among the time series profiles of the operating variables, allocating them to separate homogenous groups or clusters and relating the profile of clusters to the relevant disturbance. In terms of the nature of information used in clustering, either raw time series could be used or it could be transformed into other domains with features from the transformation used in clustering. Typical features that have been used in time series clustering studies in different domains are amplitudes of Fourier transform (FT) (Agrawal et al., 1993; Caiado et al., 2006), coefficients from an autoregressive (AR) model (Kalpakis et al., 1999; Maharaj, 2000), wavelet coefficients of discrete wavelet transform (DWT) (Chan and Fu, 1999; Lin et al., 2004), autocorrelation function (ACF) (Wang and Wang, 2000; Vlachos et 
al., 2006) and cross correlation function (CCF) which is a feature of pairs of time series (Bohte et al., 1980; Baragona, 2001). However, none of these studies have used supply chain related data. Since the results and effectiveness of different features is domain specific, applicability of findings from these studies to the supply chain context is limited. Secondly, few studies have compared the performance of their features with other features, and those that have, have used only one or two features in the comparison (Keogh and Kasetty, 2003; Liao, 2005). Hence, applying and testing different time series transformations and features in time series clustering of supply chain data constitutes a gap. Here it may be noted that a major limitation in Thornhill and Naim's study was that they did not compare their proposed SPCA technique with other techniques

\subsection{Research Questions}

The focus of this thesis is on applying the sense or detect and respond based approach to manage rogue seasonality which has not been investigated in the past. This requires rogue seasonality contexts which are well understood so that they could be used for assessing the effectiveness of different aspects of detection. Moreover, different contexts are required so that the detection approach is rigourously validated. In contrast, the study by Thornhill and Naim (2006) used just one case study to justify their SPCA detection technique.

Since only a limited number of studies have investigated rogue seasonality, the Bullwhip effect was used as a reference to identify different contexts that could be used. The search was limited to simulation contexts as the exploratory nature of research required detailed and controlled investigation. Two simulated contexts were found to have been extensively used for analysing the Bullwhip effect; one based on linear supply chain models (Towill, 1982; John et al., 1994) and the other based on non-linear Beer game models (Van Landeghem and Vanmaele, 2002; O'Donnell et al., 2006). Since, use of common approaches promotes effective knowledge building, these models were used in the present study. Findings from simulation have high internal validity but their external validity is low. Therefore, empirical rogue seasonality contexts also need to be used in the analysis. 
The second part of the research involved using the different rogue seasonality contexts to establish the best approach for its detection. This includes a signature for rogue seasonality which could indicate its presence, an objective numerical indicator or 'index' of rogue seasonality intensity and the best time series transformation techniques (or features) and variables to be used for their computation. At present there is no 'index' for measuring rogue seasonality though there are multiple measures for the Bullwhip effect. Finally, the ability to sense or detect rogue seasonality needs to be exploited to better manage rogue seasonality in a practical setting which requires a structured managerial framework.

This links to the four research questions that are addressed in the thesis:

1) What signature and index could be used for detecting rogue seasonality in a linear supply chain? What is the best feature (domain representation) and the appropriate system variables for deriving them?

2) Can the signature and index identified from the linear supply chain be applied to a non-linear supply chain with backlogs and batching? Is the best feature and appropriate variables for detecting rogue seasonality in this non-linear system the same as in the case of the linear system?

3) Can the signature and index be used for detecting rogue seasonality in real supply chains? Are the best features and system variables for deriving the signature and index the same as found in RQ1 and RQ2?

4) How can rogue seasonality detection be used in a managerial framework?

Answers to these questions would help establish a robust mechanism for automatic detection of rogue seasonality including identification of supply chains with high rogue seasonality intensities so that management could focus on such cases and effect mitigative action. Automation in rogue seasonality detection was identified to be one of the areas for future research in the study by Thornhill and Naim (2006). 


\section{Chapter 3 - Methodology}

The previous chapter highlighted the research gaps and the relevance of the research questions. This chapter gives details of the methods used to carry out the research, including their explanation, justification and placement within the wider context of alternative methods.

First, the philosophical approaches and methods used in supply chain management research are discussed. Next, the philosophical stance of the thesis and the research design used to answer the research questions are explained. Finally, details of the methods used are provided. These include simulation and case studies for contextual rogue seasonality data and time series transformation techniques and clustering for rogue seasonality detection.

\subsection{Research philosophies and paradigms}

Meaningful and productive research requires a sound and relevant philosophical basis. This is because unconscious research and naive investigative activities rarely lead to more than simply confirming what is already known (Arbnor and Bjerke, 1997). The philosophical position is represented as a research paradigm, which is a central element of the research process and represents the researcher's perspective on the world (Naslund, 2002). The background of the researcher is therefore a significant factor in determining the philosophy underpinning the research.

A research paradigm has three elements: ontology, epistemology and methodology (Denzin and Lincoln, 1994). Ontology focuses on the "nature of reality" or the "nature of knowledge" (Guba, 1990). Defined as the science of being, it refers to the assumptions that a particular approach to enquiry makes about the nature of social reality, specifically whether the reality is viewed from an objective or subjective perspective. Epistemology on the other hand deals with how the world is perceived and is appropriately defined as the science of the methods of knowledge. It refers to the claims or assumptions made about the ways in which it is possible to gain knowledge of the reality. Together, 
epistemological and ontological assumptions influence methodology, which deals with how knowledge about the world is gained. Defined as "a body of methods, procedures, concepts and rules (Merriam-Webster Dictionary 2004), methodology is the rationale or basis for the selection of methods used to gather data. It needs to be appropriate in relation to the research questions and objectives to be addressed.

Positivism and Interpretivism are the two main paradigms used in business research (Bryman and Bell, 2004). Positivism advocates the application of the natural sciences' methods in business research and believes in an objective reality which can be confirmed by the senses, measured and generalized. The quantitative research methodology is usually associated with positivism and is characterized by a numerical orientation and emphasis on the measurement and analysis of causal relationships between variables (Saunders et al., 2002). The Interpretivism paradigm on the other hand holds an opposite view to Positivism: reality is not considered external to the actors as in positivism but a part of the subjective interpretation of the actors themselves. Human beings and their interactions are considered to be distinctive, with the principles of natural science not considered applicable for social systems. Qualitative research methodologies (Saunders et al., 2002) are usually associated with the Interpretivism paradigm, where there is greater emphasis on human behaviour and its role in the research context.

While the above provides a generic explanation about research philosophies and paradigms, it is important to understand which of these philosophies have been used in supply chain management research.

\subsection{Nature of research in supply chain management}

Supply chain management is an interdisciplinary subject. It incorporates many different scientific traditions (Arlbjørm and Halldorsson, 2002), has been influenced by economic and behavioural approaches mainly through the business disciplines of marketing and management (Mentzer and Kahn, 1995), but also borrowing from engineering and computing (Stock, 1997). The dominant stream of research has therefore been based on the positivistic paradigm similar to that for economics and engineering. This is borne out by the literature surveys on research methods used in logistics and supply chain 
management by Mentzer and Kahn (1995), Frankel et al. (2005) and Sachan and Datta (2005) as given in Table 3.1. The most used research methods of survey, simulation and mathematical modeling all come under the positivistic paradigm.

\section{Table 3.1: Research methods applied in logistics and supply chain management}

\begin{tabular}{|c|c|c|c|}
\hline Category & 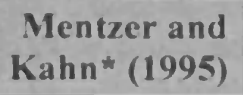 & $\begin{array}{l}\text { Frankel et al., } \\
\qquad(2005)^{* *}\end{array}$ & $\begin{array}{r}\text { Sachan and } \\
\operatorname{Datta}^{\wedge}(2005)\end{array}$ \\
\hline Survey & $54 \%$ & $37 \%$ & $35 \%$ \\
\hline Simulation & $15 \%$ & \multirow{2}{*}{$15 \%$} & $5 \%$ \\
\hline Math Modelling & $4 \%$ & & $10 \%$ \\
\hline Interviews & $14 \%$ & $19 \%$ & $7 \%$ \\
\hline Case Studies & $3 \%$ & $7 \%$ & $16 \%$ \\
\hline Archival Studies & $10 \%$ & $7 \%$ & \multirow{2}{*}{$27 \%$} \\
\hline Others & $0 \%$ & $15 \%$ & \\
\hline
\end{tabular}

- \% of articles in Journal of Business Logistics (1978-1993)

- \% of articles in Jowrnal of Business Logistics (1998-2003)

A \% of articles in Journal of Business Logistics, International Journal of Physical Distribution and Logistics Management and Supply Chain Management - An International Journal (1999-2003)

\subsection{Philosophical stance of the thesis}

Each of the two main components of the thesis, rogue seasonality generation and rogue seasonality detection, determine the philosophical stance to be used in the thesis. Rogue seasonality has a similar origin as demand amplification or the Bullwhip effect, which has mostly been studied under the positivistic philosophy using quantitative methodologies (Geary et al., 2006). The positivistic philosophical approach could therefore be used to study rogue seasonality generation as well. With regard to detection of rogue seasonality, most generic techniques of detection are derived from the Mathematics/Engineering/Computer science domains, which have a quantitative orientation and are based on positivism. Therefore on both counts, positivism seems to be an appropriate philosophy for conducting the research envisaged in the thesis. On the other hand, the alternative interpretivism philosophy was considered inappropriate because rogue seasonality disturbances are not based on judgement but are actually 
generated. Similarly, rogue seasonality assessment and measurement are considered to have an objective and numerical basis rather than being subjective.

Another factor which affects the philosophical underpinnings of research is the background of the researcher. Although the author has conducted research based on both positivism and interpretivism philosophies, his leanings are more towards the former. Research in this thesis has therefore been undertaken from a positivistic perspective. Details of the process used for conducting the research are explained in the following sections.

\subsection{Research design}

Research design is a framework to conduct research that links the data to be collected and analysed for answering the research questions. It provides the opportunity for "building, revising and choreographing" (Miles and Huberman, 1994) the overall research study. The choice of research design reflects decisions about the priority being given to a range of dimensions of the research process (Bryman and Bell, 2004). The overall framework used in the thesis is given in Figure 1.1 but adapted in Figure 3.1 below for further clarification.

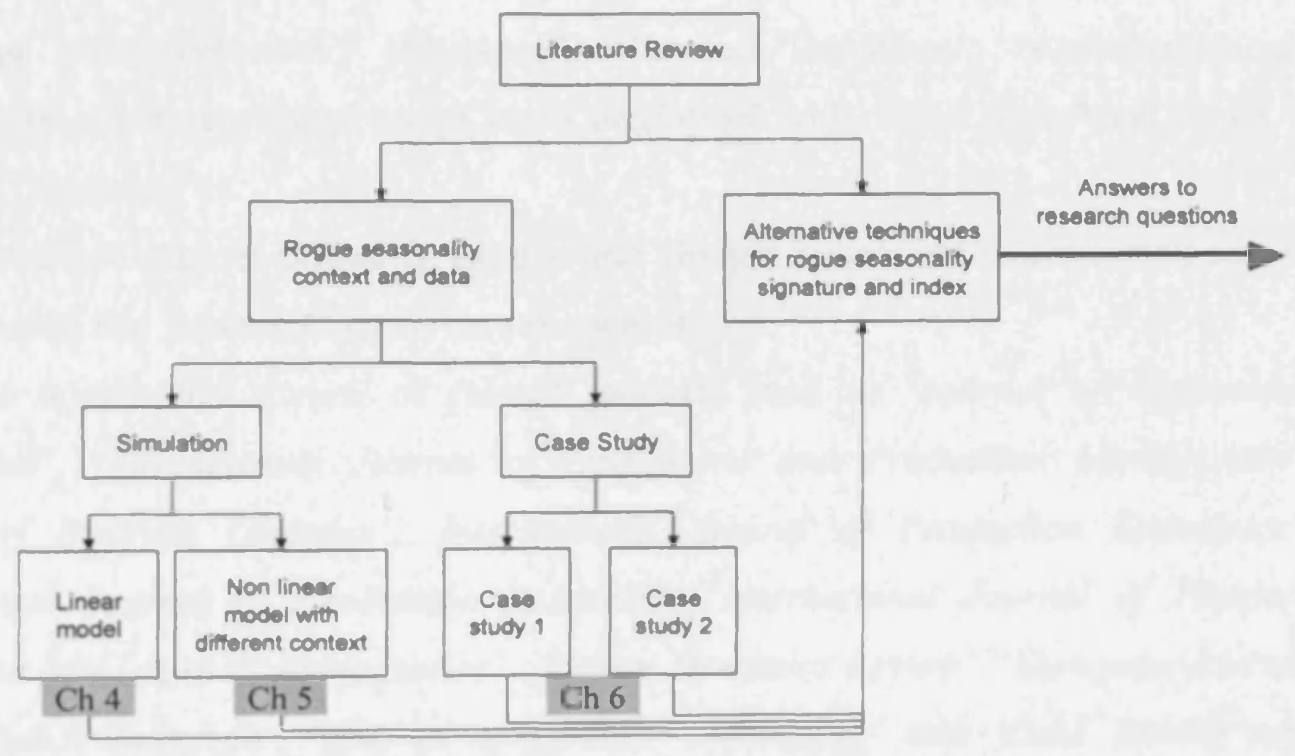

Figure 3.1 Research framework used in the thesis 


\subsection{Literature review process}

The literature review is the starting point as well as the foundation of most research processes (Saunders et al., 2002). It is used to understand the current state of knowledge of the subject, its limitations and to generate and refine ideas to fit with the research context under study. The nature of the research in this thesis being of an exploratory and innovative nature, an exhaustive literature search was conducted. The different sources used in the search include:

a) Key word searches in all the major databases such as Scopus, Proquest, Emerald, IngentaConnect and Science Direct. An indicative list of terms used are given below:

- 'Risks', 'Disturbances', 'Uncertainties', 'Vulnerabilities', 'Disruptions' and 'Failures' with 'supply chains', 'logistics' and 'supply networks'

- 'Rogue seasonality' 'Endogenous cyclicality', 'Bullwhip', with 'logistics' and 'supply chains'

- 'Statistical process control', 'Failure mode and Effects analysis (FMEA)', 'Decision support systems', 'Knowledge discovery', 'Artificial intelligence', 'Data mining', 'Risk monitoring', 'Event Management', 'Sense and Respond', 'Adaptive', 'Autonomic' with 'logistics' and 'supply chains'

- 'Change point detection', 'Signature', 'Fourier Transform', 'Autocorrelation', 'Wavelets', 'Autoregressive' and 'Cross Correlation' with 'Time series' and 'Time series Clustering'

b) Internet search engines primarily Google and Google Scholar to discover and access the latest books and presentations on the research subject.

c) Periodic access and review of related journals such as 'Journal of Operations Management', 'International Journal of Operations and Production Management', 'Journal of Business Logistics', 'International Journal of Production Economics', 'International Journal of Production Research', 'International Journal of Physical Distribution and Logistics Management', 'System Dynamics Review', 'European Journal of Operational Research', 'Journal of Quality Technology' and 'Data Mining and Knowledge Discovery' so as to include relevant studies which could have been missed in the key word search. 
d) Tracing back references

e) Accessing the websites of key individuals who are active in the field for working papers and presentations.

\subsection{Rogue seasonality context and data}

A multi-methodology approach was used as such approaches provide methodological triangulation and increase the validity of the findings (Jick, 1979; Seaker et al., 1993; Shukla et al., 2009; Boyer and Swink, 2008). Such approaches are quite prevalent in logistics and supply chain management research (Frankel et al., 2005). The multimethodology approach used in the thesis combined simulation with case studies. Simulation enables controlled experimentation and, therefore, findings from it have high internal validity. However, its use of simplified models make it less realistic from a real world perspective, that is, it gives lower external validity. On the other hand, case study involves the investigation of contemporary phenomenon within a real life context, that is, without any investigator control. Hence, when used together, simulation and case study methods complement each other effectively, by enabling cross-validation of results and allowing the investigator to assess whether generic findings occur in reality and viceversa.

\subsubsection{Simulation}

Simulation is defined as "the process of designing a model of a real system and conducting experiments with it either to understand the behaviour of the system, or to evaluate various strategies to improve the operation of the system" (Shannon, 1975). Simulation has many advantages such as facilitating what-if testing on alternative supply chain scenarios within controlled conditions and the flexibility of compressing and expanding time to understand the dynamic behaviour of a system (Law and Kelton, 2000). According to Banks et al. (1999), simulation is an appropriate tool for studying internal interactions in complex systems or subsystems, providing valuable insights into the effect of each variable and highlighting those likely to have a large impact. Simulation is among the most commonly used techniques in operations research and management (Law 
and Kelton, 2000) as well as in logistics and supply chain management research as is evident from Table 3.1 and also highlighted by Terzi and Cavalieri (2004).

There is a historic precedent of using simulation to study supply chain dynamics including both Bullwhip effect and rogue seasonality (Forrester, 1961). In the specific context of this thesis and answering the research questions, simulation provided controlled experimentation, where parameters in the simulation model could be varied and rogue seasonality of different intensities and characteristics generated in the profiles of variables. This data, with known rogue seasonality intensities, could subsequently be inputted into alternative time series transformation techniques to evaluate their individual effectiveness in rogue seasonality detection.

Different kinds of simulation have been developed to cater to different requirements. For studying supply chain dynamics, the two major simulation approaches that have been used are discrete event simulation (DES) and systems dynamics or SD (Riddalls et al., 2000; Kleijnen, 2005; Akkermans and Dellaert, 2005).

\subsubsection{Discrete event simulation (DES)}

Discrete event simulation (DES) is used for analysing discrete event dynamic systems (DEDS), which usually consist of jobs and resources. In a typical simulation, jobs (individual physical entities in a majority of applications) travel from resource to resource through a series of queues and activities in a stochastic fashion, where their onward progress through the system is determined. For example, in a model of a supply chain, the jobs are raw materials that progress through machines and buffer inventories (both resources) where their attributes are changed and they arrive at the retailer as finished goods. Discrete event simulation models are often used for systems where entities are processed in a linear fashion with limited feedback (Sweetser, 1999; Tako and Robinson, 2009). They are considered more appropriate for discrete processes and those which involve detailed operational analysis of a specific, well-defined system, such as a production line or call centre; systems change at specific points in time: resources fail, operators take breaks, shifts change, and so forth (Greasley, 2004). Discrete event 
simulation has been similarly applied in supply chain domain also (Terzi and Cavalieri, 2004).

This thesis with its focus on rogue seasonality has a supply chain or multi echelon orientation. Also, feedback loops play an important part in the generation of rogue seasonality (Kim and Springer, 2008). Discrete event simulation is less suited on both these aspects as discussed in the previous paragraph. It was therefore not used in the thesis.

\subsubsection{Systems dynamics (SD) simulation}

Systems Dynamics (SD) simulation was advocated as a method of investigating dynamical effects in large, complex systems by Forrester (1961) and involves simulation in continuous or pseudo-continuous time. It simulates aggregate behaviour and is best suited for problems where feedback plays a significant role in determining the dynamic behaviour of the system (Akkermans and Dellaert, 2005). Structures with many echelons as well as those with complex, non linear relationships between variables can be studied using SD simulation.

A typical SD simulation involves representing the relationships between variables as causal loop diagrams, converting them into differential equations, subjecting the system to an exogenous shock and then visually studying the output profiles of variables to understand cause-effect relationships. Additionally, alternative scenarios are tested by adjusting the parameters of a system, adding new linkages and feedback loops or rearranging components of the system to assess their impact on the dynamic profiles and cause effect relationships in the system.

The causal loop diagram (CLD) used in SD simulation depicts the linkages and feedback loops among the elements in the system, as well as between the system and its operating environment which helps the decision-maker in understanding a complex, inter-related system. The specific characteristics of CLD representation are:

- Variables in a CLD are connected by causal links, represented by arrows, indicating the influence amongst the variables 
- In each causal link, the variable at the tail of the arrow is the independent variable while the variable at the head of the arrow is the dependent variable

- A positive $(+)$ causal link means that when the independent variable increases (decreases), the dependent variable increases above (decreases below) what it would have been if the independent variable did not change.

- A negative (-) causal link means that when the independent variable increases (decreases), the dependent variable decreases below (increases above) what it would have been if the independent variable did not change.

The underlying mathematical relationships driving the simulation (differential and algebraic equations) are developed after transforming the CLD into an equivalent level and flow structure or block diagram in control engineering. The four important concepts related to level and flow suggested by Forrester (1961) and which are used in formulating the simulation equations are:

1. Levels which describe accumulations within the system and are drawn as tanks. Levels represent the present values of the variable they contain that have resulted from the accumulated difference between inflows and outflows. For example, in the case of production-inventory control, inventory is a level determined by the inflow of goods produced and outflow of goods sold

2. Flows, which transport the content of one level to another. For example, the inflow of goods produced and outflow of goods sold, as discussed earlier

3. Decision functions, which control the rates of flows between levels (drawn as valves). For example, in the case of production-inventory control, the rate of issue of production orders is a decision function

4. Information channels, which connect the levels to the decision functions. For example, the levels of inventory and WIP could be used to decide the production order rate decision

It is notable that since SD models simulate aggregate dynamics, rates such as order rate, and production rate are used, rather than orders or production quantities. The algebraic and differential equations from the level and flow structure are generally solved using numerical techniques to generate the simulation output profiles. The discretisation required in numerical solution techniques causes SD simulations to be pseudo-continuous 
rather than continuous. While specialist packages such as Vensim $\circledast$, Prosim $\circledast$ and iThink $\otimes$ have been developed for SD simulation, the same can also be done in a spreadsheet where the difference equations could be coded.

This thesis is focused on rogue seasonality which is endogenously generated from the feedback loops in the replenishment-inventory control system as discussed in the literature review. Also, the cantrol system is operative at an aggregate level. Simulation based on system dynamics is appropriate on both these counts. Moreover, SD simulation has been used to understand and model the dynamics associated with the related bullwhip effect (Angerhofer and Angelides, 2000; Akkermans and Dellaert, 2005; Potter and Disney 2006; Villegas and Smith 2006; Wilson, 2007). SD simulation was therefore used to understand rogue seasonality generation as well as for generating related contextual data.

Although SD simulation has been extensively used and has many advantages, it has one major drawback. It does not have a theoretical basis or analytical support for deciding how to vary parameters, feedhack loops and components to improve system performance (Ansoff and Slevin, 1968). Simulation essentially proceeds on a trial and error basis and is therefore cumbersome, time consuming and provides limited insight. Control theory has been suggested as a way to strengthen the theoretical base of SD simulation.

\subsubsection{Control theory and its use in production-inventory problems}

Control theory has been used to study production-inventory dynamics in its own right independent of SD simulation (Ortega and Lin, 2004). Used extensively in the past for studying engineering systems (Nise, 1995), these learnings were subsequently applied to production-inventory control problems using the analogous dynamics between the two contexts. Control theory is typically used in production-inventory control systems by mathematically representing the causal relationships and feedback loops in the system in terms of differential equations or differential equations transformed by Laplace transform to the continuous ' $s$ ' domain or differential equations transformed by the $z$ transform to the discrete ' $z$ ' domain and using these representations to analyse dynamics of relevant variables (Ortega and Lin, 2004; Sarimveisa et al., 2008). Generally, simple structures 
(linear and with one or two echelons) are analysed using control theory. Also, analysis is at an aggregate level (similar to system dynamics) and with deterministic inputs (Ortega and Lin, 2004). A comprehensive list of reasons for using control theory and transfer function techniques in production and inventory control is given in Disney and Towill (2002). (Transfer function is used to represent the dynamics of the system mathematically; it algebraically relates a system's output to its input in the 's' (using Laplace transform) or ' $z$ ' ( $z$ transform) domain and is essentially a polynomial representation in ' $s$ ' or ' $z$ ) The reasons provided by Disney and Towill (2002) are:

- Block diagram, an intrinsic part of control theory, provides a simplistic representation of the casual relationships between system components and helps identify important system structures (Nise, 1995).

- Use of standard forms and notations in control theory simplifies benchmarking and promulgation of models describing best practice (Towill, 1970). It enables adoption of best practices and parameters from hard (engineering) systems (Towill, 1982).

- Use of transfer function simplifies the capture and representation of the dynamics of the system (Popplewell and Bonney, 1987)

- Standard control theory techniques enable dynamic performance metrics of systems such as settling time, peak overshoots and rise times to be calculated without recourse to simulation (Nise, 1995)

- Problems could be transferred across domains (time, ' $s$ ', ' $z$ ', ' $\omega$ ) to exploit benefits of operating in those domains (Disney and Towill, 2002). For example, conversion to frequency $(\omega)$ domain enables easier analysis of systems with cyclical characteristics. It also enables simpler computation, as the dynamics of multi echelon structures can be easily computed by straight forward vector multiplication for linear systems. (Bissell, 1996). Moreover, frequency response analysis could be used to examine the critical design parameters and identify ranges of parameter values that give good transient response performance (Ortega and Lin, 2004)

- Transfer functions could be integrated with simulation for additional system analysis (Disney and Towill, 2002). 
The major studies applying control theory in production-inventory control problems, include the study by Tustin (1953) who first explored the application of control theory in the design of 'soft' systems, where the analysis included modelling of an economic system. Valuable insight into a production control system was given by Simon (1952) using a basic servo mechanism theory by considering a continuous time model in the ' $s$ ' domain. He described a simple system for inventory control using feedback loops and linear differential equations to control the rate of production for a single product. Subsequently, Vassian (1955) used a discrete model for the analysis of an inventory control system using difference equations and the application of ' $z$ ' transforms. Axater (1985), who reviewed earlier research on control theory applications in production and inventory control, highlighted the fact that dynamic effects and the importance of feedback are illustrated extremely well by the use of control theory. Ortega and Lin (2004) and Sarimveisa et al. (2008) have reviewed recent research on the application of control theoretic methods to production-inventory systems. Previous work on application of transfer function techniques in the field of production and operations management has been summarised by Disney and Towill (2002).

An important contribution in control theory, especially with regard to its use to strengthen the theoretical base of systems dynamics was made by Towill (1982). Instead of using a complex model with repeatedly coupled non-linear linkages like Forrester (1961), he split the model into more elementary linear two echelon systems, which allowed a greater depth of analysis, whilst still capturing the salient attributes of system behaviour (Berry et al., 1995). The analysis was based on a combination of control theory and systems dynamics. An early guide on use of this combined approach is given in Edgehill and Towill (1989) who state that although control theory requires making assumptions regarding linearity, basic understanding of system behaviour from control theory techniques enhances the insights obtained from subsequent simulation for production inventory control problems. Since then, a series of investigations based on this combined control theory and simulation based approach (Naim and Towill, 1994) have been carried out and has led to the development and analysis of different control systems as well as different ways of utilizing control theory to complement systems dynamics. 
In this thesis, the combined control theory and simulation approach using simple structures is used for rogue seasonality analysis. Such an approach provides better understanding of the dynamics of variables compared to Forrester's approach of using large scale complex models. The understanding gained from the simple structures could be transferred to larger, more complex structures.

\section{a) Control system variants and the choice of Automatic Pipeline Inventory and Order Based Production Control System (APIOBPCS)}

The first control system analysed by Towill (1982) was the Inventory and Order based Production Control system (IOBPCS), a periodic review algorithm for issuing orders into a supply pipeline, where orders placed are based on average consumption over a period of time and a fraction of the difference between desired and actual inventory (inventory deviations or error). IOBPCS is representative of industrial practice in the UK (Coyle, 1977). Towill was concerned with finding a good solution to balance production adaptation and inventory costs using "best practice" analogues from hard control system theory, and used the latter together with simulation in the study. Since then, different variants of IOBPCS have been developed with all of them designed via the use of control theory together with simulation. Details of these variants are given in Disney and Towill (2005) with each of them constructed by defining some or all of the following five components:

- The lead time, which represents the time between placing an order and receiving the goods in inventory. In manufacturing sites, lead time incorporates production delays while in distribution lead time is from order transmission and shipment delays

- The desired inventory setting, which can either be fixed or a multiple of current average sales rates

- The demand policy, which in essence is a forecasting mechanism that averages the current market demand. The demand policy is a feed-forward loop within the replenishment policy

- The inventory policy, which is a feedback loop that controls the rate at which inventory deficit (difference between desired inventory and actual inventory) is recovered 
- The pipeline policy, which is a feedback loop that determines the rate at which WIP deficit (difference between desired WIP level and actual WIP level) is recovered

In this thesis, different variants of the Automated Pipeline IOBPCS or APIOBPCS model have been used (John et al., 1994; Mason-Jones et al., 1997). This is a general, versatile model which has been applied in multiple contexts. It mimics the heuristics used by humans to replenish inventory (Sterman, 1989; John et al., 1994), has been shown to replicate industrial practice (Evans et al., 1997), represents the logic contained within MRP systems (Fowler, 1999) and can represent lean and agile supply chains (Towill et al., 2001), order-up-to policy (Dejonckheere et al., 2003), vendor managed inventory systems (Disney and Towill, 2003) and remanufacturing systems (Tang and Naim, 2004). The causal loop diagram of the APIOBPCS model is given in Figure 3.2, with Appendix A detailing its block diagram representation and difference equations for a single echelon model.

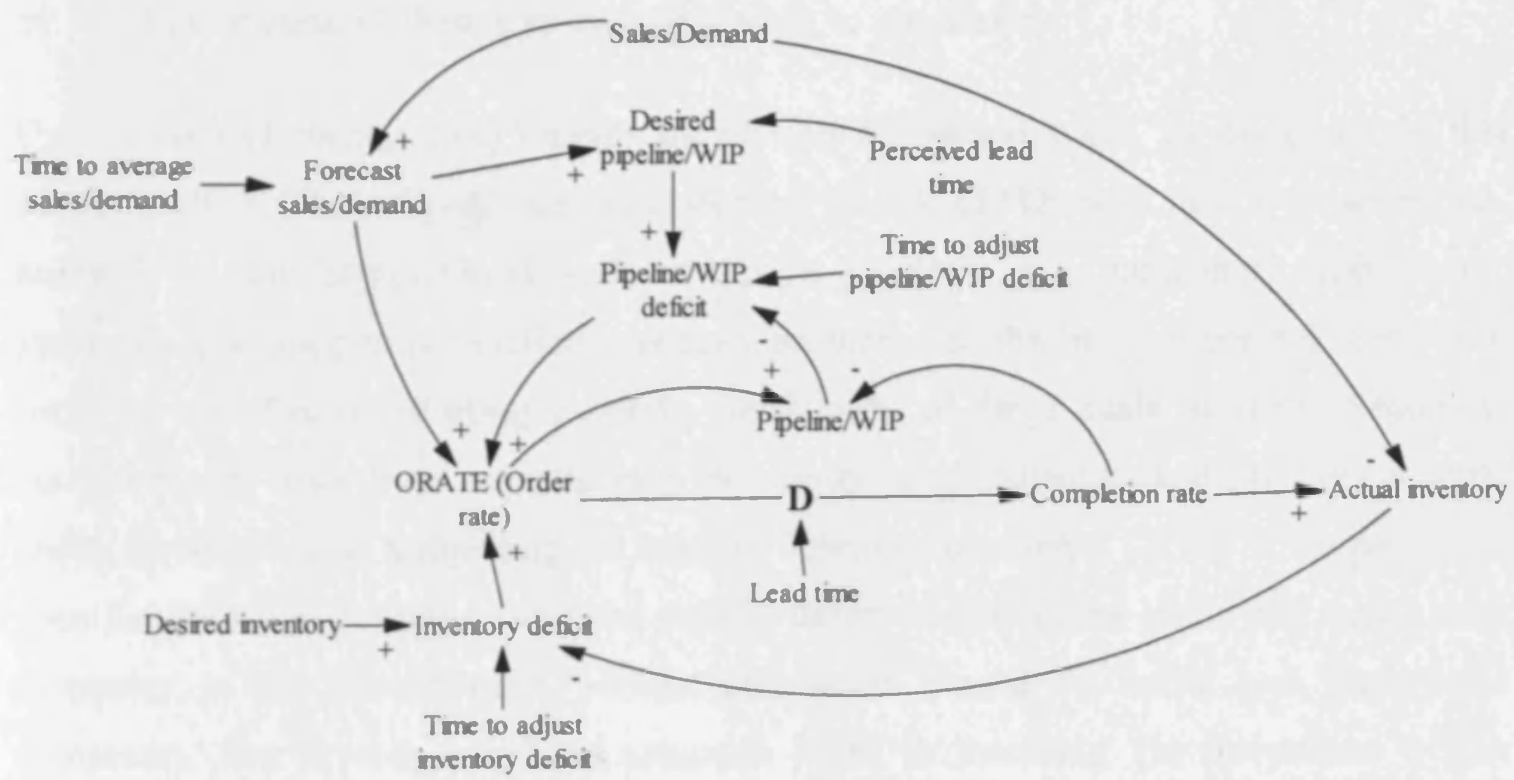

Figure 3.2 Causal loop diagram of the APIOBPCS model (John et al., 1994)

The ordering policy in APIOBPCS can be described as follows; "the order placed is equal to the average sales rate plus a fraction $\left(1 / T_{i}\right)$ of the inventory error plus a fraction $\left(I / T_{w}\right)$ of the work-in-process (WIP) error" where $T_{i}$ is termed the "time to adjust inventory" and $T_{w}$ the "time to adjust WIP". Consumption/sales/demand rate (CONS), forecast 
consumption/sales/demand rate (AVCONS), order rate (ORATE), work in progress level (WIP), desired work in progress level (DWIP), production completion or receipts into inventory rate (COMRATE), actual inventory level (AINV), error between desired and actual inventory level (EINV) are the system variables in a single echelon APIOBPCS system. In terms of parameters used, average sales rate is calculated using exponential smoothing and is dependent on a parameter $T_{a}$ related to the exponential smoothing parameter $\alpha$. While $T_{p}$ is a physical parameter, $T_{i}, T_{w}$ and $T_{a}$ are decision parameters whose values are set according to performance criteria such as the minimisation of order variance, inventory availability and the speed of response to changes in demand. John et al. (1994) proposed parameter values which gave good dynamic performance in an APIOBPCS system and these are given in Appendix A. The focus of this thesis is, however, on generation of rogue seasonality rather than getting a good dynamic performance and, therefore, other parameter values are also applied.

\section{b) Use of control theory as a complement to simulation}

Use of control theory complements simulation in several ways, as discussed in this section earlier. The original use suggested by Towill (1982) was that it removes 'adhocism' in simulation. Good systems design practices and parameters from 'hard' systems are applied to production inventory problems on the basis of control theory and serve as an effective starting point in simulations of large scale systems. Secondly, control theory also gives insights into the steady state dynamics and provides a cross check for simulation. Subjecting the transfer functions of interest (' $s$ ' or ' $z$ ' domain) of a specific system to limiting conditions enables determination of the initial and steady state dynamics in the time domain without simulation (based on initial and final value theorems). The inverse transform approach helps in assessing the correctness of the simulation formulation. The system is subjected to a deterministic input and the analytical response (of different variables) obtained by inverse transforming the function is compared to the simulation output, with a good correlation between them, indicating the correctness of the simulation formulation. John et al. (1994) used initial and final value theorems as well as the inverse transform approach to establish the validity of their simulation output for the APIOBPCS system. 
In this thesis, control theory is used to complement simulation but in a different way to that described above. Transfer functions in the ' $s$ ' (continuous) domain are computed using the Laplace transform first. This ' $s$ ' domain representation for APIOBPCS is given in Appendix B. Alternatively, ' $z$ ' (discrete) domain using $z$ transform could have been used, as findings from both ' $s$ ' and ' $z$ ' domains are qualitatively similar (Disney et al., 2006). However, algebraic manipulation based on the ' $s$ ' domain is preferred because of its tractability (Dejonckheere et al., 2003) and was therefore used. Thereafter, ( $j \omega$ ') was substituted for ' $s$ ' in the transfer function/s and its absolute value in terms of ' $\omega$ ' (angular frequency) determined. Called the frequency response (FR) or amplitude response (Amp R) (both FR and Amp $R$ will be used interchangeably in the rest of the thesis) this function gives the ratio of amplitudes of sinusoidal output to sinusoidal input at various frequencies $(\omega)$. Effectively, by this approach, the transfer function in the ' $s$ ' domain is converted to the frequency (' $\omega$ ') domain as the latter is more suited for data with cyclical characteristics. FR derives its usefulness because of two facts: First, a sinusoidal input to a linear system at a particular frequency yields a sinusoidal output at the same frequency (though with a different amplitude and phase) (Towill and del Vecchio, 1994; Dejonckheere et al., 2003; Jaksic and Rusjan, 2008) and second every time series consists of and can be broken up into its constituent sinusoids at different frequencies (using Fourier transform). Hence, analytically computed FR highlights the input frequencies that would be amplified (FR $>1$ ), attenuated (FR $<1$ ) and the extent of amplification/attenuation, independent of the nature of exogenous input. In this thesis, FR is analysed at critical frequencies such as zero frequency $\left(\omega_{0}\right)$, frequency at which FR is maximum $\left(\omega_{\max F R}\right)$, frequency at which crossover from amplification to attenuation takes place or FR $=1$ ( $\left.\omega_{\text {crossover }}\right)$ and very high frequency $\left(\omega_{\infty ;} \infty\right.$ is used as surrogate for high) for each unique variable in the control system. Such an approach provided adequate insights on rogue seasonality generation and more importantly its characteristic signature that could be used subsequently, for its detection in an unseen setting. Detailed analysis of individual transfer functions was therefore not performed, especially since the focus of this thesis is on the latter, rather than the former. 
The manual computation of the transfer function and its conversion to the frequency domain being quite unwieldy, Matlab® symbolic toolbox was used for the same. Following the control theory analysis, simulation is used to generate contextual rogue seasonality data which is validated by comparing it with the analytically derived FR values at critical points such as $\omega_{\max }$ FR.

\subsubsection{Simulation as applied in the thesis}

The motivation of using simulation was to understand rogue seasonality generation and to generate rogue seasonality data of different characteristics and intensities which could be individually applied to different time series techniques/features to assess their effectiveness in detecting rogue seasonality. The overall simulation design used is summarized in Table 3.2 below.

A sequential modelling process, moving from simpler to more complex structures was used to enable an easier understanding of the rogue seasonality dynamics (single echelon linear to three echelon linear to four echelon non linear). Use of different structures also strengthened the robustness of findings with regard to rogue seasonality signature, index and best techniques for rogue seasonality detection. This explains the logic of research questions 1 and 2.

Table 3.2 Simulation design used for rogue seasonality analysis

\begin{tabular}{|c|c|c|c|}
\hline $\begin{array}{l}\text { Supply chain } \\
\text { structure }\end{array}$ & Structure details & Control system* & $\begin{array}{l}\text { Other variants used in } \\
\text { simulation }\end{array}$ \\
\hline \multirow[b]{2}{*}{$\begin{array}{l}\text { Linear } \\
\text { (Chaprer 4) }\end{array}$} & \multirow[b]{2}{*}{$\begin{array}{l}\text { Single echelon, three } \\
\text { echelon }\end{array}$} & MTO (Make to order) & \multirow{2}{*}{$\begin{array}{l}\text { Different demand } \\
\text { processes and } \\
\text { parameters; Lead } \\
\text { time/delay and } \\
\text { distribution of lead time }\end{array}$} \\
\hline & & MTS (Make to stock) & \\
\hline \multirow{2}{*}{$\begin{array}{l}\text { Non linear } \\
\text { (Chapter 5) }\end{array}$} & \multirow{2}{*}{$\begin{array}{l}\text { Beer game (Sterman, } \\
\text { 1989), a four echelon } \\
\text { distribution structure }\end{array}$} & $\begin{array}{l}\text { Hybrid MTO-MTS with } \\
\text { Optimal parameters }\end{array}$ & \multirow{2}{*}{$\begin{array}{l}\text { Different demand } \\
\text { processes and } \\
\text { parameters; Lead } \\
\text { time/delay; Batching } \\
\text { with different batch } \\
\text { sizes/ No Batching }\end{array}$} \\
\hline & & $\begin{array}{l}\text { Hybrid MTO-MTS with } \\
\text { Unoptimal parameters }\end{array}$ & \\
\hline
\end{tabular}

* Derived from generic APIOBPCS by change of parameters 
For a linear system, multi echelon structures were created by coupling together similar single echelon structures (consumption rate of an echelon was made equivalent to the order rate at its downstream echelon) while the well known Beer game (Sterman, 1989) was used for simulating the non linear structure. The Beer game was used for simulation, as it represents a typical four echelon production-distribution system (retailer, wholesaler, distributor and factory) with ordering and shipment delays across echelons and non linearities from backlogs. The Beer game has been simulated in many different contexts as given in Table 3.3.

Table 3.3 - Studies which have used Beer game simulation

\begin{tabular}{|c|c|}
\hline Reference & Research context \\
\hline van Ackere et al. (1993) & $\begin{array}{l}\text { Assessing impact of ordering delay reduction, echelon elimination and } \\
\text { sharing of demand information sharing on Bullwhip }\end{array}$ \\
\hline Larsen et al. (1999) & Generation of chaotic behaviour from ordering policies \\
\hline Hong-Minh et al. (2000) & $\begin{array}{l}\text { Impact of alternative policies such as EPOS, centralised stock control by } \\
\text { factory ,bypassing an echelon and echelon elimination on lateral emergency } \\
\text { transshipments }\end{array}$ \\
\hline De Souza et al. (2000) & $\begin{array}{l}\text { Impact of shortage gaming, capacity constraints, information and material } \\
\text { delays, poor coordination, demand signaling and order batching on supply } \\
\text { chain dynamics }\end{array}$ \\
\hline $\begin{array}{l}\text { Van Landeghem and } \\
\text { Vanmaele (2002) }\end{array}$ & $\begin{array}{l}\text { Relevance of robust supply chain planning i.e. planning which incorporates } \\
\text { uncertainties; Stochastic demand used with stochastic factory breakdowns and } \\
\text { repairs, stochastic product spoilage and four alternative decision policies }\end{array}$ \\
\hline O'Donnell et al. (2006) & $\begin{array}{l}\text { Use of genetic algorithm to determine optimal ordering policy for individual } \\
\text { echelons }\end{array}$ \\
\hline Paik and Bagchi (2007) & $\begin{array}{l}\text { Significance of demand forecast updating, order batching, rationing and } \\
\text { shortage gaming, price variation, material and information lead time, machine } \\
\text { breakdown, capacity limitations and number of echelons on the Bullwhip } \\
\text { effect }\end{array}$ \\
\hline Hwarng and Xie (2008) & $\begin{array}{l}\text { Investigation of chaos from factors such as demand pattern, ordering policy, } \\
\text { demand information sharing and lead time }\end{array}$ \\
\hline Shukla et al. (2009) & Investigation of shipment dynamics or "backlash" effect \\
\hline
\end{tabular}

While in the role playing version of the Beer game, decisions on order quantities from respective upstream echelons are made by human participants, in the simulated version as used in the thesis, APIOBPCS and its variants are used for the same. According to 
Sterman (1989), the generalised decision rule represented by APIOBPCS characterizes experiential decisions well, as it captures the essential attributes of any minimally sensible stock management procedure consisting of replacement of expected losses, correction of discrepancies between the desired and actual stock and a consideration of the work in progress. From his analysis of the Beer game data, Sterman found that the orders placed by most players conformed to APIOBPCS though, the parameters used by individual players were different.

Though APIOBPCS was used, variants other than the optimal parameters suggested by John et al. (1994) were also considered so as to simulate rogue seasonalities with different characteristics. Specifically, make to order (MTO) and make to stock (MTS) strategies or control systems are considered, because according to Buxey (1995) and Safizadeh and Ritzman (1997) these are more commonly found in industry than a mixture of MTO and MTS systems. Moreover, they are contrasts in terms of use of CONS or sales information (in the ordering decisions) and were therefore expected to yield different rogue seasonality characteristics. On the other hand, other researchers such as Anderson Jr et al. (2005) suggest that hybrid MTO-MTS strategies are more commonly used, and therefore it was also considered in the thesis.

Parameters within the hybrid MTO-MTS system were also varied. Two cases, Optimal and Unoptimal were considered, with these definitions being from a Bullwhip perspective. Optimal parameters are those which have been suggested by John et al (1994) for good dynamic behaviour, that is, low order amplification while Unoptimal parameters do not incorporate any pipeline feedback and are associated with greater order amplification (Sterman, 1989). Though the choice of Optimal parameters and Unoptimal parameters options are based on the Bullwhip effect, it was expected that these would generate rogue seasonality of different characteristics in the system as per the study by Kim and Springer (2008).

Other variants were applied to the simulation for assessing the robustness of the rogue seasonality findings. Delay or lead time was varied as it impacts the supply chain dynamics (Forrester, 1961; Chen et al., 2000). Similarly, the nature of demand affects the 
dynamics of the operating variables and was also therefore varied (Gilbert, 2005). Unlike most systems dynamics models, which are analysed by simulating them with a step or impulse function (Sterman, 2000; John et al, 1994; Towill et al., 2007), in this research most of the analysis is based on a stochastic stimulus (demand profile). This is because the simulation output is not just used for understanding rogue seasonality generation but more importantly is an input for developing and assessing rogue seasonality detection protocols (signature and index for measurement). The latter forms the thrust of this thesis and use of the more realistic stochastic demand profiles (Chopra and Meindl, 2001) in the same in comparison to step/impulse enhances the validity of the findings. Even otherwise, studies such as those by Dejonckheere et al. (2004) and Jaksic and Rusjan (2008) have based their analysis predominantly on stochastic demand profiles. Details of the stochastic demand pattern used in the thesis and the rationale for using them have been detailed in the respective chapters. Finally, batching of orders and shipments is a common practice and therefore sensitivity of the findings to the same was also assessed.

\subsubsection{Tools and techniques used}

There are two major tools for system dynamics simulation: specialist simulation packages such as Vensim $\bigotimes$, Stella® and iThink® and spreadsheet. Simulation packages provide ease in developing and analyzing complex models (graphical approach). However, getting the skills to make models in these packages is time consuming. Understanding the dynamics of individual variables and relating it to other variables is also less intuitive in these packages.

The alternative of spreadsheet simulation on the other hand offers a fast, easy way to build simulation models with maximum flexibility and learning. It involves entering the inputs and decisions into a spreadsheet and then relating them appropriately, by means of formulas, to obtain the outputs (Winston, 2005). Excel also has an in built Visual Basic Application (VBA) programming platform, which allows users to create their own computational functions as well to develop code/macros for automating routine tasks. VBA also allows use of controls such as scroll bar, spin button, option button, command button and check box to dynamically change simulation parameter values and related 
linked profiles of variables, thereby enabling better visualization and understanding of the dynamics. Add-ins such as for risk management (Crystal Ball囚) and data mining (XLSTAT囚) have further enhanced the capability of spreadsheets. The recent special issue of Interfaces focused on spreadsheet modeling is testimony to the relevance and usefulness of spreadsheets within the management community (LeBlanc and Grossman, 2008) which includes a discussion of Hewlett-Packard's experience in building and applying spreadsheet-based decision-support tools for forecasting, planning, procurement, and product management (Olavson and Fry, 2008). Similarly, Smith (2003) used three real world examples to show how spreadsheet modeling could be used for supply chain decision making.

The spreadsheet's flexibility in incorporating alternative model structures and parameters and the more intuitive understanding of dynamic behaviour that it provided were the key factors in the decision to use it for simulation. Another factor was that Excel links well with Matlab@ which was used for performing various time series transformations for rogue seasonality detection. The use of spreadsheet for simulation is well established and has been used in multiple contexts (John et al., 1994; Disney and Towill, 2003; Tang and Naim, 2004) and therefore provided confidence that it could be applied in the context of this thesis for rogue seasonality analysis. The final factor was that the author was already quite familiar with spreadsheets and VBA.

Spreadsheet simulation involved transferring the relevant difference equations (MTO/MTS and Beer game) into an Excel spreadsheet. However, changes such as in demand processes and parameters, lead time and lead time distributions also needed to be simulated and its impact on the behaviour of variables understood. Therefore, rather than making separate spreadsheets for each case, two generic simulators based on VBA were built: a generic APIOBPCS simulator and a Beer game simulator. These are depicted in Figures 3.3 and 3.4 below. The approach used in each case was the same as suggested by Smith (2003); a 'control panel' sheet consisting of different VBA controls (scroll bar, spin button, option button, command button and check box) and the actual data models on other worksheets. In the APIOBPCS simulator, the VBA controls are associated with different simulation variants such as control parameters $\left(T_{i}, T_{a}, T_{w}\right)$, delay/lead time $\left(T_{p}\right)$, 


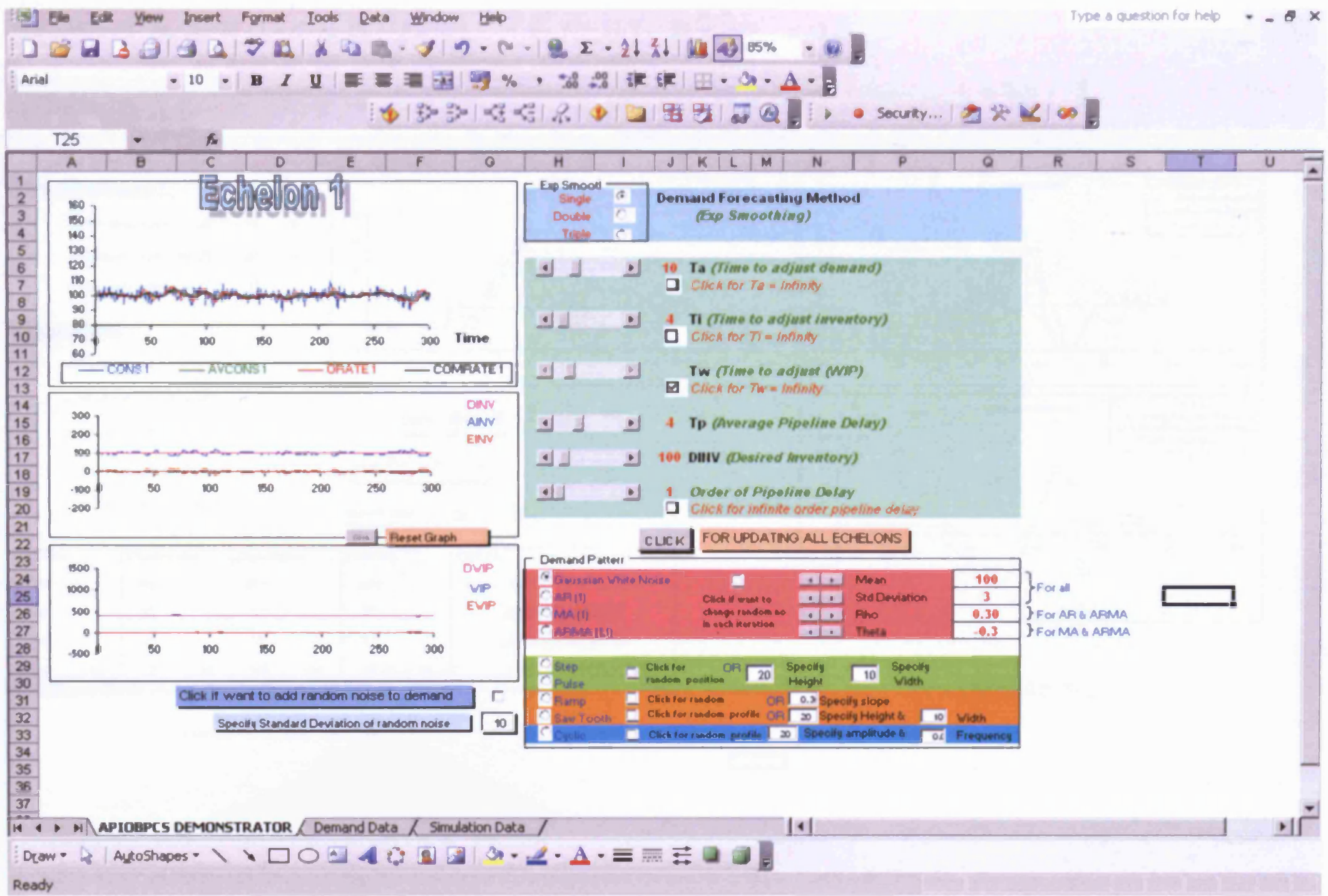

Figure 3.3. Screen shot of the VBA based APIOBPCS simulator used 


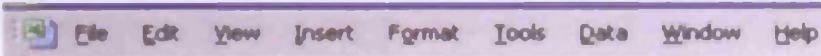

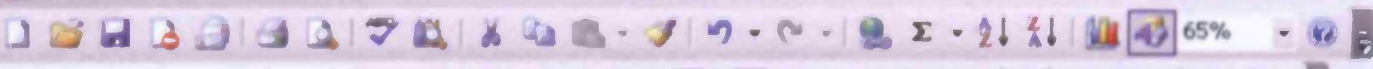

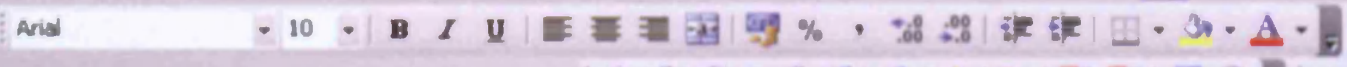

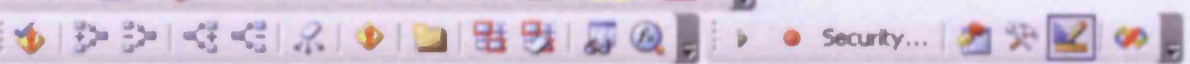

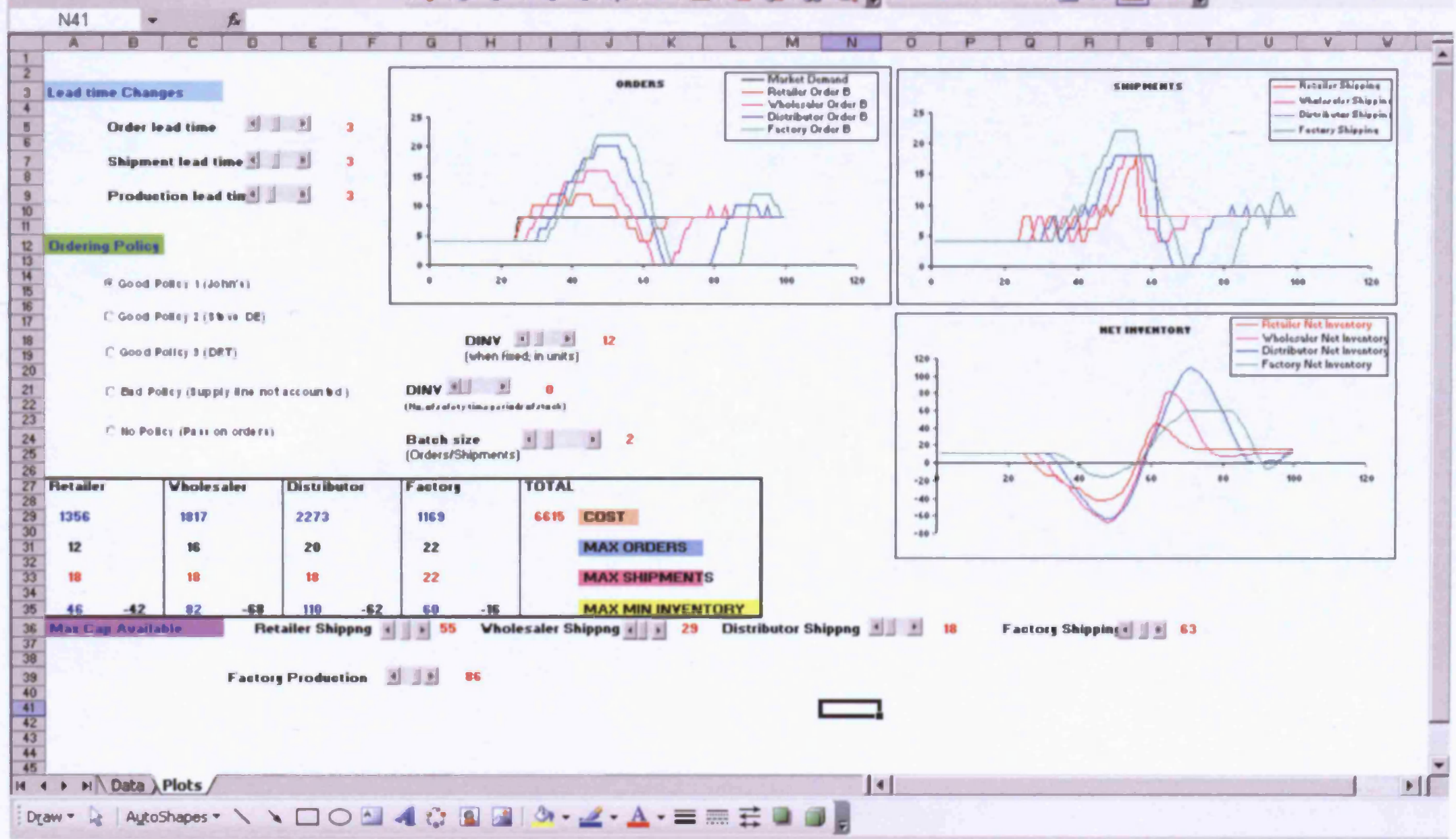

Figure 3.4. Screen shot of the VBA based Beer game simulator used 
lead time distributions (order of delay), demand processes and parameters and provide dynamic change capability. These simulation variants are linked to the simulation model, thereby effecting appropriate changes in the simulation data which in turn changes the linked plots of different variables on the 'control panel' page. Hence, this VBA enabled approach helps in relating changes in simulation variants to the dynamic profiles of different variables and therefore makes understanding of the dynamics easier. The three echelon APIOBPCS simulation was simulated by having three separate linked workbooks, with each workbook containing a similar single echelon APIOBPCS simulator. A similar approach as that used for the APIOBPCS simulator, was used to build the Beer game simulator.

While the simulator based approach was used for initial understanding of the rogue seasonality dynamics, it could not be used to generate the large volumes of contextual data required for a statistical assessment of the best time series techniques and features for rogue seasonality detection. A combined Excel, VBA and Matlab approach was used for the same.

An important part of the simulation process is the verification and validation of the simulation model (Law and Kelton, 2000; Sterman, 2000). Validation is the process of determining the accuracy of the simulation model compared to the system under study and is more appropriate for empirical simulation models, where there is a benchmark for comparison. Since, established generic models such as APIOBPCS and the Beer game were used in the thesis, the validation aspect was already covered. Verification on the other hand, is described as the process for checking the correctness of the translation from conceptual (causal loop) to a computer model, which in the case of the spreadsheet model means ensuring the correctness of the formulas and logic used in the model. The models were verified in different ways. Firstly, model outputs specifically oscillation frequency of the variables were compared to that suggested by control theory i.e. frequency response or amplitude ratio (FR or Amp R). Secondly, the exogenous demand variable was made constant across time, which, if the difference equations are correctly coded causes all variables to have a similar constant profile across time. Finally, similar conditions as that used in benchmark studies such as John et al. (1994) and Sterman 
(2000) were applied to the APIOBPCS and Beer game simulators respectively and the profiles and values of the model variables compared.

\subsubsection{Case studies}

Case studies are used in the thesis along with simulation because use of such multimethodology approach increases the validity of the findings. Case study is a preferred strategy in exploratory research because of its focus on contemporary phenomenon within a real life context and lack of investigator control (Yin, 1994). This explains the logic of research question 3. Decisions to be made when using the case study method are the nature and number of cases.

Case Selection: Case-based research can be expensive in terms of cost and time expended and therefore the nature of the case/s selected is an important part of the decision process. According to Eisenhardt (1989), the cases selected should be from an appropriate population in order to avoid as much as possible extraneous variations, while Stuart et al. (2002) suggests that case selection should consider the potential effects of industry, organization size, manufacturing processes and inter-organizational aspects. The choice of cases should follow theoretical rather than statistical reasons. Pettigrew (1990) proposes three criteria for case selection: (a) Extreme situations (b) Polar types as a way of disconfirming patterns from one case study to the other (c) High experience levels. He also describes as "planned opportunism" the practicalities of the process of choosing and gaining access to research sites.

The second important issue is whether single or multiple cases should be chosen and whether there is an optimum number for the latter. Eisenhardt (1989) advocates the use of four to ten cases, depending on the number of critical causal variables, in order to get proper results from case study research, but there are opponents to her approach who emphasize the importance of fewer cases, even single-case studies. Yin (1994) for example claims that a single case can be an appropriate research design when the case represents a unique or extreme case. Similarly, Stuart et al. (2002) claims to have conducted successful case research using as few as one to three companies. 
Appropriateness of the cases with respect to the research context therefore seems more important than the number of cases.

In the thesis two case studies from contrasting industries are used and provide different contexts for rogue seasonality analysis and detection. One study is from the steel industry characterized by stable demand profiles, while the other is from the grocery industry where the demand profiles are irregular and volatile. Monthly data was collected and analysed for the former while daily data was used for the latter. Both the case studies were analysed using predominantly an information centric approach focused on rogue seasonality.

\subsection{Time series transformations and features used for rogue seasonality detection}

As discussed earlier, this thesis has two parts: rogue seasonality generation and rogue seasonality detection. While the previous sections talked about rogue seasonality generation and related contextual data from simulation and case studies, this section discusses the time series transformation techniques and features used for rogue seasonality detection. The contextual rogue seasonality data (with different characteristics and intensities) consisting of time series of different variables are input into these techniques, and rogue seasonality detection is enabled based on appropriate signature and index. The relevance of signature based techniques and the inappropriateness of change point detection techniques for detection on a generic basis has been explained in the literature review chapter.

In signature based methods, signatures are usually derived on the basis of clustering or finding similarity relationships among the time series profiles of the operating variables. Time series clustering is predominantly based on the following three approaches (Liao, 2005): a) Raw data based (time series data used for clustering), b) Feature based (features extracted from the time series are used for clustering) and, c) Model based (time series is converted into a model and the model parameters are used for clustering). Clustering approaches based on time series data, though easier to interpret, have problems in computation (large, high dimensional data sets) as well as lower accuracy because of 
interference from noise. Feature based and model based approaches are therefore preferred (Liao, 2005; Keogh and Kasetty, 2003). Some of the commonly used features include amplitudes of Fourier transform (FT), wavelet coefficients of discrete wavelet transform (DWT), autocorrelation function (ACF) and cross correlation function (CCF) which is a feature of pairs of time series. The autoregressive (AR) model is the most commonly used model based approach. Details about these feature and model based approaches are given in the following paragraphs and summarized in Table 3.4.

a) Fourier transform (FT): This is an old technique involving decomposition of a time series into its constituent sinusoids at different frequencies (as per equation 3.1) which has been used to analyze periodic signals extensively (Chatfield, 2004). Periodicity in the data gets reflected as peak amplitude/s at corresponding frequency channel/s in the FT analysis.

$$
X_{f}=\frac{1}{\sqrt{n}} \sum_{i=1}^{n-1} x_{t} \exp \left(\frac{-2 \pi j f t}{n}\right)
$$

$\left|x_{,}\right|=$Amplitude of sinusoid of frequency $f ; \quad x_{1}=$ signal values at $t=0, \ldots . . n-1$

Frequency ()$=[0,1, \ldots n-1](1 / T), T=$ total sampling time, $j=\sqrt{-1}$

The utility of FT in time series clustering is, however, of recent origin with Agrawal et al. (1993) establishing the equivalence of similarity relationships between the time and frequency domains. This formed the basis of a fast and accurate method of clustering any time series (using fast Fourier transform) by using the amplitudes of the first few frequencies as features (non inclusion of high frequencies which are typically associated with noise ensures greater clustering accuracy). Variations of this technique have been tried in different studies such as Wu et al. (2000), Caiado et al. (2006) and Vlachos et al. (2006) as given in Table 3.4.

The FT based approach being suited for data with cyclicality, it was considered for rogue seasonality analysis by Thornhill and Naim (2006). However, their nature of application is different from the way FT based approach has been used in the thesis. While they used FT to eliminate lags between variable profiles for effective profile comparisons and subsequent manual clustering, in this thesis FT (using amplitudes from FT) has been 


\section{Table 3.4}

\section{Review of studies using different transformation techniques and features in time series clustering}

\begin{tabular}{|c|c|c|c|c|c|}
\hline & Reference & Transformation/Features used & $\begin{array}{l}\text { Comparison with } \\
\text { alternative methods }\end{array}$ & Clustering Technique & $\begin{array}{l}\text { Data used for } \\
\text { evaluation }\end{array}$ \\
\hline \multirow{7}{*}{$\begin{array}{l}\frac{1}{2} \\
\frac{1}{0} \\
\frac{0}{2} \\
\frac{\alpha}{2} \\
\frac{1}{2}\end{array}$} & $\begin{array}{l}\text { Tong and Dabas } \\
\text { (1990) }\end{array}$ & Residuals from ARIMA model & No & $\begin{array}{l}\text { Hierarchical clustering and MDS; } \\
\text { Euclidean dissimilarity measure }\end{array}$ & $\begin{array}{l}\text { Benchmark socio economic } \\
\text { dataset with seasonality }\end{array}$ \\
\hline & Picoolo (1990) & AR model parameters & No & $\begin{array}{l}\text { Hierarchical clustering and MDS; } \\
\text { Euclidean dissimilarity measure }\end{array}$ & $\begin{array}{l}\text { Economic time series with } \\
\text { seasonality }\end{array}$ \\
\hline & Deng et al. (1997) & ARMA model parameters & No & $\begin{array}{l}\text { Classification and query matching; } \\
\text { Euclidean dissimilarity measure }\end{array}$ & $\begin{array}{l}\text { Synthetic dataset with real } \\
\text { context }\end{array}$ \\
\hline & $\begin{array}{l}\text { Kalpakis et al. } \\
(2001)\end{array}$ & Cepstra of time series derived from AR model & Yes; FT, DWT, PCA, and ACF & $\begin{array}{l}\text { Partitioning around medoids; Euclidean } \\
\text { distance between cepstra as dissimilarity } \\
\text { measure }\end{array}$ & $\begin{array}{l}\text { Synthetic as well as } \\
\text { benchmark datasets }\end{array}$ \\
\hline & Maharaj (2000) & AR model parameters & No & $\begin{array}{l}\text { Hierarchical clustering based on p value } \\
\text { of a hypothesis test }\end{array}$ & $\begin{array}{l}\text { Synthetic as well as } \\
\text { economic dataset }\end{array}$ \\
\hline & $\begin{array}{l}\text { Xiong and Yeung } \\
(2002)\end{array}$ & ARMAAR model parameters & $\begin{array}{l}\text { Yes; Cepstra of time series derived } \\
\text { from AR model }\end{array}$ & $\begin{array}{l}\text { Expectation Maximisation (EM) } \\
\text { clustering }\end{array}$ & $\begin{array}{l}\text { Synthetic as well as } \\
\text { benchmark datasets }\end{array}$ \\
\hline & Ting et al. (2003) & AR model parameters & Yes; FT & $\begin{array}{l}\text { Classification and query matching: } \\
\text { Euclidean dissimilarity measure }\end{array}$ & $\begin{array}{l}\text { Synthetic as well as stock } \\
\text { price data sets }\end{array}$ \\
\hline \multirow{3}{*}{ 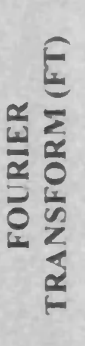 } & Wu et. al. (2000) & Amplitudes from FT & Yes; DWT (Haar) & $\begin{array}{l}\text { Classification and query matching; } \\
\text { Euclidean dissimilarity measure }\end{array}$ & Stock price datasets \\
\hline & Caiado et al. (2006) & Amplitudes from FT & $\begin{array}{l}\text { Yes; AR model, ACF, PACF, } \\
\text { IACF }\end{array}$ & $\begin{array}{l}\text { Hierarchical and k-means clustering; New } \\
\text { dissimilarity measure based on } \\
\text { periodogram }\end{array}$ & $\begin{array}{l}\text { Synthetic and economic } \\
\text { dataset }\end{array}$ \\
\hline & $\begin{array}{l}\text { Thornhill and Naim } \\
\text { (2006) }\end{array}$ & FT + PCA (principal component analysis) & No & Visual clustering by plotting & Supply chain dataset \\
\hline
\end{tabular}

MDS: Multidimensional Scaling SOM: Self organising map PCA: Principal Component analysis 
Table 3.4 Continued

\begin{tabular}{|c|c|c|c|c|c|}
\hline & Reference & Transformation/Features used & $\begin{array}{l}\text { Comparison with } \\
\text { alternative methods }\end{array}$ & Clustering Technique & $\begin{array}{l}\text { Data used for } \\
\text { evaluation }\end{array}$ \\
\hline \multirow{2}{*}{ 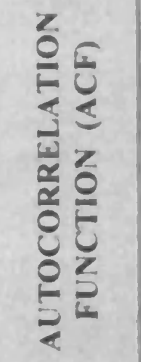 } & $\begin{array}{l}\text { Wang and Wang } \\
(2000)\end{array}$ & $\begin{array}{l}\text { Shape, trend, cyclic components, ACF and } \\
\text { Partial ACF }\end{array}$ & $\begin{array}{l}\text { Yes; Wavelet transformation of } \\
\text { time series (linear B-spline wavelet } \\
\text { functions) }\end{array}$ & $\begin{array}{l}\text { Classification and query matching: } \\
\text { Euclidean dissimilarity measure }\end{array}$ & $\begin{array}{l}\text { Synthetic as well as stock } \\
\text { price data set }\end{array}$ \\
\hline & Vlachos et al. (2006) & ACF with amplitudes from FT & $\begin{array}{l}\text { Yes; Cepstra of time series derived } \\
\text { from AR model }\end{array}$ & $\begin{array}{l}\text { Hierarchical clustering; New dissimilarity } \\
\text { measure based on periodogram }\end{array}$ & $\begin{array}{l}\text { Synthetic as well as } \\
\text { science, medicine and } \\
\text { manufacturing datasets }\end{array}$ \\
\hline \multirow{2}{*}{ 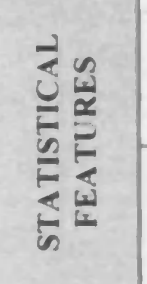 } & $\begin{array}{l}\text { McHutchon et. al. } \\
\text { (2005) }\end{array}$ & $\begin{array}{l}\text { Max, min, average, range, std deviation, root } \\
\text { mean square (rms), shape factor, crest factor, } \\
\text { impulse factor, kurtosis }\end{array}$ & $\begin{array}{l}\text { Yes; DWT (Daubechies } 4^{\text {th }} \text { order) } \\
\text { with statistical features }\end{array}$ & Visual clustering by plotting & $\begin{array}{l}\text { Synthetic dataset with real } \\
\text { maintenance condition } \\
\text { monitoring context }\end{array}$ \\
\hline & Wang et al. (2006) & $\begin{array}{l}\text { Trend, seasonality, periodicity, autocorrelation, } \\
\text { skewness, kurtosis, chaos, nonlinearity and self- } \\
\text { similarity }\end{array}$ & Yes; ACF, HMM, SAA & $\begin{array}{l}\text { Hierarchical clustering and SOM; } \\
\text { Euclidean dissimilarity measure }\end{array}$ & $\begin{array}{l}\text { Synthetic as well as } \\
\text { benchmark datasets }\end{array}$ \\
\hline \multirow{4}{*}{ 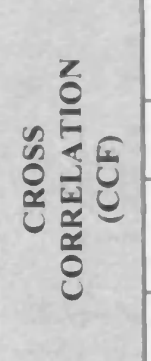 } & Bohte et al. (1980) & Cross correlation between time series & No & Own method/program & $\begin{array}{l}\text { Socio oconomic time } \\
\text { series }\end{array}$ \\
\hline & Goutte et al. (1999) & Cross correlation between time series & No & Hierarchical and $k$-means clustering & Biomedical dataset \\
\hline & Baragona (2001) & $\begin{array}{l}\text { Cross correlation between residual series } \\
\text { generated from fitting AR models }\end{array}$ & No & $\begin{array}{l}\text { Metaheuristic methods - Simulated } \\
\text { annealing, tabu search, genetic algorithm }\end{array}$ & Synthetic datasets \\
\hline & Aono et al. (2006) & Cross correlation between time series & No & Classification and query matching & Biomedical dataset \\
\hline \multirow{3}{*}{ 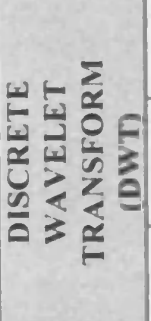 } & Chan and Fu (1999) & DWT (Haar) coefficients & Yes; FT & $\begin{array}{l}\text { Classification and query matching; } \\
\text { Euclidean dissimilarity measure }\end{array}$ & $\begin{array}{l}\text { Synthetic as well as stock } \\
\text { price data sets }\end{array}$ \\
\hline & Lin et al. (2004) & DWT (Haar) coefficients & Yes; FT & $\begin{array}{l}\text { k-means and its variant; Euclidean } \\
\text { dissimilarity measure }\end{array}$ & Benchmark datasets \\
\hline & Zhang et al. (2005) & DWT (Haar) coefficients & No & $\begin{array}{l}\text { Classification and query matching; } \\
\text { Euclidean dissimilarity measure }\end{array}$ & Benchmark datasets \\
\hline
\end{tabular}


directly used for automatic clustering. Despite its advantages, the FT based analysis has some deficiencies such as poor resolution of frequencies (especially for short series) and inefficient representation of non stationary/irregular profiles. Also, operating in the frequency domain lessens the intuitive feel of the results. Use of alternative time series techniques and features was therefore considered.

b) Discrete wavelet transform (DWT): Fourier Transform (FT), though a popular approach in time series clustering applications, is less efficient for non stationary/irregular signal profiles. Localised variations in such signals require more frequency channels for accurate representation because sinusoids represent global rather than local contributions to data. Wavelets are able to represent such signals more sparsely and accurately because:

a) They have an oscillatory profile of limited duration which is localised in both time as well as frequency domains

b) Their irregular and asymmetric profile can better represent discontinuities

c) Availability of wavelets with different characteristics such as shape and span (Daubecheis, Coiflets) can better fit different signal profiles

Figure 3.5 depicts a few commonly used wavelet profiles. An excellent coverage of time series analysis using wavelets is given in Percival and Walden (2000).
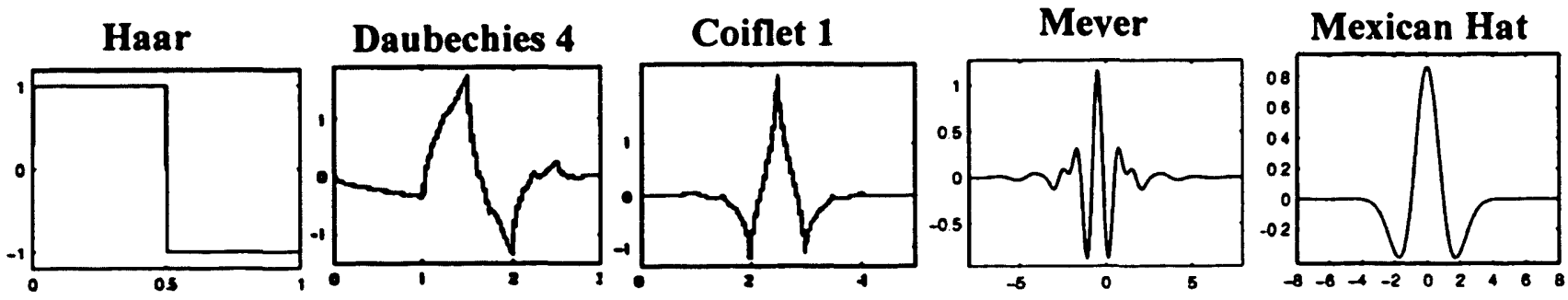

Figure 3.5 Profiles of some commonly used wavelets (Percival and Walden, 2000)

Wavelet based time series clustering applications involve use of discrete wavelet transformation (DWT) to compute wavelet coefficients which are then used as features in clustering. The coefficients represent the correlation between the original signal and the local oscillation represented by the wavelet basis function. The basis functions in turn are generated by time translation and frequency scaling of the analysing (mother or prototype) wavelet and can be mathematically represented as follows: 
Basis function $\Psi_{, . k}(t)=2^{J^{\prime}}{ }^{2} \Psi\left(2^{j} t-k\right)$ where $\Psi$ is the mother wavelet function $z^{j}$ is the scaling of $t$ and $2^{-j} k$ is the translation in $t$. It is to be noted that only dyadic (power of 2) time translations and frequency scaling is used in order to balance the competing requirements of accuracy and computational efficiency.

Any real function can be represented in terms of wavelet coefficients as given below:

Any real function $f(t)=\sum_{j, k} c_{j, k} \Psi_{j, k}(t) ; c_{j, k}=\left\langle\Psi_{j, k}(t), f(t)\right\rangle$ the wavelet coefficients of the DWT.

Operationally, the relevant decision parameters for this transformation are 1) Choice of mother wavelet, and 2) Level of decomposition of the signal.

In terms of choice of mother wavelet, the Haar wavelet was used as it is easy to comprehend and has been extensively used in time series clustering applications ( $\mathrm{Li}$ et al., 2002) which is also evident from Table 3.4. It can be mathematically represented as

$\Psi_{\text {Hoar }}(t)=\left\{\begin{array}{l}1, \text { if } 0<t<0.5 \\ -1, \text { if } 0.5<t<1 \\ 0, \text { otherwise }\end{array}\right.$

The mechanism of wavelet decomposition of the signal and the use of wavelet coefficients for clustering proceeds as follows: Decomposition of the signal proceeds hierarchically, starting with the highest frequency and gradually moving to lower frequencies as the decomposition level is increased. A time sequence of length $\mathrm{N}$ (which is required to be of power two can be broken down to any level upto $\log _{2} N$ with the wavelet coefficients at any level represented by $\left\{A_{j}, D_{j}, \ldots \ldots . D_{2}, D_{l}\right\}$. The $D_{j}$ 's are the detail coefficients representing the high frequency part of the signal with reducing frequency as we move from $D_{l}$ to $D_{j}$ to while the lowest frequency part of the signal is represented by $A_{j}$ or the approximation part. The broad trend of the time series is preserved in the approximation part whereas the localised changes are kept in the detail part. Clustering is done using only the approximation part of the sequence $A_{j}$ while the $D_{j}$ 's are discarded.

In terms of comparing DWT with DFT, a few studies have done so, with some finding it superior to DFT (Kahveci and Singh, 2001) and others finding them equivalent (Wu et 
al., 2000). However, no study has used discrete wavelet transform for data with cyclical features as used in this thesis.

c) Autocorrelation function (ACF): Autocorrelation function (ACF) of a time series reveals its time delayed correlation, that is, correlation between values of a time series across different time intervals or lags as given in equation 3.2. ACF for each time series (up to specified lags) are used as features for similarity assessment and clustering. Features in this method are therefore extracted from the time domain itself.

$$
A C F_{k}=\frac{\sum_{i=1}^{N-k}\left(x_{i}-\mu\right)\left(x_{i+k}-\mu\right)}{\sum_{i=1}^{N}\left(x_{i}-\mu\right)^{2}}
$$

$\mu=$ mean of data series, $k=$ lag, $N=$ number of data points

This method is suited for rogue seasonality detection as ACF of a cyclical data series oscillates with the same period as in the time domain. However, studies based on use of this method are limited. Wang and Wang (2000) used the Euclidean distance between ACFs to discriminate between two synthetically generated time series. Caiado et al. (2006) used Euclidean distance between ACF, in comparison with other techniques, to cluster and discriminate between stationary and non stationary synthetic time series and obtained around $75 \%$ clustering accuracy. Vlachos et al. (2006) used ACF along with FT in the context of periodicity detection and extraction of important periodic features. However, their focus of application was on individual time series. No study has used ACF in the context of clustering multiple data series with multiple cyclical characteristics as considered in the thesis.

d) Cross correlation function (CCF): Cross correlation function (CCF) defines the correlation between pairs of time series across various lags as per equation 3.3.

$$
C C F_{k}=\frac{\sum_{i=1}^{N-k}\left(x_{1}-\mu_{x}\right)\left(y_{1+k}-\mu_{y}\right)}{\sqrt{\sum_{i=1}^{N}\left(x_{1}-\mu_{x}\right)^{2}} \sqrt{\sum_{i=1}^{N}\left(y_{1}-\mu_{y}\right)^{2}}}
$$

$\mu_{x}$ and $\mu_{y}$ are the means of the two data series

$k=\operatorname{lag}$ and $N=$ number of data points 
This CCF based method has also seen limited applications in time series clustering studies. Bohte et al. (1980) used the cross correlation similarity measure for clustering a socio-economic time series. Baragona (2001) used cross correlation between residuals generated from fitting high order autoregressive (AR) models to the time series as the similarity measure in clustering. Clustering was done using metaheuristic techniques and synthetic time series were used for the evaluation. Goutte et al. (1999) used the cross correlation similarity measure and hierarchical clustering for clustering time series in neuro imaging while Aono et al. (2006) used the same similarity measure for pattern recognition and classification of bio-signals. The CCF based method has not been used for data with cyclicality, though like ACF, it operates in the time domain which makes interpretation of results from it easier.

e) Autoregressive model (AR): Model based methods have roots in statistical time series analysis. The basis of this approach is that any time series can be represented as a function of its values in the previous time periods (Box and Jenkins, 1976). The number of past time periods used is reflected in the order of the model, which is a decision parameter. Operationally, an AR model is fitted to each time series up to the specified order by minimizing the sum of square errors and using the respective model coefficients as features for clustering. Equation 4.4 gives an AR model of order $p$.

$$
X_{t}=\alpha_{1} X_{t-1}+\alpha_{2} X_{t-2}+\alpha_{3} X_{t-3}+\ldots \ldots \ldots . . . \alpha_{p} X_{t-p}+Z_{t}
$$

$p=$ model order, $\alpha_{l} \alpha_{p}: A R$ coefficients and $Z_{t} \sim$ random process

$E\left(Z_{1}\right)=0 \& \operatorname{Var}\left(Z_{\nu}\right)=\sigma_{z}^{2}$

Advantages in using the AR model approach include significant dimensionality reduction (improves computational efficiency) and ability to cluster time series of different lengths.

The AR model based approach and its variants have been used in various studies. Piccolo (1990) clustered fourteen economics related time series using the Euclidean distance between the AR coefficients and could discriminate them on the basis of their seasonal component. Ting et al. (2003) also used the Euclidean distance between the AR coefficients to cluster synthetic data with seasonality characteristics and found this 
method to be more accurate and efficient (in terms of number of parameters used) than FT. Maharaj (2000) used the $p$ value of the hypothesis test based on the differences between the AR coefficients as the dissimilarity measure to cluster synthetic as well as economic datasets. Kalpakis et al. (1999) used the Euclidean distance between cepstra calculated from the AR model of each time series for clustering. Some researchers such as Tong and Dabas (1990) and Deng et al. (1997) have used ARIMA rather than AR model representations for the time series. ARIMA model representations have MA (moving average part) and I (a part reflecting trend in the data) beside the AR term/s and are an equivalent but more compact representation of a time series than pure autoregressive (AR) but less intuitive and computationally slower.

f) Statistical features: This method like the model based approach seeks to overcome difficulties in clustering long time data series (high dimensionality), data series with missing data or data whose lengths are unequal. Instead of a point to point distance metric, it uses the statistical features of the data sets for clustering. For example, Wang et al. (2006) use trend, seasonality, periodicity, autocorrelation, skewness, kurtosis, chaos, nonlinearity and self-similarity as features and the Euclidean distance for clustering. They showed this method to be effective for synthetic and benchmark data sets. McHutchon et al. (2005) applied other statistical features such as $\max , \min$, average, range, standard deviation, root mean square (rms), shape factor, crest factor, impulse factor, kurtosis and showed their utility in discriminating faults in a remote condition monitoring context. The use of statistical features for time series clustering is not sufficiently matured: there are limited studies on this subject and the best/most appropriate features to be used for different contexts have not been established. Moreover, use of aggregate statistical measures for profiles in this method reduces intuitiveness/explanation of the results obtained.

In summary, each technique discussed above has some relative advantages over other techniques. However, Table 3.4 highlights that few studies have compared the performance of their techniques and features with other rival techniques, and those that have, have done so with one or two techniques (Keogh and Kasetty, 2003; Liao, 2005). Secondly, the data that these studies have used to establish the superiority of a particular 
technique has a bias (Keogh and Kasetty, 2003). Primarily synthetic, non contextual data is used in most of the studies, with a few supplementing it with empirical data but that too on stock price or the finance domain as evident in Table 3.4. Since the results and effectiveness of different techniques are domain specific, and none of these studies has used any supply chain related data, their applicability in the supply chain context is limited. In this thesis, therefore, all the time series transformation techniques except statistical features have been applied and their effectiveness compared. Moreover, the data used for this assessment (from simulation and case studies) have a supply chain context. Statistical features were not used as they are not matured for application.

Different number of features were considered for each transformation technique to assess sensitivity of the results to the same. For example, for Fourier transform (FT), the feature options considered were FT 7 (amplitudes of first seven frequencies), FT 28 (amplitudes of first twenty eight frequencies), FT Total (amplitudes of all frequencies) and FT $80 \%$ energy (as many frequencies that have $80 \%$ of the total energy) for both linear and non linear simulation data analysis. For other transformations also, different numbers of features were similarly considered. The number of features used are discussed in detail in Chapter 4. For the case study analysis, the number of features used are different from simulation, due to data availability issues with the former. All time series transformations in the thesis were computed using Matlab® (see Appendix D).

\subsection{Clustering and rogue seasonality signature}

Profiles of different variables, either in the time or transformed domain, are required to be analysed to determine the characteristic signature for rogue seasonality. Though any of the three commonly used techniques of Classification, Association and Clustering could be applied for this analysis (these techniques are explained in Section 2.4.3.2), clustering was preferred because it is the most commonly used technique in time series applications (Liao, 2005). Moreover, for rogue seasonality, intial insights obtained from control theory analysis suggested clustering to be more appropriate for signature determination as discussed in the next chapter. 
Clustering techniques are concerned with partitioning of data sets (profiles of variables in time/transformed domain in this case) into a small number of homogenous groups or clusters with a characteristic profile of clusters associated with rogue seasonality deemed to be the latter's signature. Amongst the many clustering methods, two of the most popular ones are k-means and hierarchical (Tan et al., 2005). The k-means method is an iterative process that searches for division of data objects into $(k)$ clusters to minimize the sum of Euclidean distances between each object and its closest cluster centre. Though it is a robust and scalable method, it is sensitive to the initial selection of cluster centres and requires the number of clusters $(k)$ to be specified before clustering. The hierarchical clustering method on the other hand does not require a priori specification of the number of clusters and yields good visualisation of cluster formation (Everitt, 1993). Both these attributes are important in view of the exploratory nature of the rogue seasonality signature subject, and therefore hierarchical clustering was used. In hierarchical clustering, each point (time series represented as a point in multidimensional space) is merged into clusters based on their relative closeness or similarity relationships. The clusters formed are again clustered on the same basis and this process is repeated until finally there is one all encompassing cluster. Hierarchical clustering is graphically displayed as a tree like diagram called the dendogram which gives the order in which the cluster-sub-cluster relationships formed at different stages. Clustering of data in the thesis was done using the well known XLSTAT® software which directly takes inputs from the Excel spreadsheet.

Use of hierarchical clustering requires three decision choices: a) Similarity measure, b) Basis for merging two clusters and, c) Where to cut the dendogram to determine the number of clusters. The dissimilarity measure used for all except the $\mathrm{CCF}$ method was the Euclidean distance as it is commonly used in time series clustering (Agrawal et. al., 1993; Liao, 2005) and gives results of comparable accuracy vis-à-vis various other similarity measures (Keogh and Kasetty, 2003). It works well with all the time series techniques considered and is also fairly intuitive and easy to implement. It is defined as follows: 


$$
d E=\sqrt{\sum_{k=1}^{p}\left(x_{i k}-y_{j k}\right)^{2}}
$$

$d_{E}=$ Euclidean distance

$x_{i}$ and $y_{j}$ are $p$ dimensional vectors

For CCF, two measures of similarity have been used in the literature. One is a composite similarity measure based on all the cross correlation values at different lags (Bohte et al., 1980) while the other one uses the maximum cross correlation value within the specified maximum lags (Baragona, 2001) as the similarity measure. We used the method by Baragona because of its intuitive simplicity.

For merging two clusters, Ward's algorithm, a popular method was used (Halkidi et. al., 2001). This forms a cluster hierarchy by merging clusters that result in the smallest increase in the value of the sum-of-squares variance. At each clustering step, all possible mergers of two clusters are tried, the sum-of-squares variance computed for each and the one with the smallest value is selected. As this algorithm works only with Euclidean distance dissimilarity, it is not suited for the CCF method. Hence, complete linkage clustering was used for the CCF method instead, in which clusters are merged based on relative proximity or distance between them, with distance being the maximal object-toobject distance between the clusters.

Finally, the decision on where to cut the dendogram and identify clusters, depends on its tree structure and patterns of branches. For dendograms, in which branches are separated and join far apart, distinct clusters of the variables attached to the branches can be identified. However, for others, identifying clusters and hence pattern of relationships between the variables is subjective and difficult. This is further complicated by issues such as the scale used. Hence, an alternative approach based on direct use of dissimilarity values between the variables was considered for rogue seasonality detection.

\subsection{Index of rogue seasonality}

Identifying clusters and hence pattern of relationships between the variables is subjective and difficult. Hence, an alternative approach based on using an index derived from the dissimilarity values between the various supply chain operating variables was considered. 
This index was defined so as to objectively detect and assess rogue seasonality intensity in a generic sense. Subsequently, it was also used as a basis to evaluate the effectiveness of different time series techniques and features for rogue seasonality detection. Since, definition of the index emerged from the simulation results, it is best explained in the relevant section which is the next chapter.

\subsection{Conclusion}

This chapter has explained the philosophy, methodology and methods used to conduct the research and answer the research questions. The basis for adopting a positivistic stance and using quantitative methodologies was covered initially, followed by an explanation of the two main research threads: rogue seasonality analysis and related contextual data and rogue seasonality detection. The rationale of using a multi methodology, simulation and case study approach for rogue seasonality analysis was explained followed by details of the simulation process used: systems dynamics with control theory for better analytical support, APIOBPCS control system (John et al., 1994) and its variants such as make to order (MTO) and make to stock (MTS) in linear system and hybrid MTO-MTS in non linear system (Beer game) and spreadsheet (Excel), Visual Basic for applications (VBA) and Matlab as the tools of simulation. Details of the two case studies used, one from the steel industry and the other from the grocery industry were explained next. Finally, rogue seasonality detection in terms of signature of rogue seasonality and index of rogue seasonality were covered. This included the conceptualisation of the rogue seasonality signature by clustering features obtained from alternative time series transformations such as Fourier transformation (FT), auto correlation function (ACF), cross correlation function (CCF), autoregressive modelling (AR model) and discrete wavelet transform (DWT). The nature and application of each transformation technique in generic time series clustering applications as well as the appropriateness of using hierarchical clustering was explained. Finally, the relevance of the rogue seasonality index and its role in eliminating some of the deficiencies of rogue seasonality signature was briefly highlighted. 


\section{Chapter 4 - Analysis of rogue seasonality in a linear supply chain system}

This chapter analyses rogue seasonality in a simulated linear supply chain system. Continuous control theory (' $s$ ' domain) together with system dynamics simulation of make to order (MTO) and make to stock (MTS) systems is used in the analysis. First, a single echelon system is analysed using control theory (specifically frequency response or FR) to understand the dynamics of variables in rogue seasonality generation and a potential signature that could be used for its detection. These findings are then validated using system dynamics simulation. The signature is then quantified to define a numerical index as an indicator of the intensity of rogue seasonality. Subsequently, the effectiveness of the signature and index is assessed using a large scale three echelon simulation. This involved varying the magnitude and order of delays as well as exogenous demand processes and their parameters to generate rogue seasonalities of different characteristics and intensities in the system. Alternative time series transformations such as Fourier transform (FT), autocorrelation function (ACF), cross correlation function (CCF), discrete wavelet transform (DWT) and autoregressive (AR) model are then applied on the system variables and the rogue seasonality signature and index derived in each case, using their respective features. The different features are compared in terms of consistency in assessing rogue seasonality, ability to discriminate rogue seasonalities of different intensities and stochastic robustness, to determine the best feature for rogue seasonality detection.

\subsection{Rogue seasonality in single echelon systems}

A sequential modelling process from simple to complex structures enables better understanding of the dynamics of systems. A single echelon APIOBPCS is therefore initially used to understand the dynamics associated with rogue seasonality. Specifically, make to order (MTO) and make to stock (MTS) systems derived from the APIOBPCS archetype are used, with the rationale for the choice of these systems having been explained in Chapter 3. 
While the APIOBPCS based MTS system has been extensively studied (Mason-Jones and Towill, 1997; Disney and Towill, 2005), the study of the MTO system has been limited. Moreover, alternative information flow and feedback structures have been used to model the latter. For example, Naim et al. (2007) use APIOBPCS with parameter values $0, \infty$ and $\infty$ for $\mathrm{Ta}, \mathrm{Ti}$ and $\mathrm{Tw}$ respectively to model the MTO system; inventory and work in progress feedback are eliminated and quantity ordered is equivalent to demand. Lead time (Tp) is considered constant (i.e. independent of demand) in this approach, implying an infinite production/order processing capacity. A similar approach is used by Towill (2005). On the other hand, researchers such as Wikner et al. (2007) recognize the capacity to be limited in their modeling approach. All the demand placed on this system is not met and surplus orders are temporarily stored in an order book, information on which is used in the ordering policy to effectively manage capacity and lead time flexibilities. Similar approaches as Wikner et al. (2007) though with different terminologies have been proposed by Helo (2000) and Anderson Jr et al. (2005).

In this thesis, though either of the above two approaches could be used to model an MTO system, the approach of Naim et al. (2007) and Towill (2005) is actually used. This is because the focus here is on detection of rogue seasonality which required use of systems exhibiting contrasting rogue seasonality characteristics and intensities. Modeling an MTO system in the way suggested by Naim et al. (2007) ensures no rogue seasonality generation, which meant that it could be an effective baseline for devising rogue seasonality detection and measurement protocols.

For an MTS system, there was no such dilemma as for the MTO system. The parameters suggested by Naim et al. (2007), which are $\mathrm{Ta}=\mathrm{Tw}=\infty, \mathrm{Ti}=\mathrm{Tp}$ were used which were substituted into the APIOBPCS difference equations and transfer functions to derive them for an MTS system.

The MTO and MTS systems are analysed for different order of delays. Order of delay reflects the distribution of output around the average delay. In a system with order of delay one, the input is completely mixed and a proportion of the output starts exiting the system immediately after inputs into the system. This is analogous to chemical 
processing. On the other hand in an order of delay infinity system, input and output enter and exit the system after a fixed average delay and in the same sequence, as may be found in automotive assembly. Both order of delay one and infinity were used so as to not only make the models more representative (as observed in practice), but also to generate different rogue seasonality characteristics in the systems. Further details about order of delay are provided in Sterman (2000).

The transfer functions of variables for the MTO and MTS systems are given in Appendix B. A noticeable fact is that unlike in the generic APIOBPCS system (John et al., 1994), number of unique variables in these systems are fewer which is because many variables are equivalent to one other as evident in equations B.9, B.11, B.18, B.19, B.20 in Appendix B. The MTO and MTS systems are analysed using a combined control theorysystem dynamics simulation approach as proposed by Towill (1982). The benefits of such a combined approach have been discussed in the Methodology chapter (Section 3.6.1.3) and are therefore not repeated here.

\subsubsection{Control theory (frequency response) analysis}

Each single echelon MTO and MTS system is first analysed using the FR approach based on Towill and del Vecchio (1994), Dejonckheere et al. (2003) and Jaksic and Rusjan (2008) which involves representing the profile of variables in the frequency domain. This approach is suited for data with cyclical characteristics (Chatfield, 2004), and was therefore considered for the analysis of rogue seasonality. In this approach, the transfer function of each variable of interest is first converted from the ' $s$ ', (continuous) to the frequency (' $\omega$ ') domain by substituting (' $j \omega$ ) in place of ' $s$ ' and determining the absolute value of the function. This function called FR gives the ratio of amplitude of the sinusoidal output to sinusoidal input at various frequencies $(\omega)$ and is therefore also referred to as amplitude response (Amp R). Since every time series consists of and can be broken down into its constituent sinusoids at different frequencies (each with different amplitudes), the FR profile provides information about the frequencies in the input time series that would be amplified (corresponding to FR $>1$ ), attenuated (corresponding to FR $<1$ ) and for whom there would be no change (corresponding to $F R=1$ ). 
Based on the above approach, FR as a function of frequency $(\omega)$ is derived for each unique variable in each analysed system, specifically MTO and MTS with order of delay one and infinity. Here unique means that the other system variables are either equivalent to or are a multiple of these variables and therefore equivalent post-amplitude scaling, which is a typical step used in profile comparisons. The FR profiles are then analysed at critical frequencies such as at zero frequency $\left(\omega_{0}\right)$, frequency at which FR is maximum $(\omega \max F R)$, frequency at which crossover from amplification to attenuation takes place,

that is, $F R=1$ ( $\left.\omega_{\text {crossover }}\right)$ and very high frequency $\left(\omega_{\infty} ; \infty\right.$ is used as surrogate for very high). This is to understand the frequency characteristics of the system, get insights about rogue seasonality and a potential signature that could be associated with its presence. A summary of the analysis is given in Table 4.1. Matlab® symbolic toolbox was used for performing the relevant computations.

\subsubsection{Frequency response (FR) analysis of a MTO system}

The summary of the FR analysis given in Table 4.1 highlights the fact that each system has only a few unique variables. Secondly, FR for each variable is computed relative to exogenous demand (CONS).

The FR profiles of unique variables in the MTO systems, both delay order one as well as infinity have a maximum value of either one or $T_{p}$. Those which have a maximum value of one would not amplify any frequency in the CONS input. For example, the maximum FR value of COMRATE/CONS for delay order one system is one, which means that the COMRATE would not amplify any frequency in CONS. Other variables which have a maximum FR value of $T_{p}$ such as $E W I P / C O N S$ for delay order one and infinity and EINV/CONS for delay order infinity have FR profiles which are either continuously increasing or decreasing. Amplitude scaling of these profiles with respect to $T_{p}$ (typically applied before comparing profiles) means no amplification of any frequency in CONS by EWIP and EINV variables either. Therefore, with none of the variables in an MTO system amplifying any frequency in CONS, no seasonality distinct from CONS (referred to as rogue seasonality in the subsequent sections) is generated by the system. 
TABLE 4.1 - Frequency response (FR) functions of variables in make to order (MTO) and make to stock (MTS) systems

Outputs are given here in terms of angular frequencies ( $\omega$

\begin{tabular}{|c|c|c|c|c|c|c|c|}
\hline & Frequency response (FR) function of unique variables & $\begin{array}{l}\text { Profile of FR } \\
\text { function }\end{array}$ & $\begin{array}{l}\text { FR at freq } \\
(\omega)=0 \text { or } \omega_{0}\end{array}$ & $\begin{array}{l}\text { Freq }(\omega) \text { for } \\
\max F R\end{array}$ & Max FR & $\begin{array}{l}\text { Freq ( }() \\
\text { at } F R=1 \text { or } \\
\omega_{\text {crossover }}\end{array}$ & $\begin{array}{l}\text { FR at freq } \\
(\omega)=\infty \text { or } \\
\omega_{\infty}\end{array}$ \\
\hline \multirow{2}{*}{ 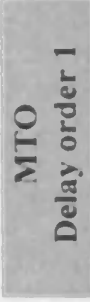 } & $\frac{\text { COMRATE }}{\text { CONS }}=\frac{1}{\sqrt{1+T_{p}^{2} \omega^{2}}}$ & & 1 & 0 & 1 & NA & 0 \\
\hline & $\frac{E W I P}{\operatorname{CONS}}=\frac{T_{p}^{2}}{\sqrt{1+T_{p}^{2} \omega^{2}}}$ & $\omega \rightarrow$ & 0 & $\propto$ & $T_{p}$ & NA & $T_{p}$ \\
\hline \multirow{3}{*}{ 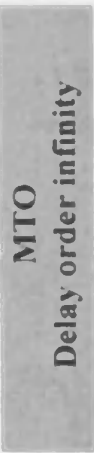 } & $\frac{\text { COMRATE }}{\text { CONS }}=\frac{\sqrt{\left(T_{p}^{4} \omega^{4}-60 T_{p}^{2}{ }^{2}+144\right)^{2}+\left(12 T_{p}^{3} \omega^{3}-144 T_{p} \omega\right)^{2}}}{\left(12-T_{p}^{2} \omega^{2}\right)^{2}+36 T_{p}^{2} \omega^{2}}$ & & 1 & all & 1 & NA & 1 \\
\hline & $\frac{E I N V}{C O N S}=-\frac{\sqrt{\left(12 T_{p}^{3} \omega^{2}-144 T_{p}\right)^{2}+\left(72 T_{p}^{2} \omega\right)^{2}}}{\left(12-T_{p}^{2} \omega^{2}\right)^{2}+36 T_{p}^{2} \omega^{2}}$ & & $T_{p}$ & 0 & $T_{p}$ & NA & 0 \\
\hline & $\frac{E W I P}{C O N S}=-\frac{\sqrt{\left(T_{p}^{5} \omega^{4}+24 T_{p}^{3} \omega^{2}\right)^{2}+\left(72 T_{p}^{2} \omega\right)^{2}}}{\left(12-T_{p}^{2} \omega^{2}\right)^{2}+36 T_{p}^{2} \omega^{2}}$ & & 0 & $\propto$ & $T_{p}$ & NA & $T_{p}$ \\
\hline \multirow{2}{*}{ 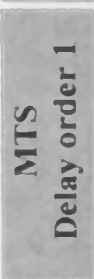 } & $\frac{\text { ORATE }}{\operatorname{CONS}}=\frac{\sqrt{1+T_{p}^{6} \omega^{6}}}{\left(1-T_{p}^{2} \omega^{2}\right)^{2}+T_{p}^{2} \omega^{2}}$ & & 1 & $\frac{0.856}{T_{p}}$ & $\begin{array}{l}1.468 \\
\text { (constant) }\end{array}$ & $\frac{1.414}{T_{p}}$ & 0 \\
\hline & $\frac{\text { COMRATE }}{\text { CONS }}=\frac{1}{\sqrt{\left(1-T_{p}^{2} \omega^{2}\right)^{2}+T_{p}^{2} \omega^{2}}}$ & & 1 & $\frac{0.707}{T_{p}}$ & $\begin{array}{l}1.155 \\
\text { (constant) }\end{array}$ & $\frac{1}{T_{p}}$ & 0 \\
\hline \multirow{3}{*}{ 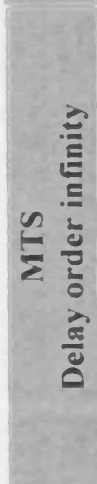 } & $\frac{\text { ORATE }}{\operatorname{CONS}}=\frac{\sqrt{\left(T_{p}^{4} \omega^{4}-60 T_{p}^{2} \omega^{2}+144\right)^{2}+\left(T_{p}^{5} \omega^{5}+24 T_{p}^{3} \omega^{3}\right)^{2}}}{\left(12-7 T_{p}^{2} \omega^{2}\right)^{2}+\left(6 T_{p} \omega-T_{p}^{3} \omega^{3}\right)^{2}}$ & & 1 & $\frac{1.306}{T_{p}}$ & $\begin{array}{l}2.307 \\
\text { (constant) }\end{array}$ & $\frac{1.906}{T_{p}}$ & 0 \\
\hline & $\frac{\text { COMRATE }}{\text { CONS }}=\frac{\sqrt{\left(13 T_{p}^{4} \omega^{4}-132 T_{p}^{2} \omega^{2}+144\right)^{2}+\left(60 T_{p}^{3} \omega^{3}-T_{p}^{5} \omega^{5}-144 T_{p} \omega\right)^{2}}}{T_{p}^{6} \omega^{6}+37 T_{p}^{4} \omega^{4}-132 T_{p}^{2} \omega^{2}+144}$ & & 1 & $\frac{1.306}{T_{p}}$ & $\begin{array}{l}2.307 \\
\text { (constant) }\end{array}$ & $\frac{1.906}{T_{p}}$ & 0 \\
\hline & $\frac{W I P}{\operatorname{CONS}}=\frac{12 T_{p}}{\sqrt{\left(12-7 T_{p}^{2} \omega^{2}\right)^{2}+\left(6 T_{p} \omega-T_{p}^{3} \omega^{3}\right)^{2}}}$ & & $T_{p}$ & $\frac{1.293}{T_{p}}$ & $2.141^{*} T_{p}$ & $\begin{array}{l}\text { Complex } \\
\text { function of } T_{p}\end{array}$ & 0 \\
\hline
\end{tabular}




\subsubsection{Frequency response (FR) analysis of a MTS system}

In contrast to MTO systems, all unique variables in MTS systems have FR profiles which are increasing-decreasing and with an intermediate maxima. Although such profiles have been discussed in the past by Towill and del Vecchio (1994), it was in the context of IOBPCS, not an MTS system as considered here, and only for a single variable (order or ORATE). Moreover, their nature of analysis was graphical and not analytical as done here. Dejonckheere et al. (2003) have also similarly discussed the FR profile of only the order variable in their analysis and for an order up to system (which can be used to represent APIOBPCS). Their FR analysis was also not comprehensive and restricted to select parameter (ordering) combinations. Finally, Jaksic and Rusjan (2008), who used Dejonckheere et al.'s approach but with a different replenishment rule, also had a similar issue with regard to their nature of analysis. These three studies also differ from the present study in terms of the frequency range that is used in the analysis. While the former use FR profile information across the entire frequency range (to understand the Bullwhip effect and determine its magnitude called noise bandwidth), rogue seasonality analysis and assessment is based on only the amplification range frequency (i.e. frequencies for which FR $>1$ ). This will be clearer in the next paragraph.

The FR profiles of all variables in the MTS system are greater than one in the frequency

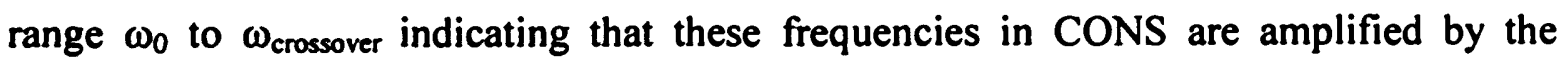
system variables. This endogenous amplification of frequencies, independent of the frequency characteristics of CONS, is referred to as rogue seasonality generation in the system. A feature of rogue seasonality generation, and which is evident in each of MTS delay order one and infinity systems, is that the FR profiles of the variables in the system are similar to each other. Since every CONS can be broken down into its constituent sinusoids and FR for a variable represents the extent to which it amplifies each of these sinusoids, similarity among FR profiles of variables means that all these variables would show a similar oscillatory time series profile which would be different from the CONS profile. However, since a range of frequencies from $\omega_{0}$ to $\omega_{\text {crossover }}$ are amplified, each with a different degree of amplification (as represented by the FR), the time series 
profiles of variables would show a mixture of cycles although they would have a dominant cycle at frequency $\omega_{\max }$ FR.

Another observation with regard to MTS systems is that the maximum FR value (maximum amplification) is independent of lead time $\left(T_{p}\right)$ for all variables (except WIP/CONS in delay order infinity system) and corresponds to a frequency which is inversely related to $T_{p}$. This means that an increase/decrease in $T_{p}$ would reduce/increase the time period (or frequency) of oscillation of the profiles without having any impact on their amplitudes. This observation seems to be at variance with Kim and Springer (2008), who suggest that increasing/decreasing lead time $\left(T_{p}\right)$ generates strong/weak cyclicality. However, the strength of seasonality definitions used in the two cases are different and therefore the results are not exactly comparable. Kim and Springer use a subjective definition, albeit one with a frequency bias; endogenous seasonality in a profile is strong if it showed multiple oscillations before reaching equilibrium and weak if it did so once or less. On the other hand the definition used in this thesis is based on the intensity or amplitude of rogue seasonality. Observation of maximum FR being independent of lead time $\left(T_{p}\right)$ also seems to contradict the direct relationship between $T_{p}$ and Bullwhip (increase/decrease in $T_{p}$ leads to increase/decrease in Bullwhip) pointed out by many researchers (Lee et al., 1997a; Towill and McCullen (1999). However, here also there is no contradiction as Bullwhip is based on the square of the FR profile across the whole frequency range (the measure of Bullwhip effect called "noise bandwidth" suggested by Dejonckheere et al. (2003) is based on the area under the squared FR profile) while the rogue seasonality analysis and findings are based on the FR profile and that too in a limited frequency range where $F R>1$.

\subsubsection{Evolution of rogue seasonality signature}

The MTS and MTO systems discussed above, in which variables exhibit, do not exhibit rogue seasonality provide effective discrimination, and therefore, a basis for developing the signature for the same. In the MTS system, the FR profiles of most of the unique variables and hence most available variables are similar. At the same time, because these variables amplify frequencies between $\omega_{0}$ and $\omega_{\text {crossover }}$ in CONS, they would be 
dissimilar from the latter. With similarity of FR translating into similarity in profiles in the frequency domain, this characteristic presentation of profiles (many variables are similar to each other and dissimilar from CONS) could be used as a signature to indicate the presence of rogue seasonality in a new case. In contrast to this, in a system without rogue seasonality such as MTO, the profiles of variables do not show amplification at any frequency and hence would not be dissimilar to CONS.

While the signature for rogue seasonality could be defined at a conceptual level as above, it needs to be shown and verified using time series data from these MTO and MTS systems. This is required because of the following reasons:

a) Even though the FR profiles of different variables in MTS systems are similar, which has been identified to be an indicator of rogue seasonality, the similarity is not exact as can be seen in Table 4.1. For example, in the MTS delay order one system, $\omega_{\max }$ FR, $\omega_{\text {crossover }}$ and maximum FR values for ORATE/CONS and COMRATE/CONS are marginally different. The same is true for other variables such as ORATE/CONS and WIP/CONS in the MTS delay order infinity system. Simulation is required to assess if these marginal dissimilarities affect the conceptual logic of the rogue seasonality signature.

b) Another factor which affects the alignment of variables and could therefore impact the effectiveness of the signature is that multiple frequencies between $\omega_{0}$ and $\omega_{\text {crossover }}$ are amplified, not just one frequency.

c) Finally, the earlier assumption about amplitude scaling (with $T_{p}$ ), of variables having a maximum FR value of $T_{p}$ in MTO systems needs to be assessed, Also, there is a need to apply all, rather than only unique variables and show them to be effective in formulating the rogue seasonality signature.

Single echelon MTO and MTS systems are therefore simulated and the time series profiles of variables generated from the simulation are used to determine the rogue seasonality signature. 


\subsubsection{Simulation output analysis}

Details of the simulation are provided in the Methodology chapter (Sections 3.6.1.4 and 3.6.1.5). Each system was excited with Gaussian CONS and the time series profiles of the system variables were analyzed. These profiles are also transformed into the frequency domain using Fourier transform (FT) and their spectra profiles compared with the findings from the analytical FR analysis given in Table 4.1. Gaussian CONS was used in the analysis because it has a flat spectra, that is, its energy is distributed uniformly across all frequency channels, which makes it easier to understand the system frequency characteristics.

As discussed earlier, determining similarity relationships between variables is key to the process of signature formulation. Clustering being a well established technique for assessing similarity relationships between objects (or time series in this case), it was therefore applied here. Details on clustering are provided in the Methodology chapter (Section 3.8) and therefore not repeated here. Suffice to say that a hierarchical clustering method was used, in which the order in which the cluster-sub cluster relationships are formed at different stages is graphically displayed as a tree like diagram called dendogram. For branches which are separated and join far apart, distinct clusters of the variables attached to the branches can be identified. Figure 4.1 depicts the time series and spectra profiles of the variables and their clustering for MTO and MTS systems with delay $\left(T_{p}\right)$ of 7 excited with Gaussian CONS. The time series profiles are all normalised (i.e. mean centred and amplitude scaled, the latter with respect to $\sigma$ ) while the spectra profiles are scaled with respect to the largest spectral peak because this enables better visualization of the frequency characteristics. Also, the spectra profiles can be seen to stop at $\mathbf{0 . 5}$ on the frequency axis. This is because the Nyquist sampling theorem requires a sinusoidal signal to be sampled at least twice per cycle (Chatfield, 1996).

\subsubsection{Simulation output analysis of MTO systems}

The profiles of variables from simulated MTO systems are shown in the first two rows of Figure 4.1. The time trends of the system variables (terms defined in this section earlier) 
Normalised time series

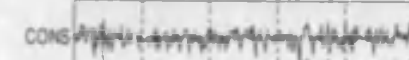

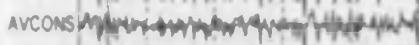

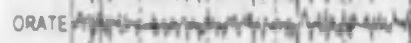

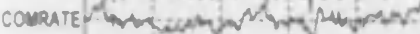

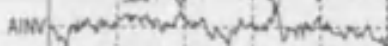

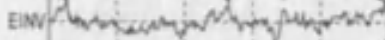

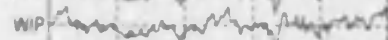

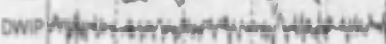

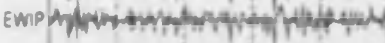

$$
0.50 \frac{100}{150} 200 \quad 250
$$

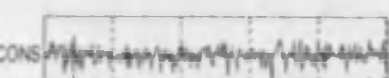

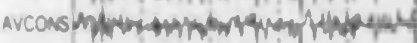

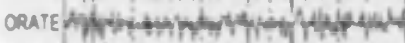

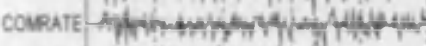

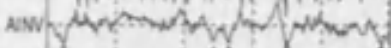
Ewh miving Mm Anim

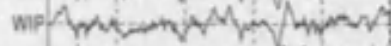

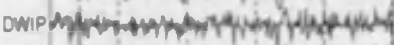

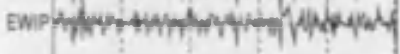

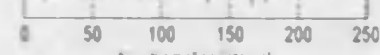

romited nend
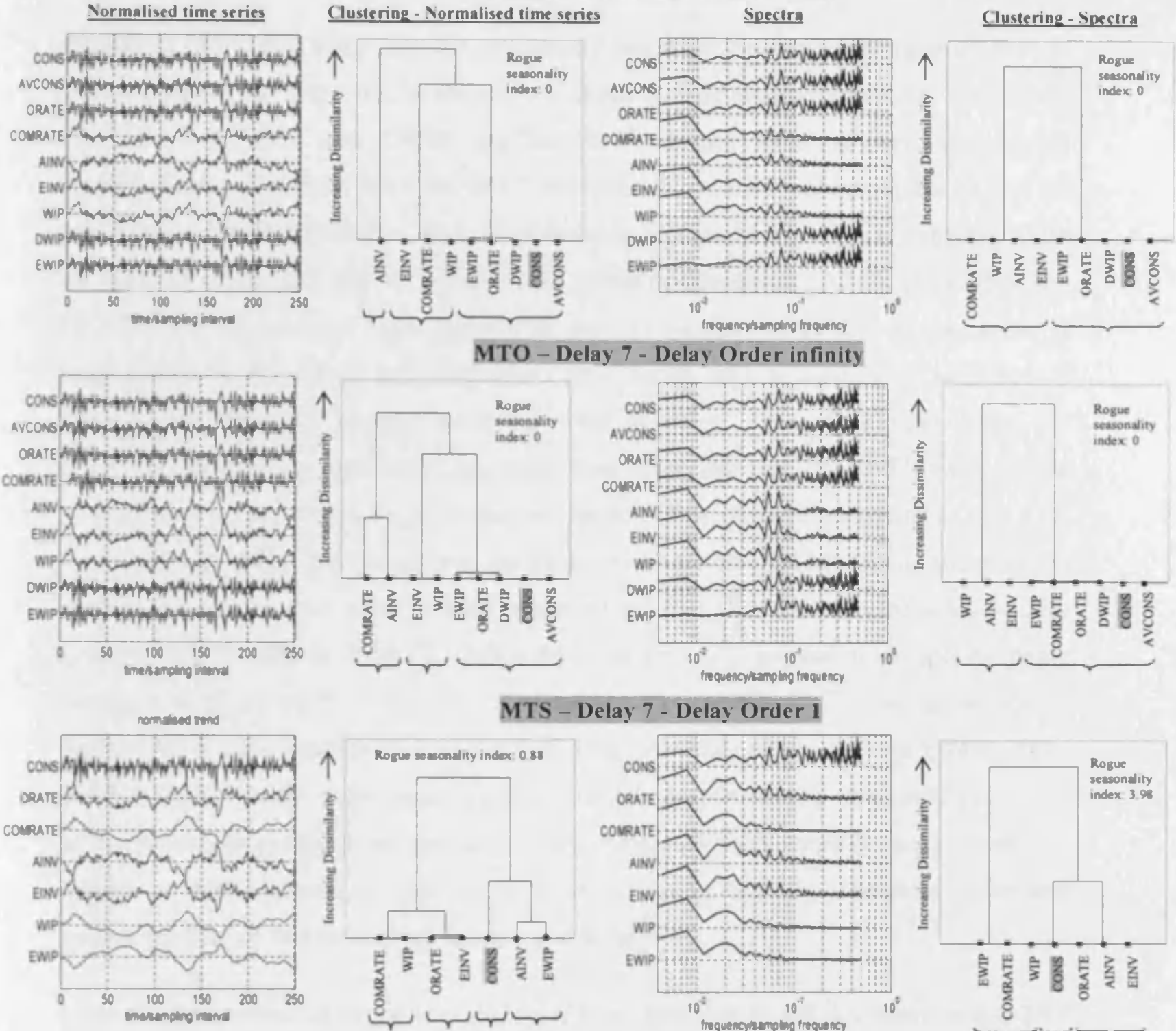

MTO - Delay 7 - Delay Order infinity
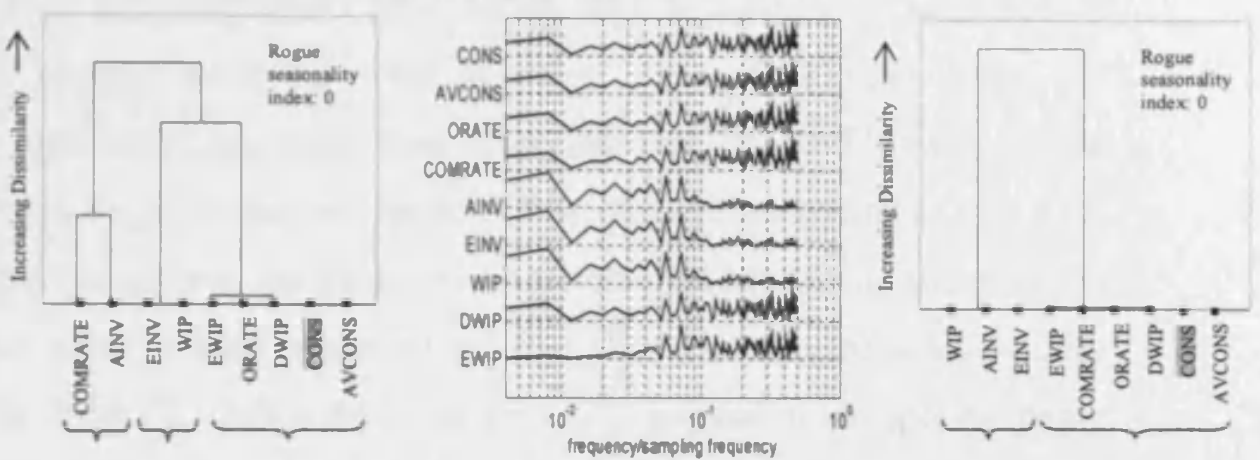

MTS - Delay 7 - Delay Order 1

\section{MTS - Delay 7 - Delay Order infinity}
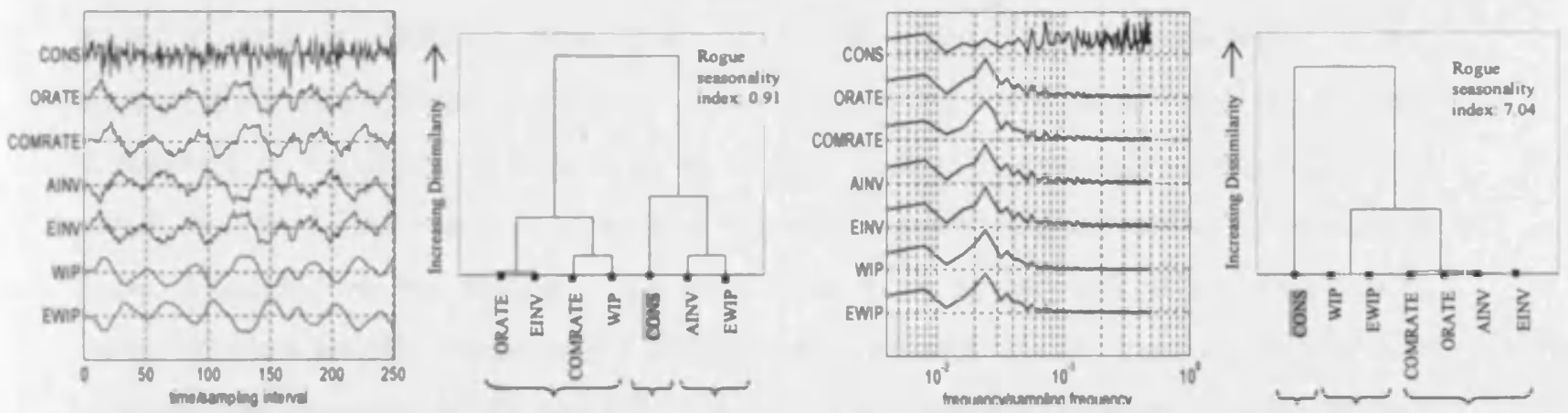

Figure 4.1 Time series, spectra and their clustering for single echelon MTO and MTS systems excited by Gaussian demand (CONS) 
are shown in the first panel and the spectra derived from Fourier transformation (FT) in the third panel. An inspection of these plots shows a close similarity between the CONS, ORATE, AVCONS and DWIP profiles, in both the time as well as spectral representations. Based on the APIOBPCS structure (Figure 3.2 and Appendix A) and the parameters of the MTO system, such a relationship between the profiles is expected. With no demand smoothing and no pipeline and inventory feedback CONS, AVCONS and ORATE are equivalent. Their profile in turn is similar to DWIP as the latter is proportional to AVCONS and normalised time series data is used. The behaviour of COMRATE, however, changes with the order of delay. For a first order delay, the production or delivery pipeline acts as a low pass filter, that is, COMRATE transmits the low frequencies and filters the high frequencies in CONS. This behaviour of COMRATE for an order of delay one system is as per its decreasing analytical FR profile and which is evident in both its time series as well as spectra profiles. For the order of delay infinity system, COMRATE is ORATE shifted by lead time $T_{p}$, so that their spectra being invariant to phase shifts in the time domain, are similar. Finally, it was mentioned in Section 4.1.1 that variables such as EWIP/CONS (for delay order one) and EINV/CONS and EWIP/CONS (for delay order one and infinity), despite having maximum FR values of $T_{p}$ (which is greater than one and hence associated with amplification) would not behave as such because of their a-priori normalization with $T_{p}$. The time series and spectra profiles of these variables validate the same.

From the perspective of rogue seasonality, it is evident that in MTO systems, the CONS profile is transmitted to other variables without distortion, that is, other variables do not have frequencies different from those in CONS. Hence, rogue seasonality is not generated in such systems, which was seen earlier in the analytical FR analysis as well. Clustering of variables, in both time as well as spectra representation, highlights this result, that is, CONS is similar to most of the other system variables and is clustered with them. Though time and frequency domains seem to be equally effective in this case, a more detailed profile comparison highlights the disadvantage of operating in the time domain. For example, AINV and EINV, despite being similar (inverted versions of each other as DINV is constant) are clustered separately in the time domain. Similarly, the 
profiles of COMRATE and ORATE in a delay order infinity system, being delayed versions of each other are actually similar but still clustered separately in the time domain. The spectra representation correctly recognizes the similar nature of variable profiles in both cases.

\subsubsection{Simulation output analysis of MTS systems}

The profiles of variables from a simulated MTS system are shown in the bottom two rows of Figure 4.1. All the variables except CONS are seen to oscillate at similar frequencies in each of MTS delay order one and delay order infinity systems. Though this is apparent in the time profiles, the spectra profiles highlight the same more clearly. The behaviour of the profiles, are also as expected from their analytical FR analysis given in Table 4.1. For example, the frequency of oscillation of variables in an order of delay one system, as evident from peaks in their spectra is 0.02 , which is equivalent to the frequency associated with maximum amplification $\left(\omega_{\max } \mathrm{FR}\right)$ suggested by the analytical FR analysis.

It is to be noted that the analytical FR analysis is in terms of angular frequency $(\omega)$ and therefore needs to be divided by $2 \pi$ to relate it to the abscissa in the spectra plot, which is in terms of frequency. The oscillation frequency of variables in the delay order infinity system at 0.03 , evident from their time series plots and peaks in their spectra is also equivalent to that expected from their analytical FR analysis. The analytical FR analysis also suggested that the peak amplification of system variables in an MTS delay order infinity system is greater than that in the delay order one system. The time series and spectra profiles of these systems validate this as well. The spectra of variables in the delay order infinity system show a higher spectral peak and less distribution of energies in other frequency channels than delay order one. Variables in the former also show more consistent cyclicality than the latter. Hence overall, the good correspondence between the simulation output and the findings of the analytical FR analysis effectively verifies the simulation model used.

Table 4.1 also clearly shows the significant smoothing of other operating variables compared to CONS in MTS systems. This is evident in both the time and frequency 
domains (spectra) and can be intuitively explained on the basis of the system structure and control policies used. ORATE in a MTS system depends upon AVCONS and EINV. AVCONS is based on a long term forecast and is not updated regularly based on CONS while EINV is reduced by a factor of $T_{i}$ (time to adjust inventory), when used to compute ORATE. Hence, ORATE is not influenced directly by CONS but only indirectly through a smoothed EINV. This is what causes dissimilarity between ORATE and CONS which gets passed on to create dissimilarity between other variables and CONS also.

The cluster profiles in Figure 4.1 clearly highlight the nature of clustering of CONS as a discriminating factor between presence and absence of rogue seasonality. Comparing rows 1 and 2 with 3 and 4 in Figure 4.1 shows that, while CONS is clustered with the other variables in the MTO systems where no rogue seasonality is present, it is clustered separately from the other variables in the MTS systems associated with rogue seasonality. Also seen in MTS systems, is other variables being clustered together which is because of similarity from generation of cyclicality (rogue seasonality) of a similar frequency. The extent of CONS dissimilarity in MTS systems also indicates the intensity of rogue seasonality. This is evident when we compare clustering profiles of MTS delay order one with delay order infinity system in rows 3 and 4 of Figure 4.1 .

The signature for rogue seasonality can therefore be simply expressed in terms of whether internal system variables cluster or do not cluster with CONS. When the latter is true, rogue seasonality is considered present, otherwise not. This signature based approach builds on the findings of Thornhill and Naim (2006). Multiple variables are used in deriving the signature recognizing a similar manifestation of rogue seasonality in the steel case study which they used. While Thornhill and Naim's rogue seasonality detection approach involved making assumptions and manual discretion, the signature based approach proposed here is quite general and can be applied in an automated fashion. A key question in this approach is the domain representation or feature to be used for signature development. Between time and spectra representations, the signature developed from the latter appears to be better as seen in Figure 4.1. Rogue seasonality intensity in the MTS order of delay infinity system is greater than in the order of delay one system as in Table 4.1 and this is accurately represented in the spectra representation. 
CONS is more distinctly clustered from other variables in the order of delay infinity system than in order of delay one system. However, the signature in the time domain does not reflect the same.

\subsubsection{Index of rogue seasonality}

Rogue seasonality detection based on the signature as discussed above, has advantages over the approach suggested by Thornhill and Naim (2006). However, it still has a few deficiencies. One deficiency is that framing of the signature involves assessment of cluster membership and cluster profiles. This introduces subjectivity into the process. There are further complications from issues such as the dissimilarity scale used in cluster visualisation. Also, the way the signature is defined, it appears to be more appropriate for a binary $\left(\begin{array}{ll}0 & 1\end{array}\right)$ categorisation of rogue seasonality i.e. whether rogue seasonality was present or absent. However, in reality, rogue seasonality is present in most supply chains (Kim and Springer, 2008), but with different intensities. A numerical indicator of rogue seasonality intensity, and which would also enable its detection would be useful and was therefore explored.

This alternative approach involves defining an index based on the similarity relationships between the supply chain variables. The index definition is based on the hypothesis that rogue seasonality generation and propagation creates significant variation in the profiles of variables. With rogue seasonality, the other variables excluding CONS such as AINV and WIP get a cyclical profile and thus become quite similar to each other, while becoming dissimilar to CONS. The dissimilarity between CONS and the other variables is therefore compared to the dissimilarity between the other variables themselves. In the case where the ratio between the two is large it indicates rogue seasonality of greater intensity for the reason given above. The formal definition of the index is as follows:

Rogue Seasonality Index $=\left(\frac{\text { Minimum dissimilarity between CONS and the other variables }}{\text { Average dissimilarity between all variables except CONS }}\right)$

Dissimilarity is measured in terms of the Euclidean distance between the variables (time or feature domain) for all the techniques except CCF, where it is measured as $(1-\max$ correlation) between two time series within specified maximum lags. The rationale for 
choosing these dissimilarity measures has been explained in the Methodology chapter. The term 'index' used in subsequent sections and chapters, refers to this definition of the rogue seasonality index.

The index as defined above is tested on MTO and MTS delay order one and infinity systems to assess its effectiveness in characterizing the intensity of rogue seasonality (see Figure 4.1). The index values in the time domain for MTO delay order one, MTO delay order infinity, MTS delay order one and MTS delay order infinity systems are $0,0,0.88$ and 0.91 respectively which accurately indicate absence, absence, medium intensity and high intensity of rogue seasonality respectively. The corresponding values using spectra (or frequency domain representation) are 0, 0, 3.98 and 7.04 which not only correctly characterize the increasing intensity of rogue seasonality but do so with greater discrimination between different intensities than in the time domain. This comparative superiority of spectra vis-à-vis time is, however, based on the analysis of a single instance, and requires validation through multiple replications.

\subsection{Rogue seasonality signature and index assessment in three echelon MTS systems with different time series transformations and features}

The rogue seasonality signature and index were understood and validated using single echelon MTO and MTS delay order one and infinity systems in the earlier section. Also, time and frequency domain representations were used to derive them, out of which the latter was found to be better. The validity of signature, index and the effectiveness of alternative time series transformations is now sought to be established with multi echelon systems. Three echelon MTS systems are therefore simulated, with these systems being created by coupling together of the corresponding single echelon systems. The coupling is done by making ORATE of the downstream echelon equivalent to the CONS of the upstream echelon. For deriving the signature and index, other time series transformations and features (besides time and spectra) discussed in Section 3.7, are also assessed. The plots based on these different feature representations for simulated order of delay one and infinity systems (MTS three echelon) are given in Figures 4.2 and 4.3 respectively. A delay of 7 at each echelon and Gaussian noise as the exogenous demand is used as in the case of the single echelon analysis. Also, the time series data is normalized before 

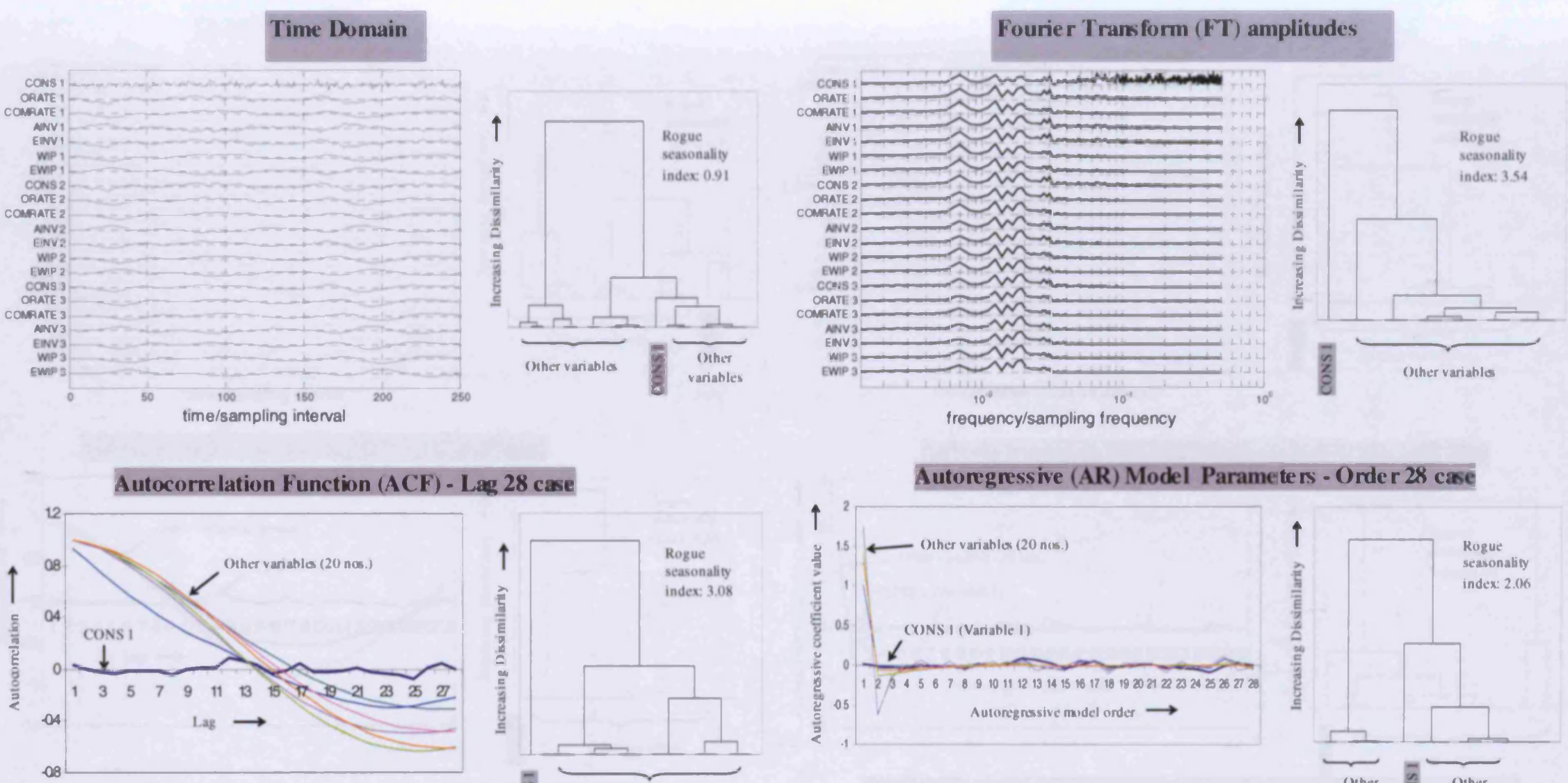

frequency/sampling frequency

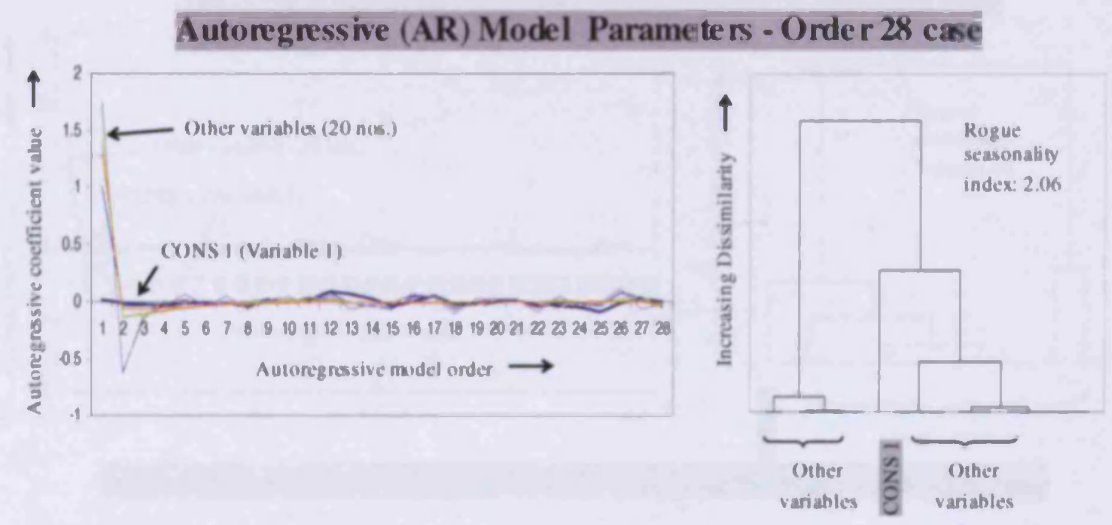

Cross correlation function (CCF) - Max \pm 28 lags case

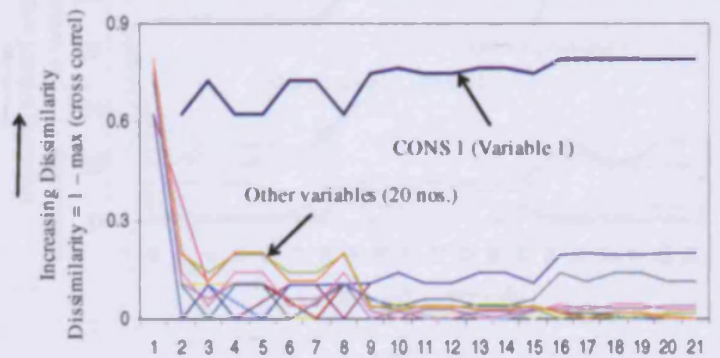

Variatle nunher $\longrightarrow$

Delay magnitude $=7$

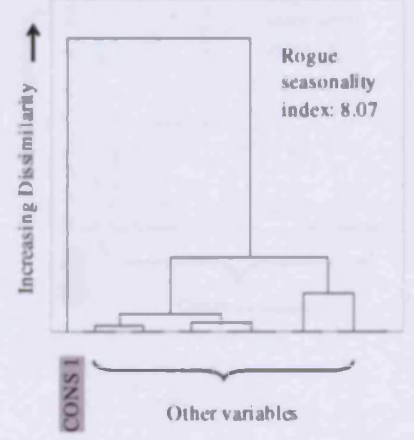

\section{Haar discrete wavelet transfer (DWT) coeficients - Level 5 case}
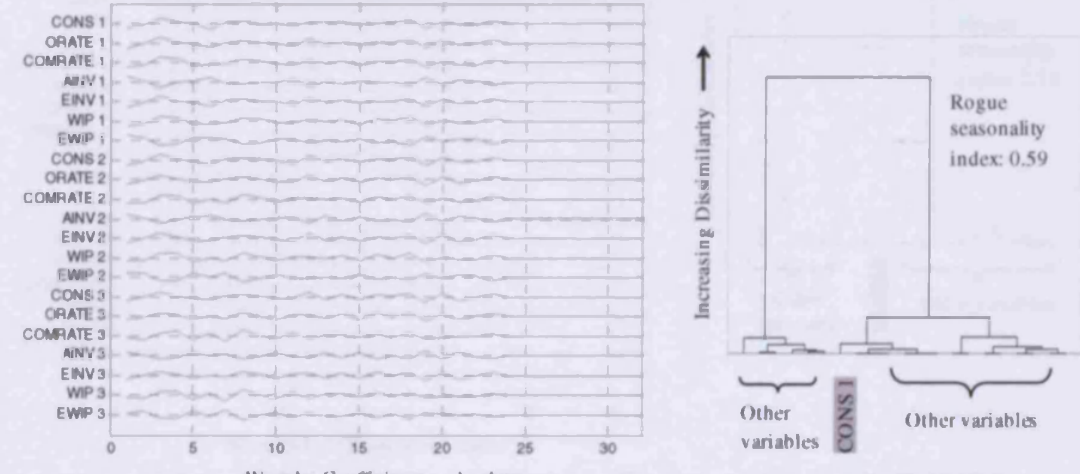

Figure 4.2. Plots and clusters using different features for a three echelon MTS system with delav order one excited by Gaussian demand (CONS1) 

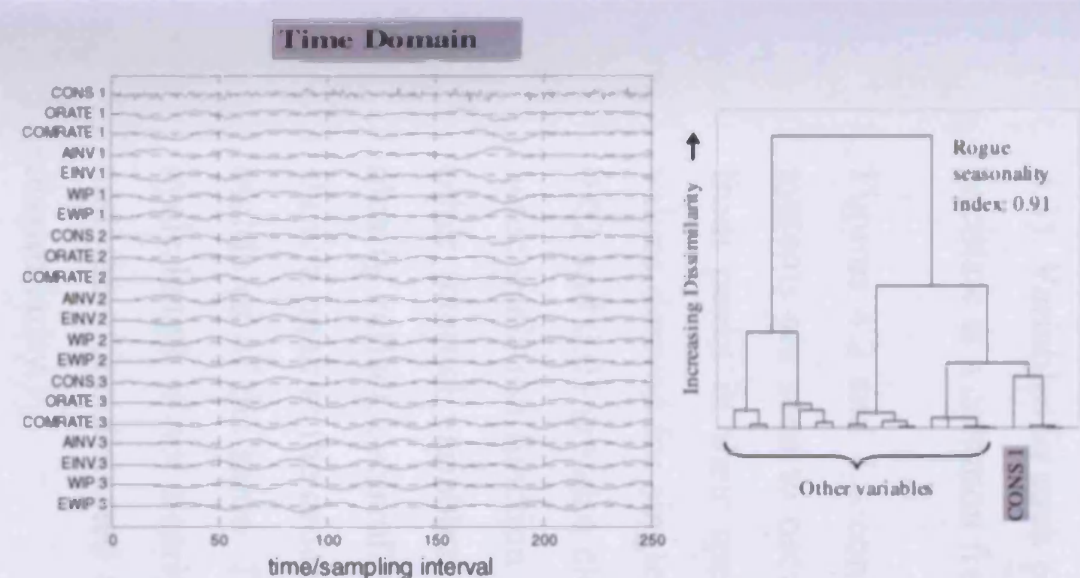

Autocorrelation Function (ACF) - Lag 28 case
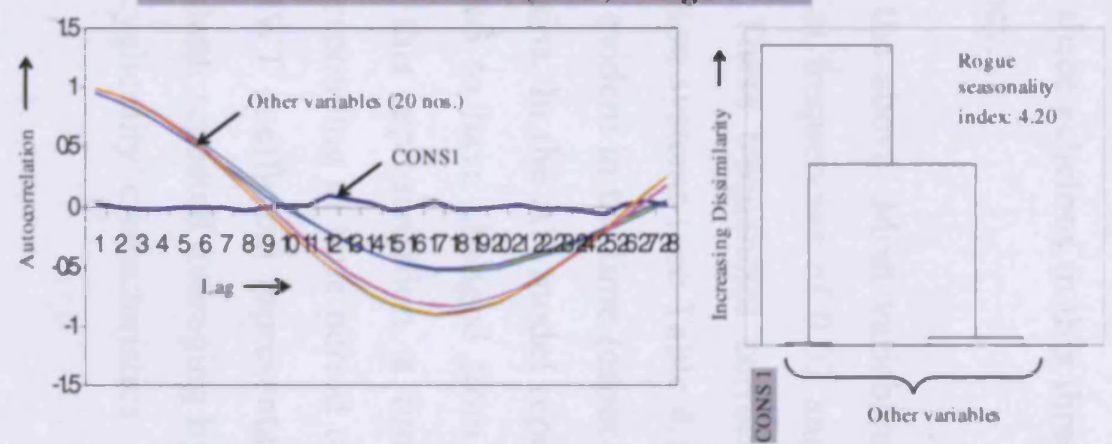

Cross corre lation function (CCF) - Max \pm 28 lags case

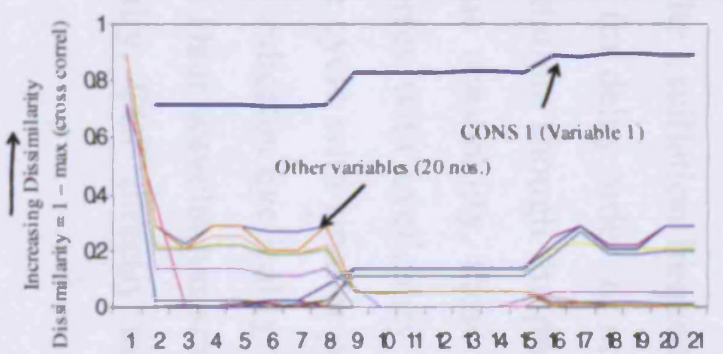

Variable number $\longrightarrow$

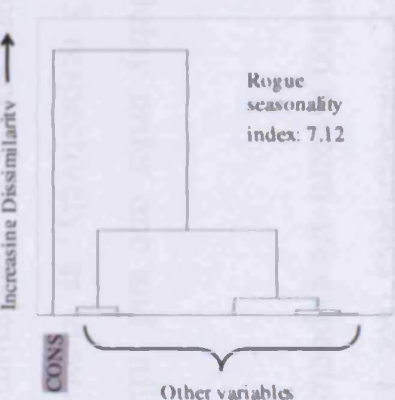

Delay magnitude $=7$
Fourier Trans form (FT) amplitudes
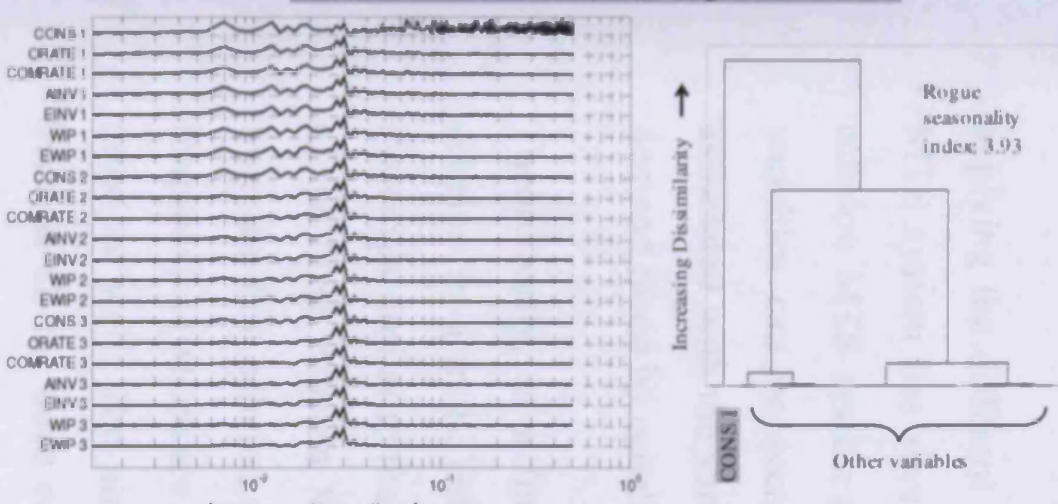

frequency/sampling frequency

Autoregressive (AR) Model Parame te rs - Order 28 case
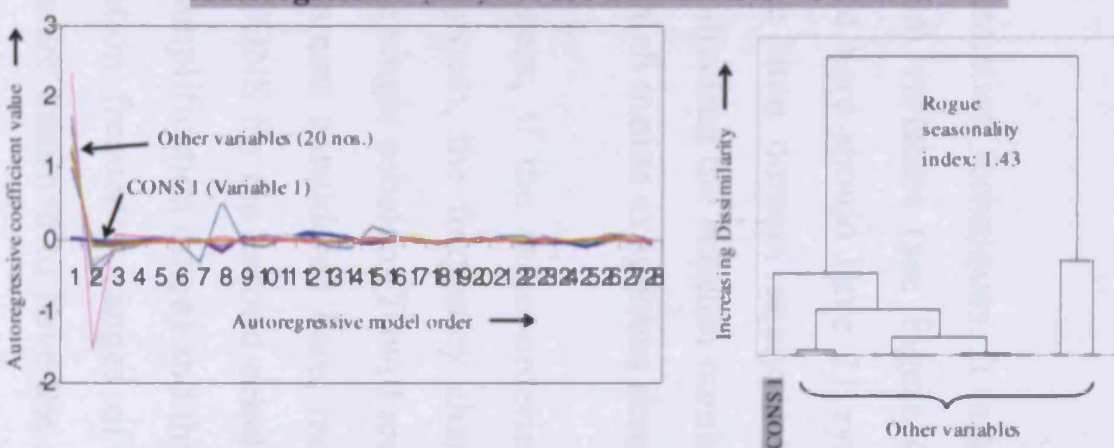

Haar discre te wavelet transfer (DWT) coefificie nts - Level 5 case
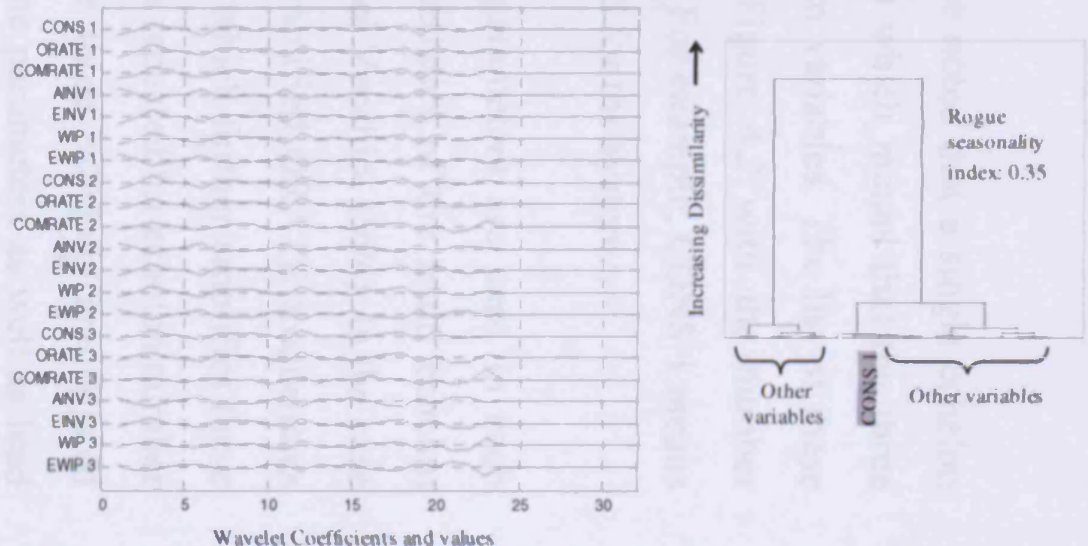

Figure 4.3. Plots and clusters using different features for a three echelon MTS system with delay order infinity excited by Gaussian demand (CONS1) 
applying the different transformation techniques. It is to be noted that a single echelon MTS system has seven system variables (see Figure 4.1) which means that the three echelon MTS system analysed here should have 21 system variables. The list of these variables can be seen in the time domain analysis in Figure 4.2 with the number associated with the variable indicating the echelon number. For example, CONS 1 means demand faced by echelon 1 which means exogenous demand for the system.

Theoretically, in a linear system, if the same ordering parameters are used in each echelon in a multi echelon system, the frequency characteristics of the multi echelon system is similar to that for the single echelon (Towill and del Vecchio, 1994). In the case of the three echelon MTS systems considered here, the amplified ORATE frequencies from the first echelon act as CONS for the second echelon which further amplifies these frequencies (which are in the amplification range) and this is repeated for echelon number three as well. The amplification frequency ranges of the three echelons are aligned because all the three echelons are assumed to have the same parameters as well as lead time $\left(T_{p}\right)$, the latter being a key determinant of the frequency characteristics (see Table 4.1). Variables in each of the three echelons in this three echelon system are expected to oscillate at a common frequency.

Figures 4.2 and 4.3 confirm the above. Most variables in delay order one and infinity systems are seen to oscillate at frequencies of 0.02 and 0.03 respectively, as identified from peaks in their spectra. These frequencies correctly correspond to maximum FR values derived for single echelon systems (see Table 4.1). The oscillation frequencies of 0.02 and 0.03 are also clearly evident in the time (especially for delay order infinity) and autocorrelation function domains. In the AR model representation, though the profile of other internal variables appears to have changed from rogue seasonality, these do not clearly reflect cyclicality. In this representation, a time series with cyclicality should show a peak at the order corresponding to time period of the cycle with smaller peaks at multiples of the same. The DWT coefficient representation indicates cyclicality but the cycle length is not clearly evident; repeated averaging by the Haar wavelet has smoothed and therefore distorted the cyclicality characteristics. Finally, CCF directly indicates dissimilarity. 
In terms of the effectiveness of each domain representation or feature in representing the rogue seasonality signature, FT amplitude, ACF and CCF are seen to be better than others for both order of delay one as well as order of delay infinity systems. In each of these cases, the exogenous demand (CONS 1 ) is distinctly clustered from the other variables, with the latter being clustered together. On the other hand, time and DWT coefficient domains, yield a signature of lesser clarity (CONS 1 is not that distinctly separated from other variables in the dendogram) with AR model features being between the two (FT, ACF, CCF and time, DWT) in terms of effectiveness of the signature derived from it.

The plots in Figure 4.2 and 4.3 also clearly depict the rationality of the rogue seasonality index for certain features. The system variables excluding CONS 1 show cyclicality, and therefore have similar profiles and low dissimilarity with each other resulting in a small denominator in the index computation. CONS 1 does not show cyclical behaviour and hence is quite dissimilar from the other variables so that the numerator in the index computation is large. Hence, when rogue seasonality is present, the index value should be a large number. This is clearly evident for FT, ACF and CCF features though the values of the indices for these as well as other features show a large variance. For example, for the MTS delay order infinity system (Figure 4.3), the index value derived in the time domain is 0.91 while it is 7.12 in the CCF domain with other features yielding index values between these two. More importantly, the index values based on some features are not consistent i.e. they do not vary as expected. For example, the MTS delay order infinity system generates rogue seasonality of greater intensity than the corresponding delay order one system (Table 4.1) and the same should be reflected in their index values derived from different features. However, only FT and ACF features i.e. index values derived from these features show this consistency; index values based on FT for delay order one and infinity systems are 3.54 and 3.93 while the corresponding values for ACF feature are 3.08 and 4.20 . Index values based on other features decrease rather than increase from the delay order one to delay order infinity system.

The above analysis demonstrated the validity of the signature and index for rogue seasonality detection along with the relative effectiveness of different features in deriving them. However, valid conclusions cannot be drawn from the analysis of just one instance 
as used here; single instance of stochastic demand from a single (Gaussian) demand process, single system structure (in terms of delay or lead time) and one set of parameters for each feature (e.g. lag 28 for ACF, order 28 for AR model). Moreover, only one definition of the rogue seasonality index was used and other definitions need to be considered to assess its stability and robustness in measuring rogue seasonality intensity. A detailed simulation experiment incorporating all these aspects was therefore conducted.

\subsection{Detailed simulation of three echelon MTS systems}

A detailed simulation analysis was done to rigorously evaluate the rogue seasonality index as a measure of rogue seasonality intensity and determine the most appropriate features and system variables for deriving it. Three sets of analyses were conducted:

1) Generate rogue seasonality of different characteristics and intensities in the system and system variables

2) Use different time series transformations and features (with different parameters for each feature) to derive the rogue seasonality index and in this way determine the best feature parameter combination for index computation (and therefore rogue seasonality detection). Time series data of system variables from step 1 are used.

3) Use different definitions of rogue seasonality index and repeat step 2.

\subsubsection{Generation of rogue seasonality of different characteristics and intensities}

Details of the simulation design used for generating time series data with different rogue seasonality characteristics and intensities are given in Table 4.2. Only the MTS system showed rogue seasonality and therefore it was the only one used in the simulation. Delay between ordering and receipt of goods, which in this case is only the production time, was varied as it plays a key role in supply chain dynamics (Forrester, 1961; Chen et al., 2000). However for MTS systems, a change in delay only changes the time period of seasonality or cyclicality and not its intensity as discussed earlier. Delay $\left(T_{p}\right)$ of 3,7 and 14 days were chosen to correspond to $1 / 2$ week, 1 week and 2 weeks delay in production. Order of delay in the system, which reflects the distribution of the output around the 
average delay, also affects the dynamics and was therefore independently varied. Orders of delay one and infinity were used, the rationale for which has been explained earlier.

Table 4.2 - Simulation design for generating rogue seasonality of different characteristics and intensities

\begin{tabular}{|l|l|}
\hline Structure and number of echelons & Linear, Three Echelons \\
\hline Supply chain control (Ordering) & Make to stock (MTS) \\
\hline Order of delay & One, infinity/pipeline \\
\hline Delay (time) & $3,7,14$ \\
\hline Demand process and parameters & $\begin{array}{l}\mathrm{AR}(1): \rho=-0.8,-0.5,0.1 \\
\mathrm{MA}(1): \theta=0.7,0.4,-0.2 \\
\mathrm{AR}(2): \rho_{1}=0.1 \rho_{2}=-0.8, \rho_{1}=0.7 \rho_{2}=-0.2 \\
\mathrm{MA}(2): \theta_{1}=0.7 \theta_{2}=-0.2, \theta_{1}=0.1 \theta_{2}=-0.8 \\
\text { Gaussian }\end{array}$ \\
\hline Exogenous seasonality & $\begin{array}{l}\text { Absent, Present (Amplitude: } 1 \text { frequency: } 0.01, \\
\text { frequency: } 0.09)\end{array}$ \\
\hline $\begin{array}{l}\text { Variables input into transformation } \\
\text { techniques/features for rogue } \\
\text { seasonality index computation }\end{array}$ & All variables, Only order and inventory variables \\
\hline l00 replications of each based on common random numbers \\
\hline
\end{tabular}

* Gaussian randomness

In terms of choice of exogenous demand, demand for many goods follow autoregressive (AR) and moving average (MA) processes of different orders (Chopra and Meindl, 2001). AR and MA of orders 1 and 2 besides random (Gaussian) demand processes were therefore considered. Within each process, parameter values such as $\rho$ 's for AR (1) and $\mathrm{AR}(2)$ and $\theta$ 's for MA (1) and $\mathrm{MA}(2)$ were varied to generate rogue seasonality of different intensities in the system variables. The underlying basis for this is as follows. The frequency response (FR) of the variables which is the ratio of the sinusoidal output to the sinusoidal input at various frequencies (Table 4.1 ) is computed relative to the exogenous demand (CONS). Therefore, if CONS had a significant low frequency component, which corresponded to the amplification frequency range, then the rogue seasonality intensity generated in the variables would be high. In contrast, if the low frequency component in CONS was low, the rogue seasonality intensity generated in the variables would also be low. The parameters within each demand process were therefore chosen in such a way as to have different proportions of the low frequency amplification 
range component so that rogue seasonality with different intensities could be generated. For this, spectra as a function of parameter values was derived for each demand process and this is explained and depicted in Appendix C. Principles given in Gottman (1981) were used in the derivation. Spectra plots for the parameters considered for each demand process highlight their different low frequency amplification range content. For example, for the AR (1) process, spectra energy in the low frequency amplification range increases with an increase in the parameter value $\rho$ from -0.8 through -0.5 to 0.1 .

Exogenous seasonality of different frequencies was added to the demand pattern to make it more realistic. Two frequencies were considered: one within the amplification range (i.e. less than $\omega_{\text {crossover) }}$ and the other beyond the amplification range (i.e. greater than $\omega_{\text {crossover }}$ ). $\omega_{\text {crossover }}$ being a function of delay (Table 4.1 ), the specific frequencies added were such that they met the within and beyond amplification range requirement for all the delays considered.

Finally, in terms of the system variables used for index computation, besides using all the variables, use of select variables (specifically order and inventory) was also used. The latter was considered because from a practical perspective many organisations may choose to share information on only a few rather than all the variables. Order and inventory are the most common variables on which information is shared amongst companies (Lee and Whang, 2000). Moreover, use of these variables has also been suggested in the context of complete assessment of the Bullwhip effect dynamics (Disney and Towill, 2003). Therefore, index was also computed using the order and inventory information of all echelons.

One hundred independent replications were generated for each case using common random numbers. The data generation was done using $\operatorname{Excel}^{\circ}$ and Matlab ${ }^{\circ}$.

4.3.2 Different features assessed for effectiveness in rogue seasonality index computation

Alternative time series transformation techniques and features were evaluated to determine the most appropriate one for rogue seasonality index computation. All the 
commonly used techniques and features as discussed in the Methodology chapter as well as in Section 4.2 were applied and evaluated. Table 4.3 describes the transformations used and the features extracted, with different parameters for each feature. Matlab $\subset$ was used for all transformations, after normalizing the data to eliminate mean and amplitude scale differences. Appendix D gives the Matlab code used for performing the time series transformations, extracting features with different parameters and using them to compute the rogue seasonality indices.

Table 4.3 - Time series transformation techniques, features and parameters for each feature used for rogue seasonality index computation

\begin{tabular}{|l|l|}
\hline $\begin{array}{l}\text { Fourier transform } \\
\text { (FT) }\end{array}$ & $\begin{array}{l}\text { Amplitudes used as features. Number of frequencies (parameters) } \\
\text { considered: } \\
\text { a) Total (FT Total) b) First 7 (FT 7) c) First 28 (FT 28) } \\
\text { d) Number with 80\% of total energy in the data series (FT 80\% Energy) }\end{array}$ \\
\hline $\begin{array}{l}\text { Autocorrelation function } \\
\text { (ACF) }\end{array}$ & $\begin{array}{l}\text { Autocorrelation upto specified lags used as features. Number of lags } \\
\text { (parameters) considered: } \\
\text { a) } 7 \text { (ACF 7) b) 28 (ACF 28) }\end{array}$ \\
\hline $\begin{array}{l}\text { Cross correlation } \\
\text { function (CCF) }\end{array}$ & $\begin{array}{l}\text { Maximum cross correlation between pairs of variables within maximum } \\
\text { lags specified, used as the feature/similarity measure. } \\
\text { Maximum lags (parameters) considered: a) 7 (CCF 7) b) 28 (CCF 28) }\end{array}$ \\
\hline $\begin{array}{l}\text { Autoregressive model } \\
\text { (AR Model) }\end{array}$ & $\begin{array}{l}\text { Coefficients of the fitted AR model, up to specified orders used as } \\
\text { features. Order of AR model (parameters) considered: } \\
\text { a) 7 (AR 7) b) 28 (AR 28) } \\
\text { c) Minimum AIC (Akaike, 1981) (AR AIC) }\end{array}$ \\
\hline $\begin{array}{l}\text { Discrete wavelet } \\
\text { transform (DWT) -Haar } \\
\text { wavelet }\end{array}$ & $\begin{array}{l}\text { Wavelet coefficients from DWT at different levels used as features. Levels } \\
\text { (parameters) considered: } \\
\text { a) } 5 \text { (DWT 5) b) Based on minimum Shannon entropy (Shannon } \\
\text { and Weaver, 1964) (DWT Shannon) }\end{array}$ \\
\hline Time series data without any transformation was used as the default for comparison (Raw Time)
\end{tabular}

Bracketed text are referred to as feature parameter combination in the rest of the thesis

a) Fourier transform (FT): Different number of frequencies (referred to as parameters) are considered to assess the sensitivity of the index/indices to the same. The FT Total option used includes amplitudes of all frequencies in the computation and in this way ensures that rogue seasonality frequencies do not get excluded. Though this option does not improve the computational efficiency vis-à-vis the time domain, it does increase the clustering accuracy from better alignment of similar but leading/lagging profiles. The 
latter point has been highlighted in the study by Thornhill and Naim (2006). Other options considered use lesser number of frequencies so as to increase the computational efficiency (from dimensionality reduction) as well as the clustering accuracy (from noise elimination; the higher frequencies not used are generally associated with noise). FT 7 and FT 28 frequency options involving the use of first 7 and 28 frequencies were considered, with 7 and 28 chosen so as to correspond to a week and month respectively. Despite their advantages, these options carry greater risk of rogue seasonality frequencies getting excluded from the analysis due to inappropriate choice of parameters (number of frequencies).

The final option, FT $80 \%$ Energy, eliminates the need to choose number of frequencies. This is chosen on the basis of the distribution of energy (square of amplitude) across different frequencies for that variable. $80 \%$ Energy refers to the fact that as many initial frequencies are used, which have cumulatively eighty percent of the total signal energy. The FT $80 \%$ Energy option eliminates the risks as in FT 7 and FT 28, of relevant frequencies associated with rogue seasonality not being considered while at the same time providing better dimensionality reduction than FT Total.

b) Autocorrelation function (ACF): ACF's were calculated for 7 and 28 lags (referred to as ACF 7 and ACF 28 respectively) to assess sensitivity of the results to the number of parameters while ensuring correspondence with other features which also use 7 and 28 terms in the index computation.

c) Cross correlation function (CCF): In this method the similarity between two time series was defined to be the absolute maximum correlation between them within the maximum lags specified. The maximum lags specified were 7 and 28 (referred to as CCF 7 and CCF 28) similar to other methods which meant that the cross correlation function was evaluated within \pm 7 and \pm 28 lags.

d) Autoregressive model (AR model): In this method an AR model is generated for each time series up to a specified order with the coefficients of the model then used for index computation. Orders of 7 and 28 (referred to as AR 7 and AR 28) were considered for the same reasons as other techniques. An approach based on the Akaike Information 
Criteria (AR AIC) (Akaike, 1981) which does not require a-priori specification of model order was also considered. Here optimal order is automatically chosen by trading off model order with error in model fitting based on the equation given below. However, in an analysis of multi variables, the optimal order for individual variables could be different. For such cases the maximum optimal order across variables was used as the order for each variable.

Optimal order $=\min (A I C)$ where $A I C=n\left[\ln \left(\sum_{i=1}^{i=n} \varepsilon_{i}^{2} / n\right)\right]+2 k$

$k=$ number of parameters in the statistical model (model order in this case)

$n=$ number of observations and $\varepsilon_{i}$ is the error between observation and fitted model

e) Discrete Wavelet Transform (DWT)-Haar: Wavelet coefficients, which essentially represent the correlation between the original and the local oscillation represented by the wavelet basis function (Percival and Walden, 2000) were used for index computation in this method. Signals could be decomposed to different levels before computation of the index, details of which are explained in the Methodology chapter. Level 5 (referred to as DWT 5) was chosen, as it gives 32 approximation coefficients, which is close to the 28 coefficients used in other methods. A higher level, to correspond to 7 coefficients in other methods, was not used as the approximation coefficients were excessively smoothed. A parameter free approach was also tried in which the appropriate level could be automatically selected on the basis of data characteristics i.e. did not require a priori level specification. This was based on the Shannon entropy function (Shannon and Weaver, 1964) which can be regarded as a quantitative measure of uncertainty. At each level, signal energies are used to evaluate this entropy function with the level selected being the one where its value is the minimum. This level corresponds to maximum information availability as information is equivalent to the removal of uncertainty.

Level of entropy $(H)=-\sum_{k} E_{j k} * \log E_{j k} ; E_{j k}$ is the wavelet energy at scale $j$ and instant $k$

This can also be represented as $H=-\sum_{i=1}^{N} s_{i}^{2}{ }^{*} \log s_{i}^{2} ; s:$ signal and $s_{i:}$ wavelet coefficients 


\subsubsection{Different rogue seasonality index definitions considered}

The rogue seasonality index has been defined earlier in Section 4.1.3 on page 98. The effectiveness of this definition of the rogue seasonality index has been demonstrated earlier. However, in certain cases it may not indicate rogue seasonality accurately. For example, there could be a case where rogue seasonality is present in the supply chain and such cyclicality is evident in most variables; however, there are few variables which are not showing cyclicality, as a result of which they remain similar to CONS, causing the index value to be low due to a small numerator and thus giving an erroneous indication of rogue seasonality intensity. To eliminate this possibility, an alternative definition of the rogue seasonality index called Alternate 1 or Alt 1 is proposed, which considers average rather than minimum dissimilarity between CONS and the other variables in the numerator. Finally, a third definition of the rogue seasonality index called Alternate 2 or Alt 2 is proposed, which incorporates the inherent variability in the variables by using standard deviation.

(Alternate 1 or Alt I) $=\left(\frac{\text { Average dissimilarity between CONS and the other variables }}{\text { Average dissimilarity between all variables except CONS }}\right)$

(Alternate 2 or Alt 2) $=\left(\begin{array}{l}\text { Minimum dissimilarity between CONS and the other variables } \\ \text {-Average dissimilarity between all variables except CONS } \\ \text { Standard deviation of dissimilarity between all variables except CONS }\end{array}\right)$

In the simulation analysis, computations are made considering each of the three definitions. The Matlab® code used for computing these indices is given in Appendix D.

\subsection{Analysis of output from detailed simulation of three echelon MTS system}

The output of the detailed simulation is essentially the index values based on different feature parameter combinations for each simulated system. Taking the example of an MTS system with average delay 3 and order of delay 1 , excited with one instance of AR (1) demand process with $\rho 0.1$, the rogue seasonality index is calculated based on each of raw time, FT Total, FT 7, FT 28, FT 80 \% Energy, AR 7, AR 28, AR AIC, ACF 7, ACF 28, CCF 7, CCF 28, DWT (Level 5) and DWT (Level Shannon) feature parameter 
combinations. This is replicated 100 times to calculate the average as well as coefficient of variation (standard deviation/average) values for each combination. Although this exercise is done for each of the three index definitions (index, Alt 1 and Alt 2), output for only the index (equation 4.1) is presented in order to reduce the complexity from excess data. However, the outputs from the three indices are compared, with the extent of correlation between them indicating the robustness of the logic of the index.

The simulation output is discussed in the following sequence. First the case where exogenous demand does not have seasonality and all the variables are used in index computation is analysed. Next the exogenous demand with seasonality case is analysed; the impact of seasonality on the index is discussed together with the changes required to ensure the index's continued validity as a measure of rogue seasonality intensity. Finally, the case where only order and inventory variables are used in the index computation is discussed.

\subsubsection{Case I: Exogenous demand without seasonality and all variables used}

\subsubsection{General observations}

Table 4.4 gives the index values for the AR (1) demand process for this case with those for other demand processes given in Appendix $E$, these values having been computed using different feature parameter combinations. Though AR (1) is used as the basis for analysis, any of the other demand processes could also have been used instead.

The index values for all feature parameter combinations are seen to be greater than zero. A zero value is associated with a system such as MTO that does not exhibit any rogue seasonality. Since only MTS systems are simulated, and all such systems with non zero lead time exhibit rogue seasonality, this signifies that all feature parameter combinations could be used to compute the index. However, the index values across different combinations show a large variance, with the values for some combinations being particularly low. 
Table 4.4 - Rogue seasonality index for a three echelon MTS system based on different feature parameter combinations; All variables used in computation of the index

\section{Demand Process: AR (1)}

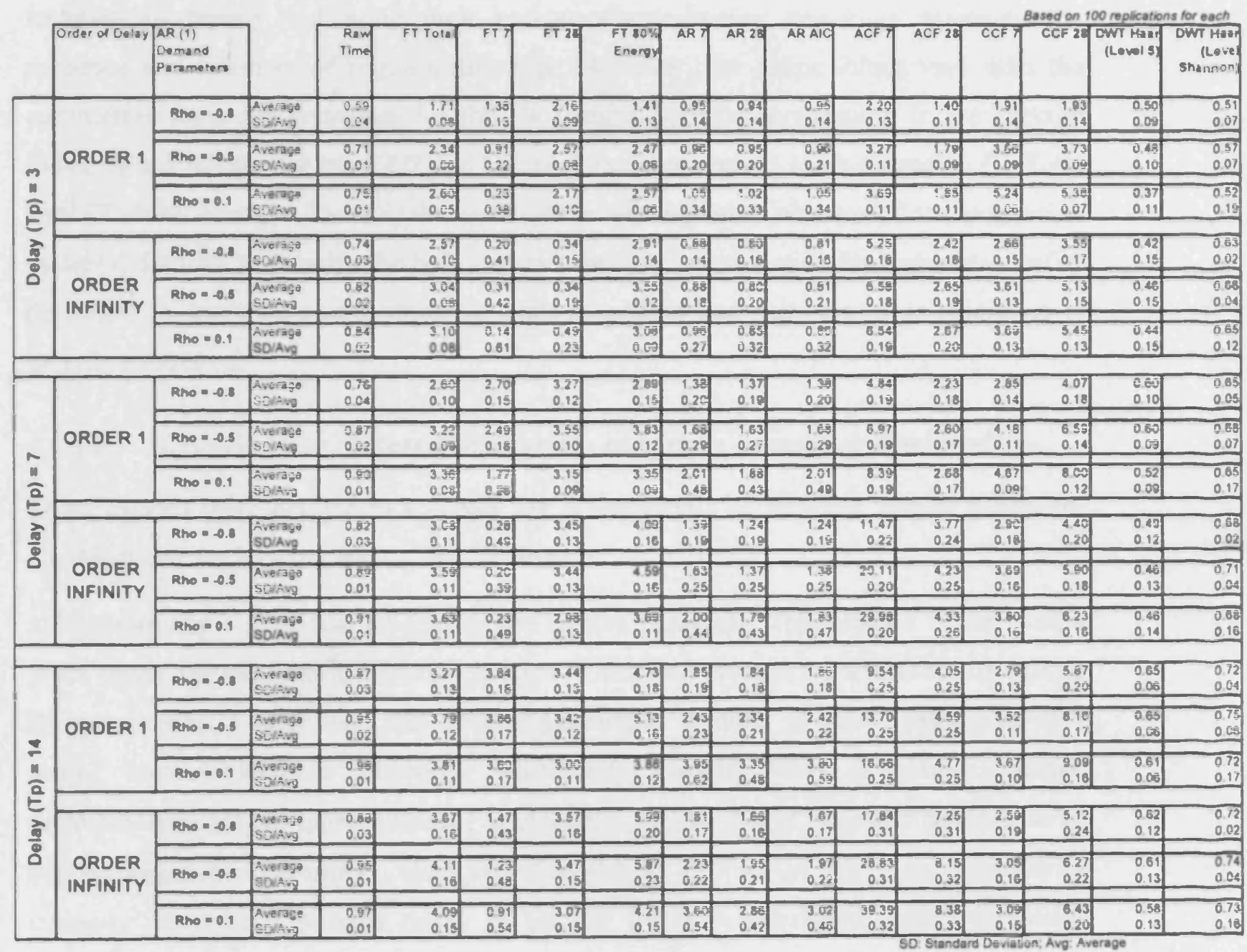

The index value from the time domain is less than 1 for all demand-delay combinations. The magnitude of change in the index value, from changes in demand parameter/order of delay in this domain is also small. A specific case can be seen in Figure 4.1 where MTS order of delay one and order of delay infinity cases have index values based on the time domain of 0.88 and 0.91 . This is not surprising as data in the time domain is unable to identify similarities in leading/lagged profiles, which makes the average dissimilarity among the other variables (excluding CONS) much larger than that between CONS and these variables. A similar low index value is observed from use of DWT coefficients. 
The index values obtained from the AR model features have reasonable magnitudes, though they have a higher proportion of values less than one as compared to other features. The largest index values are obtained using features from the FT, ACF and CCF techniques, thereby indicating their greater discriminating power in assessing the presence and intensity of rogue seasonality. However, the index values vary with the parameters used. For example, for the FT feature, significant volatility in the average index values is seen for the FT 7 and FT 28 parameter options as compared to FT Total and FT $80 \%$ Energy. Overall, the wide range of responses obtained from simulation makes it difficult to identify the best feature parameter combination for rogue seasonality detection. A systematic and objective basis to collate and compare these responses is therefore required.

\subsubsection{Criteria for structured comparison of feature parameter combinations}

Three criteria were defined to compare the effectiveness of different feature parameter combinations for index computation. These are:

a) Consistency - An ideal or appropriate feature parameter combination is that which gives index values, consistent with changes in demand parameters and order of delays. By consistency, it is implied that demand parameters or order of delays associated with actual increase/decrease in rogue seasonality, would show a similar change (increase/decrease) in index values. For example, for the AR (1) demand process, as $\rho$, the autoregressive parameter, changes from -0.8 to -0.5 to 0.1 the rogue seasonality intensity increases (discussed earlier in Section 4.3.1). A consistent feature parameter combination from a demand perspective would therefore be that, which yields index values which change similarly with $\rho$. A similar behaviour is expected for other demand processes such as AR (2), MA (1) and MA (2), in each of which the parameters chosen are such as to generate rogue seasonality of different intensities. In terms of consistency based on order of delay, a system with an order of delay infinity produces rogue seasonality of greater intensity than order of delay one, as discussed earlier. A consistent feature parameter combination from an order of delay perspective would yield index values that vary similarly with order of delay; lower index value for delay order one and higher index value for delay order infinity. 
b) Magnitude of index (Discrimination) - An ideal or appropriate feature parameter combination is that which yields index values of greater magnitude than other feature parameter combinations for the same system. This increases the discrimination between presence/absence of rogue seasonality as well as between rogue seasonalities of different intensities. A ranking system was used, where for each demand parameter, delay and order of delay combination, the feature parameter combinations were ranked based on their average index values. The sum of ranks for all parameter, delay and order of delay combinations was used as the basis for classification; the lower the sum of ranks the better was that feature parameter combination on the discrimination criteria.

c) Coefficient of variation of index (Stochastic robustness) - An ideal feature parameter combination is that which yields index values with smaller coefficient of variation, and hence, is more robust to stochastic variations than other combinations. A ranking system similar to magnitude of index was used, with the only difference being that a higher rank was given for the feature parameter combination having a lower coefficient of variation value.

\subsubsection{Comparison of feature parameter combinations based on defined criteria}

Using the index values (average and coefficient of variation) given in Table 4.4 and Appendix $\mathrm{E}$, the different feature parameter combinations were analysed in a structured way based on the above criteria. A summary of this analysis is given in Tables $4.5 a, b, c$.

Table 4.5a gives the consistency of the index with respect to demand parameters and order of delays for different feature parameter combinations. The shaded cells indicate the maximum (best) values for that row. The maximum possible consistency values used in the tables are derived as follows. For example, for AR (1), its maximum consistency for demand parameters is 12 , because use of three demand parameters $(\rho=-0.8,-0.5$ and $0.1)$ means two comparisons for each of three delays $(3,7,14)$ and two order of delays (order 1 and infinity). Similarly, its maximum consistency for order of delays is 9 as there are two orders of delays implying one comparison for each of the three demand parameters and three delays. These were similarly arrived at for other demand processes. The table shows that the FT and ACF based features yield the most consistent index 
Table 4.5: Structured evaluation of feature parameter combinations used for rogue seasonality index computation in a three echelon MTS system; All variables used

\section{Table 4.5a}

Highlighted indicates best

Consistency of rogue seasonality index for different demand processes and order of delays (Number of cases)

\begin{tabular}{|c|c|c|c|c|c|c|c|c|c|c|c|c|c|c|c|}
\hline $\begin{array}{l}\text { Nature of } \\
\text { Demand } \\
\text { Proceses }\end{array}$ & Consistency Basls & $\begin{array}{l}\text { Raw } \\
\text { Time }\end{array}$ & FT Total & FTT & FT 28 & $\begin{array}{l}\text { FT 80\% } \\
\text { Energy }\end{array}$ & ART & AR 28 & AR AIC & ACF T & ACF 28 & CCF 7 & CCF 2E & $\begin{array}{l}\text { DWT Haar } \\
\text { (Level s) }\end{array}$ & $\begin{array}{l}\text { OWT Haal } \\
\text { (Level } \\
\text { Shannon) }\end{array}$ \\
\hline \multirow{2}{*}{ AR 1} & Oemiand paramelers & $12 / 12$ & $11 / 12$ & 3/12 & $3 / 12$ & 6/12 & $11 / 12$ & $11 / 12$ & 11/12 & $11 / 12$ & $12 \pi 12$ & $12 \pi 2$ & $12 \pi 2$ & $1 / 12$ & $6 / 12$ \\
\hline & Order of delay & $7 \pi$ & $9 / 9$ & $\infty$ & $4 / 9$ & $\therefore$ & 10 & of & O/9 & $9 / 9$ & eq & $2 \pi$ & 40 & $1 / 2$ & $7 / 9$ \\
\hline \multirow{2}{*}{ MA 1} & Demend perameters & 12212 & स्या & Q12 & $6 \longdiv { 1 2 }$ & $7 / 12$ & Q12 & Q12 & $8 / 12$ & $11 / 12$ & $12 / 12$ & $11 / 12$ & $11 / 12$ & Q12 & $10 / 12$ \\
\hline & Croer of deley & 79 & 991 & 00 & 49 & 팍 & 10 & $\infty$ & o/9 & $9 / 9$ & $\infty$ & 39 & 509 & $5 \%$ & 70 \\
\hline \multirow{2}{*}{ AR 2} & Demand parametars & $5 / 6$ & $6 / 1$ & 3ब & $1 / 8$ & $5 / 8$ & $3 / 6$ & $2 \mathrm{a}$ & $2 \sqrt{6}$ & $6 / 6$ & हल & $5 / 6$ & Eict & 1ब网 & 800 \\
\hline & Orowe of delay & $4 / 6$ & $6 / 6$ & $1 / \mathrm{B}$ & 3/6 & Eज & $2 / 6$ & $1 / 3$ & $1 / 6$ & $6 / 6$ & $6 / 6$ & $2 / 6$ & $3 / 6$ & $3 / \sqrt{2}$ & $6 / 8$ \\
\hline \multirow{2}{*}{ MA 2} & Demend paramaters & 8\% & a) & $2 \pi$ & 0,5 & $3 / 6$ & $1 / 6$ & $1 / 3$ & $1 / 6$ & $6 / 6$ & 66 & कृ & $\therefore$ & $0 / 3$ & 0,6 \\
\hline & Order of delay & $4 / 6$ & a] & ar & 3,8 & $8 / 3$ & $0 / 6$ & 0, & 0.6 & $6 / 8$ & 6/ & $1 / 6$ & $2 / 0$ & $1 / 6$ & $5 / 6$ \\
\hline \multirow[t]{2}{*}{ Gaussian } & Order of delay & $2 / 3$ & 39 & $1 / 3$ & $1 / 3$ & $3 / 3$ & $0 / 3$ & $0 / 3$ & 0,3 & $3 / 3$ & $\sqrt{3}$ & $0 / 3$ & $1 / 3$ & $1 / 3$ & $2 / 3$ \\
\hline & $\begin{array}{l}\text { Overall Consiatency - } \\
\text { Demand and Order of } \\
\text { delay }\end{array}$ & (2)/6) & Bent & 1460 & $28 \pi 09$ & 34/69 & 25/69 & $21 / 89$ & $21 / 69$ & 67/60 & eartul & 42109 & 60/69 & 17169 & 4 \\
\hline
\end{tabular}

\section{Table 4.5b}

Rank analysis based on the magnitude of rogue seasonality index

(For assessing the discrimination ability of the index derived from different feature parameter combinations)

\begin{tabular}{|c|c|c|c|c|c|c|c|c|c|c|c|c|c|c|}
\hline & $\begin{array}{l}\text { Rave } \\
\text { Time }\end{array}$ & FT Total & FT 7 & FT 28 & $\begin{array}{l}\text { FT 80\% } \\
\text { Energy }\end{array}$ & ART & $\frac{\text { Larger t }}{A R 2 E}$ & $\frac{\text { he rogue }}{\text { AR AIC }}$ & $\frac{{ }^{\text {Seasona }}}{\mathrm{ACF}}$ & lity index & $\begin{array}{l}\text { igher th } \\
\text { CCF } 7\end{array}$ & $\frac{e \text { rank is }}{\text { CCF } 2 E}$ & $\begin{array}{l}\text { Used as basi } \\
\text { DWr Haen } \\
\text { (Level o) }\end{array}$ & $\begin{array}{l}\text { DWT Haar } \\
\text { (Leve } \\
\text { Shannon) }\end{array}$ \\
\hline $\begin{array}{l}\text { Sum of Ranks - AR (1) } \\
\text { Minimum / best possible } 18\end{array}$ & 203 & 86 & 185 & 128. & 74 & 144 & 183 & 159 & 22. & 89 & 89 & 39 & 243 & 224 \\
\hline $\begin{array}{l}\text { Sum of Ranks - MA (1) } \\
\text { Minimum I bex possible } 18\end{array}$ & 204 & 102 & 191 & 125 & 80 & 133 & 173 & 151 & 24 & 104 & 90 & 34 & 242 & 224 \\
\hline $\begin{array}{l}\text { Sum of Ranks - AR (2) } \\
\text { Minimum / bos possiole } 12\end{array}$ & 133 & 50 & 120 & $B 0$ & 71 & 97) & 121 & 112 & 18 & 58 & 57 & 23 & 163 & 150 \\
\hline $\begin{array}{l}\text { Sum of Ranks - MA (2) } \\
\text { Minimum / Do: posendele } 12\end{array}$ & 136 & 64 & 130 & 84 & 40 & 98 & 118 & 113 & 17 & 50 & 57 & 25 & 161 & 149 \\
\hline $\begin{array}{l}\text { Sum of Ranks - Gaussian } \\
\text { Minimum / beak posciole } 6\end{array}$ & 65 & 31 & 71 & sol & 25 & 45 & 61 & 54 & ㅇ. & 28 & 25 & 12 & 81 & 74] \\
\hline $\begin{array}{l}\text { Sum of Ranks - TOTAL } \\
\text { Minimum I best posstolo } 60\end{array}$ & 741 & 364 & 707 & 487 & 308 & 618 & 560 & 689 & 23 & 338 & 318 & 133 & 890 & 821 \\
\hline
\end{tabular}

For every demand process, delay and order of delay combination, each feature parameter combination is ranked relative to others, with the highest rank allotted to that with the largest

magnitude of rogue seasonality index (as these would provide better discrimination in rogue seasonality assessment). These ranks are then summed up first by each demand process

woch as AR (1). MA (1) and then sall together to denve a total sum of renks. Feeture parameter combinations with a lower totai sum of ranks are considered better

\section{Table 4.5c}

Rank analysis based on the coefficient of variation of rogue seasonality index

(For assessing the stochastic stability of the index derived from different feature parameter combinations)

\begin{tabular}{|c|c|c|c|c|c|c|c|c|c|c|c|c|c|c|}
\hline & $\begin{array}{l}\text { Ravy } \\
\text { Time }\end{array}$ & FT Total & FTT & FT 28 & $\begin{array}{l}\text { FT 80\% } \\
\text { Energy }\end{array}$ & AR 7 & AR 29 & AR AIC & ACF $T$ & ACF 20 & CCF & CCF 2E: & $\begin{array}{l}\text { DWT Haar } \\
\text { (Level of) }\end{array}$ & $\begin{array}{l}\text { DWT Haer } \\
\text { (Leve) } \\
\text { Shannon) }\end{array}$ \\
\hline $\begin{array}{l}\text { Sum of Ranks - AR (1) } \\
\text { Minimum / bes posesible } 18\end{array}$ & 21 & 63 & 215 & $\infty$ & 100 & 202 & 187 & 211 & 106 & 191 & 108 & 153 & 83 & 68 \\
\hline $\begin{array}{l}\text { Bum of Ranks - MA (1) } \\
\text { Minimum I berk possible } 18\end{array}$ & 18 & 64 & 213 & 103 & 98 & 210 & 192 & 217 & 181 & 100 & $\infty$ & 134 & $\infty$ & 81 \\
\hline $\begin{array}{l}\text { Sum of Ranks - AR (2) } \\
\text { Minimum I bex poseible } 12\end{array}$ & 12. & 43 & 120 & 67 & 73 & 145 & 141 & 155. & 116 & 120 & 57 & 88 & 61 & 53 \\
\hline $\begin{array}{l}\text { Sum of Ranks - MA (2) } \\
\text { Minimum I bext posesible } 12\end{array}$ & 12 & 43 & 180 & 70 & 68 & 132 & 128 & 134 & 125 & 138 & 65 & 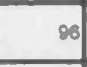 & 54 & 47 \\
\hline $\begin{array}{l}\text { Sum of Ranks - Baussian } \\
\text { Minimum / box posebiel } 6 \text {. }\end{array}$ & d & 10 & 78 & 32 & 27 & 78 & 70 & 77 & So & 58 & 28 & 41 & 27 & 40 \\
\hline $\begin{array}{l}\text { Sum of Renks - TOTAL } \\
\text { Minimum / best poseibie } 66\end{array}$ & 미 & 232 & 708 & 387 & 378 & 775 & 718 & 784 & 604 & 598 & 344 & 812 & 315 & 280 \\
\hline
\end{tabular}

\begin{tabular}{|c|c|c|c|c|c|c|c|c|c|c|c|c|c|c|}
\hline & Ran & FT Tot & हTन & FT 20 & $\begin{array}{l}\text { FT } 80 \times x \\
\text { Energy }\end{array}$ & ART & AR 2E & $A R$ AIC & ACF न्न & ACF 28 & CCF & CCF 28 & $\begin{array}{l}\text { DWT Haaf } \\
\text { (Lovel B) }\end{array}$ & $\begin{array}{l}\text { DWT Haan } \\
\text { (Leve) } \\
\text { Shinnen }\end{array}$ \\
\hline $\begin{array}{l}\text { Sum of Ranks - TOTAL (magnitude as } \\
\text { well as coemelent of variation of the } \\
\text { index) } \\
\text { Minimum / per possible } 132\end{array}$ & 10 & $\operatorname{san}$ & 1498 & 834 & 883 & 1281 & 1374 & 1383 & 787 & 1030 & 862 & 645 & 1208 & 1110 \\
\hline
\end{tabular}

Minimum / ber possible 132 
values from both demand parameter as well as order of delay perspectives. This is primarily on account of their ability to ignore leads/lags between the data profiles. FT and ACF are well established for analysing univariate data with seasonality, but this analysis shows that these are also effective in the context of multiple data series with seasonal characteristics.

The features however, differ in terms of their parameter sensitivity. While ACF features show a marginal change in consistency with the change in parameters from 7 to 28 , the change is significant for the FT features. FT 7 and FT 28 with the first seven and twenty eight frequencies show poor consistency, which improves when more frequencies are used as in the FFT $80 \%$ Energy option, with the highest consistency being for the FT Total option where all frequencies are used in the index computation. The behaviour of FT 7 and FT 28 is on account of non inclusion of relevant rogue frequencies within the first 7 and 28 frequencies used in these options (this risk associated with these options was highlighted earlier). As regards other features such as CCF and time domain analysis, these were found to give slightly lower consistencies than ACF and FT, which is understandable, as both are based on direct comparisons of time series profiles, though CCF has some advantages as it looks at lags/leads in relationships also. The DWT and the AR model features, however, showed the lowest consistencies. For DWT, this is because wavelets, like the time domain, are also not invariant to leads/lags in data profiles. Moreover, the Haar wavelet used is discontinuous and therefore less able to capture the smooth profiles of the variables. The result for the AR model is however surprising, as this approach has been shown to be effective by Ting et al. (2003) and Caiado (2006). This could be due to the larger number of variables used here as well as the significant non-stationarity induced in the data from rogue seasonality.

The sum of ranks based on average index magnitudes and the coefficient of variation are given in Tables 4.5b and 4.5c respectively. As discussed earlier, the best transformation technique and feature is that which yields both the highest index values as well as has the lowest coefficient of variation. However, many feature parameter combinations are seen to be not similarly good or bad on both these criteria. The sum of ranks for both criteria have therefore been aggregated together and presented at the bottom of Table 4.5c. FT is 
seen to be balanced in meeting both criteria for some parameters such as FT Total and FT $80 \%$ energy. This is evident in their lowest total sum of ranks, though individually they do not have the lowest ranks in either criterion. However, other FT parameters such as FT 7 and FT 28 appear to be less adequate. CCF meets both criteria effectively, while ACF, which showed good consistency, was found to be deficient in the coefficient of variation criteria. DWT and AR model based techniques, which were behind other features on the consistency criteria were similarly placed on these criteria also.

Time based indices are both a less valid measure of rogue seasonality intensity as well as a less effective discriminator of rogue seasonalities of different intensities. As discussed earlier, this is because of the lack of alignment of the cyclic profiles of variables in the time domain. Lower validity is evident in Table 4.5a which shows the consistency (measure of validity) of the time domain index to be relatively low for most demand process and order of delay combinations. The lower discrimination effectiveness is evident in Table $4.5 \mathrm{~b}$ with the sum of ranks of the time based index values being relatively higher (indicating a lower index value) for most combinations. Index based on the time domain is therefore less appropriate and useful than other techniques despite having advantages such as ease in computation and interpretation.

Overall, based on all the three criteria, FT and specifically the FT Total feature parameter combination was judged to be the best for rogue seasonality detection. Using fewer numbers of parameters as in FT 7 and FT 28, though improving efficiency in computation, was seen to reduce accuracy and effectiveness of detection significantly. Intermediate approaches such as FT $80 \%$ Energy therefore seem a good compromise. Index values based on ACF features showed good consistency to varying demand and delay characteristics as well as discrimination ability in view of their large index magnitudes. However, their coefficient of variation was greater relative to other techniques suggesting lower robustness to stochastic variability. With regard to $\mathrm{CCF}$, index values calculated from its features were not very consistent, though it performed adequately on the other two criteria. Finally, the AR model and the DWT were found to be the worst in each of the three criteria. In fact, for some AR model and DWT parameters, the performance on the criteria was worse than even that for the time domain. 


\subsubsection{Comparison of alternative index definitions}

The index values based on different feature parameter combinations for different systems (demand processes and parameters, delay and order of delay) were computed on the basis of the three index definitions individually and correlated with each other. This was done at an individual (stochastic instance) level rather than from averages. Also, the parameter options within each feature were combined together while computing the correlation coefficient for that feature. Table 4.6 gives the correlation coefficient between the alternative index definitions.

\section{Table 4.6 - Correlation coefficient between alternative rogue seasonality indices in a linear three echelon MTS system - All variables used in computation of the indices}

\begin{tabular}{|l|r|r|r|}
\hline & Index and Alt & Index and Alt & Alt 1 and \\
& 1 & 2 & Alt 2 \\
\hline Time & 0.05 & 0.97 & 0.22 \\
FT & 0.96 & 0.95 & 0.85 \\
AR Model & 0.97 & 0.98 & 0.94 \\
ACF & 0.99 & 0.97 & 0.96 \\
CCF & 0.95 & 0.98 & 0.94 \\
DWT & 0.28 & 0.76 & 0.29 \\
\hline \multicolumn{2}{|c|}{ Highlighted indicates low correlation (considered bad) }
\end{tabular}

Table 4.6 shows that for most of the features (FT, AR Model, ACF, CCF), correlation between the alternative rogue seasonality index definitions is high implying that indices derived in these domains are robust and less sensitive to the nature of dissimilarity measurement between CONS and the other variables: minimum or average or in terms of standard deviation. This does not hold for indices derived in the time domain and using DWT coefficients (correlation is low for certain combinations). One reason for this is that the index values in these domains are quite low and therefore relative dispersion between alternative indices is more than that in the other domains.

\subsubsection{Case II: Exogenous demand with seasonality and all variables used}

Exogenous demand with seasonality was considered in order make the analysis more realistic. Two separate analyses, one with sinusoidal cyclicality of frequency 0.01 and the other with frequency 0.09 (unit amplitude in both cases) were carried out. The seasonality was added to the stochastic demand process before the analysis, with the analysis being 
of a similar nature as in the previous section. Frequency of 0.01 was within the amplification range and 0.09 beyond the amplification range, that is, in the attenuation range for all the systems considered (the ranges are determined by delay or $T_{p}$ ). In this way, such contrasting situations that could be encountered in practice were covered.

The spectra plots of system variables for this case revealed transmission of the exogenous seasonality to other variables/echelons at the same frequency. While the upstream system variables in the 0.01 frequency case showed amplification, they were attenuated in the 0.09 frequency case. Rogue seasonality generated endogenously by the system was visible, though, significantly masked for the system with amplification. The nature of the variable profiles therefore seem to depend on the frequency of the exogenous seasonality in relation to the characteristic amplification/attenuation frequency range of the system, and this has the potential to corrupt the index definition logic. The index may not give an accurate indication of rogue seasonality intensity in such cases, and such cases are quite common as system structure is generally unknown and exogenous seasonality strong.

A better approach would be to filter out the exogenous seasonality from all variables before calculating the rogue seasonality index. This is possible since exogenous seasonality is transmitted upstream to many variables, and can therefore by identified on that basis. Therefore, in a test system whose rogue seasonality is being assessed, if many variables across multi echelons including exogenous demand have high signal energy at a particular frequency, this implies that the seasonality is exogenous in nature. Using a notch filter, a well known filtering technique in signal processing (Orfanidis, 2002), this common frequency can be filtered out from all the variables, whereafter the index could be calculated in the usual way based on any feature parameter combination. This approach was tested for a few feature parameter combinations (those found most appropriate in the earlier analysis) and found it to be effective. Figure 4.4 gives the scatter plot between index values without seasonality and with seasonality and notch filtering for seasonalities of 0.01 and 0.09 . The dispersion in the index values for each of the three feature parameter combinations considered is low which demonstrates the effectiveness of the notch filtering based approach. The index is therefore an effective measure for rogue seasonality even for cases where exogenous demand has seasonality; however, 
No seasonality and

Seasonality with

frequency .01

\section{FFT Total}
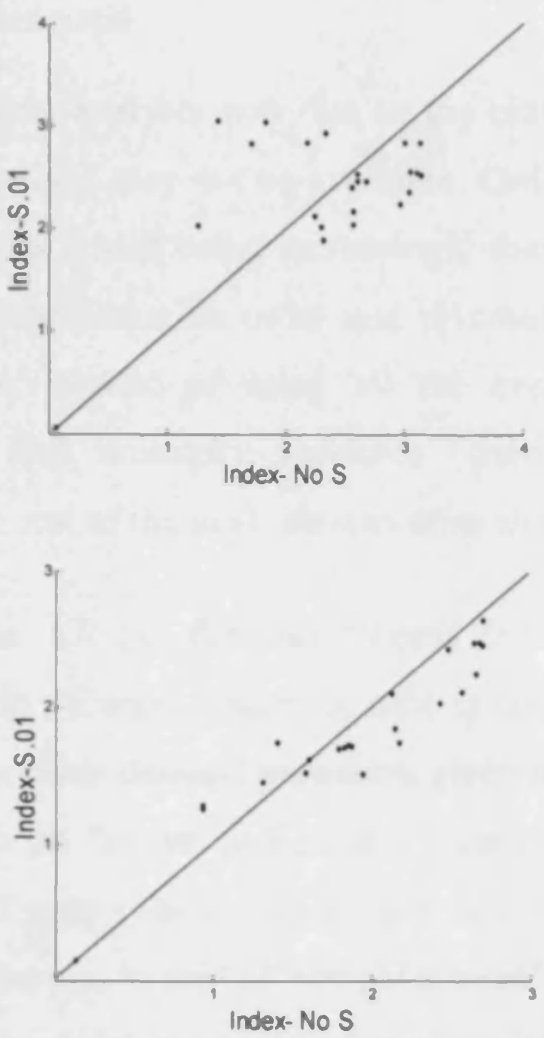

\section{ACF 28}

\section{CCF 28}
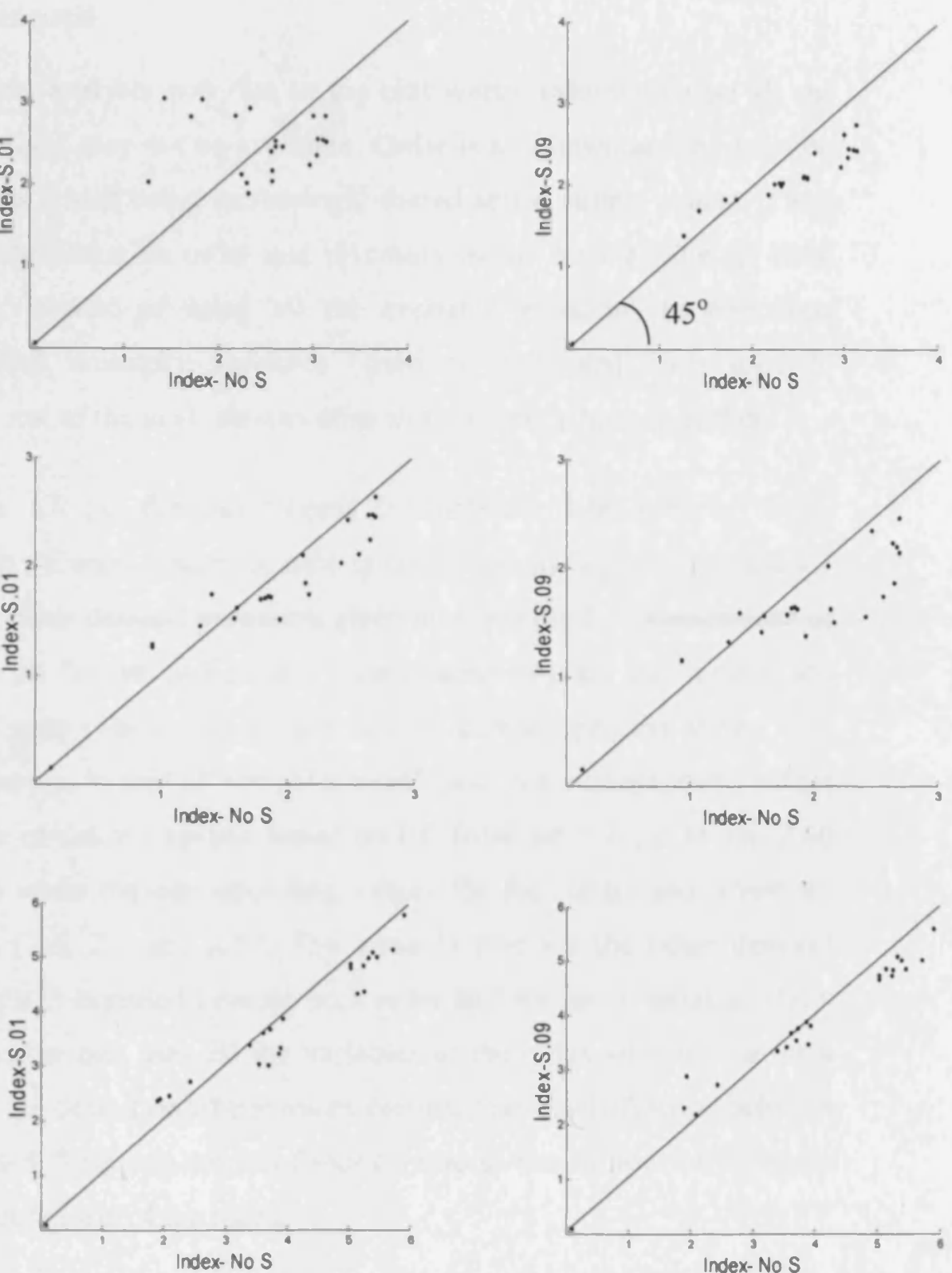

3 echelon MTS system; All demand processes and parameters; Delay order one and infinity; Lead time $(\mathrm{Tp})=3$; Index computed from all variables is used

Figure 4.4 Variation in rogue seasonality index from exogenous demand having an additional seasonal component (seasonal component is notch filtered before index computation) 
prior notch filtering is required to filter out the exogenous seasonality.

\subsubsection{Case III: Exogenous demand without seasonality and only order and inventory variables used}

The rationale for doing this analysis was that in the real world, information on all the variables as considered earlier, may not be available. Order is a rudimentary information while inventory information is also being increasingly shared across supply chains. It was therefore assumed that information on order and inventory would be available for most supply chains. Therefore, instead of using all the available variables, as was done previously, only order and inventory variables (from all echelons) were used in computing the index. The rest of the analysis was done in a similar fashion as earlier.

The index values for the AR (1) demand process for different delay-order of delay combinations and based on different feature-parameter combinations is given in Table 4.7 with the same analysis for other demand processes given in Appendix F. A comparison of the index values between an "all variables used" case (Table 4.4) and the "orders and inventory variables used" case (Table 4.7) for the AR (1) demand process shows only small differences. For example, in the "all variables used" case, the average index values for delay $\left(T_{p}\right) 3$ and order of delay 1 system based on FT Total are 1.71, 2.34 and 2.60 (for $\rho-0.8,-0.5$ and 0.1 ) while the corresponding values for the "order and inventory variables used" case are 1.46, 2.1 and 2.52. The same is true for the other demand processes also. Such a result is expected because both order and inventory variables show rogue seasonality and whether one uses all the variables in the index computation or a subset of these variables, the definition of the index ensures that the difference between their index values is limited. This invariance of index value to the number of variables used also strengthens the definition of the index.

Tables 4.8a, $\mathrm{b}$ and $\mathrm{c}$ give the evaluation of different feature parameter combinations on the basis of consistency, magnitude of index (discrimination) and coefficient of variation of the index (stochastic robustness) where only order and inventory variables were used. Similar to the "all variables used" case (Tables $4.5 \mathrm{a}, \mathrm{b}, \mathrm{c}$ ), FT (specifically FT Total) feature parameter combination is seen to be the most appropriate for rogue seasonality 
Table 4.7 - Rogue seasonality index of a three echelon MTS system based on different feature parameter combinations;

Demand process: AR (1); Only order and inventory variables used

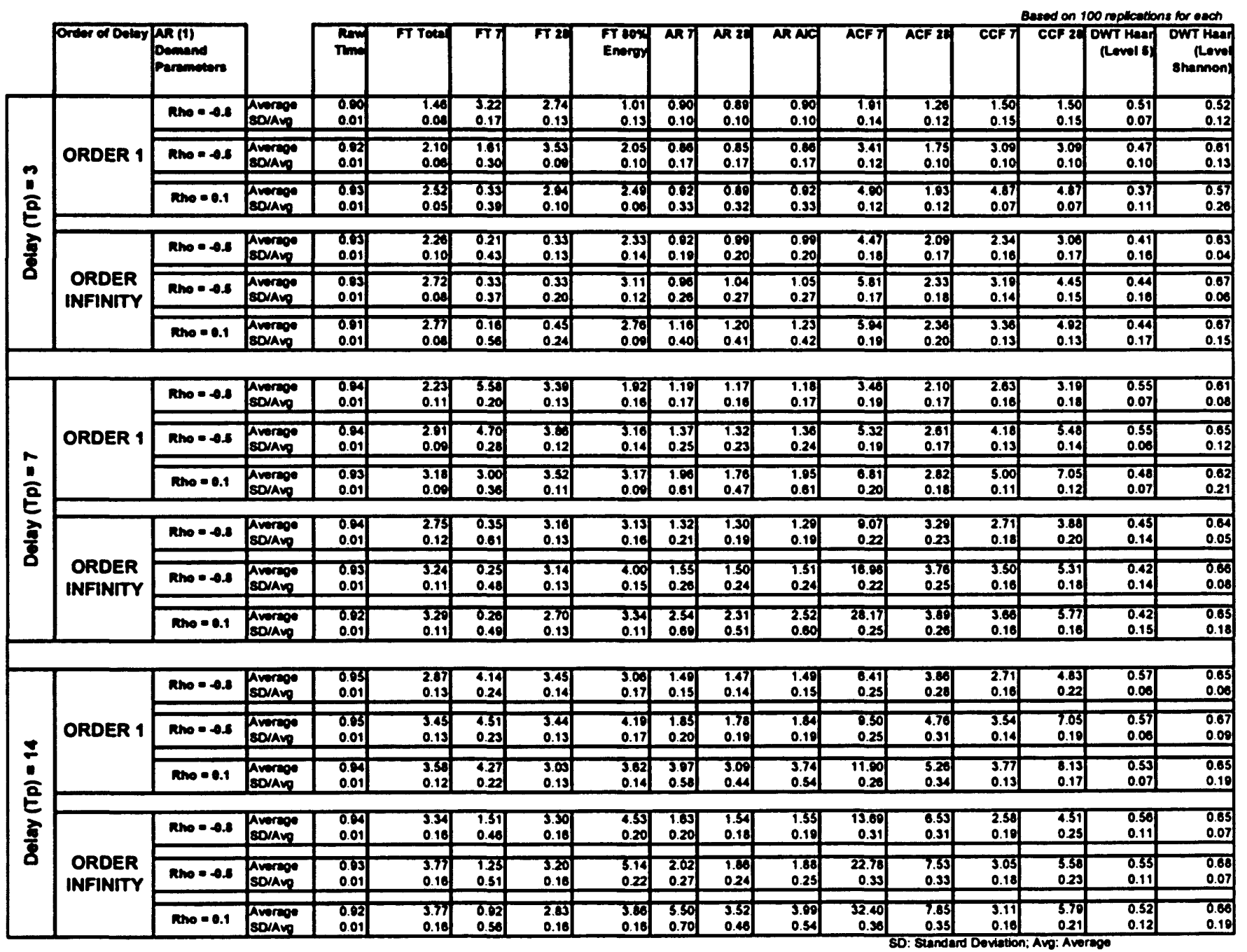

index computation followed by ACF with AR and DWT features at the bottom. A similar rationale, as discussed in the previous paragraph applies here for this observation. Finally, Table 4.9 gives the correlation coefficient between the alternative index definitions where again the results are similar to that the "all variables used" case i.e. high correlation coefficient for the FT, AR model, ACF and CCF features and low correlation coefficient for the time domain and DWT coefficients. 
Table 4.8: Structured evaluation of feature parameter combinations used for rogue seasonality index computation in a three echelon MTS system; Only order and inventory variables used

Table 4.8a

Highlighted indicates best

Consistency of rogue seasonality index for different demand processes and order of delays (Number of cases)

\begin{tabular}{|c|c|c|c|c|c|c|c|c|c|c|c|c|c|c|c|}
\hline $\begin{array}{l}\text { Merture of } \\
\text { Damand } \\
\text { Procoss: }\end{array}$ & Consistoney Basis & $\begin{array}{l}\text { Ram } \\
\text { Time }\end{array}$ & FT Total & FTन & FT 28 & $\begin{array}{l}\text { FT 80\% } \\
\text { Energy }\end{array}$ & AR? & AR 28 & $\overline{\text { AR NCC }}$ & ACF $T$ & ACF 28 & CCF $T$ & CCF 2ब & $\begin{array}{l}\text { DWT Haar } \\
\text { (Lovol of) }\end{array}$ & $\begin{array}{l}\text { DWr Haan } \\
\text { (Lovo) } \\
\text { Shannon) }\end{array}$ \\
\hline \multirow{2}{*}{ AR 1} & Dem and parameters & $3 / 12$ & $11 / 12$ & $3 / 12$ & $3 / 12$ & $8 / 12$ & $11 / 12$ & $11 / 12$ & $11 / 12$ & $12 / 12$ & $12 / 12$ & $12 / 2$ & $12 / 12$ & $2 / 12$ & $7 / 12$ \\
\hline & Order of colay & $2 / 9$ & $9 / 9$ & का & or & ovg & जी & ज्ञा & \$月: & $9 / 9$ & set & $3 / 9$ & $4 / 9$ & $1 / 9$ & प्रा10 \\
\hline \multirow{2}{*}{ MA 1} & Demand perameters & $6 / 12$ & 12112 & ब/12 & $6 / 12$ & 812 & $7 / 12$ & $6 / 12$ & $6 \pi 12$ & $12 / 12$ & $12 / 12$ & $11 / 42$ & 12याय & $3 / 12$ & $10 / 12$ \\
\hline & Order of delay & $3 / 9$ & का & arg & $2 / 9$ & 20 & $9 / 9$ & $9 / 9$ & $8 / 9$ & 99 & 09 & $4 / 9$ & $6 / 9$ & $4 / 9$ & 90 \\
\hline \multirow[b]{2}{*}{ AR 2} & Domend peramelers & 0,6 & $6 / 6$ & $3 / 6$ & $3 / 6$ & 6ब & $3 / 6$ & $3 / 6$ & $3 / 6$ & $6 / 6$ & $6 / 6$ & $5 / 6$ & हत्ब & $1 / 6$ & बहत \\
\hline & Order of delay & 416 & $6 / 6$ & $1 / 6$ & $2 / 6$ & $6 / 6$ & $4 / 6$ & $4 / 6$ & $4 / 6$ & $5 / 6$ & $6 / 6$ & 216 & $4 / 6$ & $4 / 6$ & 60 \\
\hline \multirow{2}{*}{ MA 2} & Domand perameters & $0 / 6$ & $6 / 6$ & $1 / 2$ & कब & $4 / 6$ & $2 / 6$ & $0 / 6$ & 0,6 & हान & $6 / 6$ & $6 / 6$ & 8 酷 & $0 / 6$ & $0 / 6$ \\
\hline & Order of delay & $2 / 6$ & $4 / 6$ & $\infty$ & 0 & $4 / 6$ & $8: \frac{1}{0}$ & 6/: & 6,8 & $5 / 6$ & Bile & $1 / 6$ & $2 / 6$ & $1 / 6$ & $6 \%$ \\
\hline \multirow[t]{2}{*}{ Gaussian } & Order of delay & $0 / 3$ & उ/3 & $0 / 3$ & $0 / 3$ & $3 / 3$ & $3 / 3$ & $3 / 3$ & $3 / 3$ & $3 / 3$ & $3 \mathrm{~s}$ & $0 / 3$ & $1 / 3$ & $1 / 3$ & $3 / 3$ \\
\hline & $\begin{array}{l}\text { Overall Consistency : } \\
\text { Domand and Order of } \\
\text { delay }\end{array}$ & 20/68 & Ext & 12180 & 18Neg & 67/68 & SaNe? & 51/60 & 80/68 & 67/69 & 69/69 & 4Neg | & 53/89 & 17/82 & $66 / 69$ \\
\hline
\end{tabular}

\section{Table $4.8 b$}

Rank analysis based on the magnitude of rogue seasonality index

(For assessing the discrimination ability of the index derived from different feature parameter combinations)

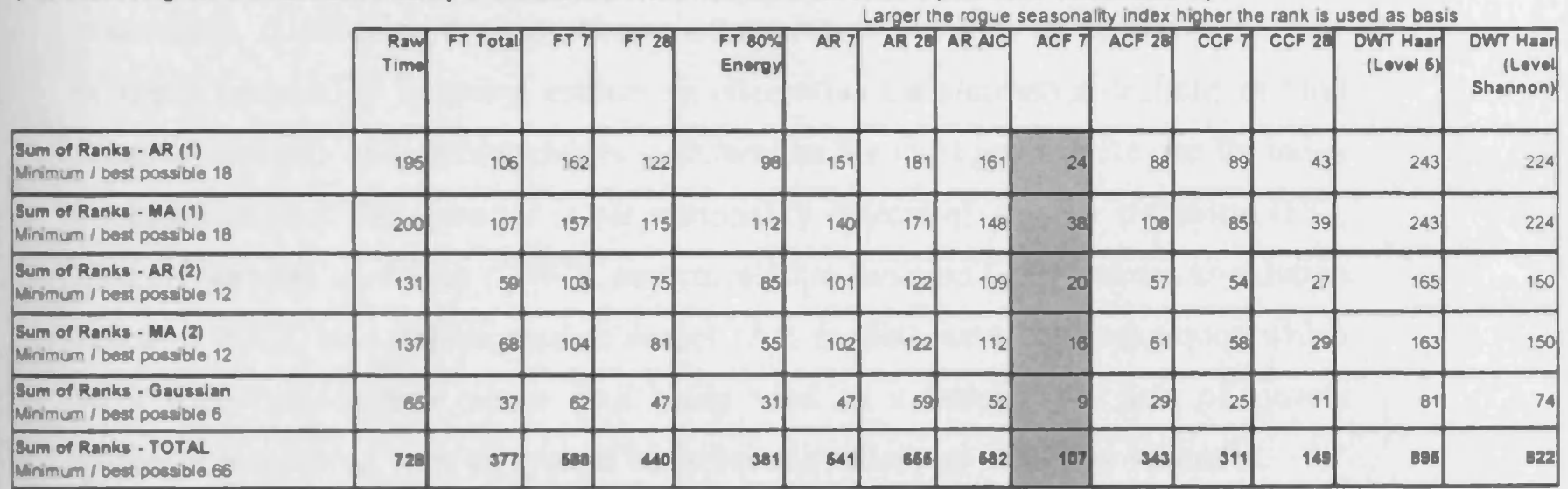

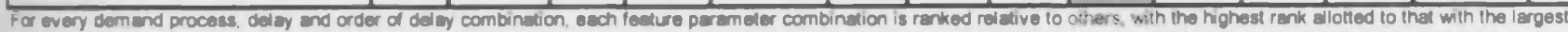

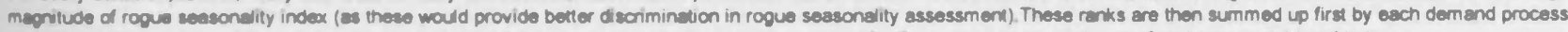
wech a AR (1). MA (1) and then all together to denve a total sum of ranks, Feature parameter combinations with a lower total sum of ranks are considered better

\section{Table 4.8c}

Rank analysis based on the coefficient of variation of rogue seasonality index

(For assessing the stochastic stability of the index derived from different feature parameter combinations)

\begin{tabular}{|c|c|c|c|c|c|c|c|c|c|c|c|c|c|c|}
\hline \multirow{2}{*}{ 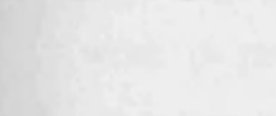 } & & & & & & & \multicolumn{8}{|c|}{ Lower the coefficient of variation the higher the rank is used as basis } \\
\hline & $\begin{array}{l}\text { Ran } \\
\text { Time }\end{array}$ & FT Total & FTन & FT 2E & $\begin{array}{l}\text { FT 80\% } \\
\text { Enorgy }\end{array}$ & ART & AR 2D & ARAC & ACF T & ACF 2E & CCF & CCF 2E & $\begin{array}{l}\text { DWT Hant } \\
\text { (Lovol o) }\end{array}$ & $\begin{array}{l}\text { DWT Han } \\
\text { (Love) } \\
\text { Shannon) }\end{array}$ \\
\hline $\begin{array}{l}\text { Sum of Ranks - AR (I) } \\
\text { Minimum / beext poselble } 18\end{array}$ & 18 & 58 & 236 & 94 & 108 & 203 & 178 & 197 & 188 & 186 & 117 & 147] & 68 & 91 \\
\hline $\begin{array}{l}\text { Sum of Ranks - MA (1) } \\
\text { Minimum / best possible } 18\end{array}$ & 18. & 54 & 228 & 110 & 94 & 222 & 192 & 214 & 177) & 180 & 100 & 127] & 72 & 101 \\
\hline $\begin{array}{l}\text { Sum of Ranks - AR (2) } \\
\text { Minimum I beal poseible } 12\end{array}$ & 12. & 38 & 137 & 68 & 73 & 149 & 130 & 144 & 124] & 117 & 63] & 84 & 55 & 64 \\
\hline \begin{tabular}{|l} 
Rum of Ranks - MA (2) \\
Minimum I best posabible 12
\end{tabular} & 12 & 41 & 160 & 70 & 70 & 134 & 119 & 125 & 131] & 125 & 68 & 90 & 49 & 63 \\
\hline $\begin{array}{l}\text { Sum of Ranks - Goussian } \\
\text { Minimum / besl poserble } 6\end{array}$ & d. & 16 & 73 & 30 . & 24] & 80 & 69 & 76 & 57. & 56 & 31 & 38 & 25 & 48 \\
\hline $\begin{array}{l}\text { Sum of Ranks - TOTAL } \\
\text { Minimum I Dest possible } 68\end{array}$ & 미 & 207 & 832 & 372 & sed & 788 & 508 & 768 & 877 & 664 & 380 & 486 & 269 & 367 \\
\hline
\end{tabular}

\begin{tabular}{|c|c|c|c|c|c|c|c|c|c|c|c|c|c|c|}
\hline & $\begin{array}{l}\text { Ram } \\
\text { Time }\end{array}$ & PT Total & FTr & FT 21 & $\begin{array}{l}\text { FT rox } \\
\text { Enorgy }\end{array}$ & AR T & AR 2E & AR NC & $\overline{A C F} 7$ & ACF 2A & $\overline{\text { CCF } \eta}$ & CCF 2E & $\begin{array}{l}\text { DWT Has } \\
\text { (Lovel B) }\end{array}$ & $\begin{array}{l}\text { DWT Haan } \\
\text { (Lovo) } \\
\text { Shinnon) }\end{array}$ \\
\hline $\begin{array}{l}\text { Sum of Ranks - TOTAL. (magnitude as } \\
\text { will as cooftheiont of variation of the } \\
\text { index) }\end{array}$ & 794 & sat & 1420 & 812 & 750 & 1329 & 1341 & 1338 & 784 & 1007 & 691 & 636 & 1184 & 118 \\
\hline
\end{tabular}




\section{Table 4.9 - Correlation coefficient between alternative rogue seasonality indices in a linear three echelon MTS system - Only orders and inventory variables used}

\begin{tabular}{|l|r|r|r|}
\hline & $\begin{array}{r}\text { Index and } \\
\text { Alt 1 }\end{array}$ & $\begin{array}{r}\text { Index and } \\
\text { Alt 2 }\end{array}$ & $\begin{array}{r}\text { Alt 1 and } \\
\text { Alt 2 }\end{array}$ \\
\hline Time & 0.20 & 0.92 & 0.22 \\
FT & 0.98 & 0.99 & 0.95 \\
AR Model & 0.99 & 0.97 & 0.96 \\
ACF & 1.00 & 0.98 & 0.98 \\
CCF & 0.97 & 0.98 & 0.96 \\
DWT & 0.03 & 0.38 & 0.19 \\
\hline
\end{tabular}

Highlighted indicales low correlation (considered bad)

\subsection{Conclusions}

This chapter investigated rogue seasonality detection in a linear supply chain system to answer Research Question one. This required determining the signature for rogue seasonality, quantifying the signature to determine an index as an objective measure of rogue seasonality intensity, evaluating alternative transformation techniques (and features) and sets of system variables to determine the most appropriate one for index computation (and therefore for rogue seasonality detection). Fourier transform (FT), discrete wavelet transform (DWT), autocorrelation function (ACF), cross correlation function (CCF) and autoregressive model (AR model) were the techniques which were tried besides time series data being used as a benchmark. Sets of system variables considered were all system variables and order and inventory variables.

The signature of rogue seasonality was determined on the basis of the clustering profile of the supply chain variables, specifically whether exogenous demand did or did not get clustered with the other internal operating variables such as inventory and work in process. Rogue seasonality was considered present if the latter was true, otherwise not. To overcome the subjectivity involved in assessing the signature from the cluster profiles, an objective measure called the rogue seasonality index was defined which is based on comparing the dissimilarities amongst the variables in relation to the dissimilarity between these variables and the exogenous demand. It was found to be a robust and accurate indicator of rogue seasonality intensity in a supply chain. For example, the index value for a supply chain which does not show rogue seasonality characteristics (make to order or MTO) is 0 while it is greater than 0 for systems with rogue seasonality (make to stock or MTS). Also, greater intensity of rogue seasonality in the supply chain gets reflected as a higher index value. Though three definitions of index were tried, a high correlation between the index values 
based on these definitions indicated that they were essentially measuring the same thing and any of them could be used. The index was also evaluated with different sets and number of variables (all variables, only orders and inventory variables), where again the index values did not change substantially, and were able to indicate the rogue seasonality intensity accurately. The index is therefore also robust to choice of variables as long as most of the variables exhibited the same type of behaviour (rogue cyclicality or no rogue cyclicality). In any case, use of multiple variables in the computation of the index makes it less susceptible to inconsistencies in some variable profiles. Finally, the index definition is applicable even in the case of exogenous demand having seasonality; exogenous seasonality is required to be identified on the basis of its common presence in demand and other variable profiles and filtered before computation of the index.

Compared to other features, the FT feature was found to be better for computing the index (and therefore for rogue seasonality detection), with index values derived from it showing high consistency, discrimination ability and stochastic stability. FT has been shown to be effective on a generic basis for individual time series by Agrawal et al. (1993), Caiado et al. (2006) and Vlachos et al. (2006). This study shows that it can also be applied to multivariate data as well as data with inconsistent periodicity and thus validates the findings of Thornhill and Naim (2006). Parameters used, which for FT is the number of frequency channels, were found to significantly impact the index values and hence effectiveness of detection. FT Total which uses features from all the frequency channels was found to perform better than options such as FT 7 and FT 28 which use a lesser number of frequencies. However, FT 80\% Energy option was close to FT Total in terms of all the assessment criteria used and could be used instead of the latter for computing the index. Regarding other features, ACF was a close second to FT in terms of rogue seasonality detection. DWT and AR model features on the other hand were found to be the least effective. The findings for DWT are surprising, as the multi resolution property of wavelets was expected to yield better results than FT. With regard to the AR model based approach, it can be concluded that it is less effective for data with cyclical characteristics.

In the next chapter a more realistic context is used to validate the answers to research questions obtained in this chapter. 


\section{Chapter 5 - Analysis of rogue seasonality in a non-linear supply chain system}

The previous chapter used the context of a linear supply chain to answer research question one. Specifically, signature and index for detecting the rogue seasonality and assessing its intensity were proposed and tested, together with the best time series transformation technique (feature and its parameter) to be used to derive them. In this chapter, answers to the research question obtained from chapter one will be evaluated using a non-linear supply chain context. Specifically, the Beer game (Sterman, 1989) supply chain system is used. This is because it has been used by many researchers to study the Bullwhip effect (see sections 2.3.1.1, 2.3.1.2 and 3.6.1.4) and therefore serves as a benchmark for comparison purposes. This system allows non-linearities such as backlogs and batching to be incorporated so that their impact on rogue seasonality generation and detection (signature and index) could be assessed. Here, the system is also used to assess the impact of alternative control systems and other variables such as shipment on the rogue seasonality dynamics. The rationale for analyzing these is explained below:

a) Backlogs: Backlogs are cumulative pending deliveries because of non availability of stock in earlier periods and assume that customers are willing to wait for these deliveries. Backlogs cause non linearities in system operations (Sterman, 2000) and they can be observed in many sectors.

b) Batching: Aggregation of orders or batching is a common practice because of economies of scale in production/transport, use of MRP systems, economic order quantity (EOQ) based ordering and economic production quantity (EPQ) based production. Though the impact of batching has been studied by researchers such as Cachon (1999), Kelle and Milne (1999), Holland and Sodhi (2004), Potter and Disney (2006) and Riddalls and Bennett (2001), their focus was on the Bullwhip effect. Here we are considering the impact of batching on rogue seasonality which has not been considered before. 
c) Hybrid (MTO-MTS) control system: The hybrid MTO-MTS system is considered to be more widely used than pure MTO/MTS systems by researchers such as Anderson Jr et al (2005). The impact of the hybrid system was therefore assessed.

d) Shipment: Shipment is also a common system variable like order and inventory, (Lee and Whang, 2000). Moreover, its inclusion in the model structure ensures a more realistic bi-directional (both upstream and downstream) propagation of the dynamics (Shukla and Naim, 2009).

This chapter is structured as follows. First, the Beer game supply chain system is described. Next, I explain the control theory or FR analysis of a single echelon in this system (linear representation i.e. without backlogs). Subsequently, development of the system dynamics model of the total Beer game system is explained, together with generation of rogue seasonality (in this system) and the effectiveness of the signature and index for its detection. Finally, detailed simulation (multiple replications) with different stochastic demand processes, lead times, ordering policies, batch sizes for rogue seasonality generation and different feature parameter combinations with alternative index definitions for rogue seasonality detection is used to identify the most appropriate feature for index computation (and therefore rogue seasonality detection).

\subsection{Description of the non- linear (Beer game) supply chain system}

The Beer game supply chain system used in this study mimics the ordering and production decisions of a four echelon supply chain (Retailer R, Wholesaler W, Distributor D and Factory F) with flow of orders upstream from the Retailer through to the Factory and flow of material or shipments downstream as shown in Figure 5.1.

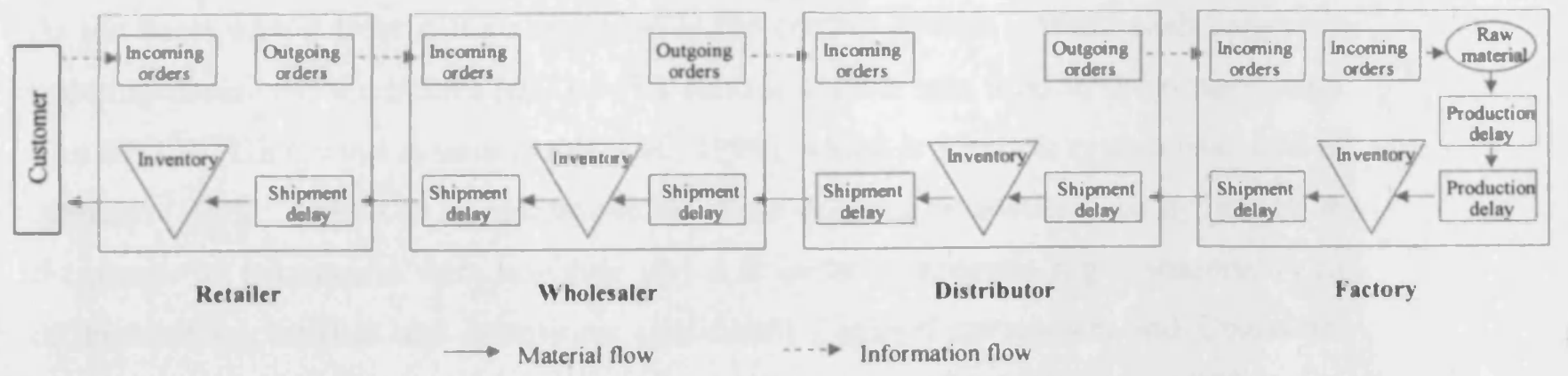

Figure 5.1 Schematic of the Beer game supply chain system (Sterman, 1989) 
The Beer game supply chain system operates as follows. The Retailer receives the customer's demand, it ships the quantity demanded to the customer and then decides how much to order from its upstream echelon or Wholesaler. This procedure then gets repeated at each echelon with placement of orders to the immediate supplier to fulfill the expected demand from the immediate customer. It is assumed that in the case of the supplier being unable to meet demand in certain periods due to occurrence of stock out, a backlog is created which the supplier is required to service in later periods. With the goal of the system being to minimize costs on a cumulative basis, and penalties applied for carrying both excess inventory as well as backlogs, ordering decision making is difficult. This difficulty is further compounded from delays in the system; order information and shipment delays between echelons ( 2 time periods each) and production delay ( 3 time periods) at the factory, the delays being of the pipeline kind (delay of order infinity). In the simulated version of the game, the player is replaced by an ordering heuristic at each echelon. This is explained in a subsequent section.

The Beer game was originally used to demonstrate the behavioural cause of the Bullwhip effect where the role playing version of the game was used (see Section 2.3.1). However recently, this game has also been simulated by many researchers, primarily to understand and improve its dynamic behaviour (see Table 3.3). In the context of rogue seasonality investigation, though any structure or system could be used for simulation, use of common structures/systems permits model validation, comparison of results and hence more effective knowledge building. Thus the Beer game system was considered more appropriate. A further reason (for using this system) was that, despite simplification, it is still reasonably representative of real world supply chains (Larsen et al., 1999).

At the heart of the Beer game simulation is the control system driving each echelon's ordering decisions; the hybrid MTO-MTS control system was used in the present case. The APIOBPCS control system (John et al., 1994), which is a hybrid system was used as Sterman (1989) found it to be representative of the ordering heuristics used by players in the game. Its parameters were however varied in order to generate rogue seasonality of different characteristics and intensities; specifically Optimal parameters and Unoptimal parameters options were used. Parameter values associated with good dynamic 
performance in relation to the Bullwhip effect suggested by John et al. (1994) are referred to as Optimal parameters (see Appendix A). On the other hand in the Unoptimal parameters option, the pipeline feedback term $\left(T_{w}\right)$ is set to infinity (so that there is no pipeline feedback) with all the other parameter values being the same as in the Optimal parameters option. Sterman (1989) observed that lack of pipeline feedback is associated with greater order amplification (Bullwhip effect) and hence Unoptimal parameters is termed as such. Though Optimal and Unoptimal parameters options are defined on the basis of the Bullwhip effect, it was expected that these would generate rogue seasonality of different characteristics in the system (Kim and Springer, 2008).

\subsection{Frequency response analysis of a hybrid MTO-MTS system}

The total Beer game system is not analytically tractable as it is a high order non-linear differential equation (Sterman, 1989). One option is to analyse each echelon in the system individually, and use the insights so obtained to understand the total system behaviour. However, the system considered here is non linear and analytical techniques such as control theory can only handle linear problems. A way to tackle this problem is to ignore the backlogs and treat each echelon as linear; then carry out a similar control theory (FR analysis) as in the previous chapter, but with a hybrid MTO-MTS system and Optimal parameters and Unoptimal parameters options. The transfer functions of variables for individual echelons could then be coupled together to derive them for the total Beer game system (transfer functions as well as FR), which could be compared with the FR's of those variables computed from the Beer game simulation output (in which the impact of backlogs and associated non-linearities is included). Such an analysis would not only help in understanding the role and extent of impact of backlog related non-linearities on system behaviour, but also in validating the Beer game simulation model. This approach was used in this chapter with APIOBPCS being used as ordering system at each echelon. The analysis summary for a single echelon based on linear control theory is given in Table 5.1 which involved the use of Matlab® in the computations (See Appendix $G$ for a sample computation). FR analysis of the whole Beer game system is explained in a subsequent section. Table 5.1 gives the FR values of key system variables (ORATE, COMRATE, AINV, WIP) at critical frequencies for both the Optimal parameters and 
Table 5.1 - Frequency response (FR) functions of variables at select points for hybrid MTO-MTS systems (Order of delay in the systems is considered to be infinity)

\begin{tabular}{|c|c|c|c|c|c|}
\hline & & & \multicolumn{3}{|c|}{ Outputs are given here in terms of angular frequencies (av) } \\
\hline & Transfer functions for different variables & $\begin{array}{l}\text { FR at freq }(\omega)= \\
0 \text { or } \omega_{0}\end{array}$ & $\begin{array}{l}\text { Freq }(\omega) \text { for } \\
\max F R\end{array}$ & Max FR & $\begin{array}{l}\text { Freq }(\omega) \\
\text { at } \mathrm{FR}=1 \text { or } \\
\omega_{\text {Crossover }}\end{array}$ \\
\hline \multirow{4}{*}{ 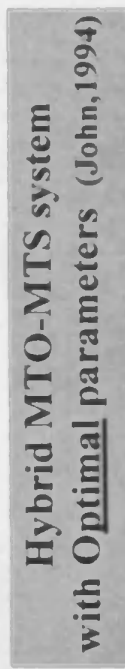 } & $\frac{\text { ORATE }}{\text { CONS }}=\frac{14 T_{p}^{5} s^{5}+4 T_{p}^{4} s^{4}-168 T_{p}^{3} s^{3}-48 T_{p}^{2} s^{2}+2016 T_{p} s+576}{8 T_{p}^{6} s^{6}+12 T_{p}^{5} s^{5}-140 T_{p}^{4} s^{4}+120 T_{p}^{3} s^{3}+672 T_{p}^{2} s^{2}+1440 T_{p}+576}$ & 1 & $\frac{1.014}{T_{p}}$ & 1.688 (constant) & $\frac{2.135}{T_{p}}$ \\
\hline & $\frac{\text { COMRATE }}{C O N S}=\frac{14 T_{p}^{3} s^{3}-80 T_{p}^{2} s^{2}+144 T_{p} s+48}{8 T_{p}^{4} s^{4}+60 T_{p}^{3} s^{3}+124 T_{p}^{2} s^{2}+144 T_{p}+48}$ & 1 & $\frac{1.014}{T_{p}}$ & 1.688 (constant) & $\frac{2.135}{T_{p}}$ \\
\hline & $\frac{W I P}{C O N S}=\frac{6 T_{p}\left(7 T_{p} s+2\right)}{2 T_{p}^{4} s^{4}+15 T_{p}^{3} s^{3}+31 T_{p}^{2} s^{2}+36 T_{p} s+12}$ & $T_{p}$ & $\frac{0.937}{T_{p}}$ & $1.62 * T_{p}$ & $\begin{array}{l}\text { Complex function } \\
\text { of } T_{p}\end{array}$ \\
\hline & $\frac{A I N V}{C O N S}=-1 / 2 \frac{T_{p}^{2}\left(4 T_{p}^{2} s^{3}+23 T_{p} s^{2}+102 s\right)}{2 T_{p}^{4} s^{4}+15 T_{p}^{3} s^{3}+31 T_{p}^{2} s^{2}+36 T_{p} s+12}$ & 0 & $\frac{0.983}{T_{p}}$ & $1.863 * T_{p}$ & $\begin{array}{l}\text { Complex function } \\
\text { of } T_{p}\end{array}$ \\
\hline \multirow{4}{*}{ 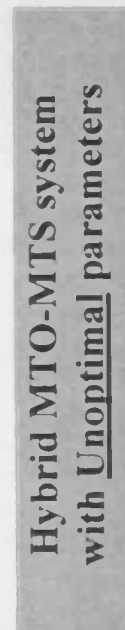 } & $\frac{\text { ORATE }}{\operatorname{CONS}}=\frac{3 T_{p}^{3} s^{3}+19 T_{p}^{2} s^{2}+42 T_{p}+12}{2 T_{p}^{4} s^{4}+15 T_{p}^{3} s^{3}+19 T_{p}^{2} s^{2}+30 T_{p}+12}$ & 1 & $\frac{1.311}{T_{p}}$ & 3.335 (constant) & $\frac{2.208}{T_{p}}$ \\
\hline & $\frac{\text { COMRATE }}{\operatorname{CONS}}=\frac{3 T_{p}^{3} s^{3}-17 T_{p}^{2} s^{2}+30 T_{p} s+12}{2 T_{p}^{4} s^{4}+15 T_{p}^{3} s^{3}+19 T_{p}^{2} s^{2}+30 T_{p} s+12}$ & 1 & $\frac{1.311}{T_{p}}$ & 3.335 (constant) & $\frac{2.208}{T_{p}}$ \\
\hline & $\frac{W I P}{C O N S}=\frac{T_{p}\left(36 T_{p} s+12\right)}{2 T_{p}^{4} s^{4}+15 T_{p}^{3} s^{3}+19 T_{p}^{2} s^{2}+30 T_{p} s+12}$ & $T_{p}$ & $\frac{1.298}{T_{p}}$ & $3.09 * T_{p}$ & $\begin{array}{l}\text { Complex function } \\
\text { of } T_{p}\end{array}$ \\
\hline & $\frac{A I N V}{C O N S}=-\frac{2 T_{p}^{4} s^{3}+12 T_{p}^{3} s^{2}+36 T_{p}^{2} s}{2 T_{p}^{4} s^{4}+15 T_{p}^{3} s^{3}+19 T_{p}^{2} s^{2}+30 T_{p} s+12}$ & 0 & $\frac{1.305}{T_{p}}$ & $3.01 * T_{p}$ & $\begin{array}{l}\text { Complex function } \\
\text { of } T_{p}\end{array}$ \\
\hline
\end{tabular}

Unoptimal parameters: Pipeline feedback is not considered; other parameter values are same as that in John et al. (1994) All profiles are similar: increasing trend, attainment of a peak and decreasing trend thereafter with $w$

Frequency response function has been derived by substituting $j^{*} \omega$ for $s(\omega=$ angular frequency) and taking the magnitude of the resulting complex function 
Unoptimal parameters options. The transfer function expressions have high orders because of use of 2 nd order Pade approximation (Nise, 1995) for pipeline delay. All the variables in both Optimal and Unoptimal parameters systems have similar FR profiles which are increasing-decreasing with an in-between maxima. The FR profile values are also greater than one for a range of frequencies, signifying amplification and rogue seasonality generation in these hybrid MTO-MTS systems. However, the intensity of rogue seasonality generated in the Optimal Parameters option is in between that which is generated in MTO and MTS systems which is reflected in the $\max F R$ values of the variables in these systems. For example, while the max $F R$ value of $O R A T E / C O N S$ for the MTS delay order infinity system is 2.307 (Table 4.1), its value for the hybrid MTOMTS system (Optimal parameters) is 1.688 and 0 for MTO (order of delay infinity system). The same is true for the other analyzed system variables as well. This variation in rogue seasonality intensity can be explained on the basis of the different proportion of exogenous demand (CONS) being used in the ordering decision; CONS is not used at all in an MTS system, used completely in an MTO system and used partially in the hybrid MTO-MTS system. However, other terms in the ordering rule also play a role, which is apparent when the two hybrid system options are compared. The max $F R$ values of all variables in the Unoptimal parameters option are greater than those in Optimal parameters indicating greater rogue seasonality intensity in the former vis-à-vis the latter. This is because of no pipeline damping being applied in the former option.

The analytical FR analysis of hybrid MTO-MTS systems strengthens the hypothesis about rogue seasonality manifestation, which is multiple variables oscillating at a similar frequency with this frequency not being present in CONS. The logic of the signature and index for detecting and measuring rogue seasonality therefore, also applies. However, these judgments are based on the analysis of only a few system variables, a single echelon system and which is also linearly represented, that is, non-linearities from backlogs are ignored. Analysis of the total Beer game system is therefore required, to both assess the propagation of rogue seasonality dynamics in different variables/ echelons as well as the applicability of its signature and index. A system dynamics simulation facilitates such an analysis, and was therefore used. The simulation analysis also helps in 
assessing the impact of multiple rather than a single frequency being amplified and marginal dissimilarities in the FR profiles of variables, on the signature and index of rogue seasonality.

\subsection{System dynamics simulation of the Beer game system}

The system dynamics simulation model of the Beer game supply chain was constructed in Excel with Visual Basic for applications (VBA), the latter for easy visualization of the dynamics (see Figure 3.4). The difference equations used in the model formulation are given in Table 5.2 and are developed as follows. The different exogenous demand processes applied at the Retailer are given in equation $5.1 \mathrm{~b}$ while equations $5.1 \mathrm{c}, 5.2 \mathrm{a}$ and $5.2 \mathrm{~b}$ model the delays in order information and shipments which are of the pipeline kind (delay of order infinity). Inventory and backlogs are modeled separately in equations 5.6 and 5.7 respectively. Shipments are included in the model to take account of nonlinearities associated with the backlog situation. Shipments are set to zero when there is no inventory available, and when inventory does become available, the echelon ships what is ordered plus any backlog that has been accumulated (equations 5.3, 5.4, 5.5). Equation 5.8 gives the computation for exponential smoothing forecasting while the work in process is calculated in equations $5.10 \mathrm{a}$ and $5.10 \mathrm{~b}$. The work in process computation for all except the factory echelon includes three terms: orders that have been placed but not transmitted to the upstream echelon, shipments made by the upstream echelon but not yet received and backlogs. In the case of the factory, the orders placed are received after a fixed production delay and therefore work in process includes just the previous orders placed term. The ordering decision based on APIOBPCS is captured in equation 5.14 with the batching constraints on orders and shipments being applied in equations 5.15 and 5.16. The decision parameters used in the model are lead times (or delays) in order information, shipping and factory production, APIOBPCS parameters $T_{a}, T_{i}$, and $T_{w}$ and batch size $b$.

\subsection{Verification of Beer game simulation and rogue seasonality analysis}

Any simulation model needs to be assessed for correctness (verified) before using it in a 


\section{Table 5.2 - Difference equations used to simulate the Beer game supply chain system}

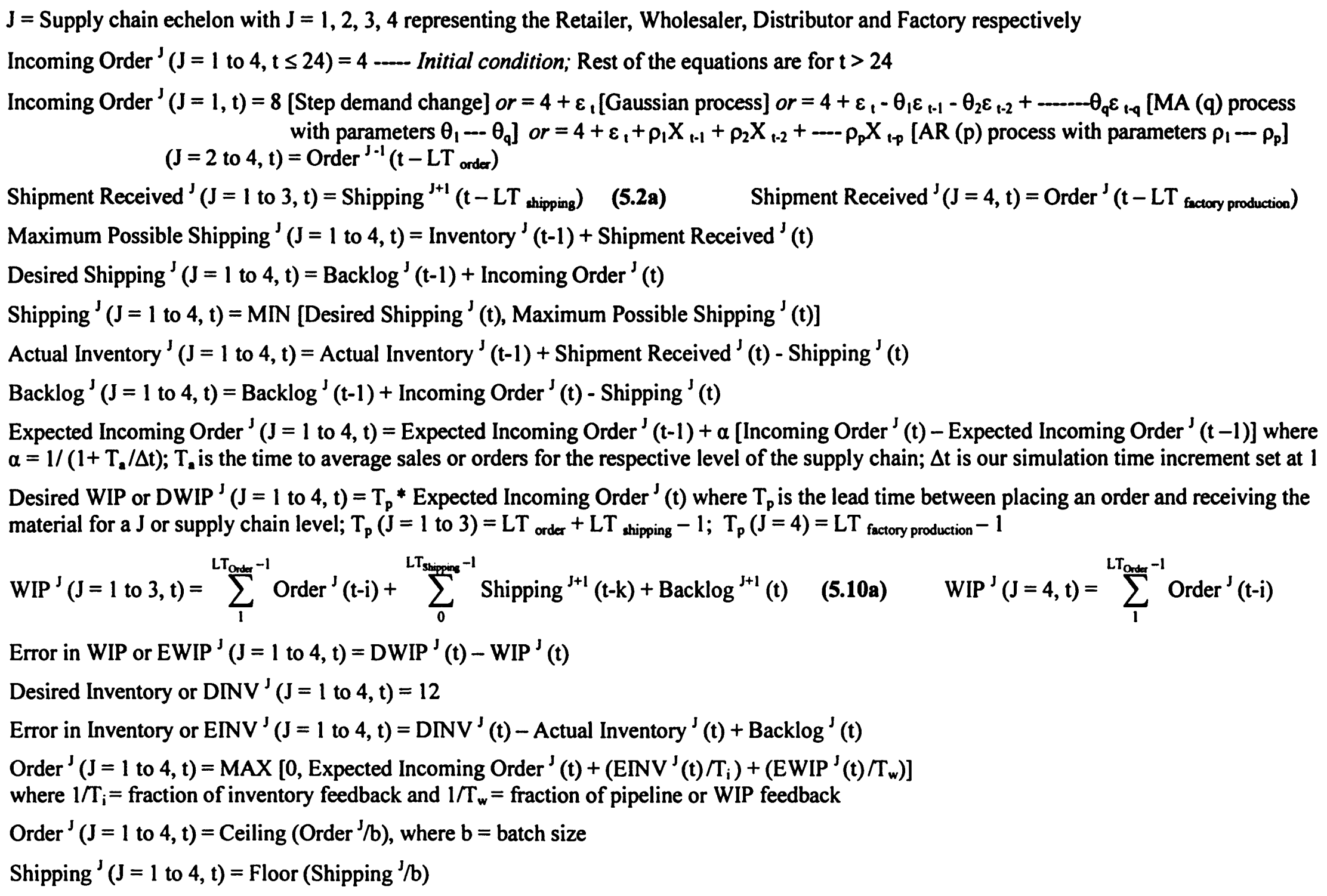

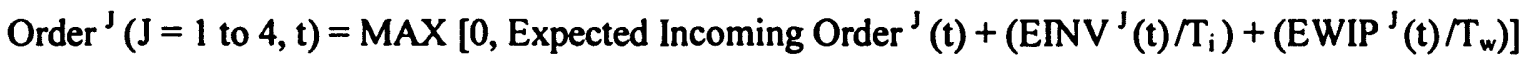

where $1 / T_{i}=$ fraction of inventory feedback and $1 / T_{w}=$ fraction of pipeline or WIP feedback

$\operatorname{Order}^{\mathrm{J}}(\mathrm{J}=1$ to $4, \mathfrak{t})=$ Ceiling (Order $\left.\mathrm{J} / \mathrm{b}\right)$, where $b=$ batch size

Shipping ${ }^{\mathrm{J}}(\mathrm{J}=1$ to $4, \mathrm{t})=$ Floor (Shipping $\left.{ }^{\mathrm{J}} / \mathrm{b}\right)$ 
research context. Two approaches for model verification are used. The first approach involves comparing the FR or Amplitude responses (Amp R) computed from the simulated Beer game output to the analytically derived ones for a linear four echelon system with a similar structure as the Beer game that is same delays and order of delays at different echelons. Select system variables are compared in this way. The second verification approach involves subjecting the simulation to the same conditions as used by Sterman (1989) in his experiment and comparing the profiles of variables obtained (to those obtained by him).

\subsubsection{Verification of Beer game simulation by frequency response (FR) analysis}

The analytically computed FR for a linear representation of the Beer game system is compared to that computed from the simulated Beer game output and this is depicted in Figure 5.2. The analytical FR computation is for a four echelon APIOBPCS with the same delays and order of delays in individual echelons as the Beer game system used. Matlab® is used for the multi echelon computation (see sample in Appendix G) which uses the single echelon analysis given in Table 5.1.

System variables ORATE/CONS, COMRATE/CONS, AINV/CONS and WIP/CONS in each of the four echelons for each of the Optimal parameters and Unoptimal parameters are compared. The Beer game simulation uses lead times of 2 (order information), 2 (shipment) and 3 (production) which are the same as that used by Sterman (1989). Autoregressive of order two (AR 2: $\rho_{1}=0.1 \rho_{2}=-0.8$ ) is used as the demand process, which is because it has low energy in the amplification range frequencies (see Appendix C), which means lower backlogs (and associated non-linearities) and therefore greater clarity in the FR profiles. The FR profiles are averaged over 30 replications for a smoother representation. The analysis of Figure 5.2 reveals the following:

a) All variables in all echelons in the simulated system have FR peaks at the same frequency as suggested by the analytical analysis of the equivalent linear system. For example, the frequency associated with peak FR for Retailer, Wholesaler and Distributor echelons in both the simulated and analytical systems is 0.04 for the Optimal parameters option. Lead time of 4 (order information + shipment) is used in the analytical 

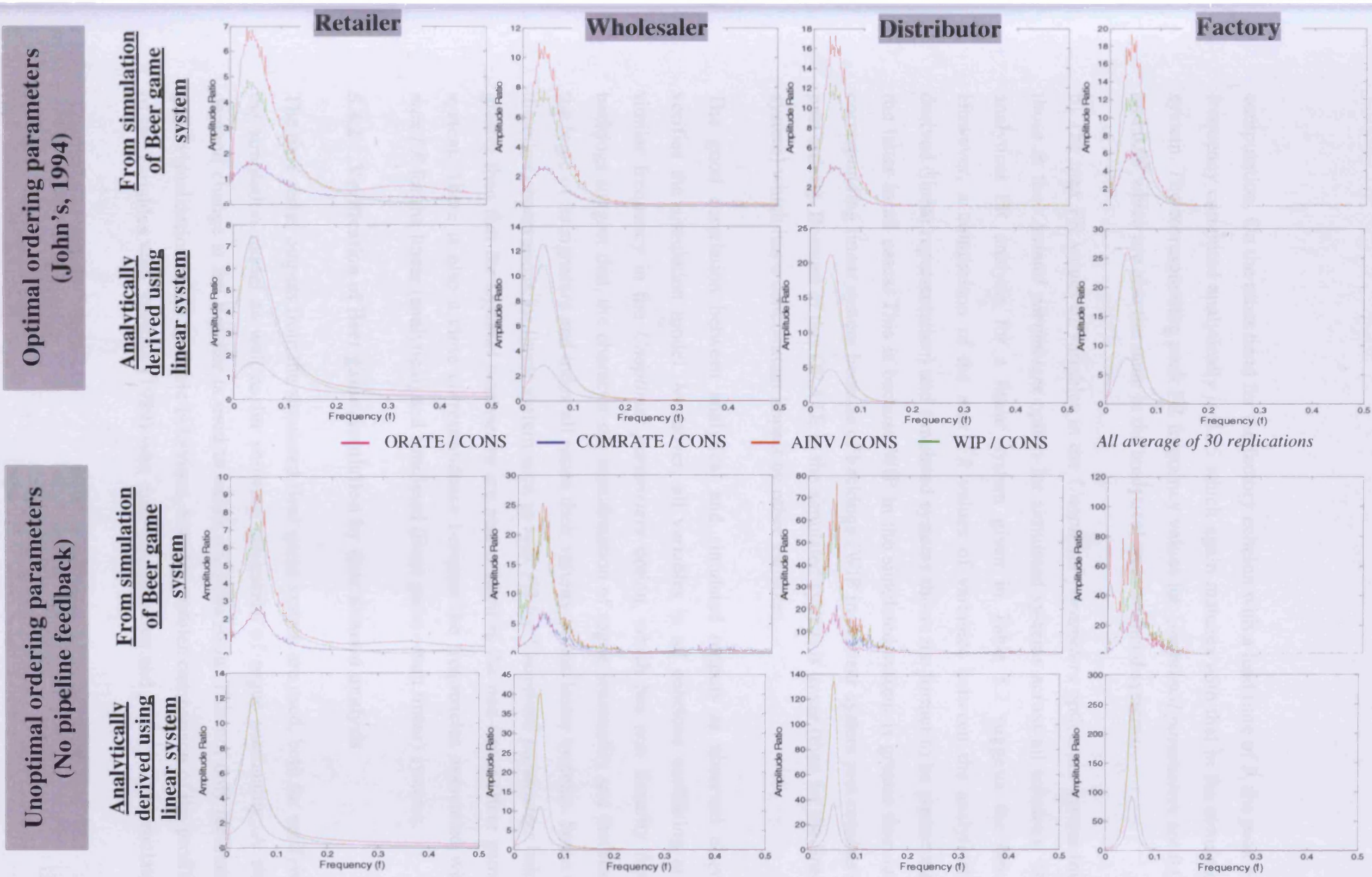

Figure 5.2 Comparison of frequency response derived analytically for a four echelon linear supply chain with that computed from the Beer game simulation output; Demand process used in simulation: AR (2): $\rho_{1}=0.1 \rho_{2}=-0.8$; Lead times: Order 2, shipment 2, production 3 
computation. On the other hand for the factory echelon with a lead time of 3 , the peak FR frequency computed analytically is 0.05 which again matches with that in the simulated system. The corresponding peak FR frequency values for Unoptimal parameters are 0.05 and 0.07 which are also the same in the analytical and simulated systems.

b) The max FR values of variables in the Unoptimal parameters option are greater than those in the Optimal parameters option for simulated systems across all echelons. The analytical FR analysis for a linear system given in Table 5.2 suggests the same. However, a comparison of the max $F R$ values of variables between the analytically derived (linear representation) and simulated systems shows the former to be greater than the latter in all cases. This is because WIP in the simulated system is greater than in a corresponding linear system because of backlogs (WIP in a linear system just consists of past orders). Because of this ORATE in the simulated system is lower (than for the linear system) which has a concomitant impact on other variables.

The good correlation between analytical and simulated outputs as observed above, verifies the simulation model. Moreover, all variables in all echelons oscillating at a similar frequency in the Unoptimal parameters option, which has non linearity from backlogs suggest that, the characteristic manifestation of rogue seasonality and therefore the logic of its signature and index all retain their validity in non linear systems. Some of the other observations for linear system such as max FR for Unoptimal parameters being greater than that for Optimal parameters are also evident in the non-linear (Beer game) system. There is also a close correspondence between the frequencies associated with $\max F R$ for the linear (analytical) and simulated (Beer game - non linear) systems.

\subsubsection{Verification of Beer game simulation by time domain analysis}

The time series outputs from the simulated Beer game system are used, both for verifying the simulation model as well as for showing generation of rogue seasonality. A step demand change at the Retailer is used to excite the simulation. This not only facilitates good visualization of the dynamic behaviour, but also enables comparison of the profiles of the variables with Sterman (1989) who used the same demand pattern. The simulated 
data analysis here uses all the system variables unlike the analytical FR analysis where only select system variables were used. Finally, the impact of batching on the time series profiles of variables is also highlighted. The normalized time series profiles of system variables (36 numbers) for each of Optimal parameters, Unoptimal parameters and Optimal parameters with batching cases are given in Figure 5.3. It is to be noted that the lead times for the batching case at 334 (for ordering, shipment and production) are higher than those for the other cases considered $\left(\begin{array}{lll}2 & 2 & 3\end{array}\right)$. This was done to more effectively highlight the impact of batching on the profiles of variables.

Sterman (1989) depicted order and inventory profiles in his experimentation with the role playing version of the Beer game. Profiles in his study cannot be exactly compared to those generated in the Beer game simulation replicated here. This is because the focus in this case is on rogue seasonality, with variables normalized to enable effective profile comparisons while Sterman's focus was on amplification or Bullwhip effect. However, the profiles of variables in the two cases, still have some visible similarities. A sudden jump in exogenous demand at the retailer creates a backlog situation due to system delays in ordering and receiving material, which in turn increases the tendency to over order on account of feedback loops. Orders and backlogs therefore get increasingly amplified in upstream echelons. However, over time, this gets reversed as the distorted order information manifests itself as increased material receipts, causing both excess inventory as well as dampening future orders. An oscillating profile is thus created which is evident in the order (variable numbers 9, 18, 27 and 36) and net inventory profiles (variable numbers 4, 13, 22 and 31) of all echelons. Shipping profiles which depend on order as well as backlog profiles show a similarly oscillating profile but with attenuation from factory to the retailer (called the backlash effect by Shukla et al (2009)). Other variables such as orders received and shipments received are delayed versions of the order and shipping variables and hence have similar oscillatory characteristics. Hence, most variables (except forecast and desired WIP) show oscillatory characteristics, though the exogenous demand (step) did not have the same, indicating rogue seasonality is generated 


\section{LT 223}

Optimal parameters No Batching

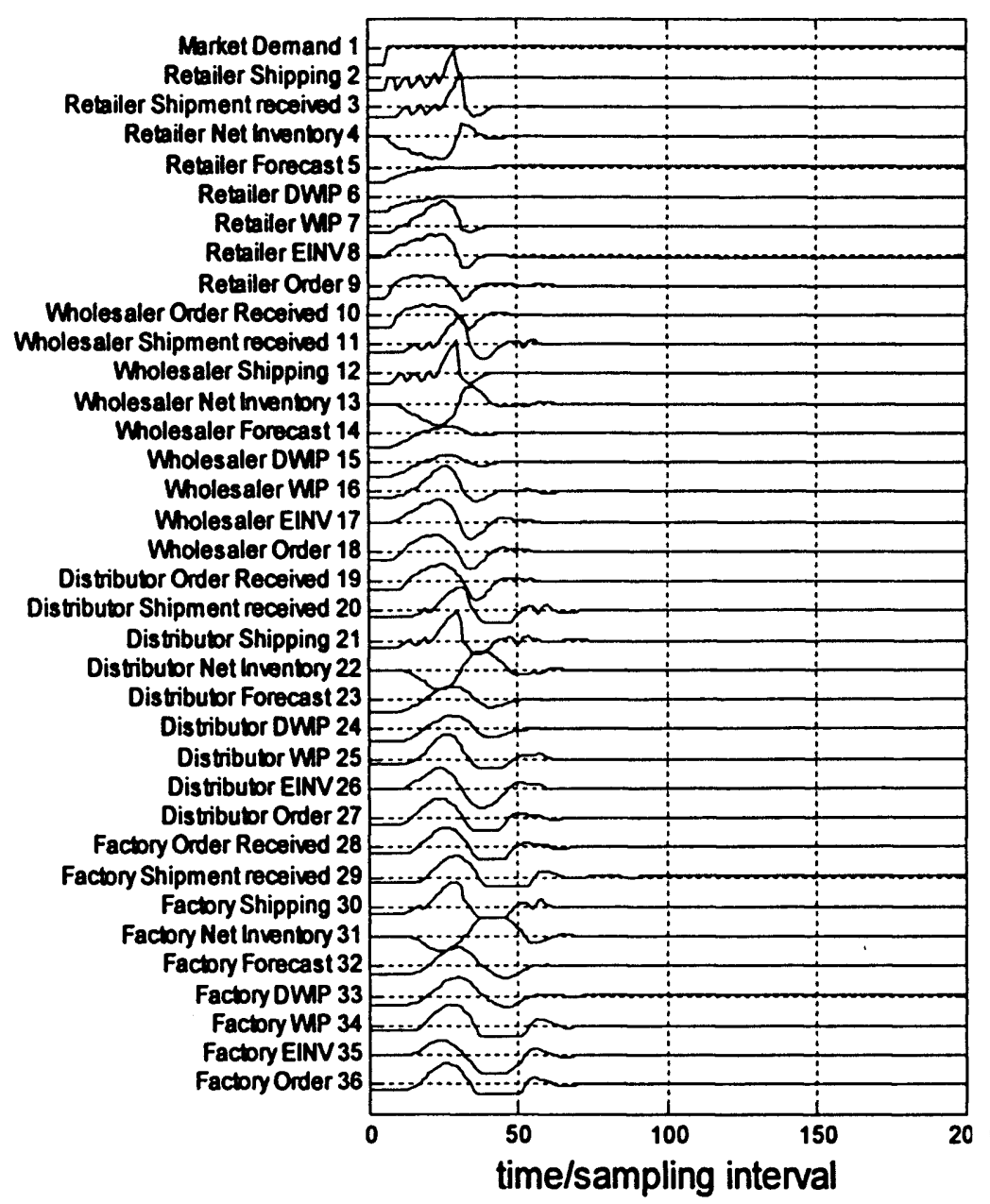

LT 223

Unoptimal parameters

No Batching

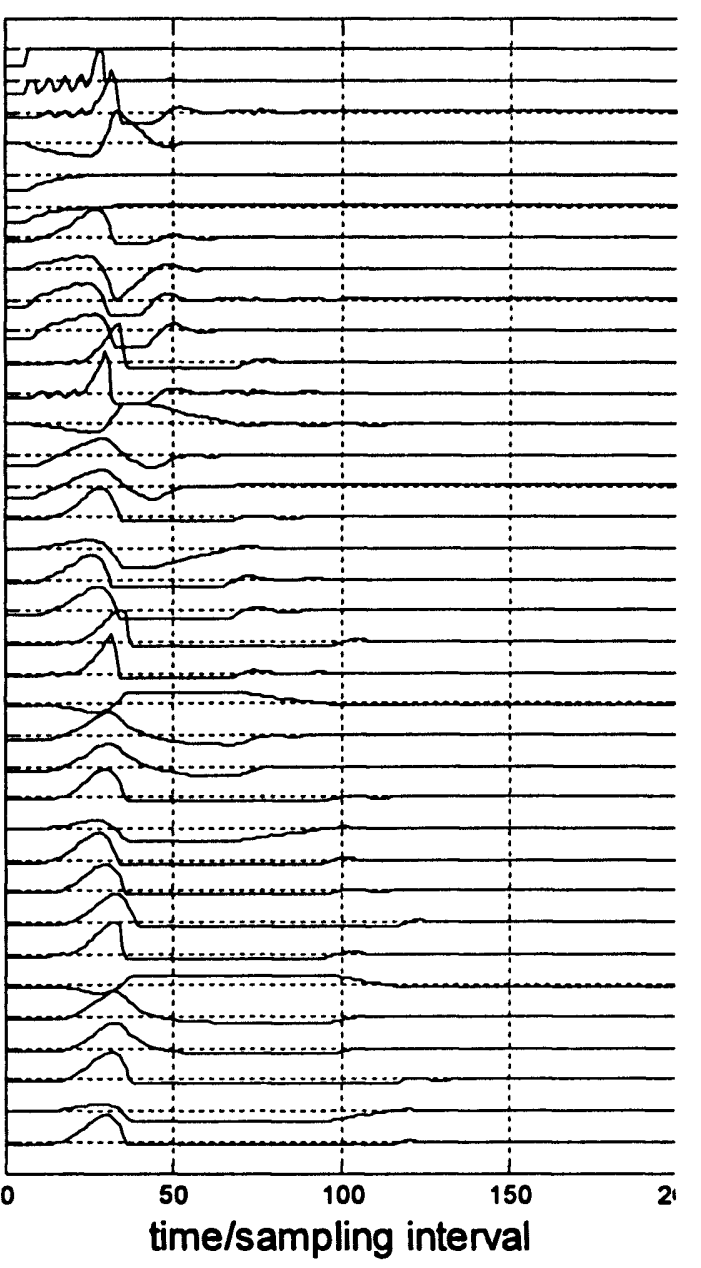

LT 334

Optimal parameters $100 \%$ Batching*

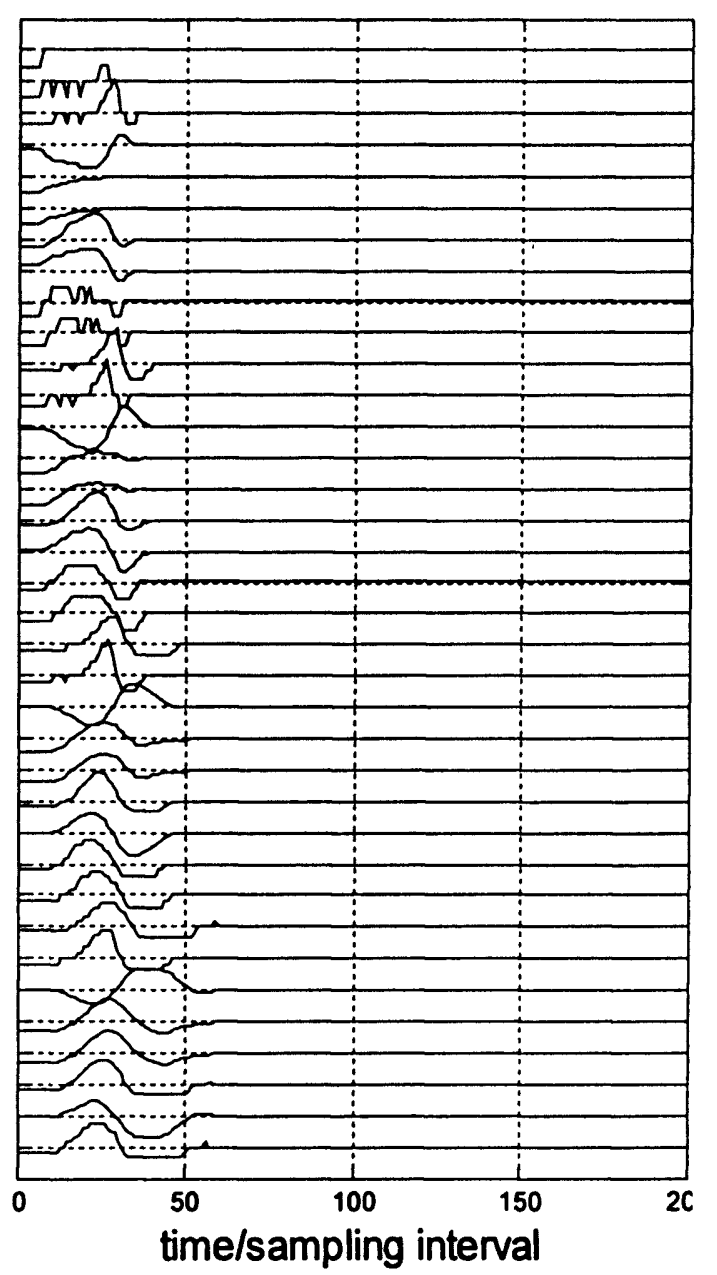

Figure 5.3 Time series profiles of variables from a simulated Beer game system under different settings subjected to a step change in demand (CONS)

LT a b c: a-ordering, b-shipping, c-production

* Orders are rounded up and shipping down to multiples of batch size; $100 \%$ batching with average demand per period of 4 means batch size of 4 
in this system.

A comparison of the variable profiles for the Unoptimal parameters option with that of Optimal parameters shows the greater amplitude of the former despite a significant masking of the difference from normalization. In a linear system, the intensity of rogue seasonality (as reflected in the amplitude of the relevant sinusoid) in the Unoptimal parameters case is greater than that in Optimal parameters, but here it is also seen to be true for the Beer game system which is non-linear. This was also evident in the FR analysis earlier. A comparison of the batching case with the non batching case shows the order and shipping variables (and therefore order received and shipment received variables also) being clipped because of batching (for example variables numbers $2,9,12$ and 18 in the far right panel of Figure 5.3). However, the other internal variables are not affected by the batching constraint and retain their smooth oscillatory profiles. Rogue seasonality characteristics are therefore modified but still retained in the Beer game system with batching. The batch size used in Figure 5.3 is same as the average demand i.e. orders are rounded up and shipments rounded down to a multiple of average demand (4 in this case) at each echelon.

\subsubsection{Rogue seasonality signature and index assessment}

Having analysed the generation of rogue seasonality in select variables through analytical FR analysis and then in most variables in a simulated Beer game system by time series analysis, the next issue is to test the effectiveness of the signature and index of rogue seasonality. A simulation output is used for this where the simulation was based on autoregressive of order two (AR 2: $\rho_{1}=0.1 \rho_{2}=-0.8$ ) as the demand process, ordering, shipping and production lead times of 2, 2, 3 respectively and Unoptimal parameters, Optimal parameters and Pass on orders (MTO) as the control systems. The last control system was used as a reference, similar to that in the linear systems case (Chapter 4). The rogue seasonality signature and index was computed using FT Total as the feature parameter combination which was identified as the best combination in Chapter 4. Figure 5.4 gives the signature and index for each of the three control systems based on FT Total. 
Pass on orders (MTO) system

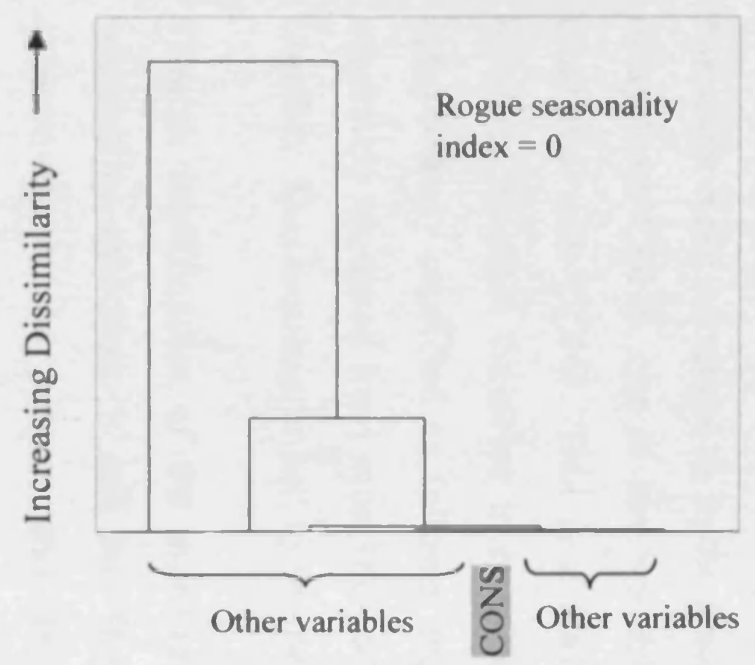

Hybrid MTO-MTS svstem

Optimal parameters (John et. al., 1994)

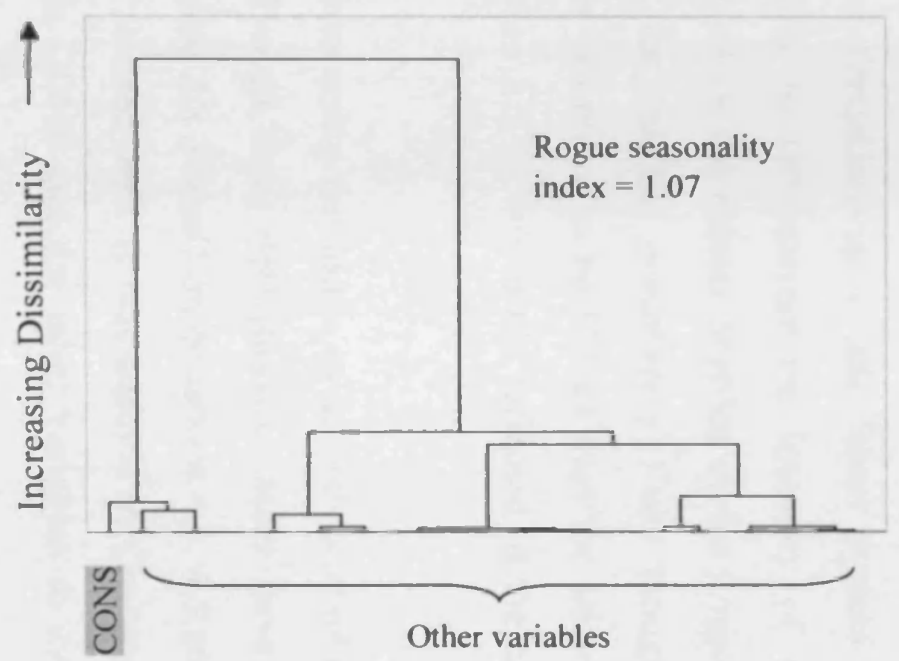

Hybrid MTO-MTS system

Unoptimal parameters (No pipeline feedback)

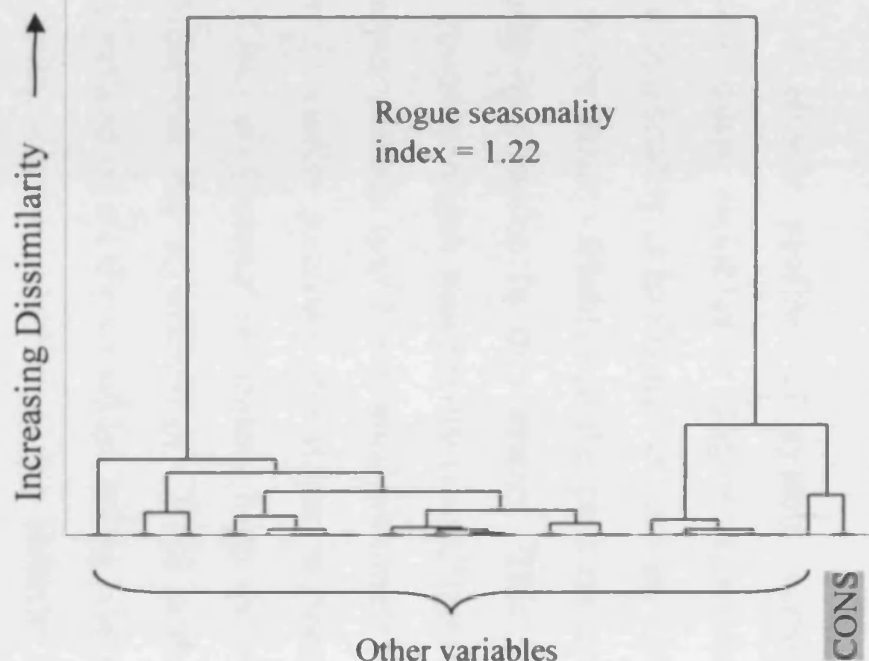

Figure 5.4. Impact of ordering practices on the signature and index of rogue seasonality based on FT (Total) feature parameter combination; Lead times considered: 2, 2, 3 (order, shipment and production); Exogenous demand used: AR (2): $\rho_{1}=0.1 \rho_{2}=-0.8$ 
The rogue seasonality signature is based on cluster profiles of system variables, specifically if CONS is clustered together with other variables or distinct from them. Where the latter is true, the presence of rogue seasonality is indicated, otherwise not. In Figure 5.4, CONS is seen to be clustered with the other variables in the pass on orders (MTO) system indicating no rogue seasonality generation in this system. This is an accurate assessment as MTO systems do not generate rogue seasonality (see Chapter 4). Similarly, in both the hybrid systems (Optimal parameters and Unoptimal parameters) in which rogue seasonality is generated as seen in earlier sections, the rogue seasonality signature again gives the correct indication; CONS is clustered separately from the other variables, with the latter clustered together. However, the separation of CONS with the variables is not complete as in the linear case; instead of all the variables being clustered away from CONS, one or two variables (shipping related variables at the Retailer) are clustered with CONS. This is a realistic possibility, especially in an analysis which involves multiple variables in multiple echelons. The signature definition needs to be appropriately modified as follows: rogue seasonality is considered present if CONS is separately clustered from most (not all) of the variables and these variables are clustered together. This requires 'most' to be specified as per the application context.

Though modification of the signature definition enables its continued use in rogue seasonality detection, it still has the same limitations as in the linear system case; subjectivity in the process and its inability to characterize the intensity of rogue seasonality. The latter limitation is evident when the cluster profiles of the Unoptimal parameters option is compared with that for Optimal parameters. Even though the intensity of rogue seasonality in the former is known to be higher than the latter (see Table 5.1 and the earlier discussion in Section 5.3.1), this is not reflected in the cluster profiles.

The alternative index based approach involves using the index as an indicator of rogue seasonality intensity in the supply chain. Though three definitions of index have been proposed earlier, specifically 'index', Alt 1 and Alt 2 based on equations 4.1, 4.3 and 4.4 respectively, 'index' based on equation 4.1 is used here. It was defined in Chapter 4 as the ratio of minimum dissimilarity between CONS and the other variables to average 
dissimilarity between all the other variables except CONS. The index values computed for the MTO, Hybrid system (Optimal parameters) and Hybrid system (Unoptimal parameters) are 0,1.07 and 1.22 respectively as seen in Figure 5.4. These accurately indicate the presence of rogue seasonality as well as discriminate systems having different rogue seasonality intensities (Optimal and Unoptimal parameters cases). The latter was not possible with the signature.

The index also does not require any modification to accommodate variables such as the shipping variable (which does not show rogue seasonality in contrast to most other variables and remains similar to CONS), as was required in case of the signature. This is because, though the presence of such variables reduces the numerator in the index, the remaining variables being aligned at common amplification frequencies reduces the denominator significantly to still yield a high index value which accurately characterises the presence and intensity of rogue seasonality. This is evident in the index values computed for Optimal and Unoptimal parameters options (1.07 and 1.22 respectively), which are relatively much higher than the 0 value associated with a system without rogue seasonality and therefore indicates both the presence as well as intensity of rogue seasonality accurately, despite one of the variables (shipping variable) being similar to CONS. Alternatively, this issue about one/a few inconsistent variables skewing the index value could be altogether avoided by using alternative indexes Alt 1 and Alt 2, which use average rather than minimum dissimilarity in the numerator.

The analysis in the preceding sections explained the generation of rogue seasonality in non linear systems with backlogs and batching, which use hybrid MTO-MTS ordering system and include shipment in the dynamics. The generation of rogue seasonality of greater intensity in the Unoptimal parameters case in comparison to Optimal parameter was also highlighted. Finally, the validity of the signature (with appropriate modification) and index for such systems was also established. The only issue with the previous analysis is that it was based on limited experimentation. Moreover, the index was computed with only one feature parameter combination (FT Total) and without considering alternative index definitions such as Alt 1 and Alt 2. A detailed simulation experiment incorporating all these aspects was therefore carried out. 


\subsection{Detailed simulation of the Beer game system}

The detailed simulation of the Beer game system had the following three parts as in the case of the linear system: generation of rogue seasonality of different characteristics and intensities in the system, inputting of the time series data of system variables so obtained into various transformations and using the respective features to derive the rogue seasonality index/indices (and this way assessing the appropriateness of the transformations/features) and finally repeating the above with alternative index definitions to assess their respective effectiveness. The simulation design is given in Table 5.3.

Table 5.3 - Simulation design for generating rogue seasonality of different characteristics and intensities in the Beer game system

\begin{tabular}{|c|c|}
\hline $\begin{array}{l}\text { Structure, number of echelons } \\
\text { and order of delay }\end{array}$ & $\begin{array}{l}\text { Beer game supply chain system } \\
\text { - Four echelon production-distribution structure } \\
\text { - Provision for backlogs between echelons } \\
\text { - Order infinity/pipeline delay for orders and shipments }\end{array}$ \\
\hline $\begin{array}{l}\text { Supply chain control } \\
\text { (Ordering) }\end{array}$ & $\begin{array}{l}\text { Hybrid MTO-MTS with Optimal parameters (John, 1994) } \\
\text { Hybrid MTO-MTS with Unoptimal parameters (no pipeline feedback) }\end{array}$ \\
\hline $\begin{array}{l}\text { Lead time (Ordering*- } \\
\text { Shipping*-Factory } \\
\text { production) }\end{array}$ & $\begin{array}{lll}1 & 1 & 2 \\
2 & 2 & 3 \\
3 & 3 & 4\end{array}$ \\
\hline $\begin{array}{l}\text { Demand process and } \\
\text { parameters }\end{array}$ & $\begin{array}{l}\operatorname{AR}(2): \rho_{1}=0.1 \rho_{2}=-0.8 \\
\operatorname{MA~(1):~} \theta=-0.2 \\
\operatorname{MA~(2):~} \theta_{1}=0.1 \theta_{2}=-0.8\end{array}$ \\
\hline $\begin{array}{l}\text { Batching** } \\
\text { (Orders and Shipping) }\end{array}$ & $\begin{array}{l}\text { No batching; } \\
\text { Batch size of } 50 \% \text { of average demand per period (Batch } 50 \% \text { ) } \\
\text { Batch size of } 100 \% \text { of average demand per period (Batch } 100 \% \text { ) }\end{array}$ \\
\hline $\begin{array}{l}\text { Variables input into } \\
\text { transformation } \\
\text { techniques/features for rogue } \\
\text { seasonality index computation }\end{array}$ & All variables, Only order and inventory \\
\hline ach based & $n$ numbers \\
\hline
\end{tabular}

* For all echelons

* Orders are rounded up and shipping down to multiples of batch size; Batch sizes used were 0 (No Batching), 2 (Batch 50\%) and 4 (Batch 100\%) with average demand per period considered equal to 4 
The simulated Beer game system structure is the same as used by Sterman (1989); four echelons, a sequential order and shipment flow with no information sharing between echelons, pipeline delays in ordering, shipping and production and infinite shipping capacity in the distribution echelons and production capacity at the factory. Different ordering heuristics (Optimal and Unoptimal parameters) are applied to generate rogue seasonalities of different intensities. The impact of these heuristics on rogue seasonality generation has been discussed earlier.

With regard to choice of demand processes and parameters, these have been considered as their low frequency (amplification range) frequency content is different (see Appendix C) which means that they would generate rogue seasonality of different intensities. The logic for this is the same as in the linear case (Chapter 4, Section 4.3.1), though here there are additional complexities from backlogs, batching and shipment dynamics.

With regard to choice of lead times (or delays), the base case used is 2, 2, 3 (ordering, shipping, production delay at the factory) which is the same as that used by Sterman (1989). Alternative delays of $1,1,2$ and 3, 3, 4 are also considered to assess the sensitivity of the findings to delays. Though the intensity of oscillation is unaffected by a change in lead time in linear systems and only the oscillation frequency changes ( $\max F R$ is independent of lead time $T_{p}$ for many variables as seen in Table 5.1), this may not apply in a system with backlogs and associated non-linearities. The choice of lead times also helps in assessing the robustness of the index. Unlike the linear system analysis, where the same delay was applied to all the three echelons (for any choice of delay), here the lead time (production) for the factory echelon is different from the lead times (ordering + shipping) in other echelons. For example, lead times of 2, 2, 3 mean that while each of the Retailer, Wholesaler and Distributor echelons have a delay of 4 , the factory has a delay of 3 (production). A similar logic applies in the case of lead times 3, 3, 4. Since, the relevant amplification frequencies, $\omega_{\max }$ FR and $\omega_{\text {crossover }}$ are both related to delay, the difference in delays between the distribution and the factory echelons misaligns their cyclic profiles, which helps in assessing whether the index was a robust indicator under such circumstances. 
Next, the impact of alternative batch sizes on the generation and presentation of rogue seasonality is assessed by considering the following cases: no batching and batch sizes of $50 \%$ and $100 \%$ of average demand per period (translates to batch sizes of 0,2 and 4 for the average demand period used of 4). For any choice of batch size, it is the same in all echelons. The batch sizes considered are appropriate as these are essentially batching in transportation associated with the distribution structure used.

Finally, in terms of the system variables used for index computation, besides all variables, order and inventory variables are also used and assessed. Thirty independent replications were generated for each simulated case based on common random numbers. Excel $\odot$ together with Matlab $\odot$ was used for the data generation process.

In terms of the transformation techniques, the same techniques, features and their parameters as are used as in the linear system analysis (See Table 4.3). This is done to ensure that the findings from the two analyses could be compared. The alternative index definitions evaluated are also the same as in equations 4.1, 4.3 and 4.4.

\subsection{Analysis of output from detailed simulation of the Beer game system}

The two cases, all variables used and only order and inventory variables used in index computation are discussed separately below.

\subsubsection{Case IV: All variables used in index computation}

The analysis is presented in three sub-sections. First, the impact of different simulation variants on rogue seasonality is assessed using FR of select system variables computed from the simulated Beer game. Next, the index values for simulated systems (demand processes/lead times/ordering policies/batch sizes) based on different feature parameter combinations are analysed. This includes comparing the values across demand processes, ordering policies, lead times and batch sizes as well as with corresponding linear systems to assess whether the index was behaving as expected. Structured assessment, based on the criteria of consistency, discrimination ability and stochastic robustness, of alternative feature parameter combinations is also covered in this sub 
section. In the final sub-section, correlation between the three alternative index definitions proposed in Chapter 4 is discussed.

\subsubsection{Understanding the impact of different factors on rogue seasonality}

In a system with backlogs such as the Beer game, the impact of a combination of factors such as ordering policies, demand processes and parameters and lead time combinations on rogue seasonality is essentially the impact of each factor in a linear system and the alterations to the same from backlogs and batching (non-linearities). Knowledge of the extent of backlogs created (or alternatively the fill rate achieved) from different combinations of factors used in the simulation is therefore necessary. The average fill rate (a measure of backlogs) from 30 replications for the distributor and factory echelons (where maximum backlogs are created) for different simulation combinations was between $80 \%$ and $100 \%$. This is a realistic fill rate for a hybrid system; pure MTO systems have higher backlogs/lower fill rates and pure MTS systems much lower. Next we understand the impact of each simulation variant on rogue seasonality generation. FR profiles averaged over 30 replications are used for this. It is to be noted that backlogs and their impact are an intrinsic part of each analysis.

a) Impact of lead time: To understand the impact of lead times (or delays) on rogue seasonality, the Beer game is simulated with lead times of $(1,1,2),(2,2,3)$ and $(3,3,4)$ with MA (1), $\theta=-0.2$ used as the exogenous demand and ordering based on Unoptimal parameters. The echelon-wise FR profiles computed from the simulation data for ORATE/CONS, COMRATE/CONS, AINV/CONS and WIP/CONS are given in Appendix H.1. The FR profiles show that for a particular lead time case, all variables in all echelons have the same frequency (slightly different for the factory as discussed earlier) associated with the peak FR value; and this is true for each of the analysed lead time cases. The characteristic presentation of rogue seasonality, as discussed in this chapter earlier as well as in Chapter 4, is therefore evident. In terms of the impact of lead time on rogue seasonality intensity, in a linear system there is no impact as seen in Table 5.1. However, this is also apparent in this system with backlogs; the peak FR values for variables such as ORATE/CONS are approximately equivalent for alternative lead time 
cases in the case of each echelon. The peak FR values for variables such as AINV/CONS and WIP/CONS are, however, different for different lead time cases as expected because they are proportional to lead time.

b) Impact of batching: The impact of batching is analysed similarly as lead time and depicted in Appendix H.2 with batch sizes of 50\% and 100\% being used (defined with reference to average demand per period which was considered to be 4 ; means batch sizes of 2 and 4 are used). The FR profiles show a significant irregularity (lack of smoothness) which is on account of batching as well as backlog related non-linearities. However, the characteristic presentation of rogue seasonality is still evident from the profiles of the variables which means that the signature and index of rogue seasonality are valid for cases with batching as well. Similar to the lead time case, the peak FR values for variables such as ORATE/CONS do not change significantly with batch sizes. This indicates the rogue seasonality intensities for different batch sizes to be not very different from each other.

The impact of ordering policies (Optimal, Unoptimal parameters) in a system with backlogs has been discussed earlier in Section 5.4.1. Finally, regarding the impact of alternative demand processes and parameters in a system with backlogs, the basic logic used in the linear system analysis (those with higher spectral energy in the low frequency amplification range frequencies would show greater rogue seasonality intensity) is also applicable here. However, in a system with backlogs, the regularity of the profiles would be lower as seen in the batching and lead time analysis earlier.

\subsubsection{Analysis of index values based on alternative feature parameter combinations}

The average index values (30 replications), based on different feature parameter combinations for all demand process, lead time, ordering process (Optimal/Unoptimal) and batch size combinations are given in Appendix I.1. We first assess whether the index is able to capture the expected rogue seasonality characteristics and intensities discussed in the previous sub-section. The index based on FT Total, which was identified as the best feature parameter combination in the linear systems analysis, is used for this. 
a) The system variables in a hybrid MTO-MTS system with Optimal parameters (delay order infinity assumed) have a $\max F R$ value of 1.69 while it is 2.31 for those in MTS delay order infinity system. The intensity of rogue seasonality in the former is lower than in the latter and this should be reflected in their relative index values. FT Total index values for the hybrid MTO-MTS system with Optimal parameters (Appendix I.1) are actually lower than that for MTS delay order infinity system (Appendix E) in each corresponding case.

b) In terms of impact of change in lead time on rogue seasonality intensity, in a linear system there should be no impact but in system with backlogs, as used here, there could be a marginal impact from the latter. Here again the FT Total accurately reflects this. For example, the index based on FT Total for systems with lead times 334 and 223 are 1.22 and 1.21 (demand process AR (2) $\rho_{1} 0.1 \rho_{2}-0.8$, Unoptimal parameters; no batching). The behaviour of the index again is as expected.

c) Similar to lead time, batching (specifically the batch sizes used) is also expected to cause a minimum change in the rogue seasonality intensity as discussed earlier using the FR analysis. The index based on FT Total accurately reflects this. For example, the index values for no batch, batch (50\% or 2$)$, batch (100\% or 4$)$ are $1.22,1.19,1.19$ (demand process AR (2) $\rho_{1} 0.1 \rho_{2}-0.8$, LT 334, Unoptimal parameters)

The index, if based on the appropriate feature parameter combination, is therefore able to characterise the rogue seasonality intensity even in systems with non-linearities from backlogs and batching. While we previously used the index based on FT Total (it being the best in the linear system), there is a need to assess if it is the best for the current context as well. Alternative feature parameter combinations are therefore evaluated in a structured way using the criteria of consistency, discrimination ability and stochastic robustness (see Section 4.4.1.2). While the latter two criteria are used exactly in the same way as in Chapter 4, for the consistency criteria, consistency in relation to ordering policy (Optimal and Unoptimal parameters) rather than order of delay is assessed. The structured analysis is given in Tables $5.4 \mathrm{a}, \mathrm{b}$ and $\mathrm{c}$. 
Table 5.4: Structured evaluation of different feature parameter combinations for rogue seasonality index computation in the Beer game system;

\section{All variables used in computation of the index}

\section{Table 5.4a}

Consistency of rogue seasonality index for different demand and ordering processes

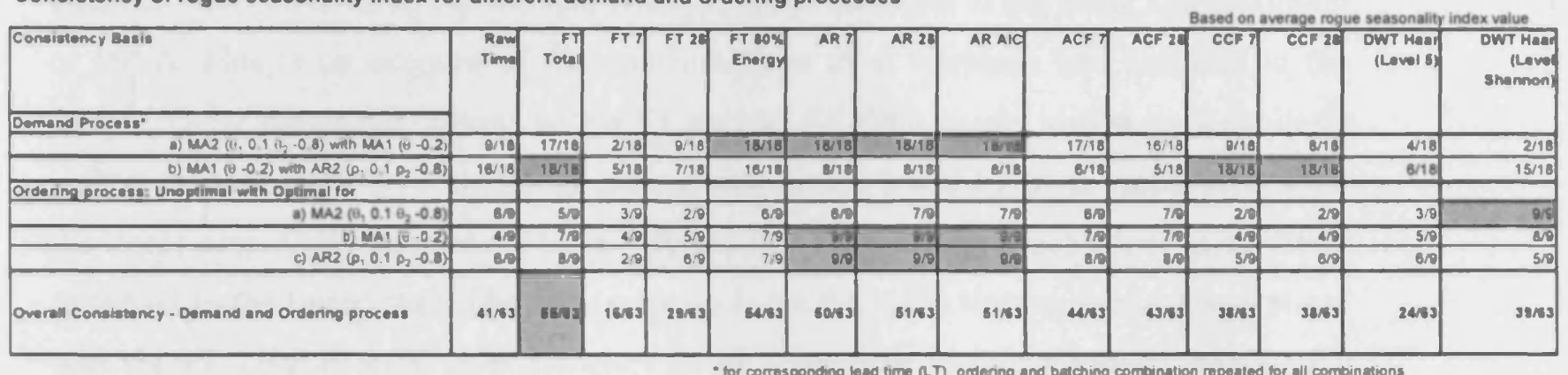

- for corresponding lead time (LT), ordering and bacching combination repeated for all combinations

\section{Table $5.4 \mathrm{~b}$}

Rank analysis based on the magnitude of rogue seasonality index

(For assessing the discrimination ability of the index derived from different feature parameter combinations)

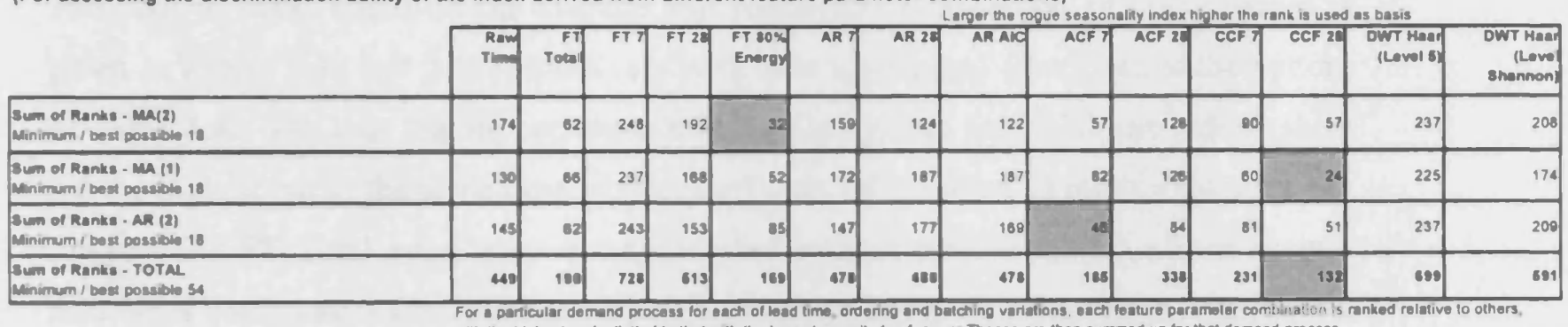

\section{Table $5.4 \mathrm{c}$}

Rank analysis based on the coefficient of variation of rogue seasonality index

(For assessing the stochastic stability of the index derived from different feature parameter combinations)

\begin{tabular}{|c|c|c|c|c|c|c|c|c|c|c|c|c|c|c|}
\hline & $\begin{array}{l}\text { Ram } \\
\text { Thing }\end{array}$ & $\begin{array}{l}\text { FT } \\
\text { Toen }\end{array}$ & FT? & FT2E & $\begin{array}{l}\text { FT Boy } \\
\text { Energy }\end{array}$ & ART & AR 2E & ARAIC & ACF & ACF 8 & CCF 7 & CCF 2 & $\begin{array}{l}\text { DWT Hear } \\
\text { (Lowi s) }\end{array}$ & $\begin{array}{l}\text { DWT Hal } \\
\text { (Level } \\
\text { shannon, }\end{array}$ \\
\hline 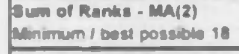 & 1여 & 37 & 252 & 197 & 93 & 148 & 147 & 148 & 150 & 160 & 84 & 70 & 208 & 178 \\
\hline 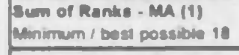 & 18 & 43 & 244 & 150 & 81 & 180 & 175 & 187 & 102 & 152 & 80 & 71 & 175 & 183 \\
\hline 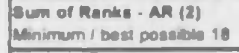 & 18 & 73 & 252 & 172 & 104 & 117 & 153 & 162 & 140 & 154 & 60 & 70 & 128 & 117 \\
\hline $\begin{array}{l}\text { Sum of Ranike - TOTAL } \\
\text { Minimum I dest possibie } 34\end{array}$ & 14) & 183 & 748 & 828 & 360 & 408 & 676 & 4ne & 470 & 488 & $1 \mathrm{M}$ & 228 & 572 & A7e \\
\hline
\end{tabular}

\begin{tabular}{|c|c|c|c|c|c|c|c|c|c|c|c|c|c|c|}
\hline & $\begin{array}{l}\text { Ren } \\
\text { Time }\end{array}$ & $\begin{array}{r}F T \\
\text { Total }\end{array}$ & $\mathrm{FTT}$ & FT 28 & $\begin{array}{l}\text { FT 80x } \\
\text { Energy }\end{array}$ & $A R T$ & AR 2C & AR AIC & ACF ज्ञ & ACF 2I & C्CF & CCF 2 이 & $\begin{array}{l}\text { DWT Hant } \\
\text { (Level of }\end{array}$ & $\begin{array}{l}\text { DWT Kme? } \\
\text { (Love) } \\
\text { shannon) }\end{array}$ \\
\hline 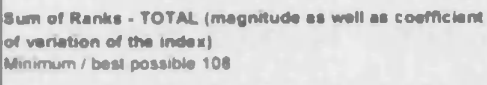 & 803 & 345: & 1476 & 1041 & 657| & 923 & 963 & 176 & (5) & Iad & 418 & 341 & 1279 & 1070 \\
\hline
\end{tabular}


Table 5.4a gives the consistency of the index with respect to demand process (and parameters) and ordering processes (Unoptimal and Optimal parameters) for different feature parameter combinations. FT Total shows the highest consistency amongst all feature parameter combinations as in the linear case. It has a high consistency in absolute terms as well (55/63 or 87\%), though, which is lower than that in the linear system (68/69 or $99 \%$ ). This is on account of the non-linearities from backlogs and batching in the former. Other parameter options within FT such as FT $80 \%$ energy also show a similarly high consistency as FT Total, though consistency for FT 7 and FT 28 is much lower as in the linear case. Consistencies for time, AR and DWT features are also lower as similarly observed in the linear case. The only surprise is for the ACF feature; while it was at par with the FT Total in terms of consistency in the linear case, it is much lower than the FT Total in this case and only marginally better than the time domain.

The sum of ranks based on the average and coefficient of variation of index values is given in Tables 5.4b and 5.4c respectively with their aggregated sum given at the bottom of Table 5.4c. The best feature parameter combination is that for which the index values are the highest but at the same time whose coefficient of variation of index values are the lowest. The FT Total has the lowest aggregated sum of ranks amongst all the feature parameter combinations considered, indicating it to be the most balanced in meeting the above considerations which was also the case in the linear system analysis (see Table 4.5). As regards other feature parameter combinations, they are similarly placed relative to each other in terms of aggregate ranks as in the linear case.

Based on all the three criteria, the FT Total feature parameter combination was found to be the best for rogue seasonality index computation with FT $80 \%$ Energy a close second as in the linear case. Other findings are also similar to that in the linear case. The index computed in the time domain was found to be a less valid measure of rogue seasonality (low consistency) and a less effective discriminator of rogue seasonalities of different intensities (low index values). AR and DWT features were found to be among the worst on each criterion while CCF features showed low consistency. The only surprising finding was with regard to ACF features which showed lower consistency both in 
absolute terms as well as relative to FT features (FT Total and FT $80 \%$ Energy) compared to the linear system.

\subsubsection{Comparison of alternative index definitions}

The correlation between the alternative index definitions (based on equations 4.1, 4.3 and 4.3) for different features are given in Table 5.5. These were computed in a similar fashion as in the linear system analysis. Table 5.5 highlights the fact that the correlation between alternative index definitions for most of the features (FT, AR Model, ACF, CCF) is high. This means that when these features are used, any of the three index definitions could be used for index computation (and therefore rogue seasonality detection) without loss of accuracy. However, the comparison of the correlation coefficients given in Table 5.5 with those for the linear system given in Table 4.6, show the former to be lower which again can be explained by the greater system complexity (and its associated impact on the data variables) from the simultaneous application of backlogs, batching and other factors. The correlation between index definitions in the time domain and using DWT coefficients is low as was seen in the linear case.

Table 5.5: Correlation between alternative rogue seasonality indices in the Beer game system - $\underline{\text { All }}$ variables used in computation of the indices

\begin{tabular}{|l|c|c|c|}
\hline & Index and Alt & Index and Alt & Alt 1 and Alt 2 \\
\hline Time & 0.67 & 0.84 & 0.37 \\
FT & 0.84 & 0.97 & 0.75 \\
AR Model & 0.85 & 0.98 & 0.84 \\
ACF & 0.87 & 0.99 & 0.86 \\
CCF & 0.79 & 0.97 & 0.66 \\
DWT & 0.64 & 0.64 & 0.40 \\
\hline \multicolumn{3}{|c|}{ Parameter options for each feature have been combined together } \\
\hline
\end{tabular}

\subsubsection{Case V: Only order and inventory variables used in the index computation}

The average and the coefficient of variation of the index values are given in Appendices J.1 and J.2 respectively while Appendix J.3 gives the structured evaluation of alternative feature parameter combinations based on the criteria of consistency, discrimination 
ability and stochastic robustness. Finally, Appendix J.4 gives the correlation between alternative index definitions for different features. A comparison of the average index values between an "all variables used" case (Appendix I.1) and the "orders and inventory variables used" case (Appendix J.1) for feature parameter combinations such as FT Total shows only small differences. For example, in the "all variables used" case, the average index values for Unoptimal and Optimal parameters ordering are 1.22 and 1.18 (lead times 334 , no batching, excited with the demand process AR (2): $\rho_{1} 0.1, \rho_{2}-0.8$ ). The corresponding values for the "order and inventory variables used" case are 1.45 and 1.30. The same is true for the other demand processes as well. This implies that use of fewer but representative variables such as order and inventory variables, give a similar indication of rogue seasonality intensity.

The structured evaluation of alternative feature parameter combinations, which is given in Appendix J.3, shows the FT Total to be among the most consistent feature parameter combination. The index value based on the FT Total also provides the maximum discrimination between rogue seasonalities of different intensities while being stable in stochastic terms. FT $80 \%$ Energy is close to the FT Total on all of the evaluation criteria. ACF features on the other hand, show similar consistency and discrimination ability as the FT Total, but were found to be lacking in the stochastic robustness criteria. Other features such as AR, DWT, Time and CCF also were found to be lacking in one or more criteria.

Finally, J.4 gives the correlation coefficient between the alternative index definitions where again the results are similar to the "all variables used" case (Table 5.5) i.e. high correlation coefficient for the FT, AR model, ACF and CCF features and low correlation coefficient for the time domain and DWT coefficients.

\subsection{Conclusions}

This chapter analyzed rogue seasonality generation in a non-linear (Beer game) system. Backlogs, batching in ordering and shipping, shipping variable and hybrid MTO-MTS system with Optimal and Unoptimal parameters were applied to the system and their impacts analysed. The outputs from the simulated system were used to assess the 
effectiveness of the rogue seasonality signature and index, together with the best features (and parameters) to be used for deriving them.

The signature of rogue seasonality was found to be valid for the Beer game system notwithstanding its greater complexity than the linear system used in Chapter 4 with four echelons, a greater number of variables, echelons having different lead times and all the other factors including non-linearities mentioned in the previous paragraph. The signature was able to accurately record the rogue seasonality generation. However, unlike the signature in the linear system, where all the other variables were clustered together and separate from CONS, in this case one variable (the shipping variable) continued to be clustered with CONS. Recognizing that this could arise in real systems as well, rogue seasonality definition was modified as follows: rogue seasonality is considered present if CONS is separately clustered from most of the variables (the threshold needs to be defined) and these variables are clustered together.

The rogue seasonality index was found to be an accurate barometer of rogue seasonality intensity. While the index value for the make to order (MTO) system was 0 , it was higher $(>1)$ for the hybrid MTO-MTS system with Optimal parameters and the highest for the hybrid system with Unoptimal parameters. These index values are as expected based on their respective rogue seasonality intensities (from analytical frequency response (FR) analysis).

In terms of feature parameter combinations to be used for index computation, FT Total was found to be the best followed closely by FT $80 \%$ Energy. Index values derived from them showed high consistency (both in absolute and relative terms), discrimination ability and stochastic stability. This finding is similar to that for the linear system. Relative performance of the other feature parameter combinations on these criteria is also the same as their performance in the linear system. In terms of the appropriateness of alternative index definitions, the correlation between the index values based on these definitions was high for FT, AR Model, ACF and CCF features as in the linear case. However, the extent of correlation (reflected in the value of correlation coefficient) in the Beer game system was lower. This is on account of the greater system complexity (and 
its associated impact on the data variables) from the simultaneous application of backlogs, batching and other factors. Finally, use of order and inventory variables gave the same overall findings as when all the variables are used (as discussed above). 


\section{Chapter 6 - Analysis of rogue seasonality in empirical}

\section{contexts}

Chapters 4 and 5 used simulated linear and non-linear supply chain contexts to analyze rogue seasonality. Rogue seasonality's characteristic presentation as multiple system variables oscillating in sync with each other, with the oscillation not present in the exogenous demand, was highlighted. The signature based on the clustering profiles of variables in relation to exogenous demand was found to be an effective indicator of the presence/absence of rogue seasonality. However, to get a sense of the intensity rather than just the presence or absence of rogue seasonality, a numerical index was proposed and tested and found to be a valid measure. It is based on the dissimilarity between the other variables and exogenous demand in relation to the dissimilarity between the other variables themselves. Different time series transformation techniques were explored for computing the index out of which the FT Total feature parameter combination was found to be the best followed closely by FT $80 \%$ Energy. In this chapter, time series data from two empirical contexts are used to validate the above findings on rogue seasonality.

First a steel case study is analysed. Steel being an industrial product is characterized by relatively stable demand profiles and (consequently) similarly stable profiles of other supply chain variables. Monthly sampled data could therefore accurately capture the dynamics associated with rogue seasonality in this case and hence used. The second context is of a supply chain network in the grocery sector (a more volatile sector) and where, time series data of supply chain variables sampled at a higher rate (than monthly) is used.

Each case study is discussed separately one after the other as their rogue seasonality contexts are different. First, the steel case study context is explained which is followed by a discussion of the methods used by Thornhill and Naim (2006) and their findings. The case study and data used here is the same as used by them and this would bring more clarity to the contribution of the present work. Next, the different kinds of analysis performed on the data is discussed which are: 
- Transformation of the data to different domains, and use of the features from these domains for clustering based signature formulation

- Index computation from alternative feature parameter combinations to identify the best one for the same and therefore for rogue seasonality detection

- Assessing the impact of using order and inventory variables instead of all available variables on the index values

- Comparing the index values derived from alternative index definitions considered as in equations $4.1,4.3$ and 4.4

After the analysis of the steel case study, the grocery case study is similarly analysed, discussed and presented.

6.1 Description of the steel case study context and its analysis based on the spectral principal component technique

The steel supply network used in this study is depicted in Figure 6.1 which shows the flow of order and material across the network. The network consists of four autonomous business units: Steel works, Section mill, Bar mill and Rod mill. The Steel works produces steel billets of different qualities and specifications which are used as raw materials by the other three mills. These three mills then produce an even wider range of products which are sold to customers which include stock holders as well as end users. Though being a part of the same holding group, the four units are managed independently and operate as separate profit centres. Their production control is also not centralised with scheduling done independently of the others at each unit.

The study being information centric, data on different system variables across the network was required, which was originally collected from the company's management information systems. However, for the purposes of the present study, this data was already available, which includes variables such as customer orders, production output, dispatches, receipts, and inventory levels. These variables are similar to those used in the simulation analysis. Figure 6.1 lists these variables for each business unit. The meanings of these variables are mostly self-evident from their descriptions; the order book variables (numbers 29,30 ) are the orders accepted by a company that have not yet been delivered 


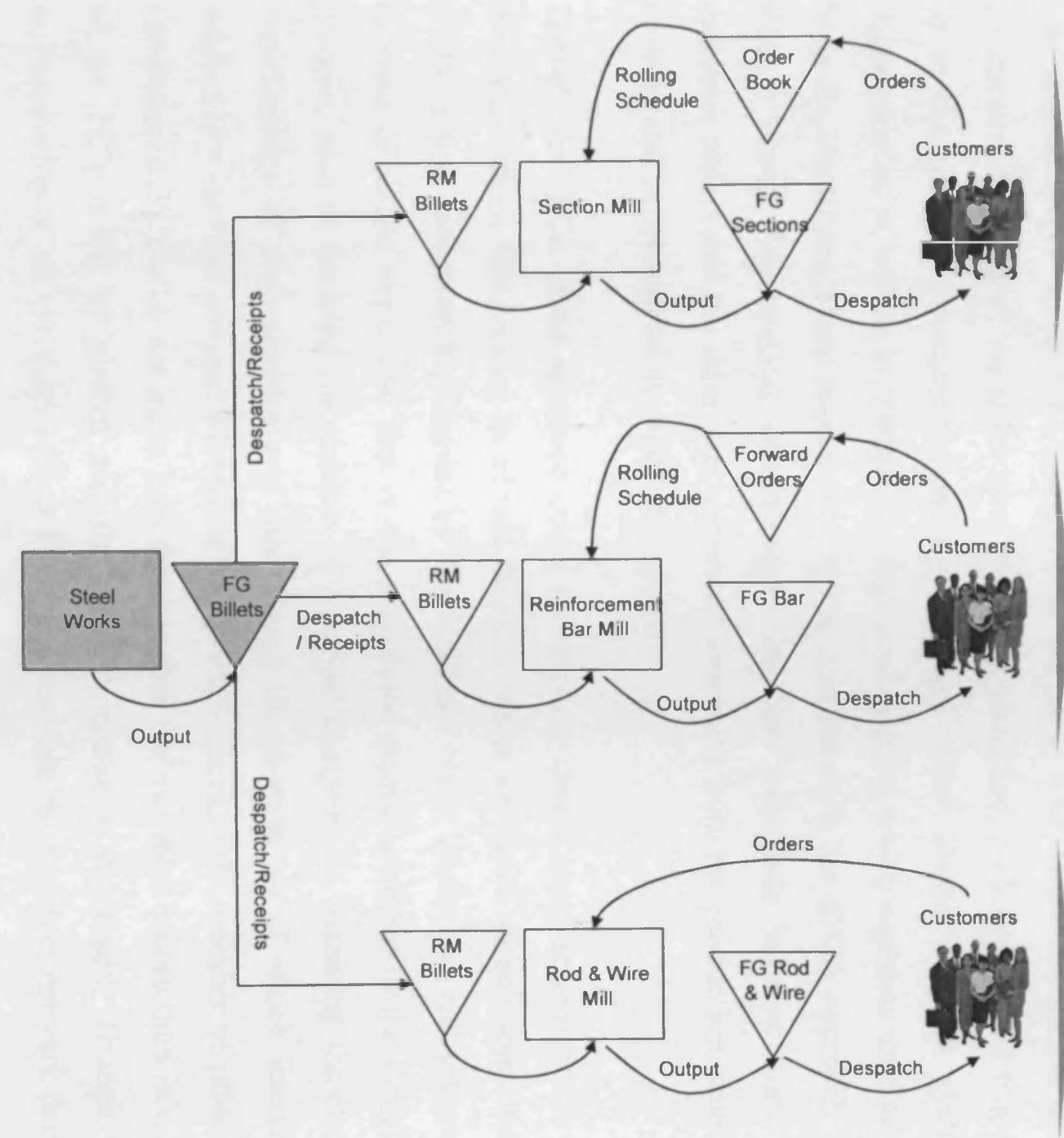

MTS: Steel Works-Section Mill Dyad

$\begin{array}{ll}\text { Steol Works variables } & \text { Sirction Mill variables } \\ \text { FG Billets Total Stocks ( } 38 \text { ) } & \text { Section Mill Total Orders ( } 9 \text { ) } \\ \text { FG Billets Despatches to Bar/Section mill ( 40) } & \text { Section Mill Production ( } 1 \text { ) } \\ \text { FG Billets Production ( 43 ) } & \text { Section Mill Despatches Total ( } 6 \text { ) } \\ & \text { Section Mill Receipts from FG ( 10) } \\ & \text { Section Mill Billet Stocks total ( } 13 \text { ) } \\ & \text { Section Mill Total Stocks ( 14 ) } \\ & \text { Order Book Section Mill ( } 30 \text { ) }\end{array}$

MTS: Steel Works-Bar Mill Dyad

Steel Works variables

FG Billets Total Stocks ( 38 )

to Bar/Section mill ( 40 )

Bar Mill Total Orders ( 23

Bar Mill Production ( 15 )

Bar Mill Despatches Total ( 20 )

Bar Mill Receipts from FG ( 24

Bar Mill Billet Stocks total ( 27 )

Bar Mill Total Stocks ( 28 )

Bar Mill Order Book ( 29)

\section{MTO: Steel Works-Rod Mill Dyad}

Steel Warks variables

FG Billets Total Stocks ( 38 )

FG Billets Despatches to Rod mill ( 39 )

Rod Mill variables

Rod Mill Total Orders ( 37 )

Rod Mill Production ( 32 )

Rod Mill Billet Stocks total ( 35 )

Rod Mill Total Stocks ( 36 )

Each variable is numbered to facilitate ease in representation

Figure 6.1. Steel case study network, its constituent dyads and the variables used in analyzing them 
to the customer and are akin to backlogs. Monthly time series data for each variable was available for 6 years ( 72 months).

The steel case study context used in this chapter has been used by Thornhill and Naim (2006) to demonstrate the effectiveness of the spectral principal component analysis (SPCA) technique in identifying rogue seasonality. SPCA is derived from PCA where PCA (principal components analysis) is the dimensional reduction technique which converts a number of correlated features of the data into a smaller number of uncorrelated features called principal components (PCs), with the first few PCs being used to represent the original data for further analysis. In SPCA, PCA is done for the spectra so that time delays or phase shifts caused by network dynamics are eliminated from the analysis. In Thornhill and Naim's study, SPCA was used for identifying rogue seasonality. The time series data for all the variables in the network was first normalized and detrended after which it was transformed into a spectra representation (using Fourier transform). Then, the dimensionality of the spectra for each variable was reduced to 3 dimensions using PCA, so as to plot each variable in the 3 dimensions. Clusters of variables were manually identified, with the clusters mapped on to the network structure to identify variables which shared common cyclical characteristics as well as in relation to exogenous demand which was then used as the basis for identifying rogue seasonality. In the steel network, the SPCA approach was able to detect two cyclical disturbances. One was externally induced from the customer orders and the other was generated internally from the production planning systems and hence termed as rogue seasonality.

Though the SPCA based approach could successfully detect rogue seasonality in the steel network, it was lacking in certain respects which are practical and scientific in nature. These have been highlighted by Thornhill and Naim themselves and suggested as areas of future work. The first is the significant manual element in the detection process, such as deciding the number of principal components, assessing the cluster memberships of the variables and interpreting the clusters all of which introduce subjectivity into the process. Moreover, the process requires the number of principal components (PC) to be not more than three so that the variables represented in terms of the PC's could be plotted and the clusters manually visualized. Though this representation of all variables with 3 PC's was possible for the steel network data, it 
may not work in other contexts having different data characteristics. The second issue is with regard to the use of just one technique, SPCA, in the study which was not compared to other potential techniques to establish its superiority or otherwise.

The aim here is therefore, to use the steel case study data to validate the signature and index of rogue seasonality so that the case for automatic rogue seasonality detection based on these could be strengthened. It is also to establish the best time series transformation technique (and feature) for signature formulation and index computation using real data.

\subsection{Rogue seasonality analysis of the steel case study based on signature and index}

The steel case study network is not analysed as such but is instead broken up into its three constituent dyads as seen in Figure 6.1: Steel works-Section mill, Steel worksBar mill and Steel works-Rod mill and analysed separately. This is because Thornhill and Naim's study determined the mills to be using different ordering strategies. The Section and Bar mills used the make to stock (MTS) and the Rod mill the make to order (MTO) strategy with the former showing rogue seasonality characteristics and the latter not showing it. By splitting the network into dyads, systems with contrasting rogue seasonality characteristics could be analysed. However, it is important to note that even though these dyadic systems are being termed as MTO and MTS systems, actually they are all hybrid MTO-MTS systems but with orders having a greater influence in the ordering decision in the former (MTO) and lesser in the latter (MTS). This is because for industrial products of the kind considered here, specifically steel products, hybrid strategies are generally used. Pure MTO policies are not used as economies of scale in production means build up of stocks. Likewise, demand from customers is specific and hence the pure MTS policy is also not used. In the case of the steel case study the hybrid nature of the systems is borne out by the variables used (see Figure 6.1). For example, the Section and Bar mills have order book variables implying that these are not operating on a pure MTS basis. Similarly, the Rod mill has a stocks variable which implies that it is also not operating on a pure MTO basis.

Each dyad is analysed separately on the basis of its system variables which includes the signature as well as the index values derived from different feature parameter 
combinations. The sequence of steps used in the analyses are as follows:

- The time series data of different variables was checked for consistency using analytical techniques.

- Data was de-trended so as to avoid its appearance in the zero frequency channel. Also, all low frequencies reflecting long term patterns (below a specified threshold) were filtered. The threshold used here is the same as that used by Thornhill and Naim (2006) at frequency 0.125 (time period greater than 8 months).

- For the index computation, all common frequencies shared between orders and other variables reflecting transmission of exogenous demand were filtered out in line with the discussion in Section 4.4.2. For the Steel works-Section mill dyad, this corresponded to the frequencies 0.25 (4 month cycle) and 0.17 (6 month cycle), for the Steel works-Bar mill it was 0.25 (4 month cycle) and for the Steel works-Rod mill 0.25 ( 4 month cycle) and 0.33 ( 3 month cycle). Filtering is done by replacing the values in the frequency channels (to be filtered) with zero.

- Data was normalised and each of FT, ACF and CCF transformations were applied to the data, with time domain used as the reference. AR and DWT transformations were ignored as they were found to be ineffective for rogue seasonality detection (signature and index computation) in the earlier linear and non-linear system analyses. The features from the transformations were plotted and clustered to formulate the signature with order variable being the exogenous demand. Index values were also computed from each feature parameter combination (two parameters used for each feature); FT Total and FT 80\% Energy, lags/max lags of 18 and 36 for ACF and CCF. Finally, the index values were computed using the three alternative index definitions in each case.

The findings from the analysis are discussed under three sub sections. First, the rogue seasonality signatures derived from alternative features are discussed to subjectively assess the best feature for this application. Next the index values derived from these features (with different parameters) are discussed. The feature parameter combinations are also compared in a structured way on the consistency and discrimination ability criteria as in chapters 4 .and 5. Finally, correlation coefficient between the index values based on the alternative index definitions is discussed. 


\subsubsection{Analysis of rogue seasonality signature derived from different features}

The profiles of variables based on FT, time, ACF and CCF features together with their clustering for signature formulation are given in Figures 6.2, 6.3, 6.4 and 6.5 respectively. All three dyads are shown in each figure for effective comparison.

The unfiltered and filtered spectra (square of FT amplitudes) plots and the hierarchical clustering plot based on the latter for each of the three dyads is given in Figure 6.2. The logic of filtering out the low frequencies (as discussed previously) is apparent from the unfiltered plot; most variables have significant energy at the low frequencies and these frequencies are seen to mask the relevant high frequencies in many cases. The filtered spectra plots of Steel works-Section mill and Steel worksBar mill dyads show rogue seasonality being generated in these systems. In the Steel works-Section mill dyad, while the exogenous demand (variable 9) shows seasonalities of only 6 and 4 months (corresponding frequencies of 0.17 and 0.25 ), other variables show an additional seasonality (rogue) of 3 months (frequency 0.33 ).

Similarly, in the case of the Steel works-Bar mill dyad many variables show additional seasonalities of 3 and 6 months (frequencies of 0.33 and 0.17 ) which is not present in the exogenous demand pattern (variable 23). In both these dyads therefore, the presence of rogue seasonality is effectively indicated; seasonal patterns in multiple variables whose frequency is different from those in exogenous demand. On the other hand in the Steel works-Rod mill dyad, all the variables are indistinguishable from the exogenous demand (variable 37 ) indicating no rogue seasonality generation in this system. Looking at the clustering profiles of the variables, it is seen that exogenous demand (variables 9 or 23) is clustered reasonably separately from the other variables in the two dyads with rogue seasonality (Steel works-Section mill and Steel works-Bar mill) and reasonably together with the other variables in the case of Steel works-Rod mill dyad which does not show rogue seasonality. The signature, which is based on the cluster profiles of the variables (see Sections 4.1.1 and 4.1.2) is therefore quite effective in indicating the presence/absence of rogue seasonality for the FT Total feature parameter combination. 


\section{Frequency domain - FT Total}

\section{MTS: Steel Works - Section Mill dvad}
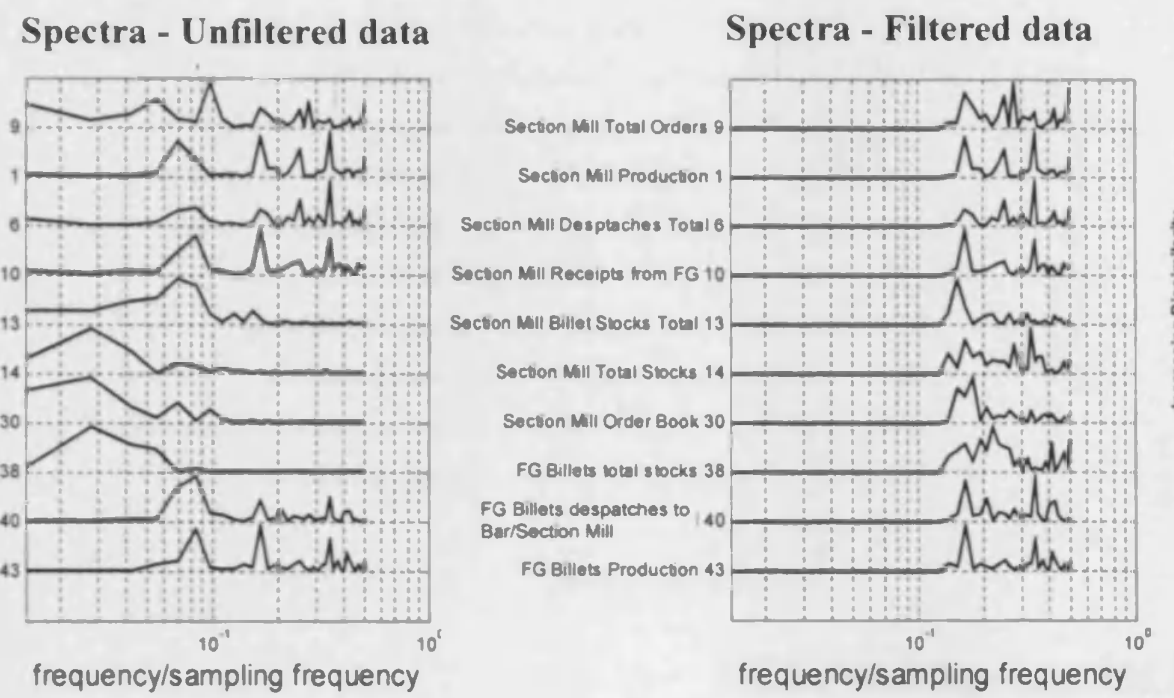

\section{Clustering of FT amplitudes}

Filtered data

frequency/sampling frequency

\section{MTS: Steel Works - Bar Mill dvad}

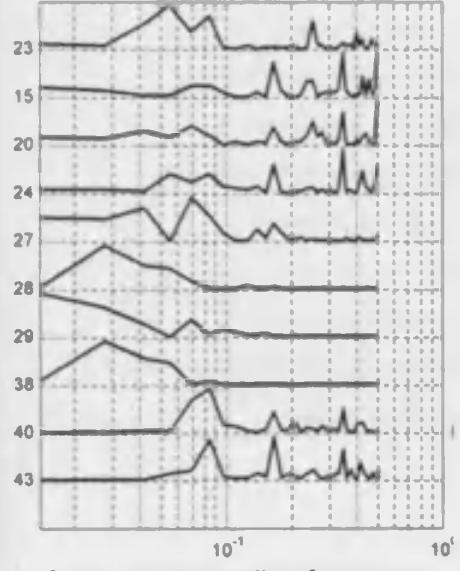

frequency/sampling frequency

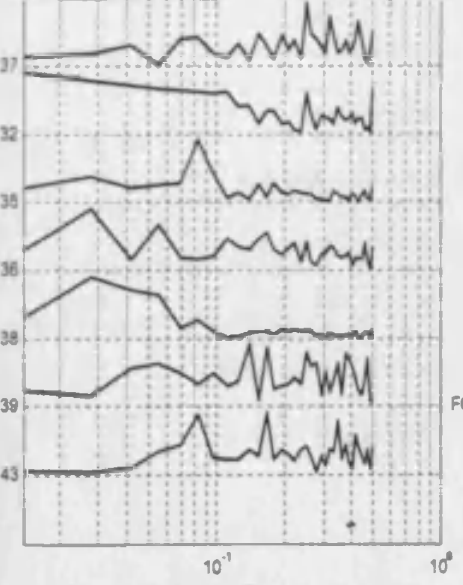

frequency/sampling frequency
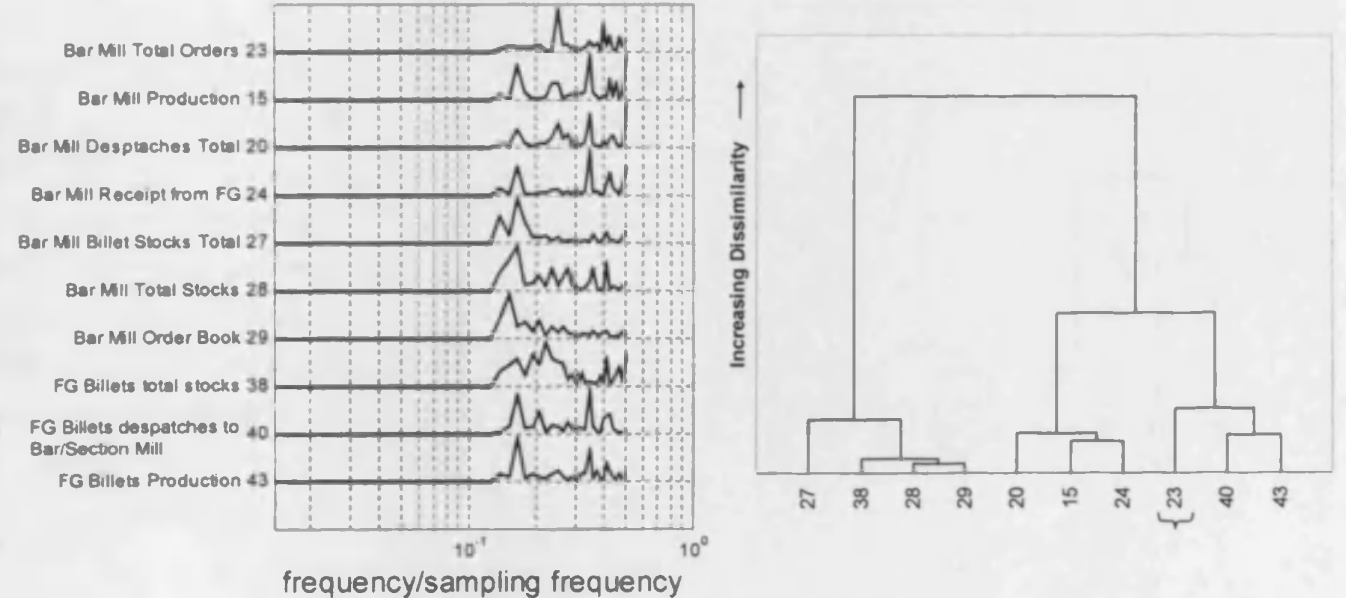

MTO: Steel Works - Rod Mill dyad
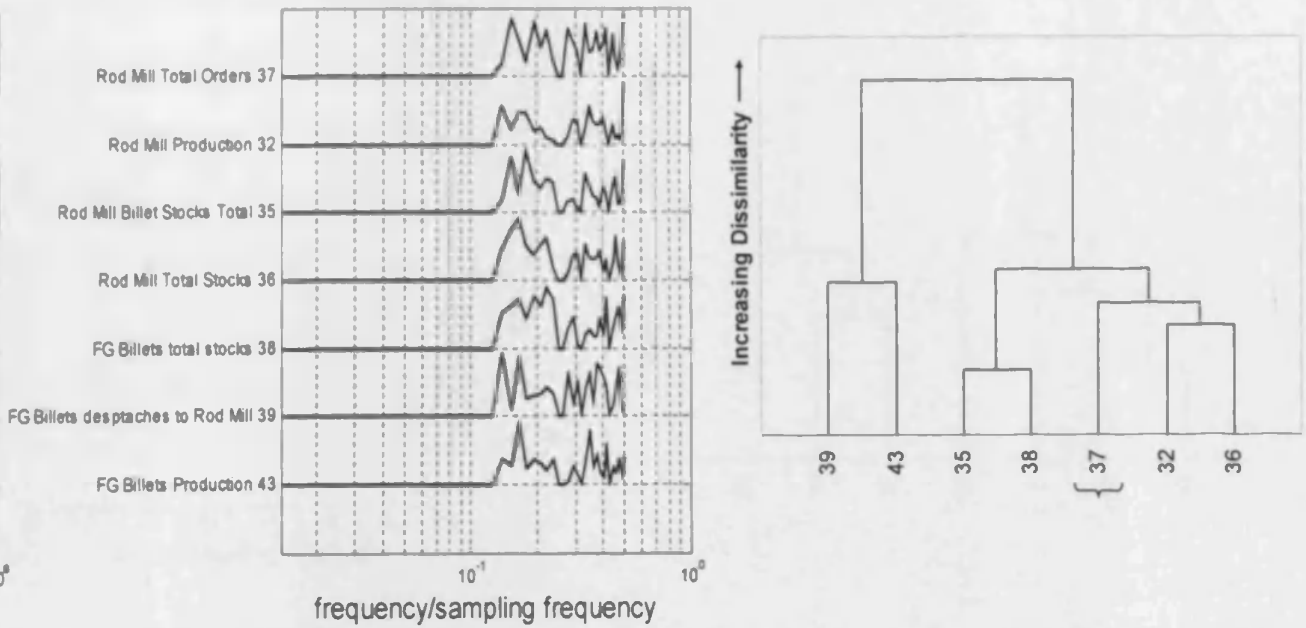

Figure 6.2. Profiles of variables in the frequency domain and clustering based on these profiles for the three dyads in the supply network 


\section{Time Domain}

\section{MTS: Steel Works - Section Mill dyad}
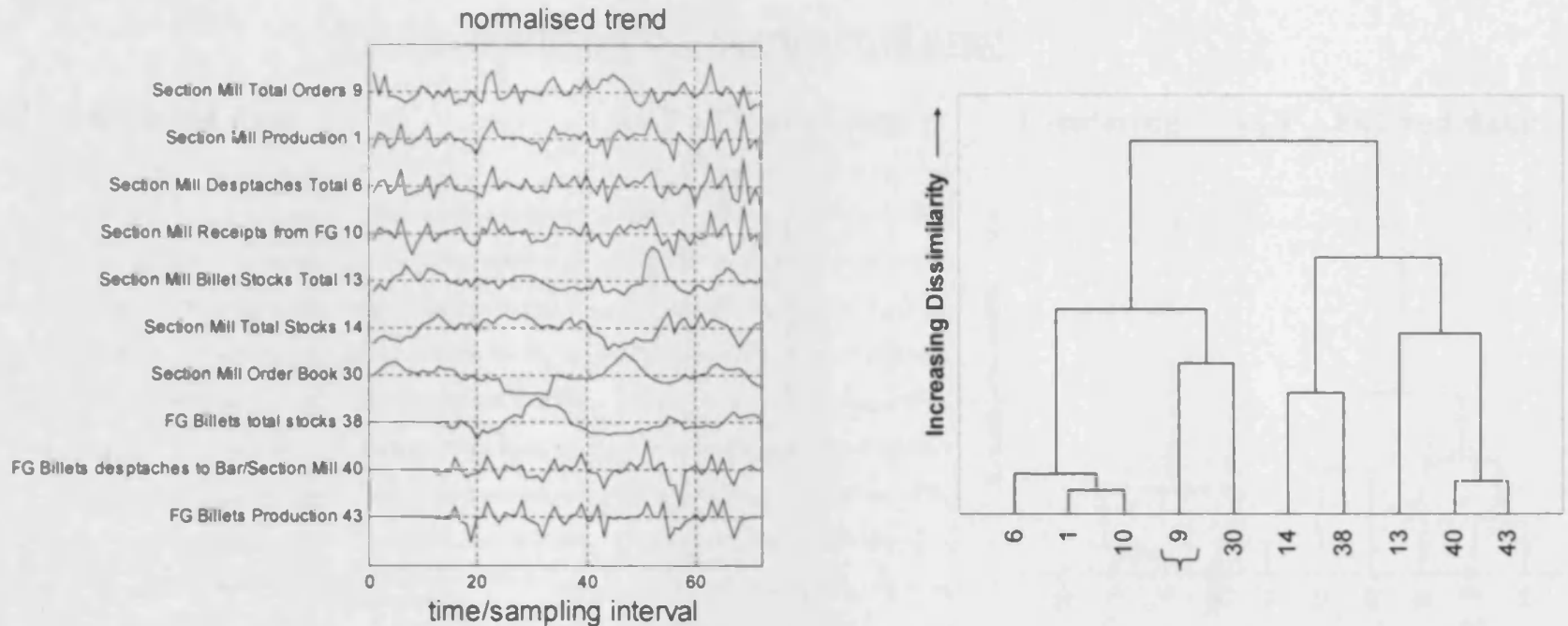

\section{MTS: Steel Works - Bar Mill dvad}
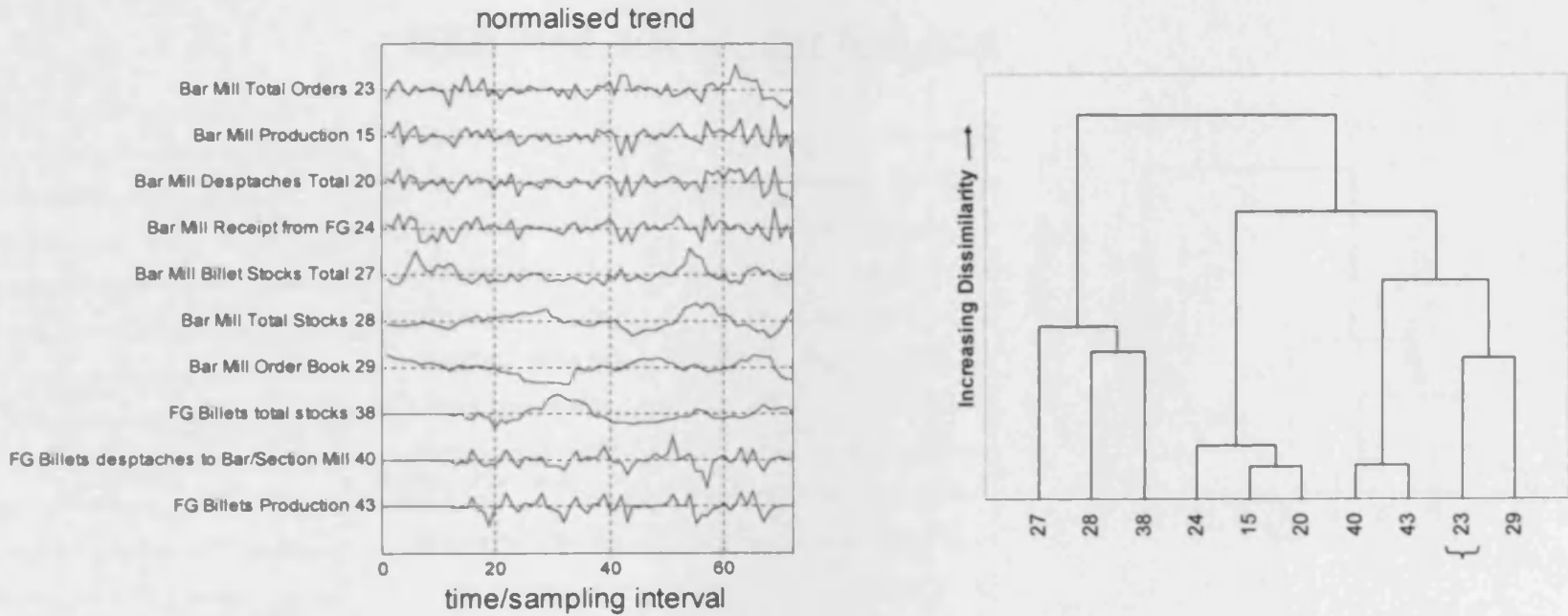

\section{MTO: Steel Works - Rod Mill dvad}
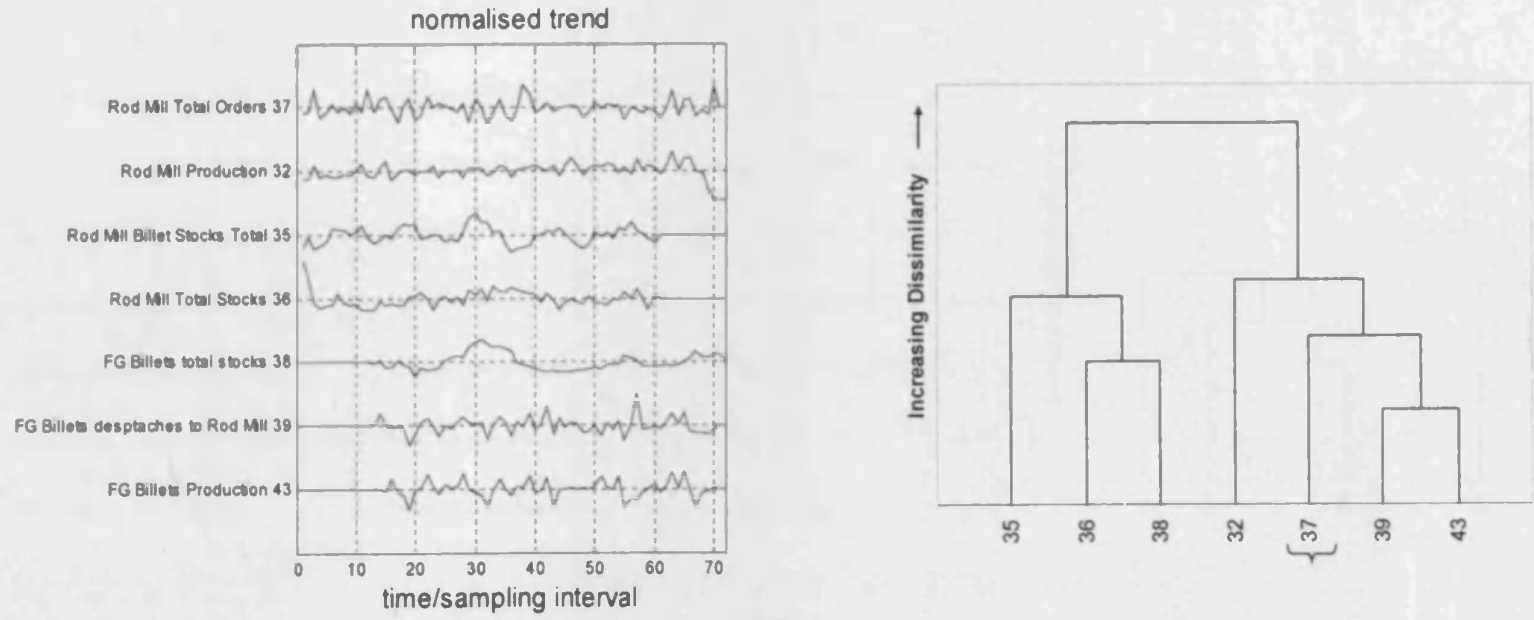

Figure 6.3. Normalised time series profiles and clustering based on these profiles for the three dyads in the supply network 


\section{Autocorrelation function (ACF) domain - Upto lag 36}

\section{MTS: Steel Works - Section Mill dyad}

\section{ACF - Unfiltered data}
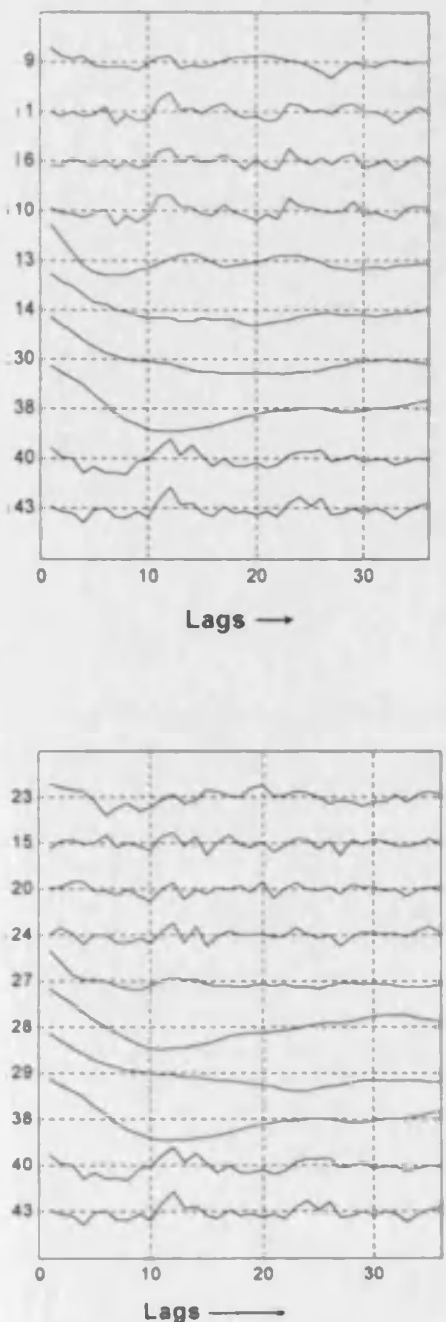

\section{ACF - Filtered data}

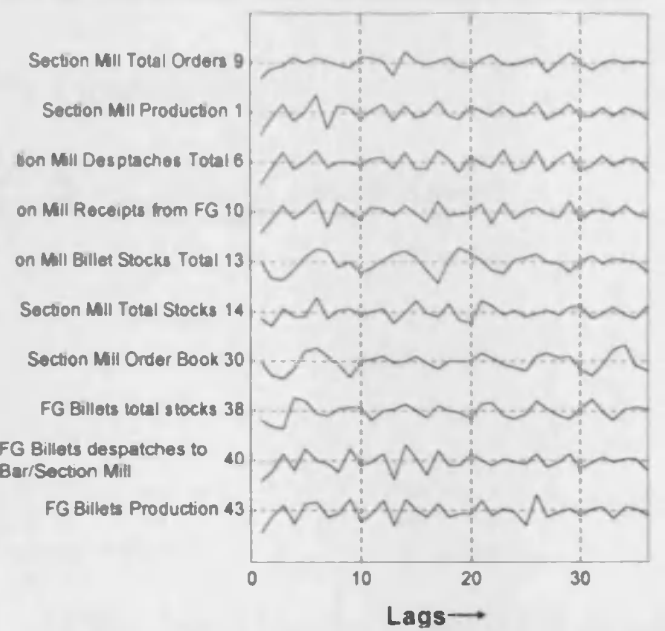

\section{MTS: Steel Works - Bar Mill dvad}

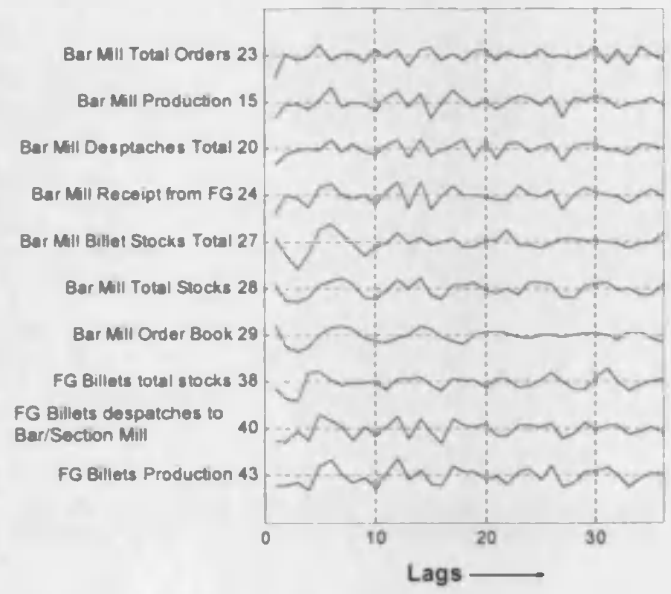

\section{MTO: Steel Works - Rod Mill dvad}
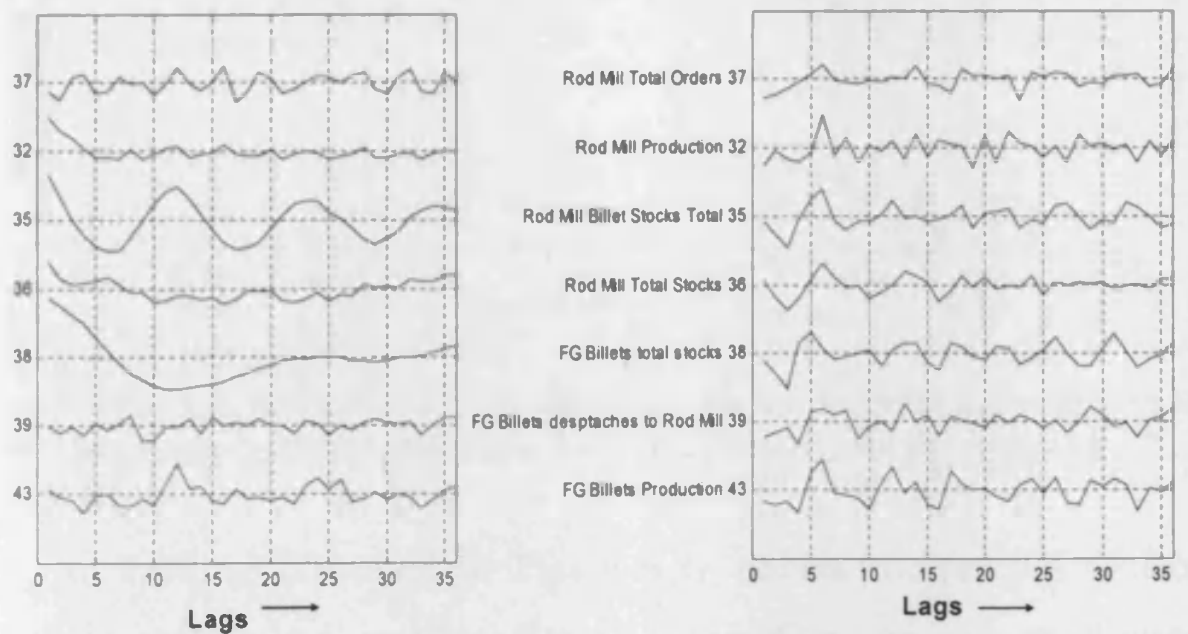

Clustering of ACF - Filtered data
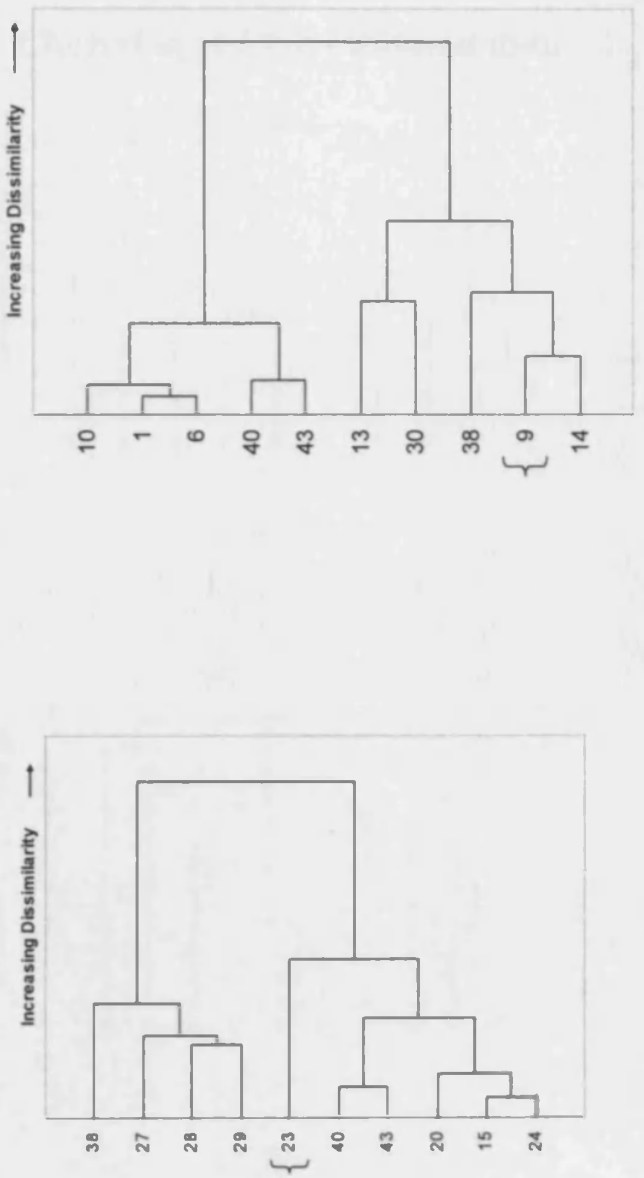

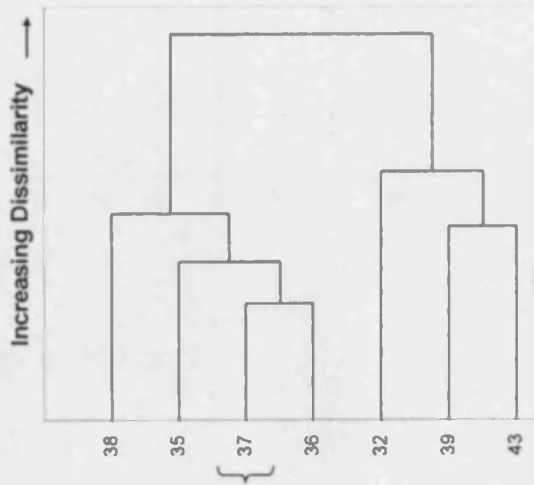

Figure 6.4. Autocorrelation function (ACF) profiles and clustering based on these profiles for the three dyads in the supply network 


\section{Cross correlation function (CCF) domain - Within lags \pm 36}

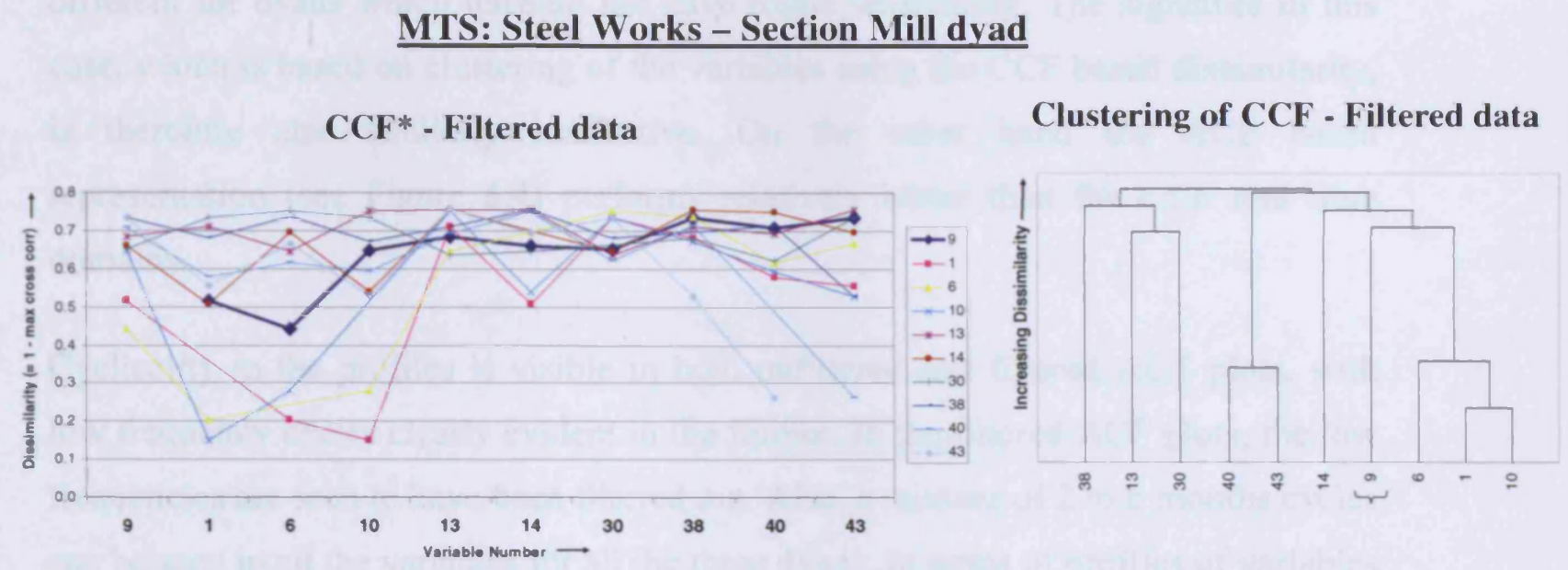

MTS: Steel Works - Bar Mill dvad
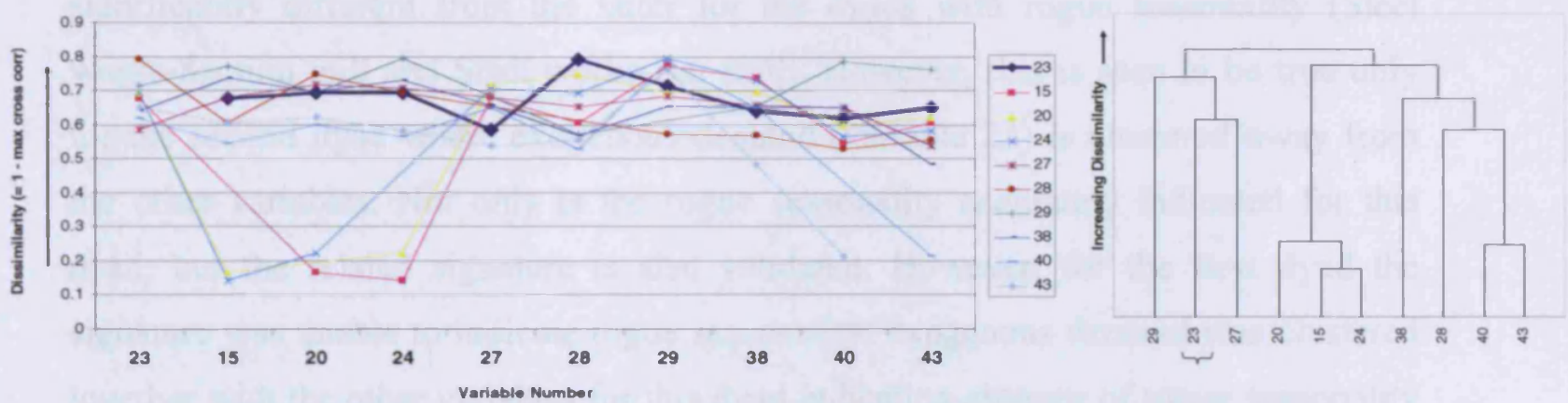

\section{MTO: Steel Works - Rod mill dyad}
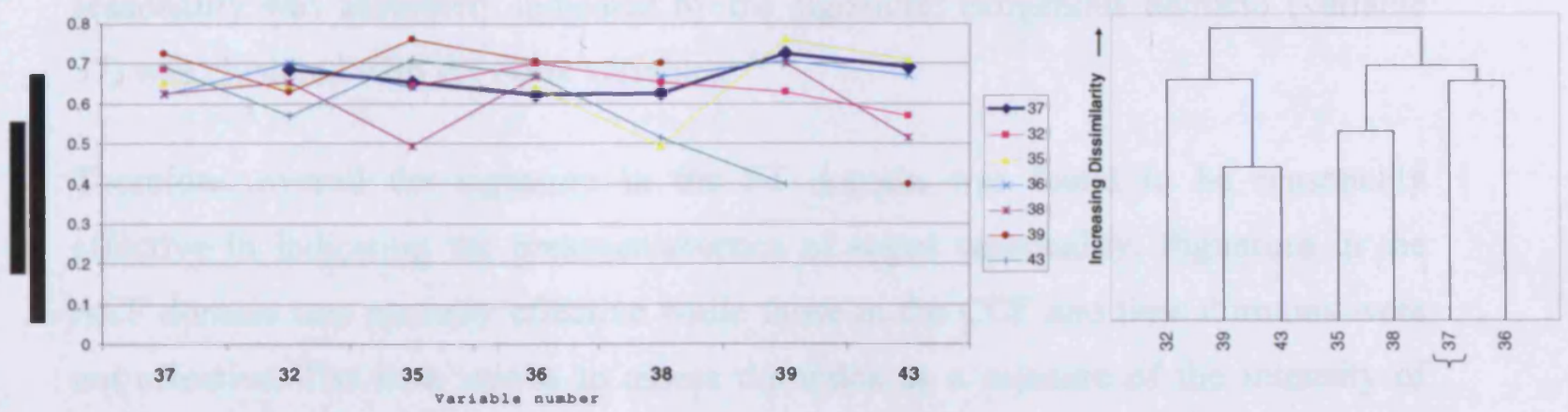

Figure 6.5. Cross correlation between variables (within maximum lags \pm 36 ) and clustering based on it for the three dyads in the supply network

In the time domain (see Figure 6.3), neither the profiles of the variables nor the signature based on clustering of the profiles are able to discriminate between the dyads which have and those which do not have rogue seasonality which was possible with the FT feature. The same is true for the CCF feature. Figure 6.5 shows that the 
dissimilarity (based on CCF) of exogenous demand with the other variables in relation to the dissimilarities among the variables themselves is not significantly different for dyads which have/do not have rogue seasonality. The signature in this case, which is based on clustering of the variables using the CCF based dissimilarity, is therefore also similarly ineffective. On the other hand the ACF based representation (see Figure 6.4) performs relatively better than the CCF and time domains.

Cyclicality in the profiles is visible in both unfiltered and filtered ACF plots, with low frequency cycles clearly evident in the former. In the filtered ACF plots, the low frequencies are seen to have been filtered out. Also, a mixture of 2 to 6 months cycles can be seen in all the variables for all the three dyads. In terms of profiles of variables in relation to exogenous demand, one would expect to see the former to be significantly different from the latter for the dyads with rogue seasonality (Steel works-Section mill and Steel works-Bar mill). However, this is seen to be true only for the second dyad where exogenous demand (variable 23) is clustered away from the other variables. Not only is the rogue seasonality accurately indicated for this dyad, but the related signature is also validated. However, for the first dyad the signature was unable to indicate rogue seasonality; exogenous demand was clustered together with the other variables for this dyad indicating absence of rogue seasonality as per the definition of the signature, though in reality rogue seasonality is present, as seen in the FT analysis. For the third dyad (Steel works-Rod mill), absence of rogue seasonality was accurately indicated by the signature; exogenous demand (variable 37) was clustered with the other variables.

Therefore, overall the signature in the FT domain was found to be reasonably effective in indicating the presence/absence of rogue seasonality. Signature in the $\mathrm{ACF}$ domain was partially effective while those in the $\mathrm{CCF}$ and time domains were not effective. The next step is to assess the index as a measure of the intensity of rogue seasonality and then use the index value for a structured comparison of different feature parameter combinations.

\subsubsection{Structured comparison of feature parameter combinations for index computation}

The index values based on equation 4.1 were computed for each dyad based on each 
feature parameter considered: time, FT Total, FT 80\% Energy, ACF 18, ACF 36, CCF 18 and CCF 36. This was done twice; first using all the variables (as given in Figure 6.1) and then using only the order and inventory variables. The different feature parameter combinations were compared in a structured way using the criteria of consistency and discrimination ability (stochastic stability criteria was not used as it is not applicable for empirical data). The consistency criteria uses the fact that the dyads operating on an MTS basis which show rogue seasonality characteristics should have a higher index value than the MTO dyad which does not show rogue seasonality (the basis for this has been discussed in chapter 4). The criteria on discrimination ability which is based on ranking of the index values is applied slightly differently here than in chapters 4 and 5. In those chapters (chapters 4 and 5), only systems with rogue seasonality (MTS and hybrid MTS-MTO) were used in the structured comparison of feature parameter combinations so a larger index value was the only basis for assigning ranks. On the other hand in the present analysis, systems with rogue seasonality (MTS dyads) are clubbed together with those without the same (MTO dyad) so that while ranks are assigned to those with greater index values in the former, in the latter (MTO) case smaller index values would better represent absence of rogue seasonality and were therefore assigned higher ranks. It is also to be noted that the structured comparison of the "all variables used" and "order and inventory variables used" cases is done together because of paucity of data. Table 6.1 gives the summary output of the analysis whose key points are:

- The index values for the MTS systems (Steel works-Section mill and Steel works-Bar mill dyads) are much lower and for the MTO system (Steel works-Rod mill dyad) much higher than those observed for MTS and MTO systems in the linear supply chain system analysis in chapter 4 . Two factors can be used to explain this. The first is that, although the dyads are being referred to as MTS and MTO systems they are actually both hybrid MTS-MTO systems (discussed at the start of section 6.2) though one is closer to the former (MTS) and the other closer to the latter (MTO). This is corroborated when the index values of these dyads are compared with those for a hybrid MTS- MTO system. For example, the index value for the simulated hybrid MTS-MTO system (see chapter 5, Figure 5.4) at 1.07 is close to the index values for the dyads as given in Table 6.1. The low index values of the dyads are in conformity with their clustering based signatures seen earlier which neither 
Table 6.1: Assessment of select feature parameter combinations used for rogue seasonality index computation in the steel case study

\begin{tabular}{|c|c|c|c|c|c|c|c|c|}
\hline & System Description & Raw Time & FT Total & $\begin{array}{l}\text { FT 80\% } \\
\text { Energy }\end{array}$ & ACF 18 & ACF 36 & CCF 18 & CCF 36 \\
\hline \multirow{3}{*}{$\begin{array}{l}\text { All avaliable } \\
\text { variables used }\end{array}$} & MTO: Steel Works-Rod MIII Dyad & 0.84 & 0.84 & 0.89 & 0.76 & 0.72 & 0.97 & 0.99 \\
\hline & MTS: Steel Works-Section Mill Dyad & 0.80 & 0.90 & 0.92 & 0.67 & 0.72 & 0.70 & 0.71 \\
\hline & MTS: Steel Works-Bar Mill Dyad & 0.79 & 0.94 & 0.96 & 0.93 & 0.95 & 0.97 & 0.98 \\
\hline \multirow{3}{*}{$\begin{array}{l}\text { Only Order and } \\
\text { Inventory } \\
\text { variables used }\end{array}$} & MTO: Steel Works-Rod Mill Dyad & 0.93 & 0.82 & 0.90 & 0.82 & 0.76 & 0.93 & 0.94 \\
\hline & MTS: Steel Works-Section Mill Dyad & 0.90 & 1.22 & 1.46 & 0.65 & 0.64 & 0.92 & 0.94 \\
\hline & MTS: Steel Works-Bar Mill Dyad & 0.87 & 1.06 & 1.21 & 1.06 & 1.01 & 0.91 & 0.92 \\
\hline \multicolumn{2}{|c|}{$\begin{array}{l}\text { Number of cases where MTS > MTO; Max possible } 4 / 4 \\
\text { (Measures consistency of index) }\end{array}$} & $0 / 4$ & $4 / 4$ & $4 / 4$ & $2 / 4$ & $3 / 4$ & $0 / 4$ & $0 / 4$ \\
\hline \multicolumn{2}{|c|}{$\begin{array}{l}\text { Sum of ranks (Row wise ranking; For MTS larger values get } \\
\text { higher rank \& reverse for MTO; Best } 8 \text {, Worst } 56 \text { ) } \\
\text { (Measures discrimination ability of index) }\end{array}$} & 42 & 23 & 24 & 30 & 23 & 40 & 42 \\
\hline
\end{tabular}

showed exogenous demand to be very distinctly clustered from other variables nor clustered very closely with them for the FT Total feature.

The second contributing factor for the significant difference in index values between the dyads in the steel case study and the corresponding simulated systems in chapter 4 is the presence of noise in the steel data as well as its varying character across the variables, which is typical of most real world datasets. In contrast in simulation, noise was only introduced in one variable (CONS or exogenous demand) with all the other variables being determined deterministically on the basis of the simulation equations. Moreover, the noise introduced in CONS was also stationary (Gaussian with a mean of 0 and standard deviation of 1 ).

Overall, the inference that could be drawn from the index values of the steel dyads is that, in reality it is unrealistic to expect supply chains with rogue seasonality to yield high index values, and those without, low index values as was observed in the analysis of linear and non linear supply systems (chapters 4 and 5). The difference in the index values between the cases when rogue seasonality is present to when it is not present would be lower in reality. Careful setting of thresholds for the same would therefore be necessary for practical situations. The relevance of the magnitude of index as a criterion for structured comparison of different feature parameter combinations (see section 4.4.1.2) also becomes apparent. 
- In terms of comparing the "all available variables used" with the "order and inventory variables used" case, the index values (based on FT Total) for the latter are marginally greater than for the former in case of MTS systems. A similar marginal difference between these cases was seen in the analysis of linear (Section 4.4.3) and non-linear (Section 5.6.2) supply chain systems. Hence, use of a limited set of relevant variables (such as order and inventory variables) instead of all the variables does not significantly impact the index value and therefore the effectiveness of rogue seasonality detection.

- The structured comparison of the feature parameter combinations based on the criterion of consistency and discrimination ability reveals FT Total and FT $80 \%$ Energy to be the best for rogue seasonality detection followed closely by ACF (specifically ACF 36 feature parameter combination) with CCF being the worst among those considered. This finding is exactly similar to that found in the simulation analysis of linear as well as non-linear (Beer game) systems.

\subsubsection{Comparison of alternative index definitions}

The index values were computed using alternative index definitions (index, Alt 1 and Alt 2) for each dyad for each feature parameter combination used. Correlation coefficient between the index values based on the three index definitions was then used to assess whether the indices were measuring the same thing. These correlation coefficient values are given in Table 6.2. The correlation coefficient is quite high for FT and ACF indicating that these features are less susceptible to changes in the index definitions and are therefore more robust for rogue seasonality assessment.

Table 6.2: Correlation between alternative rogue seasonality indices for the steel case study* data

\begin{tabular}{|l|r|r|r|}
\hline & Index and Alt 1 & Index and Alt & Alt 1 and Alt \\
\hline Time & 0.50 & 0.49 & 0.60 \\
FT & 0.95 & 0.94 & 0.97 \\
ACF & 0.96 & 0.82 & 0.79 \\
CCF & 0.28 & 0.63 & 0.69 \\
\hline
\end{tabular}

* Includes cases where all variables are used in the computation of the index as well as where only orders and inventory are used

Highlighted indicates low correlation (considered bad) 


\subsection{Discussion of the steel case study findings}

Analysis of the steel case study corroborates the findings on rogue seasonality obtained from the simulation analysis in chapters 4 and 5 . The presentation of rogue seasonality as multiple variables oscillating in sync with each other at same frequency/ies, with one or more of these oscillation frequencies not present in the exogenous demand, is validated. The signature of rogue seasonality which is, exogenous demand being clustered away from the other variables when rogue seasonality is present, is also validated. However, the validity of the signature was found to differ across the features used to derive the signature. While the FT feature showed perfect validity, validity for signature based on ACF was partial and signatures based on time and $\mathrm{CCF}$ were completely ineffective in characterizing presence/absence of rogue seasonality.

Comparison of different feature parameter combinations for computing the index revealed, FT Total to be the best followed by FT $80 \%$ Energy. These were also the best and second best in the case of simulated linear and non-linear (Beer game) supply chain systems. In the present analysis, FT feature being the best for index computation is in conformity with it giving the signature with the highest validity as discussed earlier. The use of order and inventory variables for index computation rather than all the available variables causes only a marginal change in the index values. Finally, index values based on alternate index definitions were found to be significantly correlated with each other when these were computed using FT and ACF features. Index values derived from these features are therefore robust to variations in the way these are computed.

Overall, this analysis validates the findings in Thornhill and Naim (2006). Frequency representation based on FT was found to be the best technique for rogue seasonality detection as observed by them. However, here we used an automated as well as a more generalisable approach which did not require compression of the profiles within three principal components, manual clustering and cluster evaluation and use of the look up table. Moreover, multiple techniques such as ACF and CCF besides the time domain were evaluated in the determination of FT as the best technique while Thornhill and Naim used just the time domain in the comparison. In terms of rogue seasonality in different steel units, Thornhill and Naim gave a black and white 
interpretation; Section mill and Bar mill were exhibiting rogue seasonality while no rogue seasonality was evident in the Rod mill. As against this the signature and index based analysis here suggests Section mill and Bar mills to be having a greater rogue seasonality intensity than the Rod mill. This is a more realistic analysis given that all supply chains exhibit rogue seasonality but with differing intensities (Kim and Springer, 2008).

Next rogue seasonality generation in a grocery case study is discussed whose context is different from the previous case study. First daily data, which is more noisy is used as compared to monthly data used in the steel case study. Secondly, individual SKU's are analysed rather than aggregating all the products into tons and analyzing on that basis as was done in the steel case study analysis. The rogue seasonality analysis in the grocery case study is therefore of individual SKU's in one supply chain rather than one product (aggregated in tons) in multiple supply chains as used for the steel case study.

\subsection{Description of the grocery case study context}

The grocery supply chain network used in the study is depicted in Figure 6.6. The structure of the network including the nature of material and information flows is based on discussions with the focal company's supply chain planning personnel. The flow of material (coffee) takes place as follows. The manufacturing facility of the company $\mathrm{M}$ produces coffee (in batches) which is transferred to one of its two distribution centres (DC's) (the factory keeps negligible stocks) from where it is either shipped to the large primary customers (managed by company $M$ itself) or shipped to a depot of the distributor WD who distributes goods to M's small customers, called radial customers. Some inter DC transfer of material also takes place due to misallocation or errors such as in forecasting. Distributor WD, which manages the distribution of goods to M's radial customers, is also a Wholesaler for the same set of goods. The network of depots used to distribute company M's goods (11 depots relevant to company $\mathrm{M}$ and uniquely assigned to each $\mathrm{DC}$ ) is therefore also used by WD for stocking and shipping goods to its wholesale customers. In terms of ordering practice, radial customers place their orders to company $M$ who ensures timely delivery of goods by coordinating with WD. Any shortfalls at the WD depot are met by dispatching appropriate quantity of goods from the DC so that requirements of the 

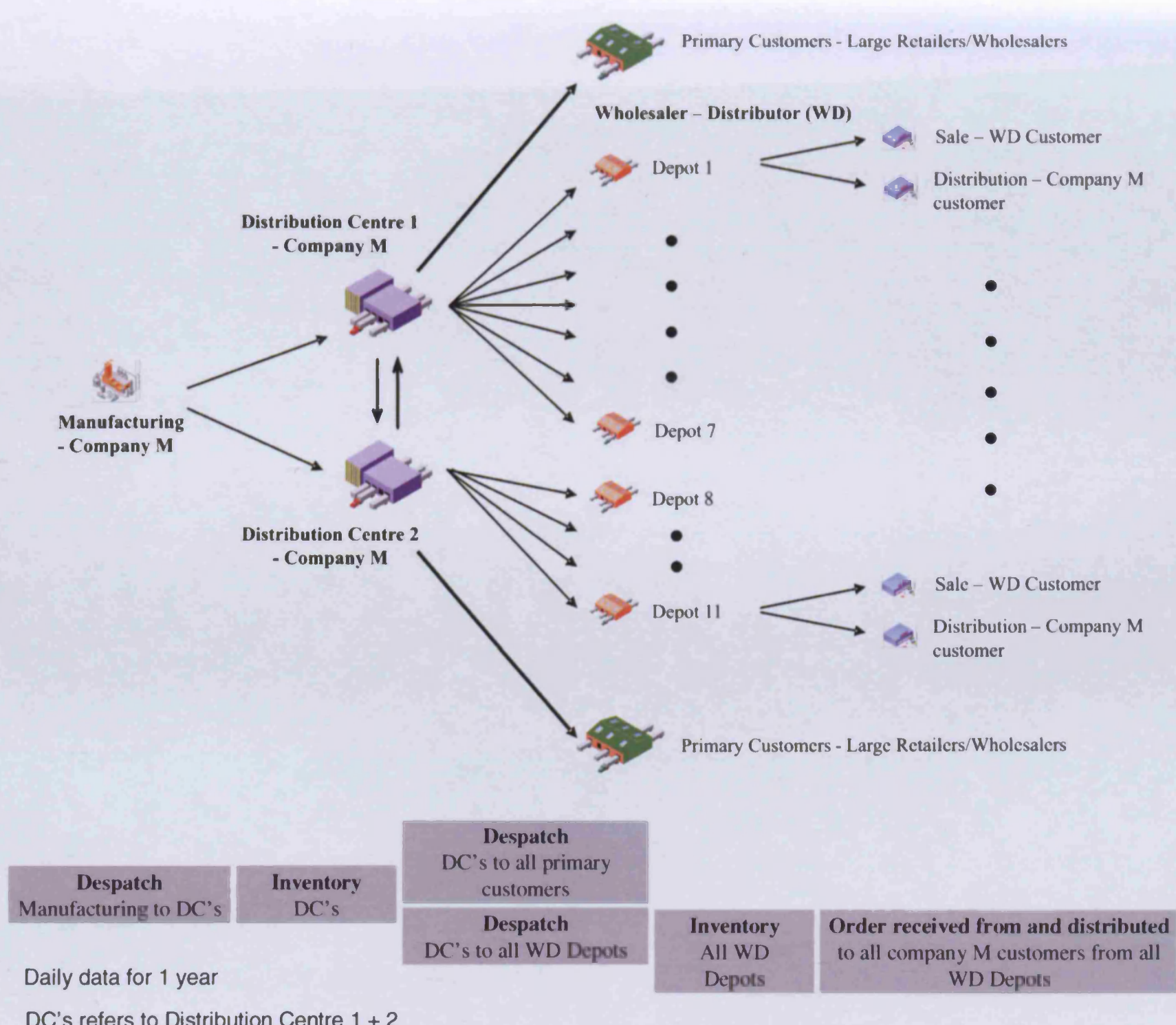

Figure 6.6 Grocery case study network structure and the variables used for the rogue seasonality analysis 
radial customer could be met. Therefore, in principle, company M's radial customers should not face any stock out situation; however in practice, the same entity (WD) managing the roles of both Wholesaler and Distributor means that there is scope for goods meant for M's radial customers getting diverted to WD's wholesale customers especially during shortage situations.

Four dynamically representative SKU's (SKU 1 to 4) were analysed for which data on system variables across the network such as orders and dispatches between entities in the network as well as the inventory level of entities was used. This data was provided on a date wise variable/entity wise basis for each SKU and required to be aligned and mapped to the structure given in Figure 6.6 for better understanding. However, such detailed, elemental level (individual DC and depot level) information is useful for analyzing operational disturbances, while our focus is on rogue seasonality, which is systemic in nature. The data fields for individual DC's and individual depots are therefore aggregated together with this aggregate data (given in Figure 6.6) used for subsequent analysis. One year's worth of daily time series data was used, the latter because initial discussions revealed all transaction times between entities to be less than a week (i.e. in days). Use of a weekly or monthly sampling frequency could have masked the embedded rogue frequencies. Next we discuss details of the data analysis.

\subsection{Rogue seasonality analysis of the grocery case study based on signature and index}

Each of the 4 SKU's was analysed using the aggregated system variables given in Figure 6.6. This involved formulating the signature and computing the index using different feature parameter combinations. The salient aspects of the analysis are summarised below:

- The time series data of different variables was first checked for consistency and adjusted for errors as well as for inappropriate recording, missing and duplicate data. Next, the data was aggregated, de-trended and normalised before being used for signature formulation and index computation. De-trending eliminates low frequencies which are irrelevant from the point of rogue seasonality but interfere in the process of signature formulation and index computation. Normalisation ensures that differences 
in scale as well as in amplification between the variables are eliminated and variables are only compared on the basis of their profiles.

- A supply network is analysed (see Figure 6.6) unlike the previous linear, nonlinear (Beer game) and steel case study analyses which were all supply chains. For example, DC to all primary customers and DC to all WD depots form a network and information on both these flows is included in the analyses. This could be done in the present context because of two factors. First, there are no allocation issues between the two flows and associated interference between their profiles as typically happens in a network structure. The high stock level at the DC's upstream from large production batches eliminated the need for allocation of material among the downstream entities and demand from both, primary customers as well as WD depots could be met simultaneously. Secondly, the transaction dynamics in both the flows are similar with time batching being similarly operative and goods needing to be despatched on a weekly/biweekly basis. Their flow or despatch profiles are therefore similar (though the volumes are different).

For the index computation, all common frequencies shared between orders and other variables reflecting transmission of exogenous demand were filtered out in line with the discussion in Section 4.4.2 as well as Section 6.2 for the steel case study. The specific frequencies that were filtered out for each SKU are:

SKU 1: Cycles of 3,7 and $>180$ days (frequencies $0.3,0.14$ and 0.005 )

SKU 2: Cycle of 7 days (frequency 0.14)

SKU 3: Cycle of 7 days (frequency 0.14)

SKU 4: Cycles of 3 and 7 days (frequencies $0.3,0.14$ )

The low frequency (cycle time $>180$ was present only in some (not all) variables in SKUs 2, 3 and 4 and hence was not filtered out as was done in the case of SKU 1. Filtering was done by replacing the values in the frequency channels (to be filtered) with zero.

- FT, ACF and CCF transformations were applied to the data, with time domain used as the reference as in the steel case study analysis. AR and DWT transformations were ignored because they were found to be the ineffective for rogue seasonality detection (signature and index computation) in the earlier linear and non-linear system analyses. The features from the transformations were plotted and clustered to formulate the signature. Index values were also computed from each feature 
parameter combination (two parameters used for each feature); FT Total and FT $80 \%$ Energy, lags/max lags of 15 and 30 (to correspond to half a month and 1 month) for $\mathrm{ACF}$ and $\mathrm{CCF}$. All the three index definitions were used to compute the index values in each of the analysed cases.

The basis for deriving the signature and index used here, is however different from that used in the previous linear, non-linear (Beer game) and steel case study analyses. In the previous analyses, rogue seasonality was generated in the echelon facing exogenous demand which was then transmitted to upstream echelons. The profiles of variables in all echelons were therefore similar to each other and different from exogenous demand. Exogenous demand was therefore used as the reference for formulating the signature and computing the index. In the present analysis involving a distribution network, the echelons have a short term transportation focus; they operate in a mode where goods are despatched as needed (analogous to MTO). All the distribution echelons would therefore have a similar profile with the only change coming at the factory echelon which is required to produce goods in batches (process nature of operations). Rogue seasonality or seasonality which is not present in exogenous demand but is endogenously generated, gets generated at the manufacturing or factory echelon. This then gets transmitted to upstream echelons (with respect to the factory) where it has an adverse impact on the cost economics. Therefore, instead of exogenous demand, despatch from factory to the DC's (representing factory activity) is the relevant reference in the present context which was used to formulate the signature and compute the index.

The findings from the analysis are discussed under three sub sections. First, the rogue seasonality signatures derived from alternative features are discussed to subjectively assess the best feature for this application. Next the index values derived from these features (with different parameters) are discussed; the features are also compared in a structured way on the criteria of consistency and discrimination ability (these criteria have been discussed in chapter 4). Finally, correlation coefficient between the index values based on the alternative index definitions is discussed.

\subsubsection{Analysis of rogue seasonality signature derived from different features}

The profiles of variables based on FT, time, ACF and CCF features together with their 


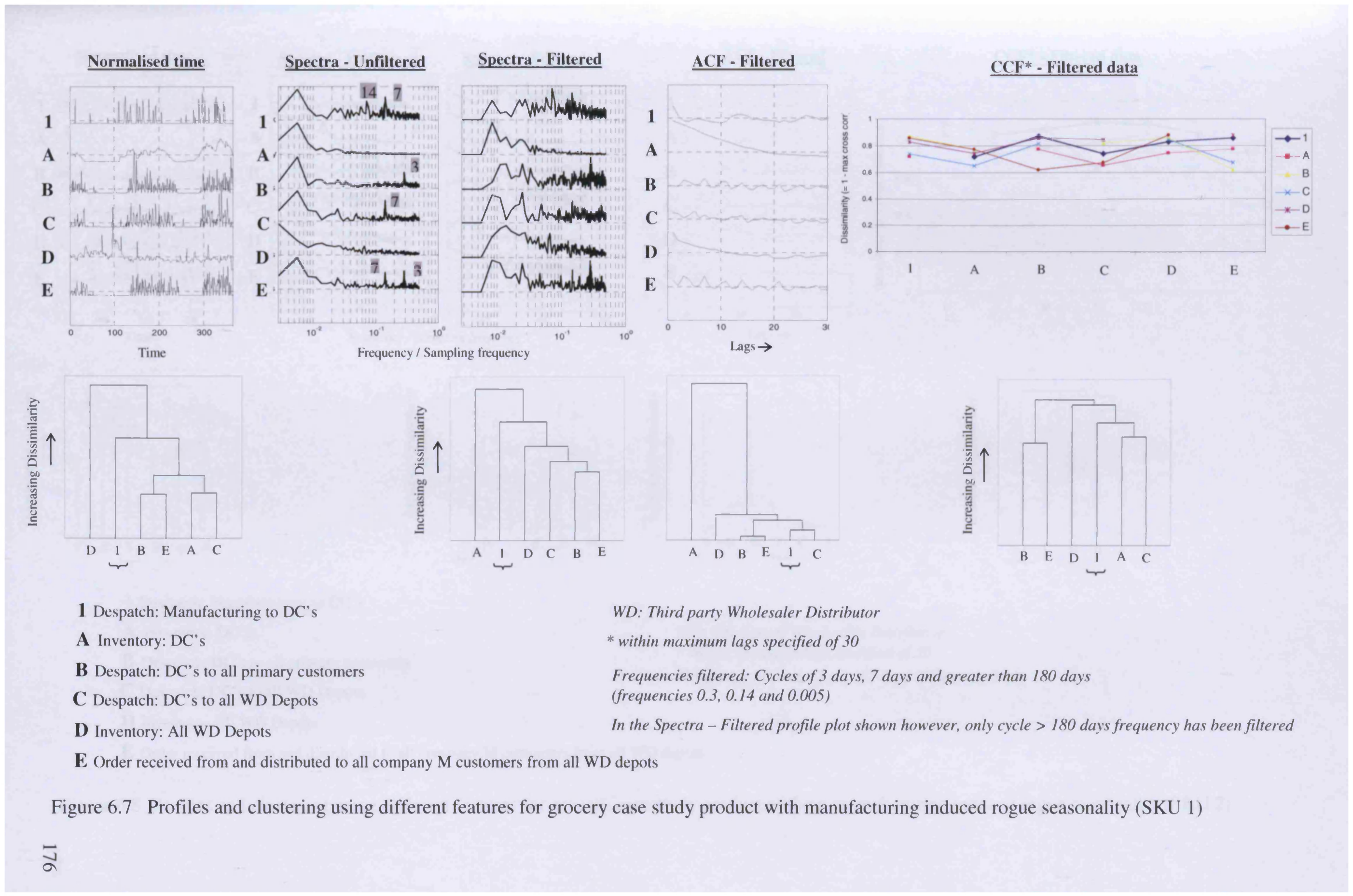




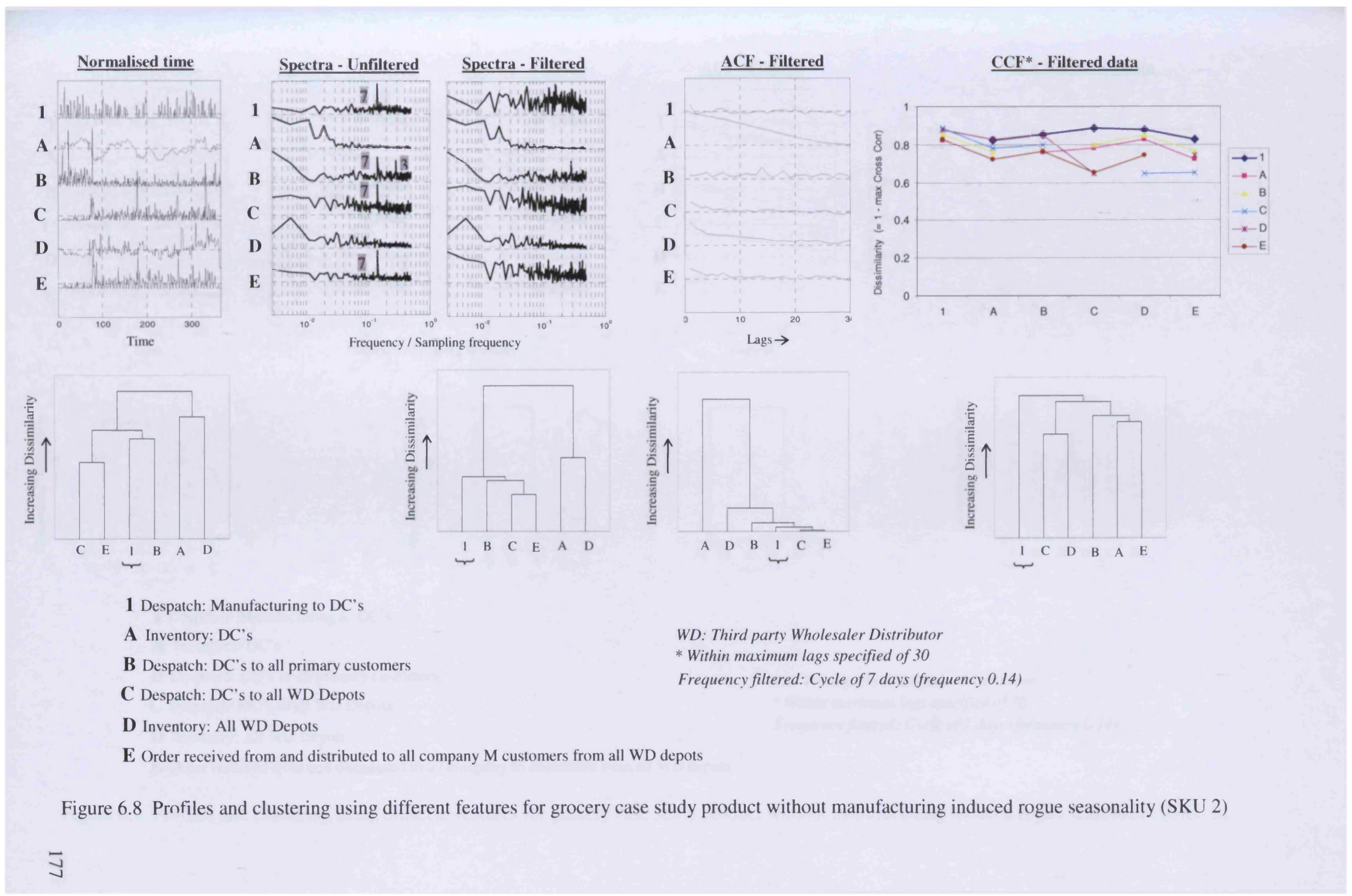



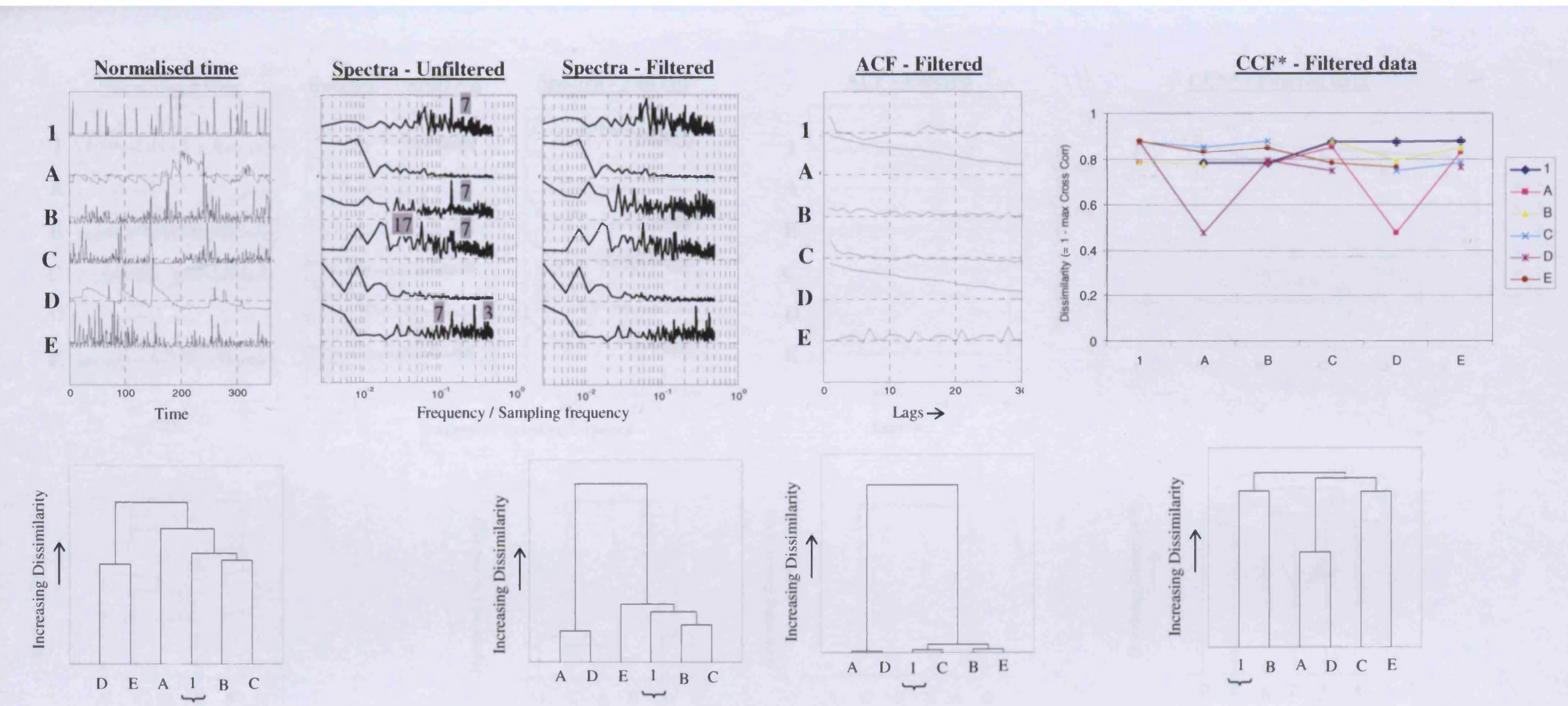

1 Despatch: Manufacturing to DC's
A Inventory: DC's
B Despatch: DC's to all primary customers
C Despatch: DC's to all WD Depots
D Inventory: All WD Depots

WD: Third party Wholesaler Distributor

* Within maximum lags specified of 30

Frequency filtered: Cycle of 7 days (frequency 0.14)

E Order received from and distributed to all company M customers from all WD depots

Figure 6.9 Prnfiles and clustering using different features for grocery case study product without manufacturing induced rogue seasonality (SKU 3) 

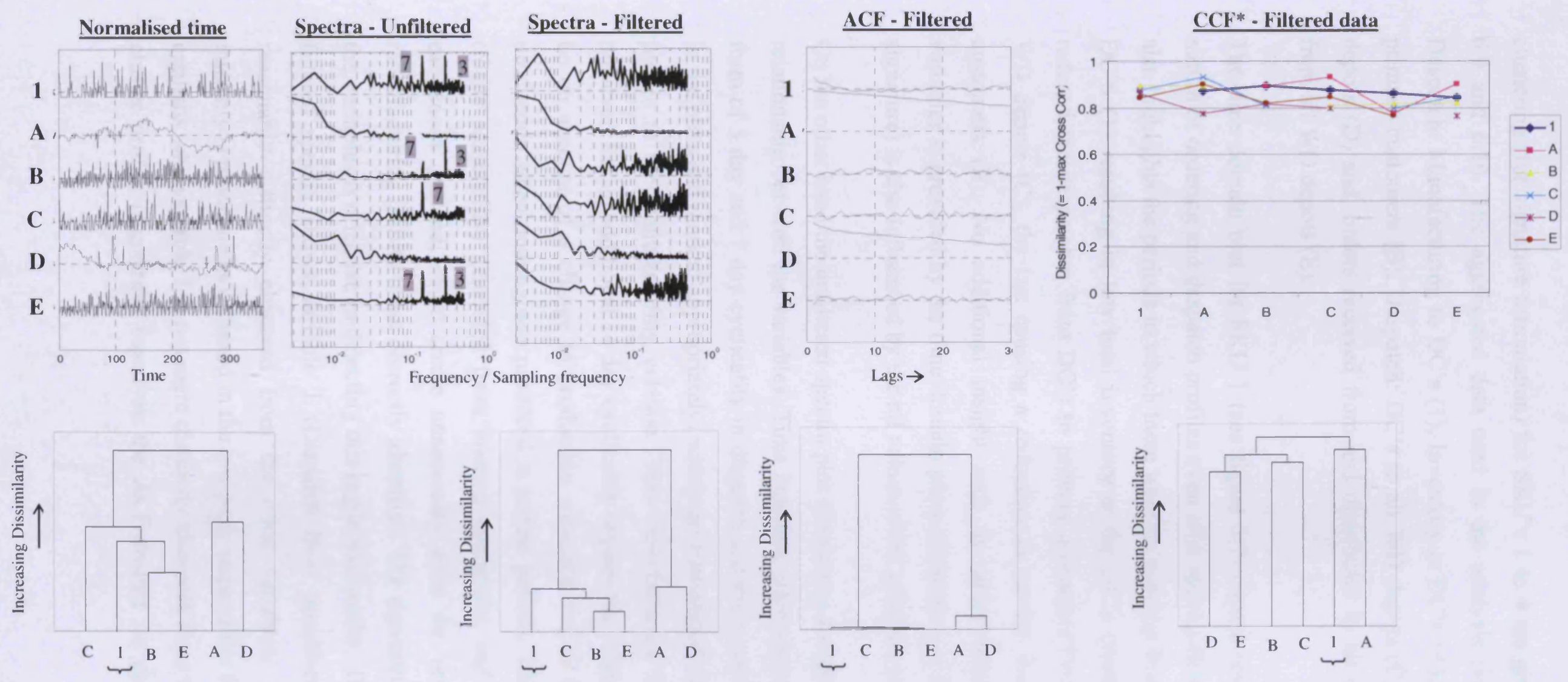

1 Despatch: Manufacturing to DC's

A Inventory: DC's

B Despatch: DC's to all primary customers

C Despatch: DC's to all WD Depots

WD: Third party Wholesaler Distributor

D Inventory: All WD Depots

* Within maximum lags specified of 30

E Order received from and distributed to all company M customers from all WD depots

Figure 6.10 Profiles and clustering using different features for grocery case study product without manufacturing induced rogue seasonality (SKU 4) 
clustering (for signature formulation) for SKU's 1 to 4 are given in figures 6.7, 6.8, 6.9 and 6.10. The aggregated data used in the analysis (in terms of cases) are: Despatch: Manufacturing to DC's (1), Inventory at DC's (A), Despatch: DC's to all primary customers (B), Despatch: DC's to all WD depots (C), Inventory at all WD depots (D) and, orders received from and distributed to all company $M$ customers from all WD depots (E).

The time domain plot for SKU 1 (see figure 6.7) clearly reveals the discontinuous nature of ordering and despatch profiles even after aggregation (1, B, C, E). This plot also highlights the periods in which there were no supplies from manufacturing to the DC'S (1) resulting in low total inventory at the DC's (variable A) which in turn reduced the despatches from DC's to primary customers (variable $\mathrm{B}$ ) as well as to WD depots (C), the last causing a reduction in service from WD depots to M's customers (E). No additional insight such as pattern/relationships between the variables is provided by the time domain plots. Clustering of the data (and associated signature) is also influenced by the nil values of the variables in many time periods.

On the other hand the unfiltered spectra plot (frequency domain) clearly highlights the relationship between the variables. Time batching effects are clearly apparent in the form of 3 day and 7 day cyclicality in dispatch and order profiles (apparent as peaks in the plots and marked appropriately) which get transmitted upstream till the dispatch profile at the manufacturing echelon. This manufacturing to DC dispatch profile however, has an additional 14 day cyclicality besides the 7 day cyclicality. This could be on account of a change in production schedule due to the earlier problems in production when nothing was produced in certain periods. This additional cyclicality (rogue seasonality) generated from internal operations, and which is unrelated to exogenous demand, could create unnecessary costs for vendors upstream of the manufacturing facility if not correctly identified. The signature based on clustering of the variables is effective in detecting this rogue seasonality. The cluster profile of the filtered spectra shows variable 1 (Dispatch from manufacturing to DC's) to be reasonably distinctly clustered from the other variables. The rogue seasonality intensity is moderate as reflected in the low peak value of the 14 day cyclicality which explains why variable 1 is not more distinctly clustered from the other variables. The cluster profile (signature) based on the ACF feature on the other hand does not 
indicate rogue seasonality at all; variable 1 is always clustered with the other variables. The same is true for the signature based on the CCF feature.

While rogue seasonality is generated in SKU 1, we next look at a contrasting case where no such rogue seasonality is generated. SKU 2 is one such case whose unfiltered spectra plot in Figure 6.8, shows time batching related cyclicality of 7 days being transmitted from downstream to upstream echelon variables and till the dispatch from manufacturing to DC's variable. However, no additional cyclicality is seen in the profile of the last variable unlike in SKU 1. The time plot of SKU 2 shows no problems in manufacturing with regular dispatches being made to DC's. No production rescheduling was required which could have created additional cyclicality as in SKU 1. The absence of rogue seasonality is accurately recorded by the signature in the frequency domain (spectra); variable 1 is clustered with the other variables. In terms of effectiveness of signatures derived from other features in capturing the absence of rogue seasonality, ACF is effective while time and CCF are less effective.

SKU 3 and SKU 4 whose profiles are plotted in Figures 6.9 and 6.10 also do not show rogue seasonality as evident from their unfiltered spectra plots. The signatures based on spectra and $\mathrm{ACF}$ are effective in representing the absence of rogue seasonality as in the case of SKU 2, while CCF and time are not.

In summary, rogue seasonality is generated in only one of the four SKU's analysed (SKU 1) with the generation taking place at the factory or manufacturing echelon and its adverse impact being felt on echelons upstream of the factory. Only signature in the FT domain (spectra) could discriminate between the presence and absence of rogue seasonality. With regards to other features, the signature based on the ACF feature correctly indicated the absence of rogue seasonality though not its presence. Signatures based on time and CCF were even less effective. The next step is to assess the index as a measure of the intensity of rogue seasonality and then use the index value for a structured comparison of different feature parameter combinations.

\subsubsection{Structured comparison of feature parameter combinations for index computation}

The index values based on equation 4.1 were computed for each SKU based on each 
feature parameter considered: time, FT Total, FT $80 \%$ Energy, ACF 15, ACF 30, CCF 15 and CCF 30. Given the limited availability of variables, the option of trying out an even lesser number for index computation was not feasible. The different feature parameter combinations were compared in a structured way using the criteria of consistency and discrimination ability (stochastic stability criteria is not relevant for empirical data and hence was not used). The consistency criteria uses the fact that the index value for an SKU showing rogue seasonality characteristics should be higher than those not showing the same while the discrimination ability criteria is based on the magnitude of the index value as discussed in previous chapters. Table 6.3 gives the summary output of the analysis.

\section{Table 6.3 Evaluation of different feature parameter combinations used for rogue seasonality index computation in the grocery case study}

\begin{tabular}{|c|c|c|c|c|c|c|c|}
\hline System Description & $\begin{array}{c}\text { Raw } \\
\text { Time }\end{array}$ & FT Total & $\begin{array}{l}\text { FT } 80 \% \\
\text { Energy }\end{array}$ & ACF 15 & ACF 30 & CCF 15 & CCF 30 \\
\hline SKU 1 - Shows rogue seasonality & 0.98 & 0.97 & 1.25 & 0.50 & 0.54 & 0.93 & 0.94 \\
\hline SKU 3 - No rogue seasonality & 0.92 & 0.81 & 0.88 & 0.28 & 0.30 & 1.00 & 1.01 \\
\hline SKU 4 - No rogue seasonality & 0.84 & 0.72 & 0.85 & 0.27 & 0.23 & 1.02 & 1.01 \\
\hline $\begin{array}{l}\text { Number of cases where Shows rogue } \\
\text { seasonality }>\text { No rogue seasonality; } \\
\text { Max possible } 3 / 3 \\
\text { (Measures consistency of index) }\end{array}$ & $3 / 3$ & $3 / 3$ & $3 / 3$ & $3 / 3$ & $3 / 3$ & $0 / 3$ & $0 / 3$ \\
\hline
\end{tabular}

- The index values (based on FT Total) are greater for the SKU with rogue seasonality than those without the same. The logic of the index is therefore effectively validated. However, the difference between their index values is low. For example, the index value for SKU 1 which shows rogue seasonality is 0.97 (not very high) while it is between 0.7 and 0.8 for SKU's 2 to 4 which do not show rogue seasonality (not very low). The case for careful setting of thresholds to detect presence of rogue seasonality discussed in the previous chapter is strengthened from these findings.

- The structured comparison of the feature parameter combinations based on the criterion of consistency and discrimination ability reveals FT $80 \%$ Energy to be the 
best followed by FT Total for rogue seasonality detection. This is in conformity with the earlier findings on signatures based on FT (or spectra) being the most accurate in indicating the presence/absence of rogue seasonality. FT features are followed by $\mathrm{ACF}$, though the index values based on the latter are quite low in the absolute sense.

\subsubsection{Comparison of alternative index definitions}

The index values were computed using alternative index definitions (index, Alt 1 and Alt 2) for each SKU for each feature parameter combination used. The correlation coefficient between the index values based on the three index definitions was then used to assess whether the indices were measuring the same thing. These correlation coefficient values are given in Table 6.4. Correlation coefficients between the index definitions were high when the FT feature was used. As against this, the other features had at least one correlation coefficient which was low.

Table 6.4 Correlation between alternative rogue seasonality indices for the grocery case study data

\begin{tabular}{|l|r|r|r|}
\hline & $\begin{array}{r}\text { Index and } \\
\text { Alt 1 }\end{array}$ & $\begin{array}{r}\text { Index and } \\
\text { Alt 2 }\end{array}$ & $\begin{array}{r}\text { Alt 1 and } \\
\text { Alt 2 }\end{array}$ \\
\hline Time & 0.95 & 0.55 & 0.65 \\
FT & 0.95 & 0.89 & 0.95 \\
ACF & 0.74 & 0.90 & 0.53 \\
CCF & 0.79 & 0.99 & 0.77 \\
\hline
\end{tabular}

Parameter options a each feature have been combined together when calculating correlation coeffcient for that feature

\subsection{Discussion of the grocery case study findings}

Rogue seasonality generation in a grocery (soluble coffee) network was investigated using the data on system variables for four SKU's. Rogue seasonality was found to be generated in $1 \mathrm{SKU}$ and at the manufacturing echelon. Long production stoppages caused rescheduling of production at the manufacturing echelon resulting in the generation of an additional 14 day seasonality which was not present in any variable in any downstream echelon including exogenous demand. Because rogue seasonality was generated at the manufacturing echelon and all downstream echelon variables were synchronized with the exogenous demand, that is, operating in a MTO fashion, the original signature and index of rogue seasonality based on exogenous demand as 
the reference required revision. Instead of exogenous demand, dispatch from manufacturing to the DC's was used as the reference.

The modified signature (clustering based on the new reference) was formulated using alternative time series transformations (and features) and assessed in terms of its effectiveness in detecting the presence/absence of rogue seasonality. The FT feature (spectra) was found to be most accurate in the same. Analysis of index values revealed the index value for SKU with rogue seasonality to be higher than each of the other SKU's without rogue seasonality (based on FT features). However, the difference in value between systems with rogue seasonality to those without it to be small, thereby strengthening the case for fixation of appropriate thresholds for rogue seasonality detection. Comparison of different feature parameter combinations for computing the index revealed FT $80 \%$ Energy to be the best followed by FT Total. The FT based index values computed using alternative index definitions were also highly correlated with each other.

\subsection{Conclusions}

Analysis of rogue seasonality in two empirical contexts in this chapter validated the simulation analysis findings. FT features were found to be the best for signature and index computation and therefore for rogue seasonality detection. Also, use of a subset of the available variables but representative variables was equally effective in rogue seasonality detection. The empirical analysis, however, also informed us about some of the difficulties in applying these findings in real environments especially in an automatic mode.

Both the steel and grocery case studies validated the characteristic manifestation of rogue seasonality as multiple variables oscillating in sync with each other. However, they differed in terms of the signature and index to be used for detecting this seasonality. While in one case exogenous demand was used as the reference for deriving the signature and index, in the other case, where manufacturing echelon was responsible for generating the rogue seasonality, dispatch from the same was used as the reference. Choosing appropriate references for different supply chains is therefore an additional pre processing step that would be required before operating the index based automatic rogue seasonality detection approach. The empirical case study 
analysis also highlighted the difference in index values between systems with rogue seasonality and those without it to be not very large. This requires thresholds to be appropriately specified so that systems with different rogue seasonality intensities could be discriminated. 


\section{Chapter 7 - Discussion and Conclusions}

This chapter presents an overview of the research findings in relation to the research questions presented in Chapter 1. After a brief review of the research background and process used, these findings are discussed in terms of their contribution to theory and practice. The limitations of the research are then explained together with avenues for further study.

\subsection{Research background and process}

This thesis has studied rogue seasonality, which is observed in many real world supply chains and reduces the cost efficiency of operations but has received only a limited academic interest. The focus is on its detection, specifically, in identifying supply chains with high rogue seasonality intensities so that timely and appropriate mitigative action could be initiated. Such an approach, which comes under the sense and respond management philosophy, has been applied in different disciplines but not in supply chain management.

Application of the sense or detection requires information about the system, techniques which use this information to detect a problem or anomaly, and relevant contexts to test and establish the effectiveness of the process. This rogue seasonality oriented study used time series information on operating variables such as orders, inventory, dispatches, shipments and backlogs with a separate analysis based on the use of only order and inventory variables. Use of the signature based technique was explored for detection, with the signature formed by clustering of these variables represented in alternative domains (and relevant features) such as time, amplitudes of FT, ACF, CCF, coefficients of DWT and coefficients of AR model. The effectiveness of the detection process was assessed on different rogue seasonality contexts, such as a simulated three echelon linear supply chain, a simulated four echelon non-linear (Beer game) supply chain and two empirical supply chains. Such a multi-context analysis gives greater generalisability to the findings and was therefore used. Since each of these contexts is significantly different from each other, they have been classified as a separate research question. 


\subsection{Discussion on research questions}

\section{Research question 1}

What signature and index could be used for detecting rogue seasonality in a linear supply chain? What is the best feature (domain representation) and the appropriate system variables for deriving them?

This question was addressed in Chapter 4 by analyzing a three echelon linear system. Rogue seasonality was seen to manifest itself as multiple system variables oscillating in sync, with exogenous demand not exhibiting this oscillatory profile. This characteristic presentation of rogue seasonality was captured in its signature which is defined as follows. If the exogenous demand variable is not clustered with other variables such as orders, inventory and work in process then rogue seasonality is considered present, otherwise not. The signature, defined in this way is a little subjective and therefore a numerical index was also defined for rogue seasonality which is based on comparing the dissimilarities amongst the variables in relation to the dissimilarity between these variables and the exogenous demand. While only the presence or absence of rogue seasonality is indicated by the signature, the index specifies the intensity of rogue seasonality in the relevant supply chain system

The index was evaluated on different configurations of the linear system, and found to be a reliable and accurate indicator of rogue seasonality intensity with systems with no rogue seasonality, moderate rogue seasonality intensity and high rogue seasonality intensity giving index values of 0 , greater than 0 and significantly greater than 0 respectively. Alternative definitions of the index based on use of average instead of minimum dissimilarity (between exogenous and the other variables) and dissimilarities expressed in terms of standard deviation were found to be strongly correlated with each other implying their robustness as a measure and that any of them could be used in practice. The index is also effective for the case of exogenous demand having seasonality but requires this seasonality to be identified on the basis of its common occurrence in demand and other variables and filtered out before computation of the index. 
Among the different features evaluated, amplitude of Fourier transform (FT) was found to be the most appropriate for signature formulation and index computation and therefore for rogue seasonality detection. Index values based on the FT feature varied with the rogue seasonality intensity as expected (high consistency) and were effective in discriminating rogue seasonalities of different intensities (discrimination ability) while being stochastically stable. Within FT, the option involving use of information in all the frequency channels was found to be the best. The second option, in which the number of frequency channels used is based on the distribution of energy among these channels was close to the previous option on all the assessment criteria (for the $80 \%$ energy option used). With regard to use of variables, using only order and inventory variables rather than all the available variables did not cause a significant change in the index value. The index is therefore robust to choice of variables as long as the behaviour of these variables is not totally inconsistent with the behaviour of the other variables.

\section{$\underline{\text { Research question } 2}$}

Can the signature and index identified from the linear supply chain be applied to a non-linear supply chain with backlogs and batching? Is the best feature and appropriate variables for detecting rogue seasonality in this non-linear system the same as in the linear system?

This question is aimed at assessing the validity of the findings obtained from RQ 1 on a non-linear supply chain with backlogs and batching. This question has been addressed in Chapter 5.

The system used for answering this question not only used backlogs and batching but also had a greater number of echelons (four) and a greater number of variables, including the shipment variable. Configurations with different levels of batching and backlogs were analysed with fill rate going down to $80 \%$ in certain cases.

The signature discussed in RQ 1 was assessed on this system and found to be effective, though there was a minor difference in the nature of the signature compared to that in the linear system. While all the other variables were clustered together and separate from 
exogenous demand in the linear case, in the present case one variable continued to be clustered with the exogenous demand. Though this occurrence is associated with a nonlinear system, it could occur in any multi-echelon system having a large number of variables. To incorporate the above possibility, the rogue seasonality signature definition was modified as follows. Rogue seasonality is considered present if the exogenous demand is separately clustered from most rather than all the variables. The value of 'most' would need to be defined which would be on the basis of the total available system variables as well as the desired sensitivity of detection.

The index value accurately reflects changes in the rogue seasonality intensity as in the case of the linear system. However, the index was less consistent in absolute terms which can be explained as due to the simultaneous influence of backlogs and batching in a multi echelon system. In terms of the features and parameters for index computation, amplitudes of FT with all frequency channels being used was found to be the most appropriate as in the linear case with frequency channels based on $80 \%$ energy the second best. The correlation between the index values based on alternative index definitions was high for most of the features and use of order and inventory variables gave the same overall findings as when all the variables are used. Both these findings are similar to that for the linear system. Overall, almost all the findings for the linear system are found to be valid for the non linear system. In the next question, the validity of these findings are assessed in empirical contexts.

\section{Research question 3}

Can the signature and index be used for detecting rogue seasonality in real supply chains? Are the best features and system variables for deriving the signature and index the same as found in RQ 1 and $R Q 2 ?$

Two empirical rogue seasonality contexts, one in steel and the other in the grocery sector, were used to supplement the findings from linear and non-linear simulation. This question is answered in Chapter 6. 
The steel case study validated the characteristic presentation of rogue seasonality observed in the analysis of simulated systems, which is, multiple variables oscillating in sync with each other at similar frequency/ies, with one or more of these oscillation frequencies not present in the exogenous demand. The signature, which was derived from the amplitudes of FT and using all the frequency channels was found to be effective in indicating the presence or absence of rogue seasonality. Similarly, the index was able to discriminate systems with high and low rogue seasonality intensities. However, the difference in index value between the systems with high and low rogue seasonality intensity was found to be low and much lower than that observed in the case of linear and non-linear systems. This highlights the need for careful setting of thresholds so as to categorise systems with different rogue seasonality intensities accurately. The findings on the best features and parameters to be used for signature and index, the relationship between alternative index definitions and regarding use of order and inventory variables instead of all the variables are similar to that for the linear and non-linear systems.

The grocery case study identifies and analyses a different rogue seasonality context than that discussed in the simulations and steel case study. Rogue seasonality generation is analysed at the SKU level which was done for 4 SKU's. Rogue seasonality generation was observed in only one SKU (SKU 1) and it was generated not in the echelon facing exogenous demand as in the case of previous simulated systems or systems in the steel case study, but further upstream in the manufacturing echelon. While all the echelons downstream of the manufacturing echelon for this SKU do not show any rogue seasonality as they operate on a time batching basis, upstream echelons are expected to show rogue seasonality as it would be transmitted to them from the manufacturing echelon. The definitions of signature and index, which assume the presence or absence of rogue seasonality characteristics across the whole system rather than parts of the system therefore need to be revised. Instead of exogenous demand as a fixed reference, the reference needs to be flexible so as to be relevant for the different nature and origins of rogue seasonality. For example in the case of SKU 1, rather than exogenous demand, a more relevant reference for deriving the signature and index is a variable at the manufacturing echelon (Dispatch from manufacturing to DC's) which is therefore used. 
Three other SKU's which did not exhibit rogue seasonality were used for comparison with the same reference as above, used in each case.

The signature and index with the modified reference were found to be effective in discriminating cases with rogue seasonality (SKU 1) to those without them (SKU 2 TO 4). However, the difference in the index value between the two cases (SKU 1 and SKU 2 to 4 individually) was small, which strengthens the need for fixing appropriate thresholds as proposed in the case of the steel analysis also. In terms of the features and parameters to be used for deriving the signature and index, amplitudes of FT and using all the frequency channels as well as those with $80 \%$ of the signal energy were found to be the most appropriate as in the case of all the previous contexts. The FT based index values computed using alternative index definitions were also found to be highly correlated with each other.

The answers to the first three research questions can be summarized as follows:

- The signature based on variable cluster profiles is effective in detecting the presence/absence of rogue seasonality with the signature derived from the amplitudes of FT and using all the frequency channels or as many frequency channels that have $80 \%$ of the signal energy, both of which are similarly effective. The definition of the signature needs to be flexible, so as to accommodate similar minor inconsistencies as seen in RQ 2, that may be found in the real world data. Also, the reference used for deriving the signature needs to be based on the nature and origin of rogue seasonality as seen in RQ 3. Though exogenous demand may still be the most relevance reference in a majority of cases, it is important to confirm the same at the outset.

- The index was found to be an accurate and robust indicator of the rogue seasonality intensity in a supply chain with a higher value reflecting a greater intensity. It is robust because multiple variables used in its computation make it less susceptible to inconsistencies in some variables. The index needs to be computed from amplitudes of the FT of the variables, with similar features being used as discussed for the signature above. Also, a similar requirement and caution regarding the reference used apply, as for the signature. Among the three index definitions proposed, any of them 
could be used for computing the index, as index values based on them were found to be strongly correlated with each other when FT features are used. However, since index and Alt 1 (based on equations 4.1 and 4.3) make more intuitive sense they should be preferred. Finally, specifying threshold/s for the index values so as to classify rogue seasonality intensity as high or low is important as this would form the basis for management attention. For the simulation system, this was easy as a system without rogue seasonality yielded a 0 index value which could be used as a baseline. However, in real systems, the difference in index values between systems with low rogue seasonality and high rogue seasonality is not very large and therefore the process of establishing thresholds is quite important.

- Use of order and inventory variables instead of all the available variables was found to not significantly impact the index value. Both the options are relevant. While the former is relevant as information on other variables may not be available and computation and interpretation is easier, the latter provides stability and robustness to the index.

Given this knowledge about detecting rogue seasonality at a technical level, the next task is to exploit this knowledge at a managerial level to manage rogue seasonality. This takes us to the research question 4 .

\section{Research question 4}

\section{How can rogue seasonality detection be used in a managerial framework?}

Figure 7.1 shows the chart which specifies how rogue seasonality could be managed using the index seasonality index. While Thornhill and Naim (2006) gave a conceptual framework for such an application, this thesis strengthens it by providing a quantitative and automated basis. The scenario envisaged in Figure 7.1 is one in which many multi echelon supply chains are being managed by a focal company. This is a realistic scenario given the many examples of such centralized monitoring of supply chains such as Cisco's eHub initiative (Grosvenor and Austin, 2001) and initiatives by Eaton Corporation (Supply \& Demand Chain Executive, February 2006) and Honda (Ward's Autoworld, 
July 2006) to operate supply chain wide systems for advance warning of potential problems.

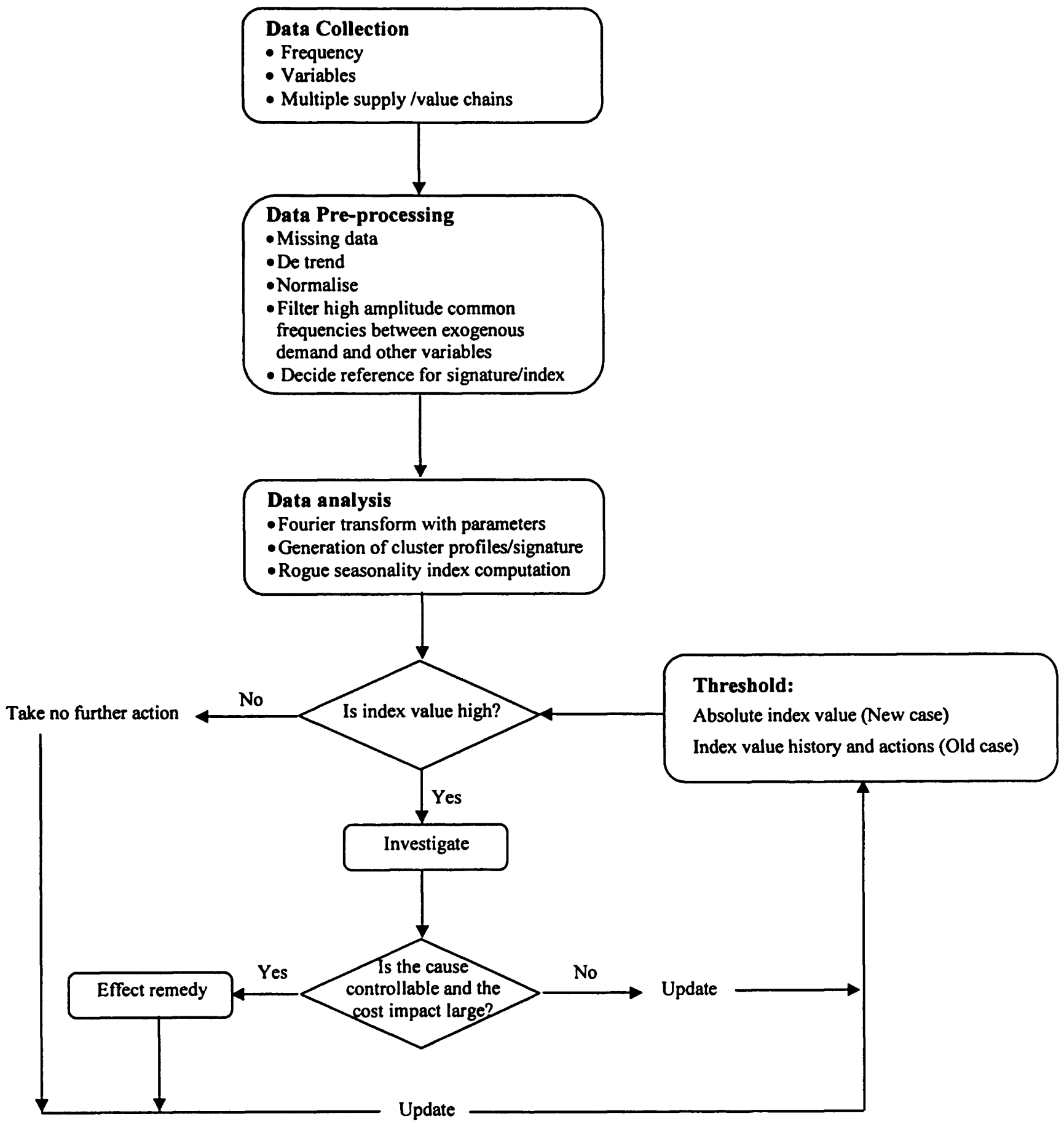

Figure 7.1 Flow chart for the practical application of the rogue seasonality index 
The first step is to decide the supply chains whose rogue seasonality intensity would be detected and managed, the information that would be available and used given the nature of relationship between players in the supply chain and the nature of dynamics in the supply chains. The last point ensures that the choice of sampling frequency for system variables is appropriate in relation to the dynamics. For example, monthly data was adequate in the steel case study analysis while the grocery case study analysis required daily data on supply chain variables. Next, the time series information of different variables is processed so that their profiles could be compared. Most of the processing steps mentioned have been applied in the steel and grocery case study analyses earlier. An important addition is the reference variable to be used for signature and index computation which could be decided on the basis of an initial sample data analysis.

The time series data of variables represented in terms of the FT feature is used to create the rogue seasonality signature and index. Application of the index requires the specification of thresholds which could be used to classify rogue seasonality intensities appropriately for management action. The following approach is proposed for the same.

A common index threshold could be specified for all newly added supply/value chains which is kept low enough to classify relatively more supply chains as having high rogue seasonality and investigated. The investigation could involve assessing the cost implications of rogue seasonality. For example, a supply chain with a high index value, could have surplus capacity and/or high flexibility, meaning less adverse cost impact from rogue seasonality and therefore not needing management intervention. The second aspect of assessment could be on the causal factors and the extent to which those could be controlled to minimise rogue seasonality. For example, a low level of trust among the focal company and its partners could make changes such as ordering heuristics difficult. Once sufficient history of index values and related management interventions is available, specific maximum thresholds for each supply or value chain could be prescribed for regular monitoring and detection of significant deviations. Since, the index does not carry information on the profiles of the variables, the signature may be used to complement it wherever required. 
The index is therefore proposed to be used not only for detecting rogue seasonality but also for assessing changes in its characteristics over time, akin to statistical process control (SPC) in a manufacturing environment. Use of multiple variables in the index's computation reduces its variability and therefore makes it easier to specify robust thresholds. Once an instance of high rogue seasonality is detected, actions such as those proposed by Kim and Springer (2008) could be effected. This would mean either decreasing the supply lead times $\left(\mathrm{T}_{\mathrm{p}}\right)$ and/or increase the time to adjust inventory ( $\mathrm{Ti}$ ) and/or reduce the time to adjust WIP $\left(T_{w}\right)$ all of which reduce the propensity of strong cyclicality. Since, these prescriptions are based on a dyadic setting, an alternative and better approach would be to work with the entities in the problem supply chain and design specific policies to reduce rogue seasonality.

\subsection{Research contributions}

This research investigated the generation of rogue seasonality in different contexts and defined the decision elements to detect and manage it. The detection process involved clustering of time series data of variables represented in alternative domains. The major contributions of this thesis are in the following areas.

Understanding of rogue seasonality: Rogue seasonality has not been treated separately from the Bullwhip effect in most studies (Forrester, 1961; Miragliotta, 2006) and the few studies that have done so, have not investigated it comprehensively. For example, Kim and Springer's (2008) study used only a dyadic structure, did not consider the impact of forecasting policies, analysed the dynamics of only two variables (inventory and pipeline stock) and used an inappropriate definition of rogue seasonality based on frequency and which was also subjective. The other study on rogue seasonality by Thornhill and Naim (2006) is empirically oriented but similarly limited in terms of analysis.

This thesis carries out a comprehensive and rigourous investigation of rogue seasonality. Studies of this nature have been done on the Bullwhip effect but not on rogue seasonality. The study provides understanding about the impact of different demand processes and parameters, delays, order of delays, control policies, linear and non-linear structures and 
batching on generation of rogue seasonality. Empirical contexts, which were of the multi echelon kind, were also analyzed from the perspective of rogue seasonality. There are very few empirical supply chain studies based on multi echelon structures so this study contributes to the same while also providing insights about the causes and characteristic presentation of rogue seasonality from a practical perspective.

Rogue seasonality management by the sense and respond approach: Only a few studies in supply chain management have explored the application of the sense and respond approach, despite its utility (for managing disturbances) being highlighted by many researchers and it being successfully applied in various other disciplines. This study does so for the rogue seasonality context. All the decision elements pertaining to the application of the sense and respond approach are determined in the thesis which include the signature and index for indicating the presence/absence and intensity of rogue seasonality, the system variables and the time series transformation to be used (among various alternatives) and the process based on using this information to manage rogue seasonality. A comprehensive analysis was used for determination of these decision elements unlike recent related studies such as by Thornhill and Naim (2006) whose findings on identification of rogue seasonality are based on a single case study, a single technique for detection and a subjective detection process.

The thesis has also contributed in respect of the decision elements as follows.

Measure of rogue seasonality: Unlike the Bullwhip effect for which multiple measures have been proposed, there is no measure for rogue seasonality. This thesis has proposed an index, which indicates the intensity of rogue seasonality in a multi echelon structure and could be used to identify problematic supply chains in practical settings. A comparison of the normalized profiles of variables is associated with the index computation i.e. the amplification in the variables is eliminated, which means that the index gives an assessment of rogue seasonality in a supply chain system which is independent of Bullwhip.

Time series transformation techniques (and features) evaluated: Time series clustering studies in the literature (across domains) have generally evaluated one/few transformation techniques (and features) and used non contextual simulated data or data 
on stock prices.for the same. The findings from these studies are therefore, less valid for the supply chain context as the appropriate features for time series clustering are domain specific. All the techniques (and features) for time series clustering which were assessed in previous studies individually, were used in the evaluation and include time, Fourier (FT), discrete wavelet (DWT), autocorrelation function (ACF), cross correlation function (CCF) and autoregressive model (AR model). Also, supply chain contextual data, both simulated and empirical, was used in the evaluation. The contribution of the thesis is therefore in establishing the best technique (and feature) for clustering time series data from the supply chain domain including for rogue seasonality. FT feature was identified as that best technique, which, though similar to the finding in Thornhill and Naim (2006), was determined on the basis of a rigourous evaluation process involving a greater number of alternative techniques and different rogue seasonality contexts.

The thesis has also made a minor contribution in terms of the Excel-VBA-Matlab process used for carrying out the large scale simulation which the author has not come across. This process was used in the simulation analysis of both linear as well as non-linear systems. Another minor contribution relates to the frequency response based analysis of the Beer game system in the thesis which has not been considered in the past.

\subsection{Limitations of the research and suggestions for further study}

Though this thesis carried out a comprehensive investigation on rogue seasonality generation and its detection, it still has a few limitations which are summarised below.

a) Supply networks were not considered in the analysis except for the grocery case study, with most of the analysis being based on supply chains. Though networks can be treated as combinations of supply chains, the dynamics could change in case of interference between parallel chains. An important question is whether the signature and index would be valid for network structures and, if not, what modifications are required for various network configurations.

b) The index based identification of the problematic supply chain is useful. However, it would be more useful if the primary culprit (echelon) responsible for the high rogue 
seasonality intensity is also identified at the same time. Computing the index values at select points along the supply chain/network could probably be used for the same.

c) This study proposed a flowchart for managing rogue seasonality on the basis of detecting it using the index. This flowchart needs to be tested and validated in an empirical setting with the same applying to the index as well.

d) Finally in terms of techniques, though all the contemporary techniques and which could be easily interpreted from a management perspective were tried, use of new techniques such as Support Vector Machines could be explored in order to improve the detection performance especially in more complex systems. 


\section{REFERENCES}

Agrawal, R., Faloutsos, C., and Swami, A. (1993). "Efficient similarity search in sequence databases." Proc. of the 4th International conference on foundations of data organization and algorithms, Chicago, IL, USA, 69-84.

AIRMIC, A., IRM (2002). "A Risk Management Standard."

Akkermans, H., and Dellaert, N. (2005). "The rediscovery of industrial dynamics: the contribution of system dynamics to supply chain management in a dynamic and fragmented world." System Dynamics Review, 21(3), pp. 173-269.

Anderson Jr, E. G., Morrice, D. J., and Lundeen, G. (2005). "The "physics" of capacity and backlog management in service and custom manufacturing supply chains." System Dynamics Review, 21(3), pp. 217-247.

Angerhofer, B. J., and Angelides, M. C. (2000). "System dynamics modelling in supply chain management: research review." Proceedings of the Winter simulation conference, Orlando, Florida, 342-351.

Ansoff, H. I., and Slevin, D. P. (1968). "An appreciation of industrial dynamics." Management Science, 14(7), pp. 383-397.

Aono, M., Sekiguchi, Y., Yasuda, Y., Suzuki, N., and Seki, Y. (2006). "Time Series Data Mining for Multimodal Bio-Signal Data." IJCSNS International Journal of Computer Science and Network Security, 6(10), pp. 1-9.

Arbnor, I., and Bjerke, B. (1997). Methodology for Creating Business Knowledge, Sage Publications, Newbury Park, CA

Arlbjørn, J. S., and Halldorsson, A. (2002). "Logistics knowledge creation: reflections on content, context and processes." International Journal of Physical Distribution \& Logistics Management, 32(1), pp. 22-40.

Axater, S. (1985). "Control theory concepts in production and inventory control." International Journal of Systems Science, 16(2), pp. 161-169.

Axater, S. (2000). Inventory Control, Kluwer Academic Publishers, Boston.

Baader, A., and Montanus, S. (2008). "Transparency in global supply chain networks." Strategies and Tactics in Supply Chain Event Management, R. Ijioui, H. Emmerich, and M. Ceyp, eds., Springer-Verlag Berlin, 3-11.

Banks, J., Carson, J. S., and Nelson, B. L. (1999). Discrete-Event System Simulation, Prentice Hall, New Jersey.

Baragona, R. (2001). "A simulation study on clustering time series with metaheuristic methods." Quaderni di Statistica, 3.

Barrett, L. (2001). "'Cisco's \$2.25 Billion Mea Culpa", CNETnews.com, May 9, 2001."

Basseville, M., and Nikiforov, I. V. (1993). Detection of Abrupt Changes: Theory and Application, Prentice Hall, Englewood Cliffs.

Batson, R. G., and McGough, K. D. (2007). "A new direction in quality engineering: Supply chain quality modelling." International Journal of Production Research, 45(23), pp. 5455-5464.

Bendoly, E., and Cotteleer, M. J. (2008). "Understanding behavioral sources of process variation following enterprise system deployment." Journal of Operations Management, 26(1), pp. 23-44.

Bendoly, E., Donohue, K., and Schultz, K. L. (2006). "Behavior in operations management: Assessing recent findings and revisiting old assumptions." Journal of Operations Management, 24(6), pp. 737-752. 
Bendoly, E., and Speier, C. (2008). "Silver bullet junkies and the codifiers that love them: Behavioral roots behind a legacy of bad modeling and use." Decision Sciences, 39(2), pp. 157-173.

Berry, D., Naim, M. M., and Towill, D. R. (1995). "Business process re-engineering an electronic products supply chain." IEE Proceedings-Science, Measurement and Technology: Special Issue on Manufacturing 142(5), pp. 395-403.

Bissell, C. C. (1996). Control Engineering, Chapman \& Hall, London.

Blackhurst, J., Craighead, C. W., Elkins, D., and Handfield, R. B. (2005). "An empirically derived agenda of critical research issues for managing supply chain disruptions." International Journal of Production Research, 43(19), pp. 40674081 .

Blackhurst, J. V., Scheibe, K. P., and Johnson, D. J. (2008). "Supplier risk assessment and monitoring for the automotive industry." International Journal of Physical Distribution \& Logistics Management, 38(2), pp. 143-165.

Bodendorf, F., and Zimmermann, R. (2005). "Proactive Supply-Chain Event Management with Agent Technology." International Journal of Electronic Commerce, 9(4), pp. 57-89.

Bohte, Z., Cepar, D., and Kosmelj, K. (1980). "Clustering of time series." Proceedings in Computational Statistics, Physica-Verlag.

Box, G. E. P., and Jenkins, G. M. (1976). Time series analysis : forecasting and control, Holden-Day, San Francisco.

Boyer, K. K., and Swink, M. L. (2008). "Empirical Elephants-Why Multiple Methods are Essential to Quality Research in Operations and Supply Chain Management " Journal of Operations Management, 26(3), pp. 338-344.

Bryman, A., and Bell, E. (2004). Business Research Methods Oxford University Press, USA

Buckeridge, D. L., Burkom, H., Campbell, M., Hogan, W. R., and Moore, A. W. (2005). "Algorithms for rapid outbreak detection: A research synthesis." Journal of Biomedical Informatics, 38(2), pp. 99-113.

Buckley, S., Ettl, M., Lin, G., and Wang, K.-Y. (2005). "Sense and Respond Business Performance Management." Supply Chain Management on Demand: Strategies and Technologies, Applications, C. An and H. Fromm, eds., Springer, New York, 287-311.

Burbidge, J. L. (1989). Production flow analysis, Oxford University Press, Oxford, UK.

Buxey, G. (1995). "A managerial perspective on aggregate planning." International Journal of Production Economics, 41(1-3), pp. 127-133.

Cachon, G., and Lariviere, M. A. (1999a). "Capacity allocation with past sales: when to turn-and-earn." Management Science 45(5), pp. 685-703.

Cachon, G., and Lariviere, M. A. (1999b). "Capacity choice and allocation: strategic behavior and supply chain performance." Management Science 45(8), pp. 10911108.

Cachon, G., and Lariviere, M. A. (1999c). "An equilibrium analysis of linear and proportional allocation of scarce capacity." IIE Transactions, 31(9), pp. 835-850.

Cachon, G. P. (1999). "Managing Supply Chain Demand Variability with Scheduled Ordering Policies." Management Science, 45(6), pp. 843-856.

Caiado, J., Crato, N., and Pena, D. (2006). "A periodogram-based metric for time series classification." Computational Statistics and Data Analysis, 50(10), pp. 2668-2684. 
Cavinato, J. L. (2004). "Supply chain logistics risks From the back room to the board room." International Journal of Physical Distribution \& Logistics Management, 34(5), pp. 383-387.

Chan, K. P., and Fu, A. W. C. (1999). "Efficient Time Series Matching by Wavelets." Proceedings Of The International Conference On Data Engineering, Sydney, Australia, 126-135.

Chatfield, C. (2004). The Analysis of Time Series: An Introduction, CRC Press, Boca Raton, Florida.

Chen, F., Drezner, Z., Ryan, J. K., and Simchi-Levi, D. (2000). "Quantifying the bullwhip effect in a simple supply chain: the impact of forecasting, lead times, and information." Management Science, 46(3), pp. 436-443.

Chopra, S., and Meindl, P. (2004). Supply chain management : strategy, planning, and operation, Prentice Hall, Upper Saddle River, N.J.

Chopra, S., and Sodhi, M. S. (2004). "Managing risk to avoid supply-chain breakdown." MIT Sloan Management Review, 46(1).

Christopher, M. (1992). Logistics and Supply Chain Management : Strategies for Reducing Costs and Improving Service, Pitman.

Christopher, M., and Lee, H. (2004). "Mitigating supply chain risk through improved confidence." International Journal of Physical Distribution \& Logistics Management, 34(5), pp. 388-396.

Christopher, M. G. (2002). "Supply Chain Vulnerability, report for Department of Transport,Local Government and the Regions,." Cranfield University, Cranfield.

Cortes, C., and Pregibon, D. (2001). "Signature-based methods for data streams." Data Mining and Knowledge Discovery, 5(3), pp. 167-182.

Coyle, R. G. (1977). Management System Dynamics, John Wiley \& Sons, New York.

Craighead, C. W., Blackhurst, J., Rungtusanatham, M. J., and Handfield, R. B. (2007). "The severity of supply chain disruptions: Design characteristics and mitigation capabilities." Decision Sciences, 38(1), pp. 131-156.

Croson, R., and Donohue, K. (2003). "Impact of POS data sharing on supply chain management: An experimental study." Production and Operations Management, 12(1), pp. 1-11.

Croson, R., and Donohue, K. (2006). "Behavioral Causes of the Bullwhip Effect and the Observed Value of Inventory Information." Management Science, 52(3), pp. 323-336.

Croson, R., Donohue, K., Katok, E., and Sterman, J. (2005). "Order Stability in Supply Chains:Coordination Risk and the Role of Coordination Stock." Working Paper, Penn State University.

Cruz, A. M. L. D. L., Veeke, H. P. M., and Lodewijks, G. (2007). "Intelligent supply chain by using prognostic logistics." International Journal of Service Operations and Informatics, 2(2), pp. 152-163.

Daganzo, C. F. (2004). "On the stability of supply chains." Operations Research, 52(6), pp. 909-921.

Davis, T. (1993). "Effective Supply Chain Management." Sloan Management Review, 34(4), pp. 35-46.

De Souza, R., Zice, S., and Chaoyang, L. (2000). "Supply chain dynamics and optimization." Integrated Manufacturing Systems, 11(4), pp. 348-364.

Dejonckheere, J., Disney, S., Lambrecht, M., and Towill, D. R. (2003). "Measuring and avoiding the bullwhip effect: A control theoretic approach." European Journal of Operational Research 147, pp. 567-590. 
Dejonckheere, J., Disney, S., Lambrecht, M., and Towill, D. R. (2004). "The impact of information enrichment on the Bullwhip effect in supply chains: A control engineering perspective." European Journal of Operational Research 153, pp. 727-750.

Deng, K., Moore, A. W., and Nechyba, M. C. "Learning to recognize time series: Combining ARMA models with memory-based learning." Proceedings of IEEE International Symposium on Computational Intelligence in Robotics and Automation, CIRA, 246-251.

Denzin, N. K., and Lincoln, Y. S. (1994). Handbook of Qualitative Research, Sage Publications, Thousand Oaks, CA.

Disney, S. M., and Lambrecht, M. R. (2007). "On replenishment rules, forecasting and the Bullwhip effect in supply chains." Foundations and Trends ${ }^{\circledR}$ in Technology, Information and Operations Management, 2(1), pp. 1-80.

Disney, S. M., Naim, M. M., and Potter, A. (2004). "Assessing the impact of ebusiness on supply chain dynamics " International Journal of Production Economics, 89(2), pp. 109-118.

Disney, S. M., and Towill, D. R. (2002). "A Discrete Transfer Function Model to Determine the Dynamic Stability of a Vendor Managed Inventory Supply Chain." International Journal of Production Research, 40(1), pp. 179-204.

Disney, S. M., and Towill, D. R. (2003a). "The effect of vendor managed inventory (VMI) dynamics on the Bullwhip Effect in supply chains." International Journal of Production Economics, 85(2), pp. 199-215.

Disney, S. M., and Towill, D. R. (2003b). "On the bullwhip and inventory variance produced by an ordering policy." OMEGA: The International Journal of Management Science, 31(3), pp. 157-167.

Disney, S. M., and Towill, D. R. (2005). "Eliminating drift in inventory and order based production control systems "International Journal of Production Economics, 93-94, pp. 331-344.

Disney, S. M., and Towill, D. R. (2006). "A methodology for benchmarking replenishment-induced bullwhip." Supply Chain Management - An International Journal, 11(2), pp. 160-168.

Disney, S. M., Towill, D. R., and Warburton, R. D. H. (2006). "On the equivalence of control theoretic, differential, and difference equation approaches to modelling supply chains " International Journal of Production Economics, 101(1), pp. 194208.

Donoho, S. (2004). " Early Detection of Insider Trading in Option Markets." Tenth International Conference on Knowledge Discovery and Data Mining, Seattle, Washington, USA.

Dzwinel, W., Yuen, D. A., Boryczko, K., Ben-Zion, Y., Yoshioka, S., and Ito, T. (2003). "Cluster Analysis, Data-Mining, Multi-dimensional Visualization of Earthquakes over Space, Time and Feature Space." Submitted to Earth and Planetary Sci. Letters.

Edge, M. E., and Falcone Sampaio, P. R. (2009). "A survey of signature based methods for financial fraud detection." Computers and Security.

Edghill, J. S., and Towill, D. R. (1989). "The use of systems dynamics in manufacturing systems." Transactions of the Institute of Measurement \& Control, $11(4)$, pp. 208-216.

Eisenhardt, K. M. (1989). "Building theories from case study research." Academy of Management Review 14(4), pp. 532-550. 
Elkins, D., Handfield, R. B., Blackhurst, J., and Craighead, C. W. (2008). "A "To-Do" list to improve supply chain risk management capabilities " Supply Chain Risk Management - Minimising Disruptions in Global Sourcing, R. B. Handfield and K. McCormack, eds., Auerbach Publications London, 57-63.

Evans, G., Cheema, P. and Towill, D. (1997). "Analysis and design of an adaptive, minimum inventory control system." Production Planning and Control, 8(6), pp. 545-557.

Everitt, B. S. (1993). Cluster analysis, Arnold Publishing, London.

Fawcett, T., and Provost, F. (1997). "Adaptive fraud detection." Data Mining and Knowledge Discovery, 1(3), pp. 291-316.

Fliedner, G. (2003). "CPFR: An emerging supply chain tool." Industrial Management and Data Systems 103(1/2), pp. 14-21.

Forrester, J. W. (1961). Industrial dynamics, M.I.T. Press, Cambridge, Mass.

Fowler, A. (1999). "Feedback and feedforward as systemic frameworks for operations control." International Journal of Operations and Production Management, 19(2), pp. 182-204.

Frankel, R., Naslund, D., and Bolumole, Y. (2005). "Entering the white space of logistics research." Journal of Business Logistics, 26(2), pp. 185-209.

Fransoo, J. C., and Wouters, M. J. (2000). "Measuring the bullwhip effect in the supply chain." Supply Chain Management: An International Journal 5(2), pp. 7889.

Ganeshan, R., and Harrison, T. (1995). "An Introduction to Supply Chain Management." Department of Management Science and Information Systems, Pennsylvania State University, Philadelphia.

Gaonkar, R., and Viswanadham, N. (2004). "A Conceptual and Analytical Framework for the Management of Risk in Supply Chains." Indian School of Business working paper, 1-20.

Geary, S., Childerhouse, P., and Towill, D. R. (2002). "Uncertainty and the seamless supply chain." Supply Chain Management Review, 6(4), pp. 52-61.

Geary, S., Disney, S. M., and Towill, D. R. (2006). "On bullwhip in supply chains-historical review, present practice and expected future impact." International Journal of Production Economics, 101(1), pp. 2-18.

Gilbert, K. (2005). "An ARIMA supply chain model." Management Science, 51(2), pp. 305-310.

Giunipero, L. C., and Eltantawy, R. A. (2004). "Securing the upstream supply chain: a risk management approach." International Journal of Physical Distribution \& Logistics Management, 34(9), pp. 698-713.

Goutte, C., Toft, P., Rostrup, E., Nielsen, F. A., and Hansen, L. K. (1999). "On clustering fMRI time series." Neurolmage, 9(3), pp. 298-310.

Gottman, J. M. (1981). Time-Series Analysis: A Comprehensive Introduction for Social Scientists, Cambridge University Press, Cambridge

Greasley, A. (2004). Simulation Modelling for Business, Ashgate. Publishing Ltd, Aldershot.

Guba, E. G. (1990). The Paradigm Dialog, Sage Publications, Newbury Park, CA

Haeckel, S. H. (1999). Adaptive Enterprise: Creating and Leading Sense-AndRespond Organizations HBS Press, Boston.

Haeckel, S. H., and Nolan, R. L. (1993). "Managing by wire." Harvard Business Review, 71(5), pp. 122-132.

Halkidi, M., Batistakis, Y., and Vazirgiannis, M. (2001). "On clustering validation techniques." Journal of Intelligent Information Systems, 17(2-3), pp. 107-145. 
Hallikas, J., Virolainen, V.-M., and Tuominen, M. (2002). "Risk analysis and assessment in network environments: A dyadic case study." International Journal of Production Economics, 78(1), pp. 45-55.

Han, J., and Kamber, M. (2006). Data Mining:Concepts and Techniques, Elsevier.

Handfield, R. B., Blackhurst, J., Elkins, D., and Craighead, C. W. (2008). "A framework for reducing the impact of disruptions to the supply chain: Observations from Multiple Executives." Supply Chain Risk Management Minimising Disruptions in Global Sourcing, R. B. Handfield and K. McCormack, eds., Auerbach Publications London, 29-49.

Harland, C., Brenchley, R., and Walker, H. (2003). "Risk in supply networks." Journal of Purchasing and Supply Management, 9, pp. 51-62.

Hausman, W. H. (2003). "Supply chain performance metrics." The Practice of Supply Chain Managementm : Where theory and application converge, C. Billington, T. Harrison, H. Lee, and J. Neale, eds., Kluwer, Boston.

Haywood, M., and Peck, H. (2004). "Supply chain vulnerability within UK aerospace manufacturing: Development of a vulnerability management toolkit." Supply Chain Practice, 6(1), pp. 72-83.

Hejazi, A., and Hilmola, O.-P. (2006). "Manufacturing lot size as a source of the Bullwhip effect: a case study of electronics and furniture supply chains." International Journal of Services and Operations Management, 2(3), pp. 237-255.

Helo, P.T. (2000), "Dynamic modelling of surge effect and capacity limitation in supply chains", International Journal of Production Research, 38(17), pp. 45214533.

Hendricks, K. B., and Singhal, V. R. (2003). "The effect of supply chain glitches on shareholder wealth " Journal of Operations Management, 21(5), pp. 501-522.

Hendricks, K. B., and Singhal, V. R. (2005). "Association between supply chain glitches and operating performance." Management Science, 51(5), pp. 695-711.

Hoek, R. v. (2002). "Using information technology to leverage transport and logistics service operations in the supply chain: an empirical assessment of the interrelation between technology and operations management." International Journal of Information Technology and Management, 1(1), pp. 115-130.

Holland, W., and Sodhi, M. S. (2004). "Quantifying the Effect of Batch Size and Order Errors on the Bullwhip Effect Using Simulation." International Journal of Logistics: Research and Applications, 7(3), pp. 251-261.

Hong-Minh, S. M., Disney, S. M., and Naim, M. M. (2000). "The dynamics of emergency transhipment supply chains." International Journal of Physical Distribution and Logistics Management 30(9), pp. 788-815.

Houlihan, J. B. (1987). "International supply chain management." International Journal of Physical Distribution and Materials Management 17(2), pp. 51-66.

Hwarng, H. B., and Xie, N. (2008). "Understanding supply chain dynamics: A chaos perspective." European Journal of Operational Research, 184(3), pp. 1163-1178.

Jaksic, M., and Rusjan, B. (2008). "The effect of replenishment policies on the bullwhip effect: A transfer function approach." European Journal Of Operational Research, 184(3), pp. 946-961.

Jeong, M. K., Lu, J. C., Huo, X., Vidakovic, B., and Chen, D. (2006). "Wavelet-based data reduction techniques for process fault detection." Technometrics, 48(1), pp. 26-40.

Jick, T. D. (1979). "Mixing qualitative and quantitative methods: triangulation in action." Administrative Science Quarterly 24(4), pp. 602-611. 
John, S., Naim, M. M., and Towill, D. R. (1994). "Dynamic analysis of a WIP compensated decision support system." International Journal of Manufacturing System Design, 1(4), pp. 283-297.

Johnson, M. E. (2001). "Learning from toys: Lessons in managing supply chain risk from the toy industry." California Management Review, 43(3), pp. 106-124.

Juttner, U. (2005). "Supply Chain risk management - Understanding the business requirements from a practitioner perspective." International Journal of Logistics Management, 16(1), pp. 120-141.

Juttner, U., Peck, H., and Christopher, M. (2003). "Supply Chain Risk Management: Outlining an Agenda for Future Research." International Journal of Logistics: Research and Applications, 6(4), pp. 197-210.

Kahveci, T., and Singh, A. "Variable length queries for time series data." 17th International Conference on Data Engineering, Heidelberg, Germany, 273-282.

Kaipia, R., Korhonen, H., and Hartiala, H. (2006). "Planning nervousness in a demand supply network: an empirical study." International Journal of Logistics Management, 17(1), pp. 95-113.

Kalpakis, K., Gada, D., and Puttagunta, V. (2001). "Distance Measures for Effective Clustering of ARIMA Time-Series." Proceedings of the 2001 IEEE International Conference on Data Mining, IEEE Computer Society, San Jose, CA.

Karkkainen, M., Ala-Risku, T., and Framling, K. (2004). "Efficient tracking for shortterm multi-company networks." International Journal of Physical Distribution \& Logistics Management, 34(7), pp. 545-564.

Kelle, P., and Milne, A. (1999). "The effect of $(\mathrm{s}, \mathrm{S})$ ordering policy on the supply chain." International Journal of Production Economics, 59, pp. 113-122.

Keogh, E., and Kasetty, S. (2003). "On the Need for Time Series Data Mining Benchmarks A Survey and Empirical Demonstration." Data Mining And Knowledge Discovery, 7(4), pp. 349-371.

Kim, I., and Springer, M. (2008). "Measuring endogenous supply chain volatility: Beyond the bullwhip effect." European journal of operational research., 189(1), pp. 172-193.

Kleijnen, J. P. C. (2005). "Supply chain simulation tools and techniques: a survey." International Journal of Simulation \& Process Modelling, 1(1/2), pp. 82-89.

Kleindorfer, P. R., and Saad, G. H. (2005). "Managing disruption risks in supply chains." Production and Operations Management, 14(1), pp. 53-68.

Lamma, E., Mello, P., Nanetti, A., Riguzzi, F., Storari, S., and Valastro, G. (2006). "Artificial intelligence techniques for monitoring dangerous infections." IEEE Transactions on Information Technology in Biomedicine, 10(1), pp. 143-155.

Lane, D. C. (2000). "You just don't understand me: Modes of failure and success in the discourse between system dynamics and discrete event simulation." LSE OR Dept Working Paper LSEOR 00-34, London School of Economics and Political Science, London.

Larsen, E. R., Morecroft, J. D. W., and Thomsen, J. S. (1999). "Complex behaviour in a production-distribution model." European Journal of Operational Research, 119, pp. 61-74.

Law, A. M., and Kelton, W. D. (2000). Simulation Modelling and Analysis, McGrawHill, New York.

Lawrie, G. (2003). "Preparing for adaptive supply networks, TechStrategy Report." Forrester Inc. 
LeBlanc, L. J., and Grossman, T. A. (2008). "The Use of Spreadsheet Software in the Application of Management Science and Operations Research." Interfaces, 38(4), pp. 225-227.

Lee, H., and Billington, C. (1993). "Material management in decentralized supply chains." Operations Research, 41(5), pp. 835-847.

Lee, H. L., Padmanabhan, V., and Whang, S. (1997a). "The Bullwhip effect in supply chains." Sloan Management Review, Spring, pp. 93-102.

Lee, H. L., Padmanabhan, V., and Whang, S. (1997b). "Information distortion in a supply chain: The bullwhip effect." Management Science 43(4), pp. 546-558.

Lee, H. L., and Whang, S. (2000). "Information sharing in a supply chain." International Journal of Technology Management 20(3/4), pp. 373-387.

Lee, J., Ni, J., Djurdjanovic, D., Qiu, H., and Liao, H. (2006). "Intelligent prognostics tools and e-maintenance." Computers in Industry, 57(6), pp. 476-489.

Lee, W., and Stolfo, S. J. (1998). "Data mining approaches for intrusion detection." Seventh USENIX Security Symposium, San Antonio, Texas.

Li, T., Li, Q., Zhu, S., and Ogihara, M. (2002). "A survey on wavelet applications in data mining." SIGKDD Explor. Newsl., 4(2), pp. 49-68.

Liao, T. W. (2005). "Clustering of time series data-a survey." Pattern recognition., 38(11), pp. 1857-1874.

Lin, J., Vlachos, M., Keogh, E., and Gunopulos, D. (2004). "Iterative Incremental Clustering of Time Series." Advances in Database Technology - EDBT 2004, E. Bertino, ed., Springer- Verlag, Berlin / Heidelberg, 106-122.

Lindroth, R., and Norrman, A. "Supply Chain Risks and Risk Sharing Instruments An Illustration from the Telecommunication Industry." Logistics Research Network 6th Annual Conference, Edinburgh, 297-307.

Loch, C. H., and Wu, Y. (2007). "Behavioural Operations Management." Foundations and Trends ${ }^{\circledR}$ in Technology, Information and Operations Management, 1(3), pp. 121-232.

Lummus, R. R., Duclos, L. K., and Vokurka, R. J. (2003). "The impact of marketing initiatives on the supply chain." Supply Chain Management - An International Journal, 8(4), pp. 317-323.

Maharaj, E. A. (2000). "Clusters of time series." Journal of Classification, 17(2), pp. 297-314.

Mason-Jones, R., Naim, M. M., and Towill, D. R. (1997). "The impact of pipeline control on supply chain dynamics." International Journal of Logistics Management, 8(2), pp. 47-62.

Mason-Jones, R., and Towill, D. R. (1998). "Shrinking the Supply Chain Uncertainty Circle." Institute of Operations Management Control Journal, 24(7), pp. 17-23.

Mathiassen, L., and Vainio, A. M. (2007). "Dynamic Capabilities in Small Software Firms:A Sense-and-Respond Approach." IEEE Transactions on Engineering Management, 54(3), pp. 522-538.

McCarthy, B. L., and Wasusri, T. (2002). "A review of non-standard applications of statistical process control (SPC) charts." International Journal of Quality and Reliability Management, 19(3), pp. 295-320.

Mccarthy, I. P., and Tan, Y. K. (2000). "Manufacturing competitiveness and fitness landscape theory." Journal of Materials Processing Technology, 107(1-3), pp. 347352.

McCullen, P., and Towill, D. R. (2002). "Diagnosis and reduction of bullwip in supply chains." Supply Chain Management: An International Journal, 7(3), pp. 164-180. 
McHutchon, M. A., Staszewski, W. J., and Schmid, F. (2005). "Signal processing for remote condition monitoring of railway points." Strain, 41(2), pp. 71-85.

Menotti, M. (August 2004). "The Sense-and-Respond Enterprise: Why the U.S. Marine Corps Should Embrace the New Paradigm." OR/MS Today.

Mentzer, J. T., and Kahn, K. B. (1995). "A Framework for Logistics Research." Journal of Business Logistics, 16(1), pp. 231-251.

Metters, R. (1997). "Quantifying the Bullwhip Effect in Supply Chains." Journal of Operations Management, 15(2), pp. 89-100.

Miles, M. B., and Huberman, A. M. (1994). Qualitative data analysis Sage Publications, Thousand Oaks, CA.

Miragliotta, G. (2006). "Layers and mechanisms: A new taxonomy for the Bullwhip Effect." International Journal Of Production Economics, 104(2), pp. 365-381.

Montgomery, D. C. (2005). Introduction to statistical quality control John Wiley \& Sons, New Jersey.

Morecroft, J., and Robinson, S. "Explaining Puzzling Dynamics: Comparing the Use of System Dynamics and Discrete-Event Simulation." The 23rd International Conference of the System Dynamics Society, Boston.

Naim, M. M., and Towill, D. R. (1994). "Establishing a Framework for effective Materials Logistics Management." International Journal of Logistics Management 5(1), pp. 81-88.

Naslund, D. (2002). "Logistics needs qualitative research - especially action research." International Journal of Physical Distribution \& Logistics Management, 32(5), pp. 321-338.

Nise, N. S. (1995). Control Systems Engineering, Benjamin/Cummings, California.

Norrman, A., and Jansson, U. (2004). "Ericsson's proactive supply chain risk management approach after a serious sub-supplier accident." International Journal of Physical Distribution \& Logistics Management, 34(5), pp. 434-456.

O'Donnell, T., Maguire, L., McIvor, R., and Humphreys, P. (2006). "Minimizing the bullwhip effect in a supply chain using genetic algorithms." International Journal of Production Research, 44(8), pp. 1523-1543.

Olavson, T., and Fry, C. (2008). "Spreadsheet Decision-Support Tools: Lessons Learned at Hewlett-Packard." Interfaces, 38(4), pp. 300-310.

Olsson, E., Funk, P., and Xiong, N. (2004). "Fault diagnosis in industry using sensor readings and case-based reasoning." Journal of Intelligent and Fuzzy Systems, 15(1), pp. 41-46.

Ortega, M., and Lin, L. (2004). "Control theory applications to the productioninventory problem: a review." International Journal of Production Research, 42(11), pp. 2303-2322.

Otto, A. (2003). "Supply Chain Event Management: Three Perspectives " International Journal of Logistics Management, 14(2), pp. 1-13.

Paik, S. K., and Bagchi, P. K. (2007). "Understanding the causes of the bullwhip effect in a supply chain." International Journal of Retail and Distribution Management, 35(4), pp. 308-324.

Parunak, H. V. D. (1999). "Industrial and practical applications of DAI." Multiagent systems: a modern approach to distributed artificial intelligence, MIT Press, pp. 377-421.

Percival, D. B., and Walden, A. T. (2000). Wavelet methods for time series analysis, Cambridge University Press, Cambridge; New York.

Pettigrew, A. (1990). "Longitudinal field research on change: theory and practice." Organisation Science, 1(3), pp. 267-292. 
Phua, C., Lee, V., Smith, K., and Gayler, R. (2005). "A comprehensive survey of data mining-based fraud detection research."

Piccolo, D. (1990). "A distance measure for classifying ARIMA models." Journal of time series analysis, 11(2).

Poirier, C., and Quinn, F. (2003). "Calibration supply chain management." Computer Sciences Corporation Report.

Popplewell, K., and Bonney, M. C. (1987). "Application of Discrete Linear Control Theory of the Analysis and Simulation of Multi-Product, Multi-Level Production Control Systems." International Journal of Production Research, 25(1), pp. 45-56.

Potter, A., and Disney, S. M. (2006). "Bullwhip and batching: an exploration." International Journal of Production Economics, 104(2), pp. 408-418.

Potter, A., and Lalwani, C. (2008). "Investigating the impact of demand amplification on freight transport " Transportation Research Part E: Logistics and Transportation Review, 44(5), pp. 835-846.

Radjou, N. (2002). "Executive overview: Adaptive supply networks, Techstrategy Report." Forrester Inc.

Raheja, D., Llinas, J., Nagi, R., and Romanowski, C. (2006). "Data fusion/data mining-based architecture for condition-based maintenance." International Journal of Production Research, 44(14), pp. 2869-2887.

Rapoport, A. (1966). "A study of human control in a stochastic multistage decision task." Behavioral Sciences, 11, pp. 18-32.

Rapoport, A. (1967). "Variables affecting decisions in a multistage inventory task." Behavioral Sciences, 12, pp. 192-204.

Riddalls, C. E., and Bennett, S. (2001). "The optimal control of batched production and its effect on demand amplification." International Journal of Production Economics, 72, pp. 159-168.

Riddalls, C. E., Bennett, S., and Tipi, N. S. (2000). "Modelling the dynamics of supply chains." International Journal of Systems Science, 31(8), pp. 969-976.

Sachan, A., and Datta, S. (2005). "Review of supply chain management and logistics research." International Journal of Physical Distribution \& Logistics Management, 35(9), pp. 664-705.

Safizadeh, M. H. and Ritzman, L. P. (1997). "Linking performance drivers in production planning and inventory control to process choice." Journal of Operations Management, 15(4), pp. 389-403.

Sarimveisa, H., Patrinosa, P., Tarantilis, C. D., and Kiranoudis, C. T. (2008). "Dynamic modeling and control of supply chain systems: A review." Computers and Operations Research, 35, pp. 3530-3561.

Saunders, M. N. K., Lewis, P., and Thornhill, A. (2002). Research Methods for Business Students Prentice Hall, Essex, UK.

Schweitzer, M. E., and Cachon, G. P. (2000). "Decision bias in the newsvendor problem with a known demand distribution: experimental evidence." Management Science, 46(3), pp. 404-420.

Seaker, R. F., Waller, M. A., and Dunn, S. C. (1993). "A note on research methodology in business logistics." Logistics and Transportation Review, 29(4), pp. 383-387.

Shannon, R. E. (1975). Systems Simulation: The Art and Science Prentice Hall, New Jersey.

Sheffi, Y. (2001). "Supply chain management under the threat of international terrorism." International Journal of Logistics Management, 12(2), pp. 1-11. 
Shukla, V., and Naim, M. M. (2009). "The Impact of Capacity Constraints on Supply Chain Dynamics " 39th International Conference on Computers \& Industrial Engineering Troyes, France.

Shukla, V., Naim, M. M., and Yaseen, E. A. (2009). "Bullwhip and backlash in supply pipelines." International Journal of Production Research, 47(23), pp. 64776497.

Simchi-Levi, D., Kaminsky, P., and Simchi-Levi, E. (2002). Designing and Managing the Supply Chain, McGraw-Hill, Singapore.

Simon, H. A. (1952). "On the application of servomechanism theory in the study of production control." Econometrica, 20(2), pp. 247-268.

Smith, G. A. (2003). "Using integrated spreadsheet modeling for supply chain analysis." Supply Chain Management: An International Journal 8(4), pp. 285-290.

Stauffer, D. (2003). "Supply Chain Risk: Deal with it." Harvard Business School.

Sterman, J. (1989). "Modelling managerial behaviour: Misperceptions of feedback in a dynamic decision-making experiment." Management Science 35(3), pp. 321339.

Sterman, J. D. (2000). Business dynamics: systems thinking and modelling for a complex world, McGraw-Hill, Boston.

Stevens, G. (1989). "Integrating the supply chain." International Journal of Physical Distribution and Materials Management, 19(8), pp. 3-8.

Stock, J. R. (1997). "Applying theories from other disciplines to logistics." International Journal of Physical Distribution and Logistics Management, 27(9/10), pp. 515-539.

Stuart, I., McCutcheon, D., Handfield, R., McLachlin, R., and Samson, D. (2002). "Effective case research in operations management: a process perspective." Journal of Operations Management, 20(4), pp. 419-433.

$\mathrm{Su}, \mathrm{X}$. (2008). "Bounded rationality in newsvendor models." Manufacturing and Service Operations Management, 10(4), pp. 566-589.

Svensson, G. (2000). "A Conceptual framework for the analysis of vulnerability in supply chains." International Journal of Physical Distribution \& Logistics Management, 30(9), pp. 731-749.

Sweetser, A. "A Comparison of System Dynamics and Discrete Event Simulation." Proceedings of 17th International Conference of the System Dynamics Society and 5th Australian \& New Zealand Systems Conference., Wellington, New Zealand.

Tako, A. A., and Robinson, S. (2009). "Comparing discrete-event simulation and system dynamics: users' perceptions." Journal of the Operational Research Society 60, pp. 296-312.

Tan, P.-N., Steinbach, M., and Kumar, V. (2005). Introduction to Data Mining, Addison-Wesley Longman, Boston.

Tang, C., and Tomlin, B. (2008). "The power of flexibility for mitigating supply chain risks." International Journal of Production Economics, 116, pp. 12-27.

Tang, C. S. (2006). "Perspectives in supply chain risk management " International Journal of Production Economics, 103, pp. 451-488.

Tang, O., and Naim, M. M. (2004). "The impact of information transparency on the dynamic behaviour of a hybrid manufacturing/remanufacturing system." International Journal of Production Research, 42(19), pp. 4135-4152.

Taylor, D. H. (1999). "Measurement and analysis of demand amplification across the supply chain." International Journal of Logistics Management, 10(2), pp. 55-70.

Terzi, S., and Cavalieri, S. (2004). "Simulation in the supply chain context: a survey." Computers in Industry, 53, pp. 3-16. 
Thornhill, N. F., and Naim, M. M. (2006). "An exploratory study to identify rogue seasonality in a steel companys supply network using spectral principal component analysis." European Journal of Operational Research, 172, pp. 146-162.

Ting, J. K., Ng, M. K., Rong, H., and Huang, J. Z. (2003). "Statistical models for time sequences data mining." I E E E International Conference on Computational Intelligence for Financial Engineering Hong Kong, China, 347-354.

Tong, H., and Dabas, P. (1990). "Cluster of time series models: An example." Journal of Applied Statistics, 17(2), pp. 187-198.

Torres, O. A. C., and Moran, F. A. V. (2006). "Managing the Bullwhip effect through Benchmarking " The Bullwhip effect in supply chains: A review of methods, components and cases, O. A. C. Torres and F. A. V. Moran, eds., Palgrave Macmillan, New York, 215-242.

Towill, D. R. (1970). Transfer Function Techniques for Control Engineers, Iliffe Books, London.

Towill, D. R. (1977). "Exponential smoothing of learning curves." International Journal Production Research 15, pp. 1-15.

Towill, D. R. (1982). "Dynamic analysis of an inventory and order based production control system." International Journal of Production Research, 20(6), pp. 671687.

Towill, D. R. (2005). "The impact of business policy on bullwhip induced risk in supply chain management." International Journal of Physical Distribution \& Logistics Management, 35(8), pp. 555-575.

Towill, D. R., Lambrecht, M. R., Disney, S. M., and Dejonckheere, J. (2003). "Explicit filters and supply chain design " Journal of Purchasing and Supply Management, 9(2), pp. 73-81.

Towill, D. R., and McCullen, P. (1999). "The impact of agile manufacturing on supply chain dynamics." International Journal of Logistics Management, 10(1), pp. 83-96.

Towill, D. R., Naim, M. M., and Wikner, J. (1992). "Industrial dynamics simulation models in the design of supply chains." International Journal of Physical Distribution \& Logistics Management, 22(5), pp. 3-13.

Towill, D. R., and Vecchio, A. d. (1994). "Application of filter theory to the study of supply chain dynamics." Production Planning and Control, 5(1), pp. 82-96.

Towill, D. R., Zhou, L., and Disney, S. M. (2007). "Reducing the bullwhip effect:Looking through the appropriate lens." International Journal of Production Economics, 108, pp. 444-453.

Tsung, F., Li, Y., and Jin, M. (2008). "Statistical process control for multistage manufacturing and service operations: a review and some extensions." International Journal of Services Operations and Informatics 3(2), pp. 191-204.

Tustin, A. (1953). The Mechanism of Economic Systems, William Heinemann, London.

Van Ackere, A., Larsen, E. R., and Morecroft, J. D. W. (1993). "Systems thinking and business process redesign: An application to the beer game." European Management Journal, 11(4), pp. 412-423.

Van Landeghem, H., and Vanmaele, H. (2002). "Robust planning: A new paradigm for demand chain planning." Journal of Operations Management, 20(6), pp. 769783.

Vassian, H. J. (1955). "Application of discrete variable servo theory to inventory control." Operations Research, 3(3), pp. 272-282. 
Venkatasubramanian, V., Rengaswamy, R., Yin, K., and Kavuri, S. N. (2003). "A review of process fault detection and diagnosis." Computers \& chemical engineering., 27(3), pp. 293-311.

Villegas, F. A., and Smith, N. R. (2006). "Supply chain dynamics: analysis of inventory vs. order oscillations trade-off." International Journal of Production Research, 44(6), pp. 1037-1054.

Vlachos, M., Yu, P. S., Castelli, V., and Meek, C. (2006). "Structural Periodic Measures for Time-Series Data." Data Mining And Knowledge Discovery, 12(1), pp. 1-28.

Wagner, S. M., and Bode, C. (2008). "An empirical examination of supply chain performance along several dimensions of risk." Journal of Business Logistics, 29(1), pp. 307-325.

Wang, C., and Wang, X. S. "Supporting content-based searches on time series via approximation." Proceedings of the 12th International Conference on Scientific and Statistical Database Management 69-81.

Wang, X., Smith, K., and Hyndman, R. (2006). "Characteristic-based clustering for time series data." Data Mining and Knowledge Discovery, 13(3), pp. 335-364.

Wikner, J., Naim, M. M. and Rudberg, M (2007), "Exploiting the order book for mass customized manufacturing control systems with capacity limitations", IEEE Transactions on Engineering Management, 54(1), pp.145-155

Wilding, R. (1998). "The supply chain complexity triangle: Uncertainty generation in the supply chain." International Journal of Physical Distribution \& Logistics Management, 28(8), pp. 599-616.

Wilson, M. C. (2007). "The impact of transportation disruptions on supply chain performance." Transportation Research Part E: Logistics and Transportation Review, 43(4), pp. 295-320.

Winston, A. (2005). Spreadsheet Modelling and Applications, Thomson, USA.

Woodall, W. H., and Montgomery, D. C. (1999). "Research issues and ideas in statistical process control." Journal of Quality Technology, 31(4), pp. 376.

$\mathrm{Wu}$, D. Y., and Katok, E. (2006). "Learning, communication, and the bullwhip effect." Journal of Operations Management, 24(6), pp. 839-850.

Wu, Y.-L., Agrawal, D., and Abbadi, A. E. (2000). "A comparison of DFT and DWT based similarity search in time-series databases." Proceedings of the ninth international conference on Information and knowledge management, ACM, McLean, Virginia, United States.

Xiong, Y., and Yeung, D.-Y. (2002). "Mixtures of ARMA Models for Model-Based Time Series Clustering." Proceedings of the 2002 IEEE International Conference on Data Mining (ICDM'02), IEEE Computer Society.

Yin, R. K. (1994). Case Study Research: Design and Methods. 2nd Ed, Sage Publications, London.

Zhang, H., Ho, T., and Huang, W. (2005). "Blind Feature Extraction for Time-Series Classification Using Haar Wavelet Transform." Advances in Neural Networks ISNN 2005, J. Wang, X. Liao, and Z. Yi, eds., Springer-Verlag, Berlin Heidelberg, 605-610.

Zhu, D., Premkumar, G., Zhang, X., and Chu, C. H. (2001). "Data mining for network intrusion detection: A comparison of alternative methods." Decision Sciences, 32(4), pp. 635-658.

Zsidisin, G. A. (2003). "Managerial perceptions of supply risk." Journal of Supply Chain Management, 39(1), pp. 14-25. 
Zsidisin, G. A., Ellram, L. M., Carter, J. R., and Cavinato, J. L. (2004). "An analysis of supply risk assessment techniques." International Journal of Physical Distribution \& Logistics Management, 34(5), pp. 397-413.

Zsidisin, G. A., Panelli, A., and Upton, R. (2000). "Purchasing organization involvement in risk assessments, contingency plans, and risk management: an exploratory study." Supply Chain Management: An International Journal, 5(4), pp. 187-197. 


\section{Appendix A}

\section{Block diagram and difference equations for a single echelon APIOBPCS system (John et al., 1994)}

Causal loop diagram of the APIOBPCS system given in Figure 3.2 is converted into an equivalent block diagram representation, a common approach in control theory (Nise, 1995) which clearly identifies the stock and flow structure. Difference equations are developed from this structure which are transferred to a Microsoft Excel spreadsheet for generation of the time series profiles of variables. Though the equations described here are for a single echelon, these can be coupled together to simulate multi echelon systems by making the ORATE of the downstream echelon equal to the demand signal of the upstream echelon. While the APIOBPCS model defined below is for the production echelon, it is valid for distribution echelons such as wholesaler, distributor also by appropriate modification of terms used (WIP in the distribution context refers to orders in the pipeline i.e. orders placed but against which goods have not yet been received, COMRATE refers to the rate at which orders get completed i.e. goods are received, $T_{p}$ refers to the delay or lead time between placing orders and receiving goods).

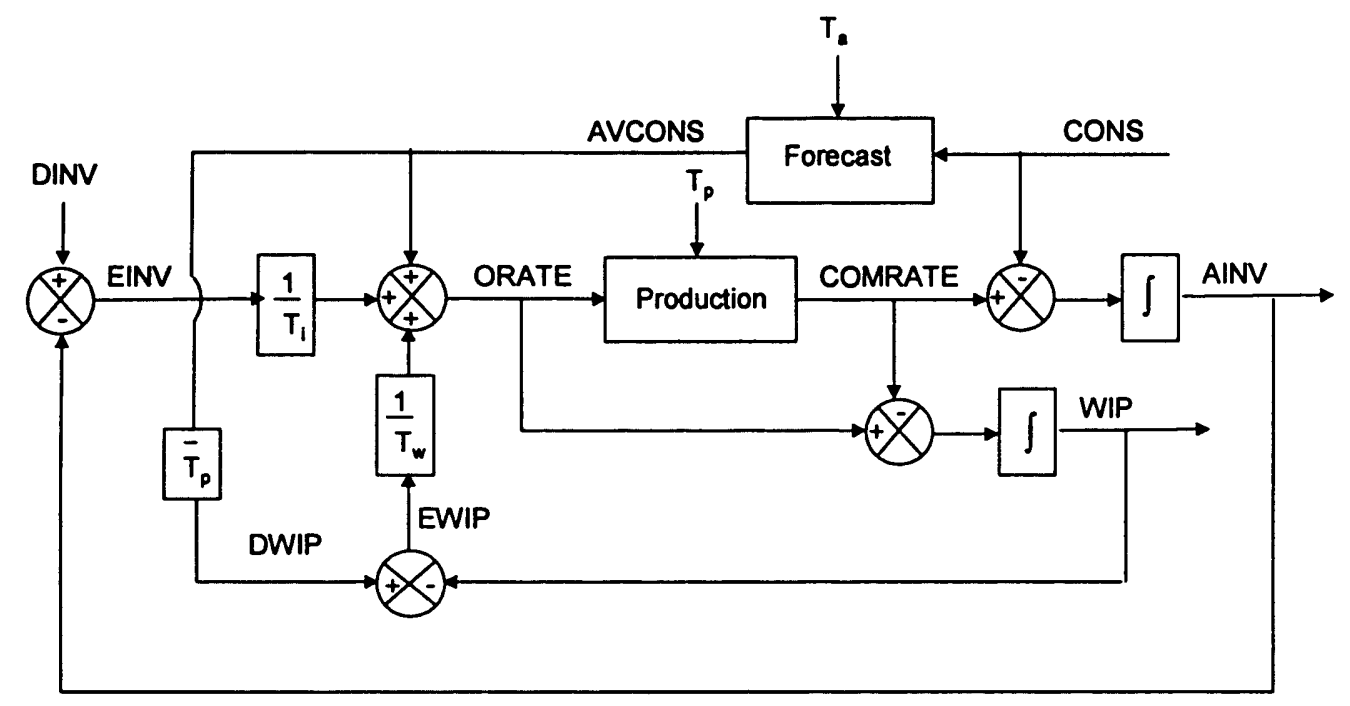

Forecast:

$\operatorname{AVCONS}(\mathrm{t})=\operatorname{AVCONS}(\mathrm{t}-1)+\alpha$ [CONS $(\mathrm{t})-\operatorname{AVCONS}(\mathrm{t}-1)]$ where; $\alpha=1 /\left(1+T_{a} / \Delta t\right)$, CONS $(t)$ is the demand at time $t$, and for a linear, time invariant system is a surrogate for sales, $T_{2}$ is the time to average demand, $\Delta t$ is our simulation time increment set at 1

Production Order Rate: ORATE $(t)=\operatorname{AVCONS}(t)+\left[\operatorname{EINV}(t-1) / T_{i}\right]+\left[\operatorname{EWIP}(t-1) / T_{w}\right]$ where; $T_{i}$ is the time to adjust inventory and $T_{w}$ the time to adjust work in process or WIP

Work in process level: WIP $(t)=$ WIP $(t-1)+\operatorname{ORATE}(t)-\operatorname{COMRATE}(t-1)$

Completion Rate/Receipts into inventory rate: COMRATE $(\mathrm{t})=\mathrm{WIP}(\mathrm{t}) / \mathrm{T}_{\mathrm{p}}-$ - First order lag COMRATE $(\mathrm{t})=$ ORATE $\left(\mathrm{t}+\mathrm{T}_{\mathrm{p}}\right)-$ Pure delay lag

where $T_{p}$ is the actual production lead time

Actual Inventory level: AINV $(t)=\operatorname{AINV}(t-1)+\operatorname{COMRATE}(t)-\operatorname{CONS}(t)$

Error in Inventory:

$\operatorname{EINV}(\mathrm{t})=$ DINV $-\operatorname{AINV}(\mathrm{t})$

where DINV is the desired or target inventory level (assumed constant)

Error in Work in Process or EWIP: EWIP $(t)=D W I P(t)-W I P(t)$

where; DWIP $(t)=\vec{T}_{p} * \operatorname{AVCONS}(t)$;

$\bar{T}_{p}$ is the estimated production lead time

Parameters suggested for good dynamic performance (John et al., 1994)

$T_{i}$ (time to adjust inventory) $=T_{p}$

$\mathrm{T}_{\mathrm{a}}$ (time to average demand) $=2 * \mathrm{~T}_{\mathrm{p}}$

$T_{w}$ (time to adjust work in progress or WIP) $=2 * T_{p}$

$\bar{T}_{p}$ (Estimated delay or production lead time) $=T_{p}$ 


\section{Appendix B}

Transfer functions for single echelon APIOBPCS (hybrid MTO-MTS), make to order (MTO) and make to stock (MTS) systems

APIOBPCS system: Obtained from block diagram in Appendix A

$\frac{A V C O N S}{\operatorname{CONS}}=\frac{1}{1+T_{a} s}---\left(\right.$ B.1) $\quad$ DWIP $=T_{p}($ AVCONS $)--\left(\right.$ B.2) $\quad$ AINV $=\frac{1}{s}($ COMRATE - CONS $)--$ (B.3)

$E W I P=D W I P-W I P---(\mathbf{B . 4})$

$\frac{\text { COMRATE }}{\text { ORATE }}=\frac{1}{1+T_{p^{s}}}($ Order 1$)$ or $=\mathrm{e}^{-T p s}$ (for order infinity) $\approx \frac{\left(s T_{p}\right)^{2}-6\left(s T_{p}\right)+12}{\left(s T_{p}\right)^{2}+6\left(s T_{p}\right)+12} \begin{gathered}\left(2^{\text {nd }} \text { order Pade's approx. }\right)---(\text { B.5) } \\ \text { (Nise, 1995) }\end{gathered}$

$W I P=\frac{1}{s}($ ORATE - COMRATE $)-(\mathbf{B . 6}) \quad E I N V=D I N V-A I N V-\mathbf{( B . 7 )} \quad$ ORATE $=A V C O N S+\frac{E W I P}{T_{w}}+\frac{E I N V}{T_{i}}-\mathbf{( B . 8 )}$

MTO system: Parameter values $T_{a}=0, T_{i}=\infty, T_{w}=\infty$ substituted into Equations 1 to 8 and solved

\section{Order of delay 1}

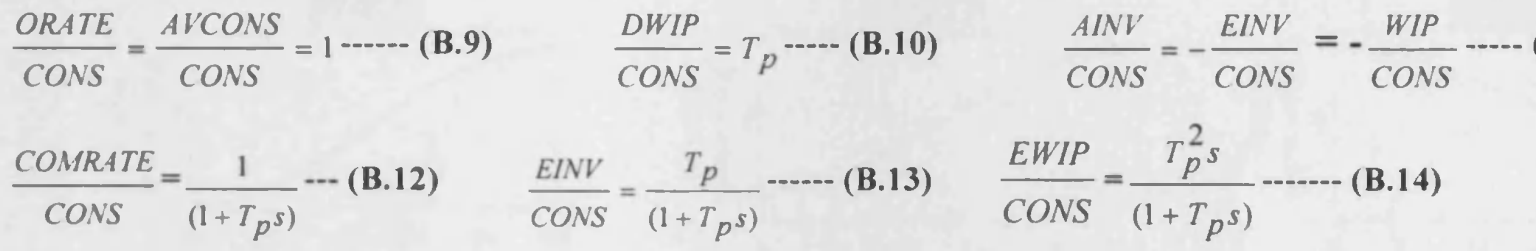

\section{Order of delay infinity}

Equations (9), (10) and (11) are the same as in above

$$
\frac{\text { COMRATE }}{\operatorname{CONS}}=\frac{\left(12-6 T_{p} s+T_{p}^{2} s^{2}\right)}{\left(12+6 T_{p} s+T_{p}^{2} s^{2}\right)}-- \text { (B.15) } \frac{E I N V}{\operatorname{CONS}}=\frac{12 T_{p}}{\left(12+6 T_{p} s+T_{p}^{2} s^{2}\right)}-- \text { (B.16) } \frac{E W I P}{\operatorname{CONS}}={\frac{T_{p}^{3} s^{2}+6 T_{p}^{2} s}{\left(12+6 T_{p} s+T_{p}^{2} s^{2}\right)}}^{---(\text {B.17) }}
$$

MTS system: Parameter values $T_{a}=\infty, T_{i}=T_{p}, T_{w}=\infty$ substituted into Equations 1 to 8 and solved.

\section{Order of delay 1}

$$
\begin{aligned}
& \frac{A V C O N S}{C O N S}=\frac{D W I P}{C O N S}=0---- \text { (B.18) } \quad \frac{A I N V}{C O N S}=-\frac{E I N V}{C O N S}=-T_{p} \frac{\text { ORATE }}{C O N S}--- \text { (B.19) } \quad \frac{E W I P}{C O N S}=-\frac{W I P}{C O N S}--- \text { (B.20) } \\
& \frac{\text { ORATE }}{\text { CONS }}=\frac{\left(1+T_{p} s\right)}{\left(1+T_{p} s+T_{p}^{2} s^{2}\right)}-\cdots \text { (B.21) } \quad \frac{\text { COMRATE }}{C O N S}=\frac{1}{\left(1+T_{p} s+T_{p}^{2} s^{2}\right)}-\text { (B.22) } \quad \frac{W I P}{C O N S}=\frac{T_{p}}{\left(1+T_{p} s+T_{p}^{2} s^{2}\right)}-
\end{aligned}
$$

\section{Order of delay infinity}

Equations (18), (19) and (20) are the same as in above

$$
\begin{aligned}
& \frac{\text { ORATE }}{\operatorname{CONS}}=\frac{\left(12+6 T_{p} s+T_{p}^{2} s^{2}\right)}{\left(12+6 T_{p} s+7 T_{p}^{2} s^{2}+T_{p}^{3} s^{3}\right)}---\left(\text { B.24) } \quad \frac{\text { COMRATE }}{\text { CONS }}=\frac{\left(12-6 T_{p} s+T_{p}^{2} s^{2}\right)}{\left(12+6 T_{p} s+7 T_{p}^{2} s^{2}+T_{p}^{3} s^{3}\right)}-\cdots\right. \\
& \frac{12 T_{p}}{\operatorname{CONS}}=\frac{{ }^{---}}{\left(12+6 T_{p} s+7 T_{p}^{2} s^{2}+T_{p}^{3} s^{3}\right)}--(\text { (B.26) }
\end{aligned}
$$




\section{Appendix C}

\section{Theoretical spectral density plots for different $\mathrm{AR}$ and MA time series processes}

Spectral density for an MA $(q)$ process defined by $X_{t}=\varepsilon_{t}-\theta_{1} \varepsilon_{t-1}-\theta_{2} \varepsilon_{t-2}-------\theta_{q} \varepsilon_{t-q}$ can be analytically derived and represented as

Spectral density $-p_{x}(f)=\left(\sigma_{a}{ }^{2} / 2 \pi\right)\left(\left(1+\theta_{1}{ }^{2}+\theta_{2}{ }^{2}+----\theta_{q}{ }^{2}\right)+\left(-\theta_{1}+\theta_{1} \theta_{2}+\theta_{2} \theta_{3}+---\theta_{q-1} \theta_{q}\right) 2 \cos \lambda+\right.$

$\left.\left(-\theta_{2}+\theta_{1} \theta_{3}+\theta_{2} \theta_{1}+-----\theta_{q-2} \theta_{q}\right) 2 \cos 2 \lambda+-----\left(-\theta_{q-1}+\theta_{l} \theta_{q}\right) 2 \cos (q-1) \lambda\right)$

- Spectral density at a particular frequency is proportional to the square of the amplitude at that frequency

- $\quad \lambda=2 \pi f$ where $f=$ frequency

- $\quad \sigma_{\theta}{ }^{2}=$ Variance of the white noise process

- $\theta_{1}, \theta_{2}$----- $\theta_{4}$ are the parameters of the MA process

For MA (1) process $p_{x}(f)=\left(1+\theta^{2}-2 \theta \cos 2 \pi f\right)\left(\sigma_{s}{ }^{2} / 2 \pi\right)$

For MA (2) process $p_{x}(f)=\left(1+\theta_{1}{ }^{2}+\theta_{2}{ }^{2}+\left(-\theta_{1}+\theta_{1} \theta_{2}\right) 2 \cos \lambda-\left(\theta_{2}\right) 2 \cos 2 \lambda\right)\left(\sigma_{\theta}{ }^{2} / 2 \pi\right)----($ C.3 $)$

Since any MA (q) process can be equivalently represented in terms of an AR (p) process, the spectral density for the latter can be derived. An $\mathrm{AR}(\mathrm{p})$ is represented by $X_{t}=\varepsilon_{1}+\rho_{l} X_{t-1}+\rho_{2} X_{t-2}+---\rho_{p} X_{t-p}$ where $\rho$ 's are the parameters of the AR model.

For AR (1) process $p_{x}(f)=\left(1 /\left(1+\rho^{2}-2 \rho \cos \lambda\right)\right)\left(\sigma_{e}^{2} / 2 \pi\right)$

For AR (2) process $p_{x}(f)=\left(1 /\left(\left(1+\rho_{1}{ }^{2}+\rho_{2}{ }^{2}\right)-2 \rho_{1}\left(1-\rho_{2}\right) \cos 2 \pi f-2 \rho_{2} \cos 4 \pi f\right)\right)\left(\sigma_{e}{ }^{2} / 2 \pi\right)$
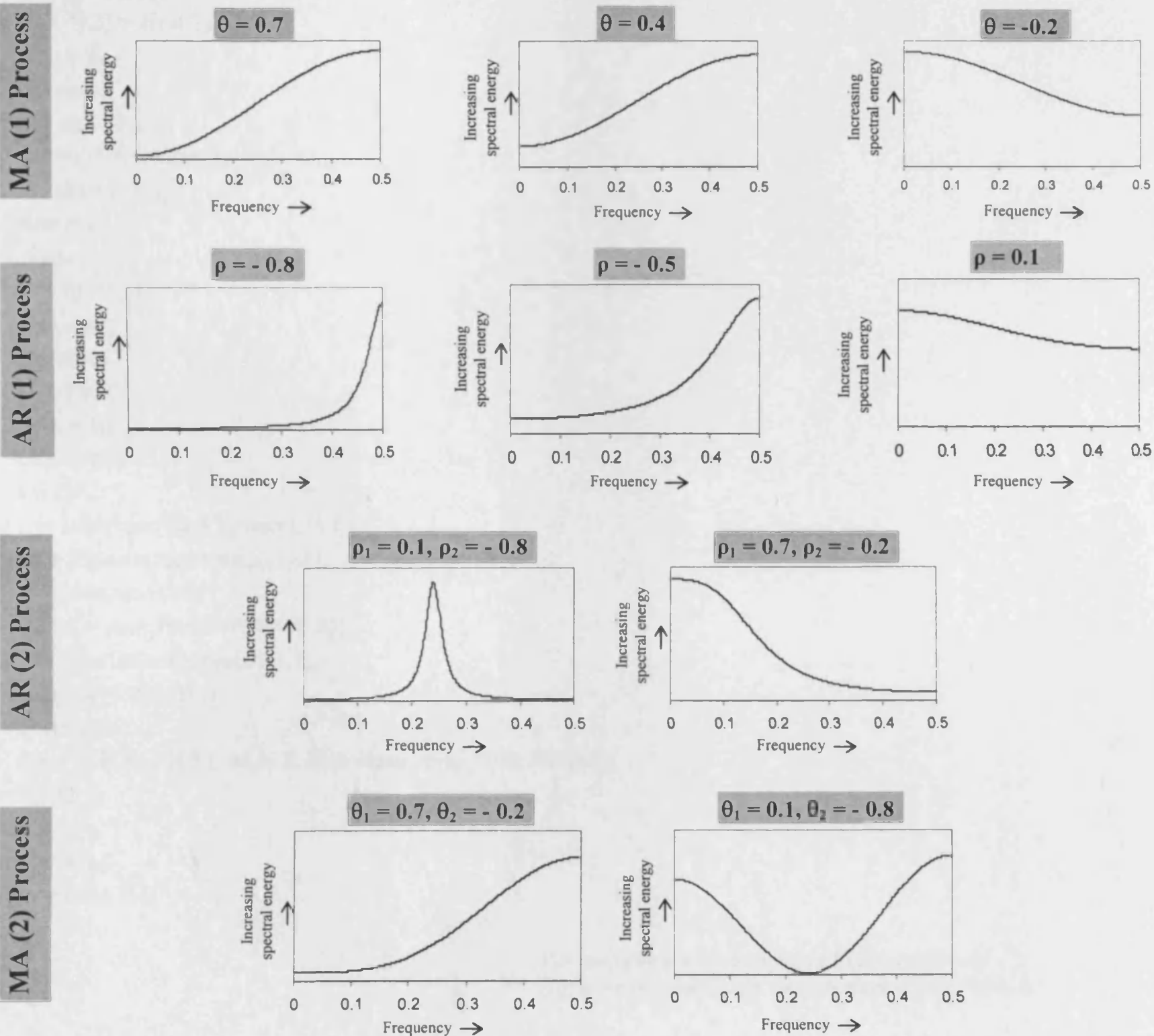


\section{Appendix D}

Matlab code for different time series transformations and use of relevant features** for computation* of the rogue seasonality index

\section{Raw time and Fourier transform (FT)}

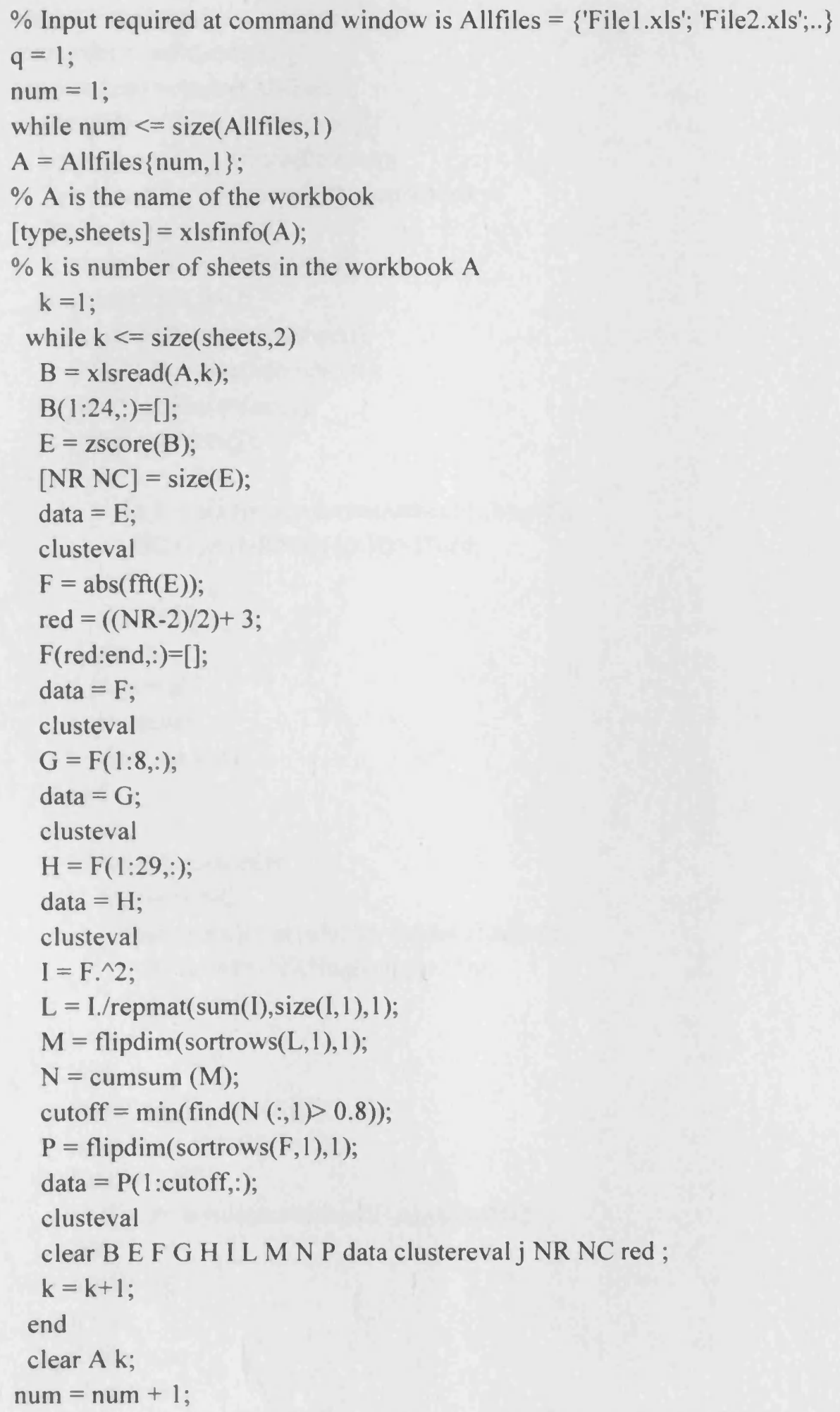




\section{Appendix D continued}

\section{Autoregressive model (AR Model)}

$\%$ Input reqd at command window is Allfiles $=\{$ 'Filel.xls'; 'File2.xls';.. $\}$

user_entry = input('Input AR orders to be considered with gaps between the numbers ', 's');

$\mathrm{q}=1$

orders $=$ str2num(user_entry);

maxorder $=\max ($ orders, []$, 2)$;

for numfiles $=1: \operatorname{size}($ Allfiles, 1$)$

filenme $=$ Allfiles $\{$ numfiles, 1$\}$;

[type, sheets] $=$ xlsfinfo(filenme);

$\% i$ is number of sheets in the workbook $A$

for $\mathrm{i}=1$ :size(sheets, 2 )

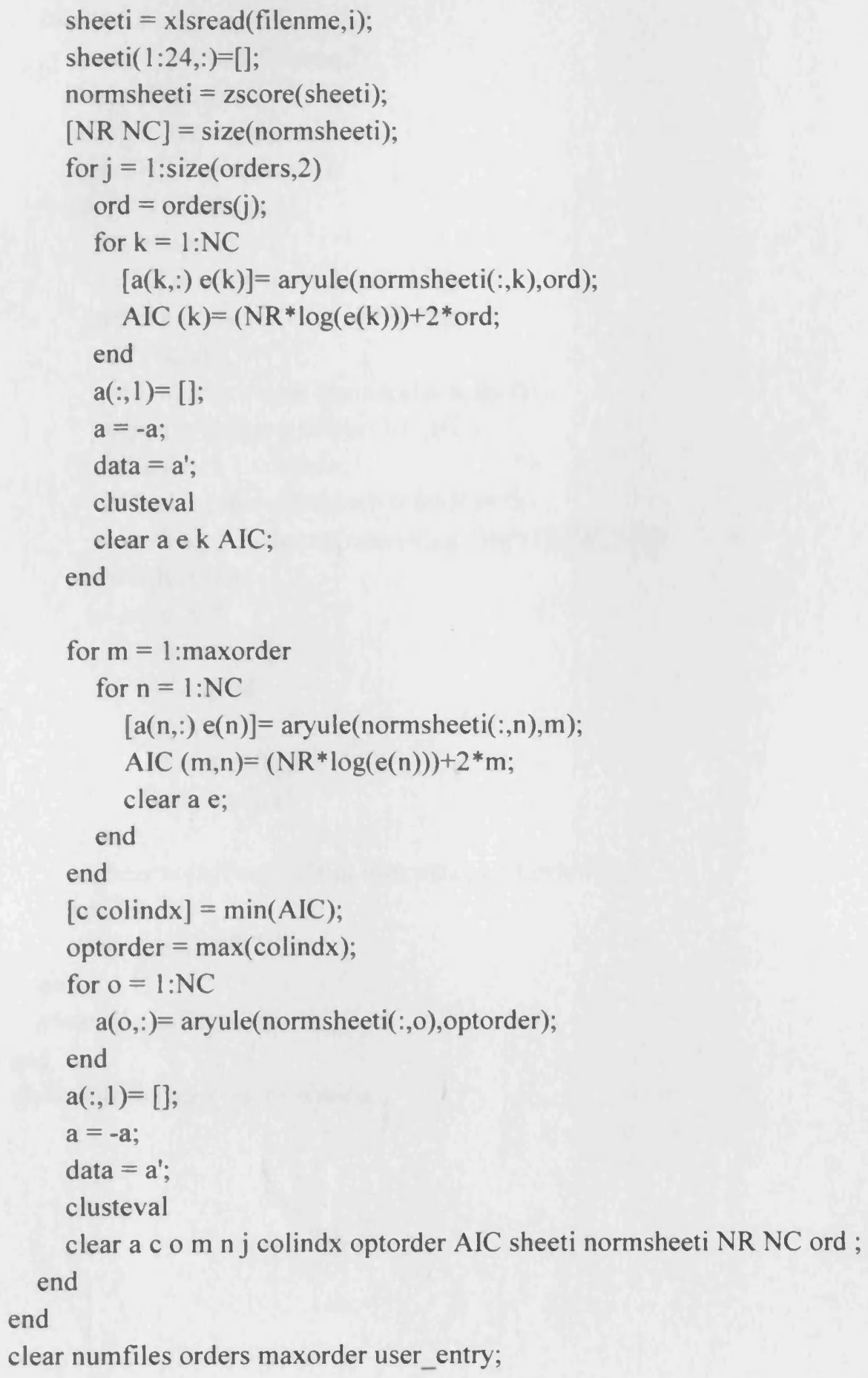




\section{Appendix D continued}

\section{Autocorrelation function (ACF) and Cross correlation function (CCF)}

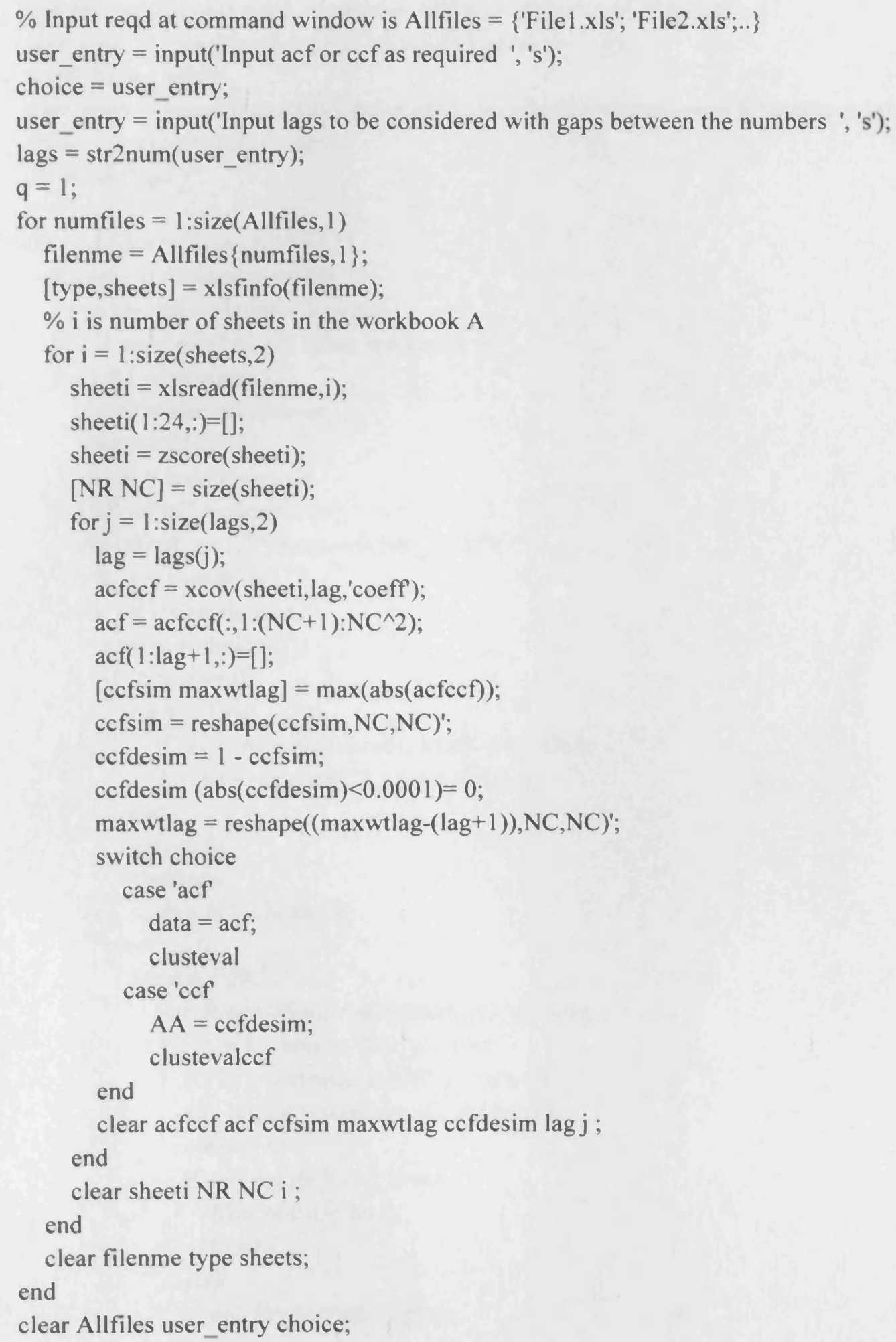




\section{Appendix D continued}

\section{Discrete wavelet transform (DWT) - Haar}

$\%$ Input reqd at command window is Allfiles $=\{$ 'Filel.xls'; 'File2.xls';... $\}$

user_entry = input('Input type of wavelet to consider ', 's');

wvlet = user_entry;

user_entry = input('Input different levels to be considered with gaps; 0 for min shannon basis ', 's');

choices $=$ str2num(user_entry);

dwtmode('zpd');

$\mathrm{q}=1$

for numfiles $=1$ :size (Allfiles, 1$)$

filenme $=$ Allfiles $\{$ numfiles, 1$\}$;

[type, sheets] $=$ xlsfinfo(filenme);

$\% \mathrm{i}$ is number of sheets in the workbook $\mathrm{A}$

for $i=1$ :size(sheets, 2 )

sheet $=$ xlsread $($ filenme, $\mathrm{i})$;

sheeti $(1: 24,:)=[]$;

sheeti $=$ zscore(sheeti);

[NR_o NC] $=$ size(sheeti);

sheeti(NR_o+1:2^nextpow2(NR_o),:) $=0$;

NR_n $=$ size(sheeti, 1);

for $\mathbf{j}=1$ : $\operatorname{size}($ choices, 2 )

choice $=\operatorname{choices}(\mathrm{j})$;

if choice $=0$

for $\mathrm{k}=1 \mathrm{NC}$

$[C \mathrm{~L}]=$ wavedec(sheeti(:,k), choice, wvlet);

A $(:, \mathrm{K})=\operatorname{appcoef}(\mathrm{C}, \mathrm{L}, \mathrm{wvlet}, \mathrm{choice})$;

end

data $=\mathrm{A}$;

clusteval

clear $\mathrm{k}$ C L choice A;

else

for $\mathrm{n}=1: \mathrm{NC}$

ent_lower = wentropy $($ sheeti $(:, n)$, 'shannon');

for $m=1$ :wmaxlev(NR_n,wvlet)

$[C \mathrm{~L}]=$ wavedec(sheeti(:,n),m,wvlet);

ent_higher = wentropy $(C$,'shannon');

clear C L;

if ent_higher > ent_lower

Min_ent $(n)=m-1$;

break

else

ent_lower = ent_higher;

end

end

clear m ent_higher ent_lower;

end

if $\min ($ Min_ent $)=0$

common_ent $=$ round $($ median $($ Min_ent $))$;

else 


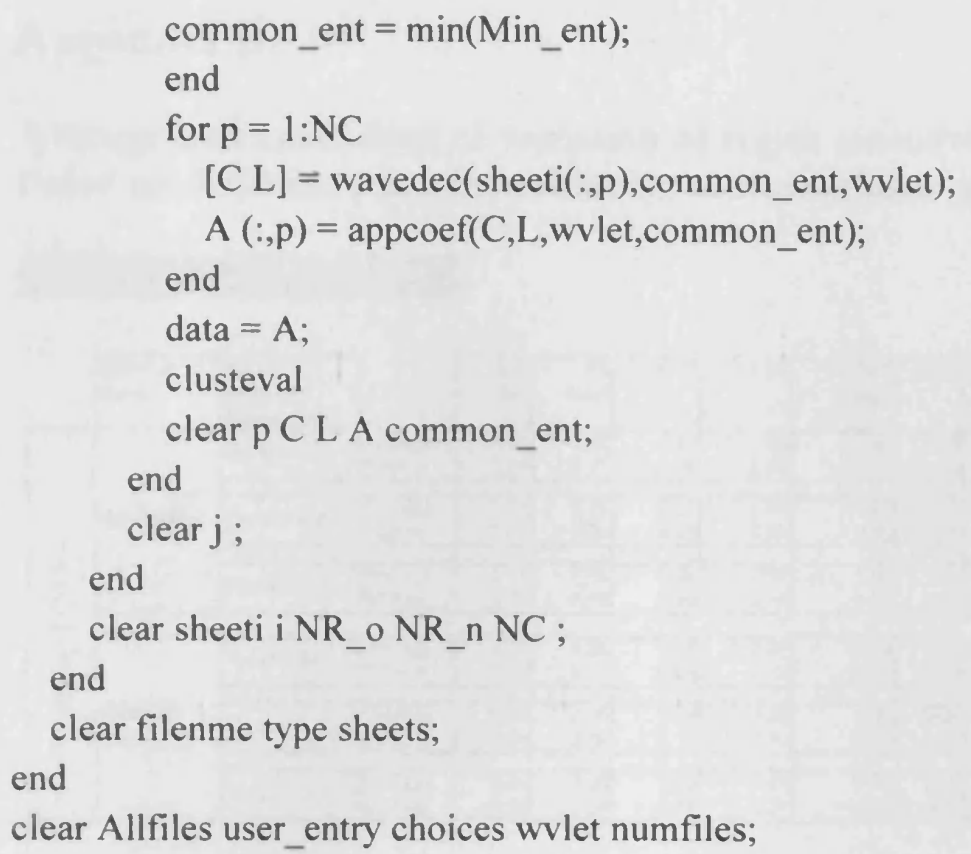

\section{Rogue seasonality index computation^ (Clusteval)}

$\mathrm{AA}=$ squareform(pdist(data'));

$\mathrm{AA}(\mathrm{AA}==0)=0.000000001$;

$\mathrm{BA}=\mathrm{AA}$;

$\operatorname{BA}(1,:)=[]$

$\mathrm{BA}(:, 1)=[]$;

$\mathrm{XA}=\operatorname{mean}(\mathrm{BA}($ find $(\operatorname{triu}(\mathrm{BA}, 1))))$;

$Z A=\operatorname{std}(B A($ find $(\operatorname{triu}(B A, 1))))$;

Alt $A=\min (A A(1,2$ :end $)) / X A$;

$\mathrm{AltB}=\operatorname{mean}(\mathrm{AA}(1,2$ :end $)) / \mathrm{XA}$;

$\mathrm{AltC}=(\min (\mathrm{AA}(1,2:$ end $))-\mathrm{XA}) / \mathrm{ZA}$;

evaltotal $(q,:)=([$ Alt $A, A l t B, A l t C])$;

$q=q+1$

q

clear data AA BA XA ZA AltA AltB AltC;

${ }^{\wedge}$ Same for all except cross correlation function (CCF) for which first line of the code is removed 


\section{Appendix E}

Average and coefficient of variation of rogue seasonality index values for a 3 echelon MTS system based on different feature parameter combinations; All variables used in the index computation

\section{Demand Process: MA (1)}

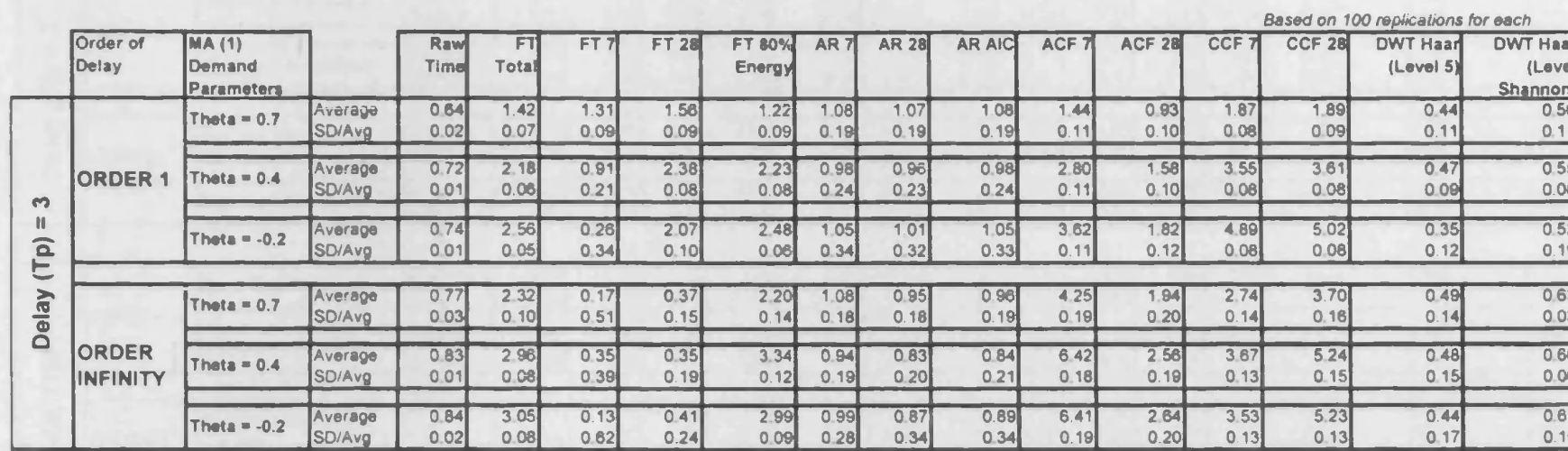

\begin{tabular}{|c|c|c|c|c|c|c|c|c|c|c|c|c|c|c|c|c|c|}
\hline \multirow{10}{*}{ 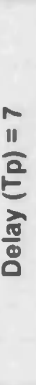 } & \multirow{4}{*}{ ORDER 1} & Thete $=0.7$ & \begin{tabular}{|l}
$\begin{array}{l}\text { Average } \\
\text { SOrAvg }\end{array}$ \\
\end{tabular} & $\begin{array}{l}0.79 \\
0.03 \\
\end{array}$ & $\begin{array}{l}1.89 \\
0.10 \\
\end{array}$ & $\begin{array}{l}2.20 \\
0.43 \\
\end{array}$ & $\begin{array}{l}237 \\
0.13 \\
\end{array}$ & $\begin{array}{l}1.82 \\
0.12 \\
\end{array}$ & $\begin{array}{r}1.97 \\
0.32 \\
\end{array}$ & $\begin{array}{r}1.93 \\
0.28 \\
\end{array}$ & $\begin{array}{l}2.00 \\
0.31 \\
\end{array}$ & $\begin{array}{l}2.23 \\
0.18 \\
\end{array}$ & $\begin{array}{l}.24 \\
0.18 \\
\end{array}$ & $\begin{array}{l}2.99 \\
0.12 \\
\end{array}$ & $\begin{array}{l}281 \\
0.14 \\
\end{array}$ & $\begin{array}{l}0.51 \\
0.12 \\
\end{array}$ & $\begin{array}{l}0.55 \\
0.07 \\
\end{array}$ \\
\hline & & Theta $=0.4$ & $\begin{array}{l}\text { Average } \\
\text { SDrAvo }\end{array}$ & 0.87 & 3.02 & 2.48 & 3.41 & 3.46 & 1.88 & 1.77 & 1.87 & 5.92 & 236 & 4.02 & 6.17 & 0.59 & $\begin{array}{l}0.66 \\
0.08\end{array}$ \\
\hline & & & Average & 0.90 & 331 & 167 & 307 & 325 & 203 & 190 & 2031 & 840 & 268 & 448 & 769 & 051 & \\
\hline & & Thote $=-0.2$ & SD/Avg & 0.01 & 0.08 & 0.27 & 0.09 & 0.08 & 0.51 & 0.45 & 0.52 & 0.19 & 0.17 & 0.09 & 0.12 & 0.09 & 0.17 \\
\hline & & & & & & & & & & & & & & & & & \\
\hline & \multirow{5}{*}{$\begin{array}{l}\text { ORDER } \\
\text { INFINITY }\end{array}$} & Thota $=0.7$ & $\begin{array}{l}\text { Average } \\
\text { SO/Avg }\end{array}$ & $\begin{array}{l}0.79 \\
0.03\end{array}$ & $\begin{array}{l}2.40 \\
0.12\end{array}$ & $\begin{array}{l}0.38 \\
0.41\end{array}$ & $\begin{array}{l}3.04 \\
0.14\end{array}$ & $\begin{array}{l}2.49 \\
0.14\end{array}$ & $\begin{array}{l}2.07 \\
0.25\end{array}$ & $\begin{array}{l}1.57 \\
0.18\end{array}$ & $\begin{array}{l}1.57 \\
0.18\end{array}$ & $\begin{array}{l}5.17 \\
021\end{array}$ & $\begin{array}{l}2.41 \\
024\end{array}$ & $\begin{array}{l}2.40 \\
0.17\end{array}$ & $\begin{array}{l}3.44 \\
0.18\end{array}$ & $\begin{array}{l}0.52 \\
0.11\end{array}$ & $\begin{array}{l}0.63 \\
0.04\end{array}$ \\
\hline & & Thate 0 & $\operatorname{AI}_{2}$ & 0.89 & 3.44 & $\overline{0.20}$ & 3.41 & 4.27 & 181 & 1.43 & 1.44 & 16.70 & 3.99 & 3.64 & 5.75 & 0.47 & 089 \\
\hline & & Thote 0.8 & SD/Avg & 0.01 & 0.11 & 0.40 & 0.13 & 0.18 & 0.28 & 0.27 & 0.28 & 0.21 & 0.25 & 0.18 & 0.17 & 0.13 & 0.06 \\
\hline & & Thote $=-0.2$ & 90 & 0.91 & 3.58 & 0.25 & 2.90 & 359 & 1.98 & 1.78 & 1.80 & 30.44 & 431 & 3.70 & 807 & 0.46 & 0.70 \\
\hline & & Thine -.8 & SD/Avg & 0.01 & 0.11 & 0.48 & 0.13 & 0.11 & 0.43 & 0.43 & 0.48 & 0.20 & 0.26 & 0.15 & 0.18 & 0.14 & 0.15 \\
\hline
\end{tabular}

\begin{tabular}{|c|c|c|c|c|c|c|c|c|c|c|c|c|c|c|c|c|c|}
\hline \multirow{11}{*}{$\begin{array}{l}\frac{a}{0} \\
\frac{a}{2} \\
\frac{a}{0} \\
\frac{\pi}{0}\end{array}$} & \multirow{5}{*}{ ORDER 1} & Theta $=0.7$ & \begin{tabular}{|l}
$\begin{array}{l}\text { Averago } \\
\text { SO/Avg }\end{array}$ \\
\end{tabular} & $\begin{array}{l}0.83 \\
0.03 \\
\end{array}$ & $\begin{array}{l}2.44 \\
0.13 \\
\end{array}$ & $\begin{array}{l}2777 \\
0.20 \\
\end{array}$ & $\begin{array}{l}2.85 \\
0.15 \\
\end{array}$ & $\begin{array}{l}2.74 \\
0.17 \\
\end{array}$ & $\begin{array}{l}2.94 \\
0.22 \\
\end{array}$ & $\begin{array}{l}2.80 \\
0.18 \\
\end{array}$ & $\begin{array}{l}2.86 \\
0.20 \\
\end{array}$ & $\begin{array}{l}4.21 \\
0.25 \\
\end{array}$ & $\begin{array}{l}2.30 \\
0.26 \\
\end{array}$ & $\begin{array}{l}2.27 \\
0.13 \\
\end{array}$ & $\begin{array}{l}399 \\
0.19 \\
\end{array}$ & $\begin{array}{l}0.58 \\
0.08 \\
\end{array}$ & $\begin{array}{l}0.63 \\
0.07 \\
\end{array}$ \\
\hline & & Thet $=0.4$ & Average & 0.95 & 3.61 & 375 & 336 & 4.81 & 273 & 257 & 271 & 11.77 & 428 & 3.45 & 7.78 & 0.64 & 0.73 \\
\hline & & Thets - 0 & SD/Avg & 0.02 & 0.12 & 0.18 & 0.12 & 0.17 & 0.26 & 0.25 & 0.26 & 0.25 & 0.25 & 0.11 & 0.17 & 0.06 & 0.07 \\
\hline & & Thete $=\mathbf{- 0 . 2}$ & Average & 0.98 & 3.77 & 3.54 & 2.94 & 3.77 & 3.62 & 3.08 & 3.48 & 18.72 & 476 & 3.57 & 887 & 060 & 0.74 \\
\hline & & De & Soravg & 0.01 & 0.11 & 0.17 & 0.11 & 0.12 & 0.55 & 0.45 & 0.53 & 0.25 & 0.25 & 0.10 & 0.16 & 0.08 & 0.16 \\
\hline & \multirow{6}{*}{$\begin{array}{l}\text { ORDER } \\
\text { INFINITY }\end{array}$} & Theta $=0.7$ & Avertage & 0.84 & 288 & 1.84 & 3.25 & 3.49 & 2.83 & 231 & 233 & 7.50 & 441 & 221 & 401 & 0.60 & 0.87 \\
\hline & & & SO/Avg & 0.03 & 0.17 & 0.41 & 0.17 & 0.20 & 0.18 & 0.15 & 0.15 & 0.31 & 0.31 & 0.19 & 024 & 0.11 & 0.04 \\
\hline & & Thote 0.4 & Average & 0.94 & 3.97 & 1.26 & 345 & 5.62 & 257 & 2.16 & 2.18 & 24.12 & 7.69 & 301 & 613 & 0.81 & 0.73 \\
\hline & & & SO/Avg & 0.01 & 0.16 & 0.48 & 0.18 & 0.24 & 025 & 025 & & 0.31 & 0.321 & 0.18 & 0.22 & 013 & 0.07 \\
\hline & & Thota $=-0.2$ & Averago & 097 & 000 & 089 & 302 & 4.14 & 3.10 & 259 & 271 & 3987 & 8.35 & 304 & 631 & 0.57 & 0.74 \\
\hline & & & SOS/Avg & $0.01]$ & 0.15 & 0.54 & 0.15 & 0.15 & 0.47 & 0.39 & 0.42 & 032 & $033]$ & 0.15 & 020 & 0.13 & \\
\hline
\end{tabular}

\section{Demand Process: Gaussian random}

\begin{tabular}{|c|c|c|c|c|c|c|c|c|c|c|c|c|c|c|c|c|}
\hline & $\begin{array}{l}\text { Order of } \\
\text { Dolay }\end{array}$ & & $\begin{array}{l}\text { Ramp } \\
\text { Time }\end{array}$ & Total & FTT & FT 2ब & $\begin{array}{l}\text { FT 80\% } \\
\text { Energy }\end{array}$ & AR T & AR 2ब & AR AIC & ACF T & ACF 28 & CCF & CCF 26 & $\begin{array}{l}\text { DWT Haas } \\
\text { (Lovel B) }\end{array}$ & $\begin{array}{l}\text { DWT Haer } \\
\text { (Love } \\
\text { Shennon }\end{array}$ \\
\hline \multirow{3}{*}{ 离 $\frac{m}{a}$} & ORDER 1 & $\begin{array}{l}\text { Average } \\
\text { SD/Avg }\end{array}$ & $\begin{array}{l}0.75 \\
0.01\end{array}$ & $\begin{array}{l}260 \\
005\end{array}$ & $\begin{array}{l}023 \\
0.50\end{array}$ & $\begin{array}{l}229 \\
0.10\end{array}$ & $\begin{array}{l}2.62 \\
0.06\end{array}$ & \begin{tabular}{l|}
1.04 \\
0.34
\end{tabular} & $\begin{array}{l}1.00 \\
033\end{array}$ & $\begin{array}{l}103 \\
034\end{array}$ & $\begin{array}{l}3.88 \\
0.11\end{array}$ & $\begin{array}{l}9.87 \\
0.11\end{array}$ & $\begin{array}{l}5.18 \\
0.06\end{array}$ & $\begin{array}{l}5.31 \\
0.06\end{array}$ & $\begin{array}{l}039 \\
0.11\end{array}$ & $\begin{array}{l}0.52 \\
0.17\end{array}$ \\
\hline & ORDER & Average & 085 & 3.14 & 0.25 & 0.59 & 3.19 & 0.94 & 0.82 & 0.84 & 6.74 & 269 & 3.88 & 5.72 & 0.46 & 0.65 \\
\hline & INFINITY & SD/Avg & 0.01 & 0.08 & 0.53 & 0.16 & 0.09 & 0.25 & 0.29 & 0.29 & 0.19 & 020 & 0.12 & 0.13 & 0.14 & 0.11 \\
\hline \multirow{3}{*}{ 要 $\frac{\pi}{\text { व }}$} & ORDER 1 & $\begin{array}{l}\text { Average } \\
\text { Solavg }\end{array}$ & $\begin{array}{l}0.90 \\
0.01\end{array}$ & $\begin{array}{l}3.38 \\
0.08\end{array}$ & $\begin{array}{l}1.91 \\
0.24\end{array}$ & $\begin{array}{l}3.25 \\
0.09\end{array}$ & $\begin{array}{l}3.45 \\
0.09\end{array}$ & $\begin{array}{l}1.93 \\
0.41 \\
\end{array}$ & $\begin{array}{l}.82 \\
0.38\end{array}$ & $\begin{array}{r}193 \\
0.42\end{array}$ & $\begin{array}{l}8.22 \\
0.19\end{array}$ & $\begin{array}{l}2.69 \\
0.17\end{array}$ & $\begin{array}{l}4.82 \\
0.09\end{array}$ & $\begin{array}{l}8.16 \\
0.12\end{array}$ & $\begin{array}{l}0.54 \\
0.09\end{array}$ & $\begin{array}{l}0.85 \\
0.15\end{array}$ \\
\hline & ORDER & Average & 0.92 & 366 & 0.24 & 3.08 & 3.81 & 1.82 & 1.69 & 1.72 & 28.48 & 4.34 & 3.92 & 6.42 & 0.46 & 0.68 \\
\hline & INFINITY & SDIAvg & 0.09 & 0.11 & 0.44 & 0.13 & 0.12 & 039 & 0.40 & 0.43 & 0.20 & 0.26 & 0.15 & $0.1 \mathrm{~d}$ & 0.14 & 0.15 \\
\hline \multirow{3}{*}{ 要告 } & ORDER 1 & Average & 0.98 & 3.84 & 3.67 & 3.09 & $\begin{array}{l}4.01 \\
0.13\end{array}$ & $\begin{array}{l}3.61 \\
0.57\end{array}$ & $\begin{array}{l}3.201 \\
0.88\end{array}$ & $\begin{array}{l}3.555 \\
0.55\end{array}$ & $\begin{array}{r}16.27 \\
0.29\end{array}$ & $\begin{array}{l}4.76 \\
0.25\end{array}$ & 3.77 & 9.25 & 0.62 & 0.72 \\
\hline & ORDER & $\widehat{A \text { Average }}$ & 0.97 & $\overline{4.13}$ & 0.96 & 3.15 & 4.39 & 3.43 & 2.77 & 2.89 & 37.75 & 8.38 & 3.16 & 6.57 & 0.58 & 0.72 \\
\hline & INFINITY & SD/Avg & 001 & 0.15 & 0.55 & 0.15 & 0.16 & 0.54 & 0.43 & 0.46 & 0.32 & 0.33 & 0.15 & 0.20 & 0.13 & 0.15 \\
\hline
\end{tabular}




\section{Appendix E continued}

\section{Demand Process: AR (2)}

\begin{tabular}{|c|c|c|c|c|c|c|c|c|c|c|c|c|c|c|c|c|c|}
\hline & $\begin{array}{l}\text { Drder of } \\
\text { Delay }\end{array}$ & $\begin{array}{l}\text { AR (2) Domand } \\
\text { Parameters }\end{array}$ & & $\begin{array}{l}\text { Raw } \\
\text { Timed }\end{array}$ & $\begin{array}{l}\text { Fr } \\
\text { Total }\end{array}$ & FT 7 & FT 2E & $\begin{array}{l}\text { FT 800 } \\
\text { Energy }\end{array}$ & AR 7 & AR 28 & AR AIC & ACF & ACF 28 & CCF & CCF 28 & $\begin{array}{c}\begin{array}{c}\text { DWT Has. } \\
\text { (Lovel 5) }\end{array} \\
\end{array}$ & $\begin{array}{l}\text { DWT Haan } \\
\text { ILove } \\
\text { Shannon }\end{array}$ \\
\hline \multirow{4}{*}{$\begin{array}{l}\text { m } \\
\frac{a}{2} \\
\frac{\pi}{0}\end{array}$} & \multirow{2}{*}{ ORDER 1} & Rho1 $=0.1$ Rho2 $=-0.8$ & $\begin{array}{l}\text { Averago } \\
\text { SD/Avg }\end{array}$ & $\begin{array}{l}0.70 \\
0.01\end{array}$ & $\begin{array}{l}1.28 \\
0.08\end{array}$ & $\begin{array}{l}1.28 \\
0.11 \\
\end{array}$ & $\begin{array}{l}1.60 \\
0.10\end{array}$ & $\begin{array}{l}0.80 \\
0.15\end{array}$ & $\begin{array}{l}0.84 \\
0.25\end{array}$ & $\begin{array}{l}0.82 \\
0.24\end{array}$ & $\begin{array}{l}0.84 \\
0.25 \\
\end{array}$ & $\begin{array}{l}1.31 \\
0.15 \\
\end{array}$ & $\begin{array}{l}0.93 \\
0.12\end{array}$ & $\begin{array}{l}208 \\
0.00\end{array}$ & $\begin{array}{l}2.08 \\
0.09\end{array}$ & $\begin{array}{l}0.43 \\
0.11\end{array}$ & $\begin{array}{l}0.50 \\
0.18\end{array}$ \\
\hline & & Rho1 $=0.7$ Rho2 $=-0.2$ & $\begin{array}{l}\text { Average } \\
\text { SD/Avg }\end{array}$ & $\begin{array}{l}0.60 \\
0.02\end{array}$ & $\begin{array}{l}2.24 \\
0.06\end{array}$ & $\begin{array}{l}0.26 \\
0.30\end{array}$ & $\begin{array}{l}1.47 \\
0.13\end{array}$ & $\begin{array}{l}1.84 \\
0.07\end{array}$ & $\begin{array}{l}1.14 \\
0.41\end{array}$ & $\begin{array}{l}1.08 \\
0.39\end{array}$ & $\begin{array}{l}1.13 \\
0.40\end{array}$ & $\begin{array}{l}3.14 \\
0.13\end{array}$ & $\begin{array}{l}1.80 \\
0.13\end{array}$ & $\begin{array}{l}3.29 \\
0.08\end{array}$ & $\begin{array}{l}3.40 \\
0.09\end{array}$ & $\begin{array}{l}2.25 \\
0.17\end{array}$ & $\begin{array}{l}0.52 \\
0.18\end{array}$ \\
\hline & \multirow{2}{*}{$\begin{array}{l}\text { ORDER } \\
\text { INFINITY }\end{array}$} & Rho1 $=0.1$ Rho2 $=-0.8$ & $\begin{array}{l}\text { Averege } \\
\text { SD/Avg }\end{array}$ & $\begin{array}{l}0.79 \\
0.02\end{array}$ & $\begin{array}{l}2.30 \\
0.10\end{array}$ & $\begin{array}{l}0.14 \\
0.51\end{array}$ & $\begin{array}{l}0.39 \\
0.13\end{array}$ & $\begin{array}{l}2.18 \\
0.15\end{array}$ & $\begin{array}{l}0.93 \\
0.21\end{array}$ & $\begin{array}{l}0.83 \\
0.24\end{array}$ & $\begin{array}{l}0.84 \\
0.25\end{array}$ & $\begin{array}{l}431 \\
0.18\end{array}$ & $\begin{array}{l}2.14 \\
0.18\end{array}$ & $\begin{array}{l}2.92 \\
0.13\end{array}$ & $\begin{array}{l}3.89 \\
0.15\end{array}$ & $\begin{array}{l}0.46 \\
0.16\end{array}$ & $\begin{array}{l}0.60 \\
0.11\end{array}$ \\
\hline & & Rhof $=0.7$ Rho2 $=-0.2$ & $\begin{array}{l}\text { Average } \\
\text { SDravg }\end{array}$ & $\begin{array}{l}0.81 \\
0.03\end{array}$ & $\begin{array}{l}2.68 \\
0.09\end{array}$ & $\begin{array}{l}0.74 \\
0.26\end{array}$ & $\begin{array}{l}0.52 \\
0.25 \\
\end{array}$ & $\begin{array}{l}2.44 \\
0.10\end{array}$ & $\begin{array}{l}0.87 \\
0.31\end{array}$ & $\begin{array}{l}0.77 \\
0.34\end{array}$ & $\begin{array}{l}0.79 \\
0.35\end{array}$ & $\begin{array}{l}5.28 \\
0.20\end{array}$ & $\begin{array}{l}2.47 \\
0.20\end{array}$ & $\begin{array}{l}2.62 \\
0.14\end{array}$ & $\begin{array}{l}3.94 \\
0.14\end{array}$ & $\begin{array}{l}0.55 \\
0.21\end{array}$ & o. \\
\hline
\end{tabular}

\begin{tabular}{|c|c|c|c|c|c|c|c|c|c|c|c|c|c|c|c|c|c|}
\hline \multirow{8}{*}{ 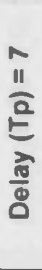 } & \multirow{3}{*}{ ORDER 1} & Rho1 $=0.1$ Rho2 $=-0.8$ & $\begin{array}{l}\text { Average } \\
\text { SDiAvg }\end{array}$ & $\begin{array}{l}0.82 \\
0.02\end{array}$ & $\begin{array}{l}2.05 \\
0.11\end{array}$ & $\begin{array}{l}2.42 \\
0.13\end{array}$ & $\begin{array}{l}2.71 \\
0.13\end{array}$ & $\begin{array}{l}1.71 \\
0.18\end{array}$ & $\begin{array}{l}1.22 \\
0.25\end{array}$ & $\begin{array}{l}9.18 \\
0.24\end{array}$ & $\begin{array}{l}1.21 \\
0.25\end{array}$ & $\begin{array}{l}3.07 \\
0.21\end{array}$ & $\begin{array}{l}1.66 \\
0.99\end{array}$ & $\begin{array}{l}2.69 \\
0.12\end{array}$ & $\begin{array}{l}3.60 \\
0.15\end{array}$ & $\begin{array}{l}0.54 \\
0.11\end{array}$ & $\begin{array}{l}0.57 \\
0.09\end{array}$ \\
\hline & & & & & & & & & & & & & & & & & \\
\hline & & Rho1-0.7 Rho2 $=0.2$ & $\begin{array}{l}\text { Average } \\
\text { SDrAyo }\end{array}$ & 0.87 & 3.00 & $\begin{array}{l}1.15 \\
0.36\end{array}$ & $\begin{array}{l}2.57 \\
0.09\end{array} \mid$ & 273 & 1.35 & 1.28 & $\begin{array}{c}1.30 \\
0.30\end{array}$ & 8.29 & 2.53 & 3.44 & 6.11 & 0.44 & 068 \\
\hline & & & & & & & & & & & & & & & & & 0.14 \\
\hline & \multirow{4}{*}{$\begin{array}{l}\text { ORDER } \\
\text { INFINITY }\end{array}$} & Rho1 $=0.1$ Rho $=-08$ & Average & 0.85 & 2.63 & 0.28 & 3.18 & 2.75 & 1.33 & 1.19 & 1.20 & 7.48 & 310 & 282 & 411 & 050 & 0.82 \\
\hline & & Rho $=0.1$ Khoz -0.8 & SO/Avg & 002 & 0.12 & 0.51 & 0.14 & 0.18 & 0.24 & 0.27 & 0.27 & 0.23 & 024 & 0.17 & 0.18 & 0.12 & 0.09 \\
\hline & & & Averago & & 3.29 & 0.46 & 248 & 3.11 & 104 & 0.98 & 0.98 & 32.86 & 4.14 & 3.11 & 508 & 046 & \\
\hline & & Rho $1=0.7$ Rho2 $=-0.2$ & SD/Avg & 0.02 & 0.11 & 0.28 & 0.13 & 0.11 & 0.34 & 0.34 & 0.34 & 0.20 & 0.28 & 0.15 & 0.17 & 0.12 & 0.10 \\
\hline
\end{tabular}

\begin{tabular}{|c|c|c|c|c|c|c|c|c|c|c|c|c|c|c|c|c|}
\hline \multirow{3}{*}{ ORDER 1} & Rho1 $=0.1$ Rho2 $=-0.8$ & $\begin{array}{l}\text { Averago } \\
\text { SOIAvg }\end{array}$ & $\begin{array}{l}0.90 \\
0.02\end{array}$ & $\begin{array}{l}2.74 \\
0.14\end{array}$ & $\begin{array}{l}3.20 \\
0.19\end{array}$ & $\begin{array}{l}.14 \\
0.13 \\
\end{array}$ & $\begin{array}{l}2.94 \\
0.19\end{array}$ & $\begin{array}{l}1.65 \\
0.29\end{array}$ & $\begin{array}{l}1.62 \\
0.28\end{array}$ & $\begin{array}{l}1.65 \\
0.28\end{array}$ & $\begin{array}{l}6.27 \\
0.28\end{array}$ & $\begin{array}{l}3.21 \\
0.28\end{array}$ & $\begin{array}{l}2.66 \\
0.12\end{array}$ & $\begin{array}{l}5.16 \\
0.19\end{array}$ & $\begin{array}{l}0.81 \\
0.07\end{array}$ & $\begin{array}{l}0.84 \\
0.08\end{array}$ \\
\hline & & & & & & & & & & & & & & & & \\
\hline & $R h o 1=0.7$ Rho2 $=-0.2$ & Average & 0.97 & 3.48 & 3.21 & 2.59 & 3.30 & 1.24 & 1.19 & 1.19 & 1687 & 4.65 & 3.02 & 7.66 & 0.57 & 0.78 \\
\hline & & & & & & & & & & & & & & & & \\
\hline \multirow{4}{*}{$\begin{array}{l}\text { ORDER } \\
\text { INFINITY }\end{array}$} & Rho1 $=0,1$ Rho $=-0.8$ & Ave & 090 & 3.18 & 9.49 & 3.39 & 3.88 & 1.63 & 1.55 & 9.56 & 11.26 & 5.83 & 248 & 4.71 & 061 & 06 \\
\hline & 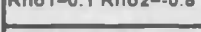 & SO/Avg & 0.02 & 0.17 & 0.43 & 0.17 & 0.21 & 0.22 & 0.23 & 0.23 & 0.31 & 0.31 & 0.18 & 0.24 & 0.12 & 0.12 \\
\hline & & & & & & & & & & & & & & & & \\
\hline & Rho1 $=0.7$ Rho $2=-0.2$ & $\begin{array}{l}\text { Average } \\
\text { SD/Avg }\end{array}$ & $\begin{array}{l}0.80 \\
0.02\end{array}$ & $\begin{array}{l}3.79 \\
0.15\end{array}$ & $\begin{array}{l}0.80 \\
0.47\end{array}$ & $\begin{array}{l}2.72 \\
0.18\end{array}$ & $\begin{array}{l}385 \\
0.15\end{array}$ & $\begin{array}{l}1.00 \\
0.29\end{array}$ & $\begin{array}{l}0.96 \\
0.27\end{array}$ & $\begin{array}{l}0.94 \\
0.29\end{array}$ & $\begin{array}{r}42.25 \\
0.32\end{array}$ & $\begin{array}{l}8.13 \\
0.33\end{array}$ & $\begin{array}{l}2.70 \\
0.15\end{array}$ & $\begin{array}{l}5.61 \\
0.20\end{array}$ & $\begin{array}{l}0.55 \\
0.14\end{array}$ & $\begin{array}{l}0.79 \\
0.10\end{array}$ \\
\hline
\end{tabular}

\section{Demand Process: MA (2)}

\begin{tabular}{|c|c|c|c|c|c|c|c|c|c|c|c|c|c|c|c|c|c|}
\hline & \multirow[b]{2}{*}{$\begin{array}{l}\text { Order of } \\
\text { Delay }\end{array}$} & \multirow[b]{2}{*}{ ma (2) Demand Parameters } & & \multicolumn{3}{|c|}{ 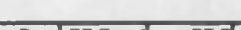 } & & & & \multirow{2}{*}{ AR AIC } & \multirow[b]{2}{*}{ ACF 7} & \multicolumn{5}{|c|}{ 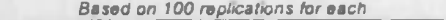 } \\
\hline & & & & $\begin{array}{l}\text { Rav } \\
\text { Time }\end{array}$ & TToral & FTT & FT2 29 & $\begin{array}{l}\text { FT 80\% } \\
\text { Enorgy }\end{array}$ & AR T & AR 2E & & & ACF 28 & CCF T & CCF 28 & $\begin{array}{l}\text { DWT Hase } \\
\text { (Level s) }\end{array}$ & $\begin{array}{l}\text { DWT Hast } \\
\text { (Level } \\
\text { Shannon }\end{array}$ \\
\hline \multirow{9}{*}{$\frac{m}{\frac{m}{2}}$} & \multirow{4}{*}{ ORDER 1} & Thet $1=0.7$ The ta $2=-0.2$ & $\begin{array}{l}\text { Average } \\
\text { SolAng }\end{array}$ & $\begin{array}{l}0.66 \\
0.02\end{array}$ & $\begin{array}{l}1.83 \\
0.07\end{array}$ & $\begin{array}{l}1.26 \\
0.14\end{array}$ & $\begin{array}{l}223 \\
0.08\end{array}$ & $\begin{array}{l}1.67 \\
0.11\end{array}$ & $\begin{array}{l}1.05 \\
0.17\end{array}$ & $\begin{array}{l}.05 \\
0.17\end{array}$ & $\begin{array}{l}05 \\
0.17\end{array}$ & $\begin{array}{l}2.10 \\
0.12\end{array}$ & $\begin{array}{l}1.31 \\
0.10\end{array}$ & $\begin{array}{l}2.38 \\
0.10\end{array}$ & $\begin{array}{l}2.42 \\
0.10\end{array}$ & $\begin{array}{l}0.49 \\
0.09\end{array}$ & $\begin{array}{l}0.53 \\
0.06\end{array}$ \\
\hline & & \multirow{3}{*}{ Theta $1=0.1$ Thete $2=-0.8$} & & & & & & & & & & & & & & & \\
\hline & & & Averago & 0.73 & 300 & 0.24 & 2.12 & 305 & 1.12 & 1.05 & 105 & 465 & 2.12 & 5.72 & 5.89 & 0.33 & 0.46 \\
\hline & & & SDIAvg & 0.01 & 0.05 & 0.43 & 0.12 & 0.07 & 0.27 & 0.24 & 024 & 0.12 & 0.12 & 0.08 & 0.08 & 0.12 & 0.14 \\
\hline & & & & & & & & & & & & & & & & & \\
\hline & \multirow{4}{*}{$\begin{array}{l}\text { ORDER } \\
\text { INFINITY }\end{array}$} & \multirow{2}{*}{ Theta $1=0.7$ Thera $2=-0.2$} & Averago & 0.77 & 257 & 0.11 & 0.38 & 2.84 & 0.99 & 0.90 & 0.91 & 475 & 2.17 & 2.87 & 3.87 & 0.44 & 0.83 \\
\hline & & & SO/Avg & 0.02 & 0.09 & 0.58 & 0.14 & 0.13 & 0.16 & 0.16 & 0.17 & 0.18 & 0.19 & 0.14 & 0.16 & 0.15 & 0.03 \\
\hline & & \multirow{2}{*}{ Thete $1=0.1$ Theta $2=-0.8$} & & & & & & & & & & & & & & & \\
\hline & & & Averago & 0.81 & 3.13 & $\begin{array}{l}0.15 \\
0.75\end{array}$ & 0.37 & $\begin{array}{ll}3.13 \\
0.99\end{array}$ & 0.96 & 0.89 & 089 & 6.13 & 269 & 3.41 & 5.02 & 0.41 & $\begin{array}{l}0.61 \\
0.06\end{array}$ \\
\hline & & & & & & & & & & & & & & & & & \\
\hline \multirow{10}{*}{$\frac{\hat{a}}{\frac{\hat{a}}{2}}$} & \multirow{5}{*}{ ORDER 1} & \multirow{3}{*}{ Thele $1=0.7$ Theta $2=-0.2$} & Averege & 0.81 & 2.74 & 265 & 334 & 3.28 & 180 & 1.74 & 180 & 495 & 2.15 & 332 & 485 & 060 & 0.65 \\
\hline & & & SDIAvg & 002 & 0.10 & 0.16 & 0.11 & 0.13 & 0.28 & 026 & 028 & 019 & 0.18 & 0.12 & 0.15 & 0.10 & 0.08 \\
\hline & & & & & & & & & & & & & & & & & \\
\hline & & \multirow{2}{*}{ Theta $1=0.1$ Thets $2=-0.8$} & Average & 0.89 & 357 & 1.49 & 3.02 & 3.57 & 1.77 & 1.64 & 1.65 & 1000 & 286 & 4.33 & 7.80 & 0.49 & 0.60 \\
\hline & & & SO/Avg & 0.01 & 0.08 & 0.30 & 0.09 & 0.08 & 0.25 & 0.24 & 0.25 & 0.19 & 0.17 & 0.10 & 0.12 & 0.09 & 0.13 \\
\hline & \multirow{5}{*}{$\begin{array}{l}\text { ORDER } \\
\text { INFINITY }\end{array}$} & \multirow{3}{*}{ Thet 10.7 Theta $2=-0.2$} & & & & & & & & & & & & & & & \\
\hline & & & Average & 0.85 & 3.18 & 0.21 & 3.44 & 4.46 & 1.77 & 1.43 & 1.44 & 1227 & 3.63 & 3.19 & 4.90 & 0.48 & 0.68 \\
\hline & & & SO/Avg & 0.02 & 0.11 & 0.55 & 0.13 & 0.14 & 0.24 & 0.21 & 0.21 & 0.21 & 0.25 & 0.17 & 0.18 & 0.13 & 0.03 \\
\hline & & \multirow[b]{2}{*}{ Thete $1=0.1$ There $2=-0.8$} & & & 370 & & 279 & 378 & 154 & 146 & 148 & 3925 & 344 & 356 & 5001 & 046 & 063 \\
\hline & & & $\begin{array}{l}\text { Average } \\
\text { SDIAvg }\end{array}$ & 0.02 & 0.11 & 0.43 & 0.13 & 0.11 & 0.22 & 023 & 022 & $\begin{array}{r}38.25 \\
021\end{array}$ & 0.28 & 0.18 & 0.17 & 0.13 & $\begin{array}{l}0.3 \\
0.15\end{array}$ \\
\hline & & & & & & & & & & & & & & & & & \\
\hline \multirow{10}{*}{$\frac{\frac{\pi}{2}}{\frac{\pi}{2}}$} & & & Averago & 0.91 & 340 & 3.70 & 343 & 5.24 & 2.57 & 2.4 & 257 & 994 & 398 & 3.08 & 8.66 & 0.85 & 0.72 \\
\hline & & Thetais $=0.7$ Theta $2=-0.2$ & SOIAvg & 0.02 & 0.13 & 0.98 & 0.12 & 0.18 & 0.20 & 0.17 & 0.18 & 0.25 & 0.25 & 0.12 & 0.18 & 0.08 & 0.05 \\
\hline & ORDER 1 & & & & & & & & & & & & & & & & \\
\hline & & Then $1=0,1$ Thet $2=0.8$ & Averago & 0.98 & 301 & 352 & 2.87 & 3.94 & 239 & 2.23 & 222 & 19.54 & 501 & 3.43 & 8.83 & 0.80 & 0.87 \\
\hline & & Therais $=0.1$ Thete $2=-0.8$ & SO/Ang & 0.01 & 011 & 0.17 & 0.11 & 0.12 & 0.23 & 0.26 & 0.24 & 0.25 & 0.25 & 0.11 & 0.16 & 007 & 0.14 \\
\hline & & & & & & & & & & & & & & & & & \\
\hline & & Theta $=0.7$ Theta $=0.2$ & Average & 091 & 3.79 & 139 & 355 & 8.63 & 2.39 & 2.02 & 203 & 19.32 & 7.18 & 2.79 & 5.57 & 061 & 0.72 \\
\hline & ORDER & & SO/Avg & 0.02 & 0.16 & 0.45 & 0.16 & 0.21 & 0.18 & 0.17 & 0.17 & 0.31 & 0.31 & 0.17 & 0.23 & 0.12 & 0.04 \\
\hline & INFINITY & & Avorago & 0.96 & 4.14 & 0.4 & 2.93 & 422 & 2.06 & 1.87 & 1.85 & 5009 & 8.61 & 294 & 6.17 & 0.57 & 070 \\
\hline & & Theta $1=0.1$ Theta $2=-0.8$ & SD/Avg & 0.01 & 0.15 & 0.51 & 0.15 & 0.15 & 0.21 & 0.23 & 0.22 & 0.33 & 033 & 0.15 & 0.21 & 0.13 & 0.19 \\
\hline
\end{tabular}

Raw Time Based on data in the time domain
FT Total, FT 7, FT 28 : Based on amplitudes of total, firt 7 and firs 28 frequencies ather fourier traneform

FT $80 \%$ Energy Based on ampitudes of first as many frequencies as have 80\% of the enengy in the data after fourier tranatorm

AR 7. AR 28: Based on autoregressive parameters (AR) d time series represented as AR modeis of order 7 and 28

NR AC: Bssed on autoregressive paramoters (AR) of time sertics represented as AR models of order defined by minumiang Akaike information criteria (ANC)

CCF 7 CCF 28 Bamed

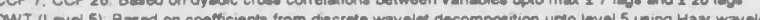

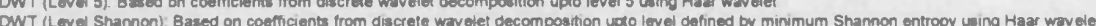




\section{Appendix F}

Average and coefficient of variation of rogue seasonality index values for a 3 echelon MTS system based on different feature parameter combinations; Only order and inventory variables used in the index computation

\section{Demand Process: MA (1)}

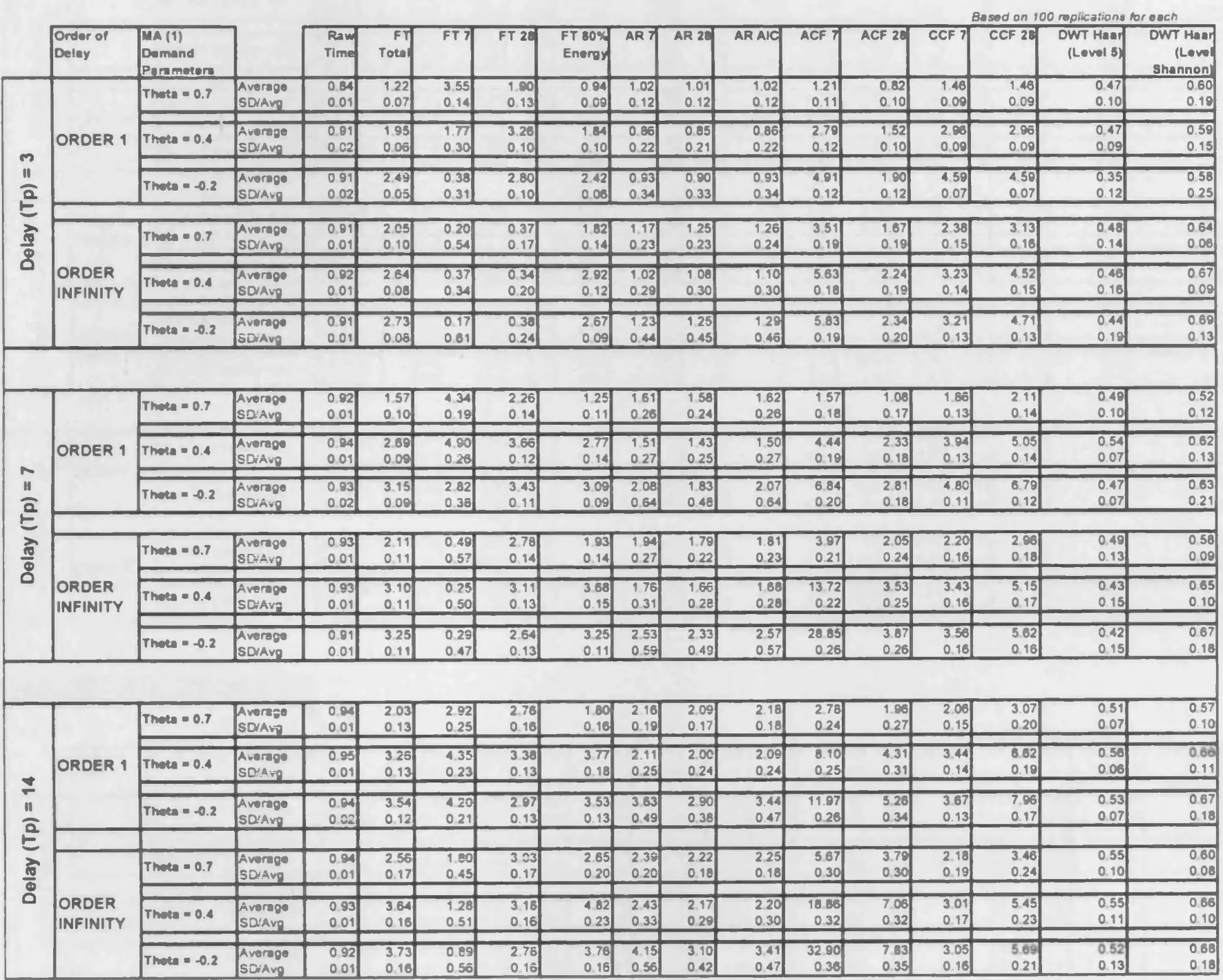

\section{Demand Process: Gaussian random}

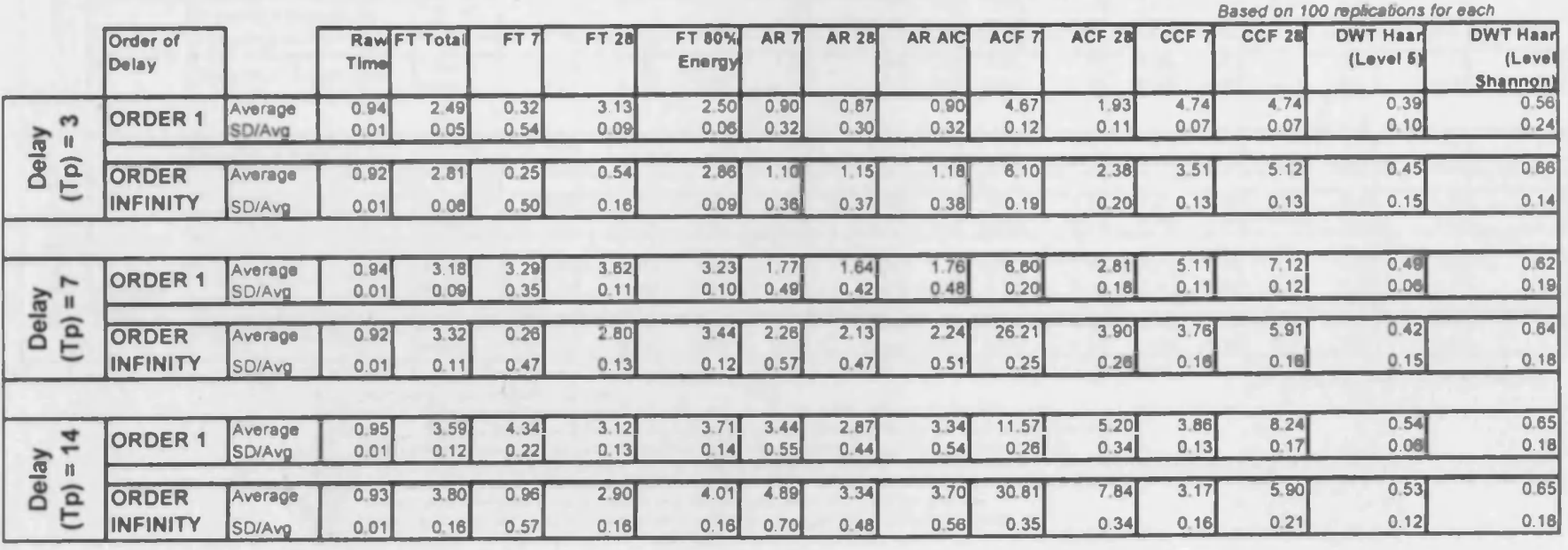




\section{Appendix F Continued}

\section{Demand Process: AR (2)}

\begin{tabular}{|c|c|c|c|c|c|c|c|c|c|c|c|c|c|c|c|c|c|}
\hline & \multirow[b]{2}{*}{$\begin{array}{l}\text { Order of } \\
\text { Delay }\end{array}$} & \multirow[b]{2}{*}{$\begin{array}{l}\text { AR (2) Demand } \\
\text { Parnmoters }\end{array}$} & & \multirow[b]{2}{*}{$\begin{array}{l}\text { Raw } \\
\text { Time }\end{array}$} & \multirow[b]{2}{*}{ FT Total } & \multirow[b]{2}{*}{ FT7 } & \multirow[b]{2}{*}{ FT 2E } & \multirow[b]{2}{*}{$\begin{array}{l}\text { FT } 80 \% \\
\text { Eneroy }\end{array}$} & \multirow[b]{2}{*}{ AR T } & \multirow{2}{*}{\multicolumn{2}{|c|}{ AR 2EAR AIC }} & \multirow[b]{2}{*}{ ACF 7} & \multirow[b]{2}{*}{ ACF 28 } & \multicolumn{4}{|c|}{ Based on 100 replications for each } \\
\hline & & & & & & & & & & & AR AIC & & & CCF 7 & CCF 25 & $\begin{array}{l}\text { DWT Haaj } \\
\text { (Lovel 5) }\end{array}$ & 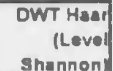 \\
\hline \multirow{7}{*}{ 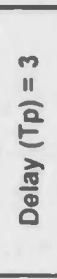 } & \multirow{3}{*}{ ORDER 1} & Rho1 $=0.1$ Rho $2=-0.8$ & $\begin{array}{l}\text { Average } \\
\text { SDiAvg }\end{array}$ & $\begin{array}{l}0.83 \\
0.02\end{array}$ & $\begin{array}{ll}1.08 \\
0.08\end{array}$ & $\begin{array}{ll}326 \\
0.14\end{array}$ & $\begin{array}{l}1.92 \\
0.14\end{array}$ & $\begin{array}{ll}0.62 \\
0.15\end{array}$ & $\begin{array}{l}0.81 \\
0.28\end{array}$ & $\begin{array}{l}0.78 \\
027\end{array}$ & $\begin{array}{l}0.87 \\
027\end{array}$ & $\begin{array}{l}1.10 \\
0.15\end{array}$ & $\begin{array}{ll}0.81 \\
0.13\end{array}$ & $\begin{array}{r}1.63 \\
0.09\end{array}$ & $\begin{array}{l}1.63 \\
0.09\end{array}$ & $\begin{array}{lll}0 & 46 \\
0 & 10\end{array}$ & $\begin{array}{l}0.58 \\
0.29\end{array}$ \\
\hline & & & & & & & & & & & & & & & & & \\
\hline & & $R$ ho1 $=0.7$ Rho $2=-0.2$ & $\begin{array}{l}\text { average } \\
\text { soliAvg }\end{array}$ & $\begin{array}{l}0.81 \\
0.02\end{array}$ & $\begin{array}{l}2.27 \\
0.06\end{array}$ & $\begin{array}{l}0.33 \\
0.30 \\
0\end{array}$ & $\begin{array}{l}1.04 \\
0.13\end{array}$ & $\begin{array}{l}1.99 \\
0.07\end{array}$ & $\begin{array}{l}1.15 \\
0.46\end{array}$ & $\begin{array}{l}1.09 \\
0.43\end{array}$ & $\begin{array}{l}1.14 \\
0.45 \\
\end{array}$ & $\begin{array}{l}4.97 \\
0.12\end{array}$ & $\begin{array}{l}1.72 \\
0.14\end{array}$ & $\begin{array}{l}3.24 \\
0.08\end{array}$ & $\begin{array}{l}3.24 \\
0.08\end{array}$ & $\begin{array}{l}0.28 \\
0.18\end{array}$ & $\begin{array}{l}0.59 \\
0.18\end{array}$ \\
\hline & & & & & & & & & & & & & & & & & \\
\hline & \multirow{3}{*}{$\begin{array}{l}\text { ORDER } \\
\text { INFINITY }\end{array}$} & Rho1 $=0.1$ Rho $2=-0.8$ & Avorage & 0.92 & 2.03 & 0.17 & 0.39 & 179 & 1.02 & 1.03 & 1.05 & 3.64 & 1.85 & 249 & 3.24 & 0.44 & 0.60 \\
\hline & & & 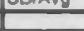 & 0.0 & & 0.40 & 0.12 & & & & & 0.010 & 皮 & & 0.95 & & 012 \\
\hline & & RnO1 $=0.7$ Rho 2 $=0.2$ & Average & 0.85 & 2.40 & 0.79 & 0.55 & 2.17 & 1.34 & 1.75 & 1.20 & 485 & 2.19 & 234 & 3.49 & 0.54 & 0.71 \\
\hline & & & & & & & & & & & & & & & & & \\
\hline \multirow{9}{*}{$\begin{array}{l}\frac{n}{2} \\
\frac{\pi}{2} \\
\frac{\pi}{0}\end{array}$} & \multirow{4}{*}{ ORDER 1} & & & & & & & & & & & 295 & & & 275 & & 0,5 \\
\hline & & Rho1 $=0.1$ Rho $2=0.8$ & SO/Avg & $\begin{array}{l}0.01 \\
\end{array}$ & 0.11 & 0.17 & 0.14 & 0.18 & 0.24 & 0.23 & 0.24 & 0.21 & 0.18 & 0.14 & 0.15 & 0.08 & 0.15 \\
\hline & & & $A_{v}$ & 0.87 & 2.90 & 1.86 & 2.00 & 287 & 1.44 & 1.32 & 1.42 & 6.92 & 2.70 & 3.70 & 5.5र्श & 0.41 & 0.05 \\
\hline & & Rho1 $=0.7$ Rho2 $=-0.2$ & SOIAvo & 0.02 & 0.08 & 0.46 & 0.11 & 0.09 & 0.39 & 0.30 & 0.30 & 0.20 & 0.17 & 0.11 & 0.12 & 0.08 & 0.17 \\
\hline & & & & & & & & & & & & & & & & & \\
\hline & \multirow{4}{*}{$\begin{array}{l}\text { ORDER } \\
\text { INFINITY }\end{array}$} & $R h 01=0.1 R h 02=-0.8$ & Average & 0.03 & 2.33 & 0.35 & 2.82 & 2.14 & 1.46 & 1.39 & 141 & 5.77 & 2.67 & 2.63 & 3.61 & 0.46 & 0.57 \\
\hline & & & SOIAVIS & 0.01 & 0.12 & 0.63 & 0.14 & 0.18 & 0.27 & 0.27. & 0.27 & 0.24 & 0.23 & 0.16 & 0.18 & 0.14 & 0.12 \\
\hline & & Rhol=0.7 Rho2=-0.2 & Average & 0.88 & 2.99 & 0.47 & 2.26 & 2.81 & 1.23 & 1.13 & 1.17 & 32.94 & 3.74 & 2.97 & 4.76 & 0.42 & 0.70 \\
\hline & & & SD/AVg & 0.02 & 0.11 & 0.24 & 0.14 & 0.12 & 0.39 & 0.36 & 0.37 & 0.28 & 0.27 & 0.16 & 0.16 & 0.14 & 0.12 \\
\hline \multirow{9}{*}{$\begin{array}{l}\frac{+}{2} \\
\frac{11}{2} \\
\frac{\pi}{0} \\
\frac{\pi}{0}\end{array}$} & \multirow{4}{*}{ ORDER 1} & & Average & 0.95 & 2.34 & 3.51 & 3.11 & 1.94 & 1.53 & 1.48 & 1.59 & 4.15 & 2.86 & 2.51 & 4.10 & 0.54 & 0.57 \\
\hline & & Rhot $=0.1$ Rho $=-0.8$ & SO/Avg & 0.01 & 0.14 & 0.24 & 0.15 & 0.18 & 0.21 & 0.21 & 0.21 & 0.26 & 0.27 & 5 & 0.20 & 0.06 & 0.08 \\
\hline & & Rhn =0 7 Phn $=0$ & & & 3.30 & 3.81 & 261 & 3.13 & 1.18 & 1.10 & 173 & 12.21 & 5.23 & 3.13 & 6.95 & 050 & 0.71 \\
\hline & & $R$ Ro $=0.7 R_{0}=-0.2$ & SOit & 0.02 & 0.12 & 0.21 & 0.12 & 0.13 & 0.38 & 036 & 0.38 & 0.26 & 0.35 & 0.14 & 0.17 & 008 & 0.14 \\
\hline & & & & & & & & & & & & & & & & & \\
\hline & \multirow{4}{*}{$\begin{array}{l}\text { ORDER } \\
\text { INFINITY }\end{array}$} & $R h 01=0.1$ Rho $=-0.8$ & Average & 0.94 & 2.86 & 1.58 & 3.15 & 2.96 & 1.83 & 155 & 1.57 & 8.54 & 5.13 & 246 & 4.10 & 055 & 0.58 \\
\hline & & & SD/AVg & 0.01 & 0.17 & 0.47 & 0.17 & 0.21 & 0.21 & 0.21 & 0.21 & 0.31 & 0.31 & 0.19 & 0.24 & 0.14 & 0.13 \\
\hline & & & & & & & 250 & 336 & 1.03 & 0.96 & 0.97 & 3560 & 7.66 & 271 & 5.09 & 0.50 & 0.72 \\
\hline & & Rho1 $=0.7$ Rho $2=-0.2$ & SOIAvg & 0.02 & 0.16 & 0.49 & 0.16 & 0.15 & 0.33 & 0.31 & 0.33 & 0.38 & 0.36 & 0.16 & 0.21 & 0.14 & 0.11 \\
\hline
\end{tabular}

\section{Demand Process: MA (2)}

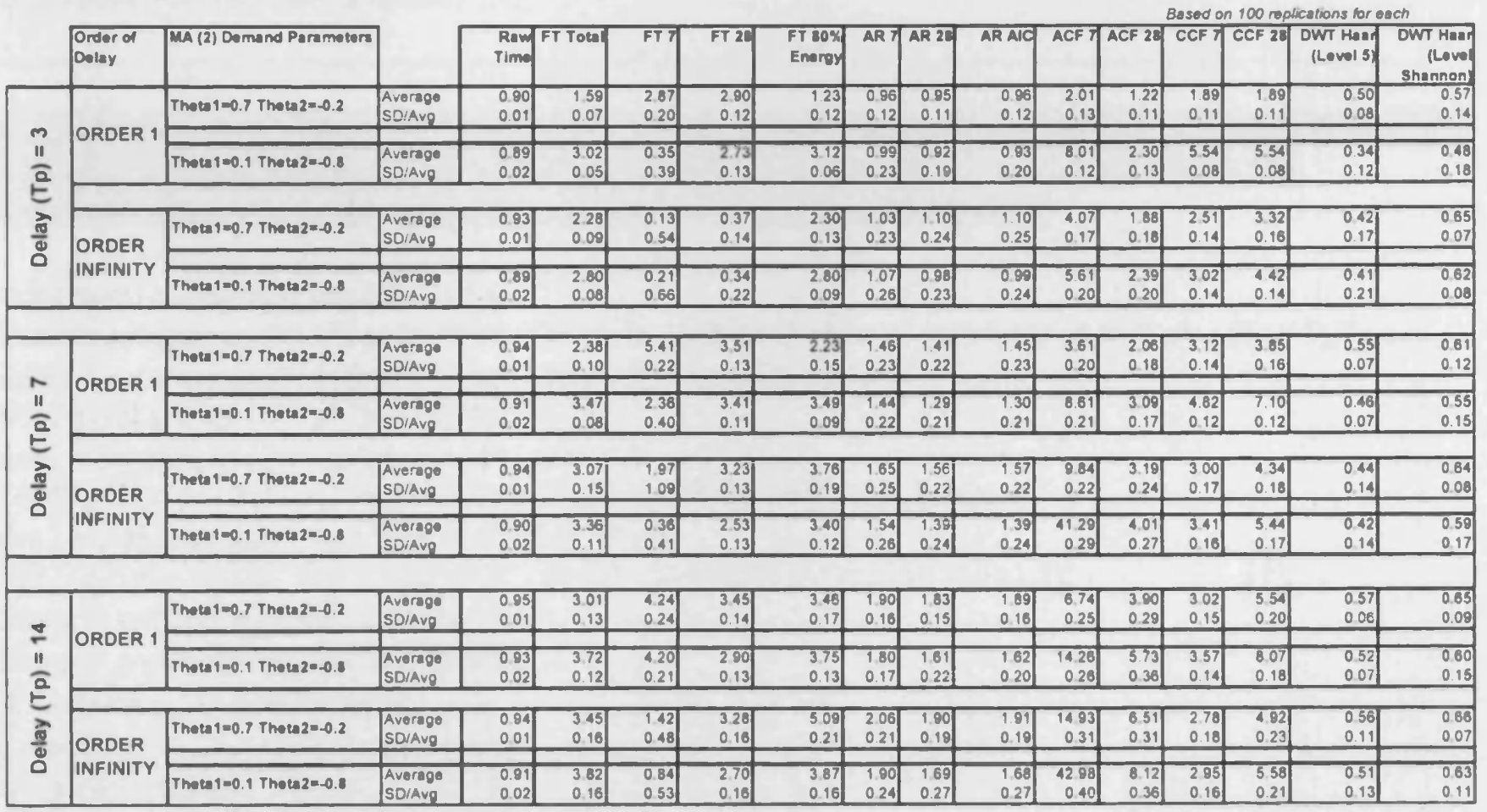

Rew Time Based on data in the time domain

FT TOtal, FT 7, FT 28 Based on amplitudes of total, first 7 and firex 28 trequencies ather fourier transtorm

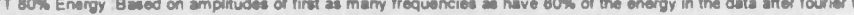

(A)

CWT (Level 5) Based on coefficierts form diacreto wavelef decompos upto $\max \pm 7$ lags and \pm 28 lages

DWT (Lever Shannon): Basod on coefficients from discrele wavelet decompostion upto level defined by mirimum Shannon entropy using Haar wavelef 


\section{Appendix G}

\section{Sample of Matlab code used for different tasks in Beer game system analysis}

\section{Analytical determination of critical points in frequency response as in Table 5.1 Example used: WIP / CONS for system with unoptimal parameters}

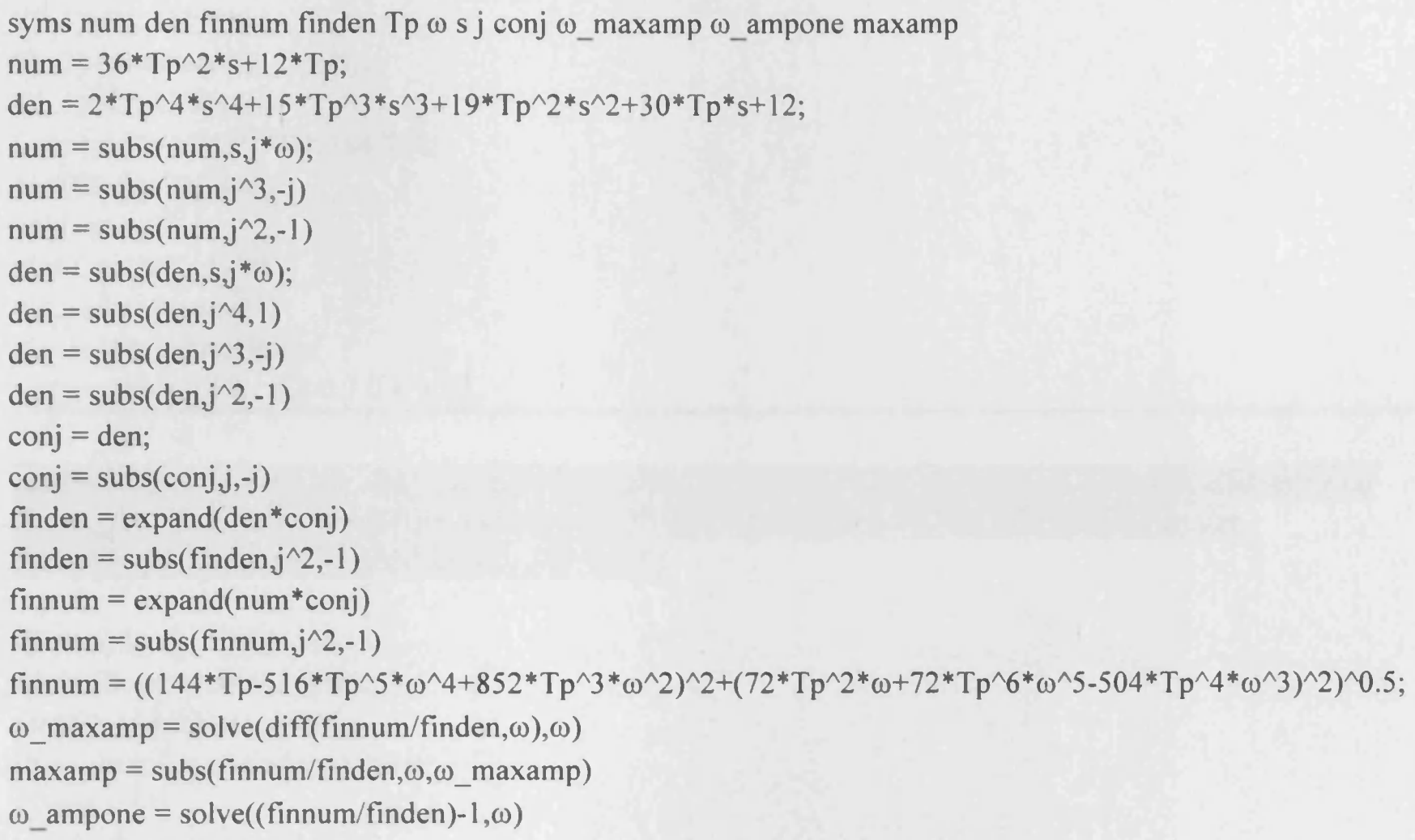

\section{Computation and plotting of frequency response for a four echelon system (as used in Figure 5.2) Example used: ORATE (Factory)/CONS for system with optimal parameters, LT 223 \\ Relevant single echelon transfer function given in Table 5.1 is utilised}

syms num 1 num 2 den 1 den2 Tp Tpl s

num $1=\operatorname{subs}\left(\operatorname{expand}\left(\left(14 * \mathrm{Tp}^{\wedge} 5^{*} \mathrm{~s}^{\wedge} 5+4 * T \mathrm{p}^{\wedge} 4^{*} \mathrm{~s}^{\wedge} 4-168^{*} \mathrm{Tp} \mathrm{p}^{\wedge} 3^{*} \mathrm{~s}^{\wedge} 3-48 * T \mathrm{Tp}^{\wedge} 2^{*} \mathrm{~s}^{\wedge} 2+2016 * \mathrm{Tp} * \mathrm{~s}+576\right)^{\wedge} 3\right), \mathrm{Tp}, 4\right)$;

num2 $=\operatorname{subs}\left(\operatorname{expand}\left(\left(14 * \mathrm{Tp}^{\wedge} 5^{*} \mathrm{~s}^{\wedge} 5+4 * \mathrm{Tp} 1^{\wedge} 4 * \mathrm{~s}^{\wedge} 4-168 * \mathrm{Tp} 1^{\wedge} 3 * \mathrm{~s}^{\wedge} 3-\right.\right.\right.$

$\left.\left.\left.48 * \mathrm{Tp}^{\wedge} 2 * \mathrm{~s}^{\wedge} 2+2016 * \mathrm{Tpl}{ }^{*} \mathrm{~s}+576\right)^{\wedge} 1\right), \mathrm{Tp} 1,3\right)$;

$\operatorname{den} 1=\operatorname{subs}\left(\operatorname{expand}\left(\left(8 * \mathrm{Tp}^{\wedge} 6 * \mathrm{~s}^{\wedge} 6+12 * \mathrm{Tp} \mathrm{p}^{\wedge} 5^{*} \mathrm{~s}^{\wedge} 5\right.\right.\right.$ -

$\left.\left.\left.140 * \mathrm{Tp}^{\wedge} 4 * \mathrm{~s}^{\wedge} 4+120^{*} \mathrm{Tp}^{\wedge} 3 \mathrm{~s}^{\wedge} \mathrm{s}^{\wedge} 3+672 * \mathrm{~T} \mathrm{p}^{\wedge} 2{ }^{*} \mathrm{~s}^{\wedge} 2+1440 * \mathrm{Tp} \mathrm{p}^{*} \mathrm{~s}+576\right)^{\wedge} 3\right), \mathrm{Tp}, 4\right) ;$

den $2=\operatorname{subs}\left(\operatorname{expand}\left(\left(8 * \mathrm{Tp} 1^{\wedge} 6^{*} \mathrm{~s}^{\wedge} 6+12 * \mathrm{Tp} 1^{\wedge} 5^{*} \mathrm{~s}^{\wedge} 5-\right.\right.\right.$

$\left.\left.\left.140 * \mathrm{Tpl}^{\wedge} 4{ }^{*} \mathrm{~s}^{\wedge} 4+120 * \mathrm{Tp} 1^{\wedge} 3 * \mathrm{~s}^{\wedge} 3+672 * \mathrm{Tp} 1^{\wedge} 2 * \mathrm{~s}^{\wedge} 2+1440 * \mathrm{Tp}{ }^{*} \mathrm{~s}+576\right)^{\wedge} 1\right), \mathrm{Tp} 1,3\right)$;

btemp $=\operatorname{sym} 2$ poly $($ expand $($ num $1 *$ num 2$))$;

atemp $=\operatorname{sym} 2$ poly $(\operatorname{expand}(\operatorname{den} 1 * \operatorname{den} 2))$;

if size(atemp,2)> size(btemp, 2)

$\mathrm{a}=\mathrm{atemp}$

$\mathrm{b}=\operatorname{zeros}(\operatorname{size}(\mathrm{a}))$;

$b(1+\operatorname{size}($ atemp, 2$)$-size (btemp,2):end $)=b$ temp $(1$ :end $)$;

else

$\mathrm{b}=\mathrm{btemp}$

$\mathrm{a}=\operatorname{zeros}(\operatorname{size}(\mathrm{b}))$;

a (1+size(btemp, 2)-size(atemp,2):end ) = atemp( 1 :end); 


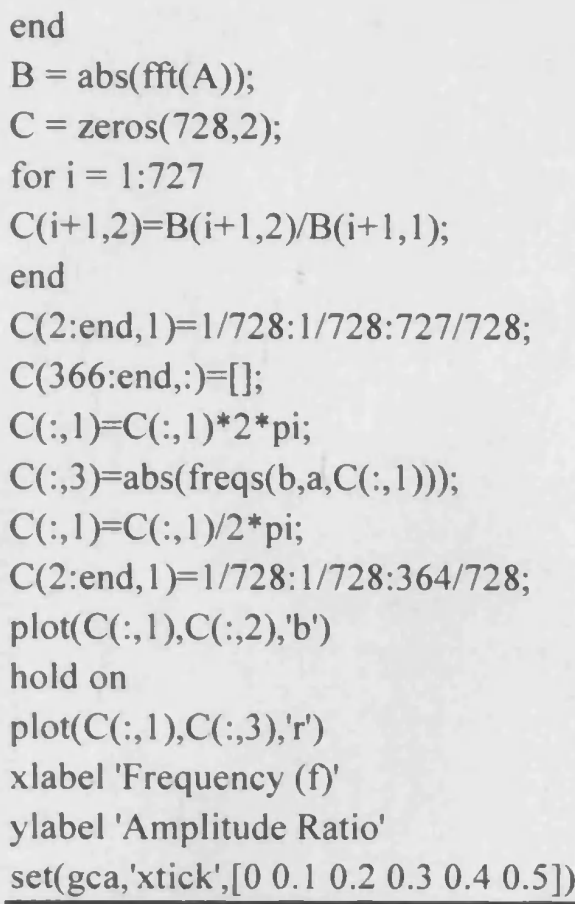

\section{Average frequency response (amplitude ratio) computation for various variables in simulated Beer game system; Computation across multiple sheets in multiple Excel workbooks Relevant for Figure 5.2, Appendices H.1, H.2}

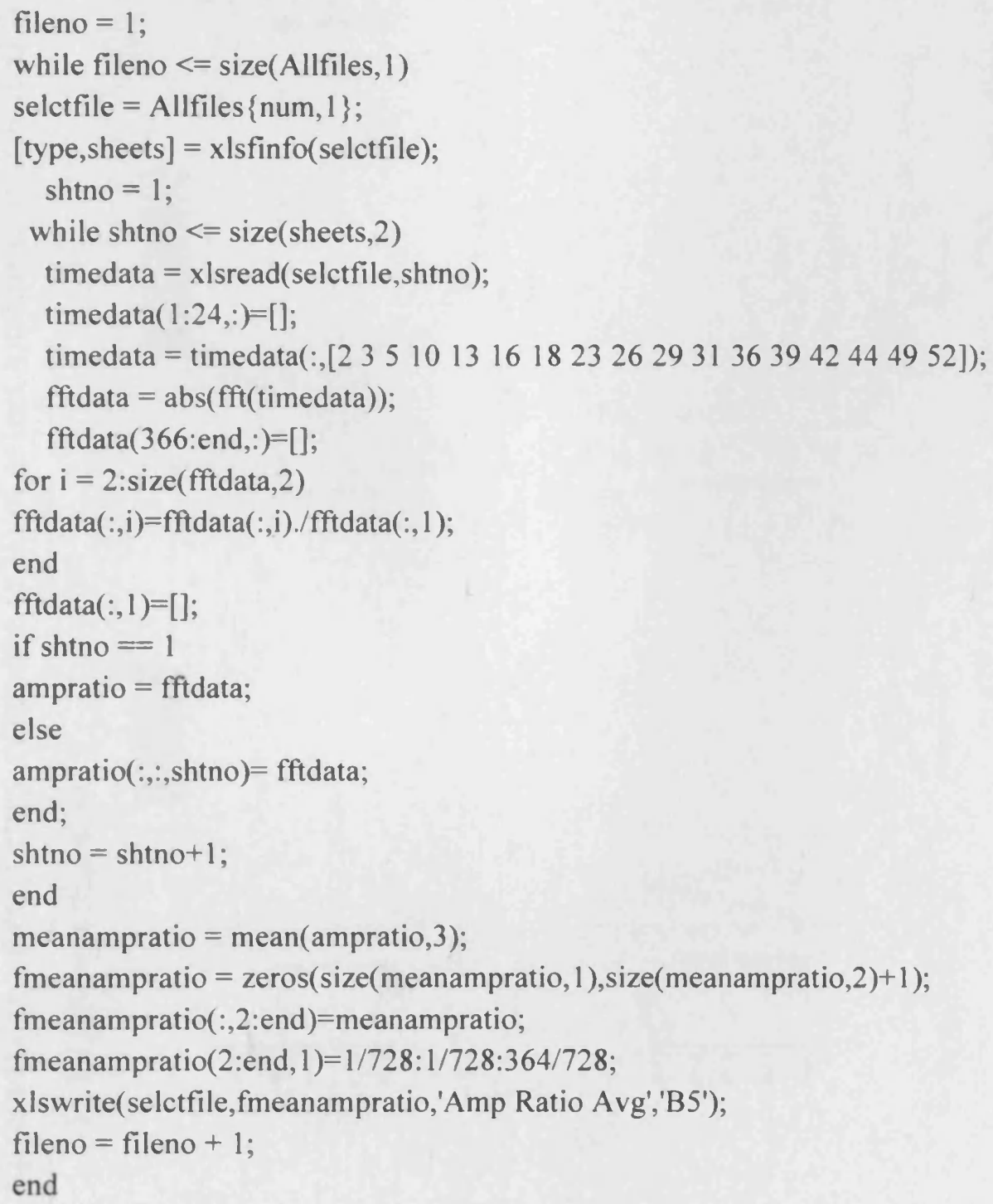


Appendix H - Frequency response (FR) of select variables computed from Beer game simulation outputs

H.1 Different lead times
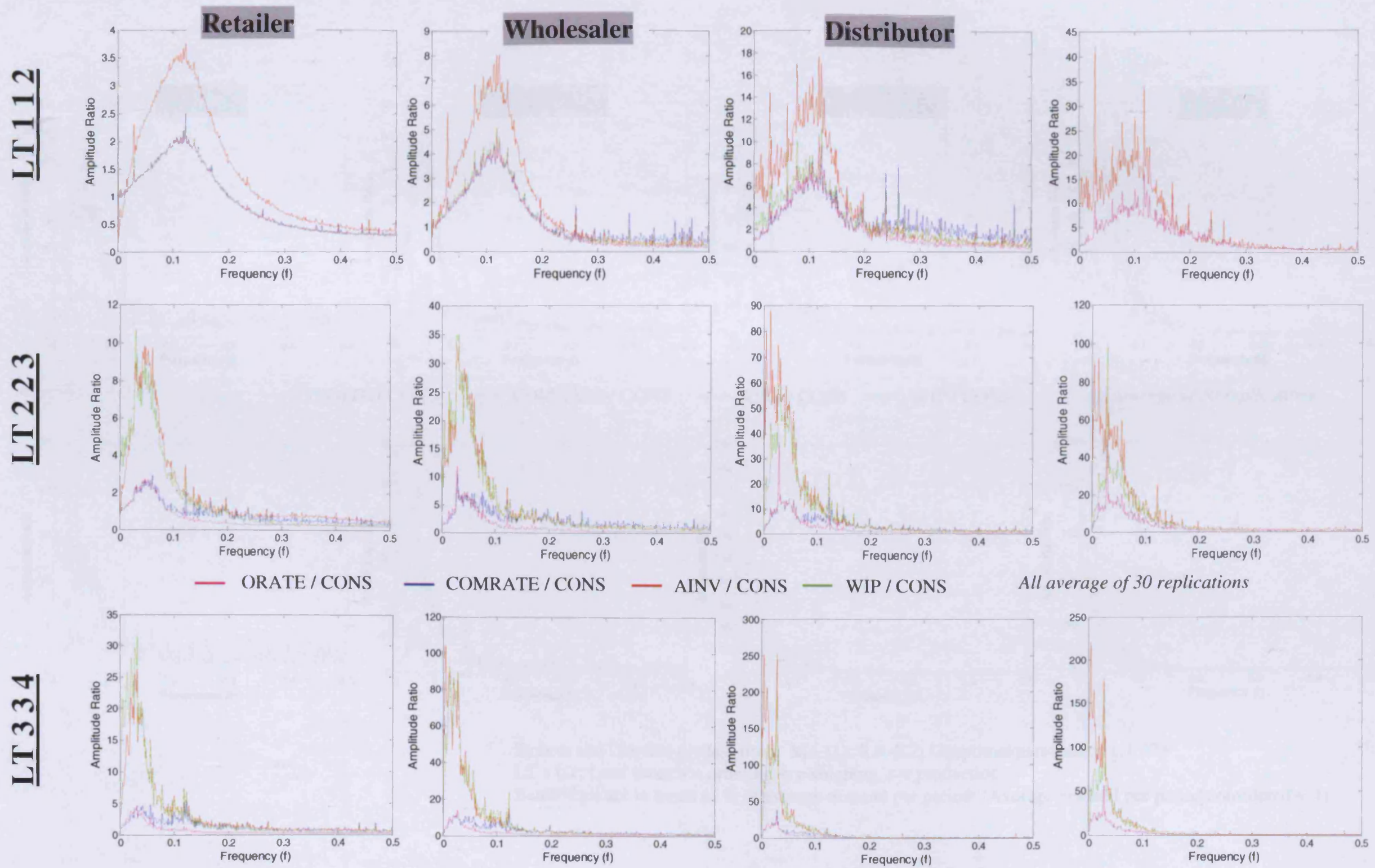

All average of 30 replications

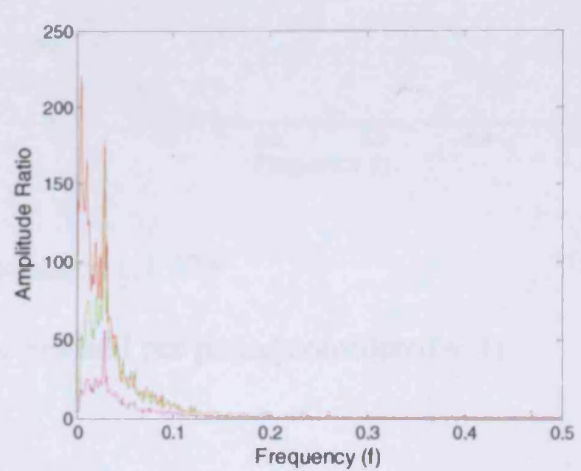




\section{H.2 Different batch sizes}
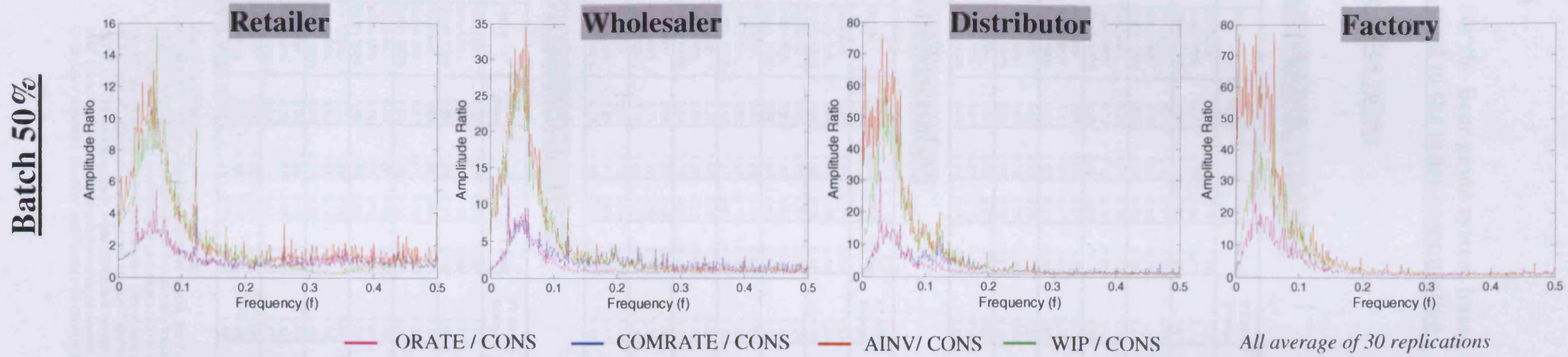

All average of 30 replications
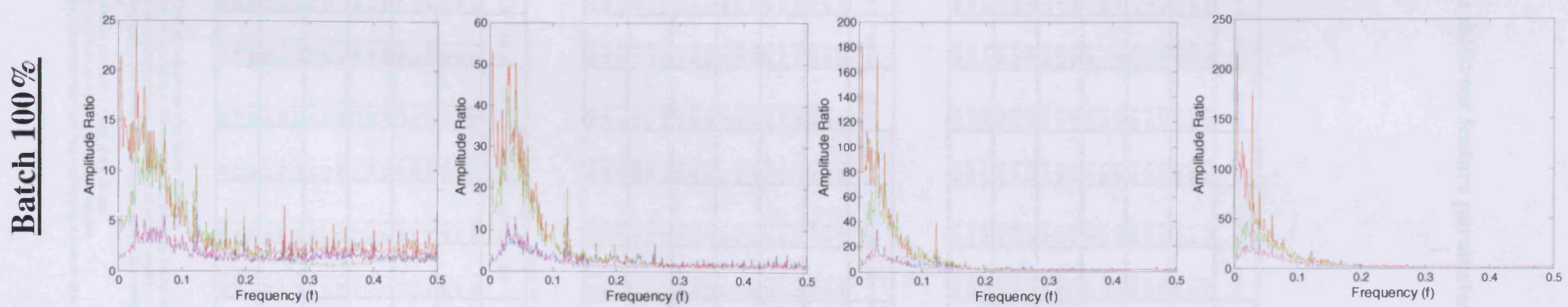

System and Demand process used: MA (1): $\theta=-0.2 ;$ Unoptimal parameters; LT 223

LT a b c: Lead times $\mathrm{a}=$ ordering, $\mathrm{b}=$ shipping, $\mathrm{c}=$ production

Batch sizes are in terms of $\%$ of average demand per period; (Average demand per period considered $=4$ ) 


\section{Appendix I}

Index values for the Beer game system based on different feature parameter combinations: All variables used in the index computation

\section{I.1 Average index values}

\section{Demand Process: AR(2) $\rho_{1}: 0.1 \rho_{1}:-0.8$}

\begin{tabular}{|c|c|c|c|c|c|c|c|c|c|c|c|c|c|c|c|c|}
\hline $\begin{array}{l}\text { Lead } \\
\text { Time }\end{array}$ & Ordering & Batch & $\begin{array}{l}\text { Raw } \\
\text { Time }\end{array}$ & FT Tolal & FT N & FT 28 & $\begin{array}{l}\text { FT } 80 \% \\
\text { Energy }\end{array}$ & AR 7 & AR 28 & AR AIC & ACF 7 & ACF 28 & $\operatorname{CCF} 7$ & CCF 28 & $\begin{array}{l}\text { DWT Hand } \\
\text { (Level 5) }\end{array}$ & $\begin{array}{l}\text { OWT Haar } \\
\text { (Level) } \\
\text { Shannon) }\end{array}$ \\
\hline \multirow{6}{*}{$\begin{array}{l}\text { ले } \\
\stackrel{5}{5}\end{array}$} & \multirow{3}{*}{ Unoptimal } & No Batch & 0.70 & 1.22 & 0.17 & 1.25 & 1.30 & 0.99 & 081 & 0.81 & 2.90 & 1.13 & 1.04 & 128 & 0.44 & 0.61 \\
\hline & & Batch $50 \%$ & 0.77 & 1.19 & 0.30 & 1.07 & 1.43 & 0.85 & 0.79 & 0.78 & 189 & 1.14 & 1.00 & 1.20 & 0.41 & \\
\hline & & Bateh $100 \%$ & 0.81 & 1.10 & 0.54 & 0.82 & 1.61 & 0.83 & 0.65 & 0.85 & 1.62 & 0.88 & 0.92 & 1.07 & 0.37 & \\
\hline & \multirow{3}{*}{ Optimal } & No Batch & 0.75 & 1.18 & 0.41 & 1.37 & 0.82 & 0.74 & 0.89 & 0.80 & 1.39 & 0.80 & 9.25 & 9.80 & 0.50 & 0.58 \\
\hline & & Batch $50 \%$ & 0.78 & 1.14 & 0.50 & 0.78 & 1.27 & 0.87 & 0.87 & 0.67 & 1.23 & 0.98 & 0.87 & 1.08 & 0.32 & 0.45 \\
\hline & & Batch $100 \%$ & 0.79 & 1.07 & 0.48 & 0.85 & 1.41 & 0.79 & 0.53 & 0.53 & 1.18 & 0.80 & 0.90 & 0.96 & 0.35 & \\
\hline \multirow{6}{*}{$\underset{\mathfrak{N}}{\mathfrak{n}}$} & \multirow{3}{*}{ Unoptimal } & No Batch & 0.72 & 1.21 & 0.13 & 1.19 & 1.05 & 0.00 & 0.81 & 0.82 & 198 & 9.19 & 1.22 & 1.55 & 0.44 & \\
\hline & & Batch $50 \%$ & 0.72 & 1.10 & 0.19 & 0.87 & 1.13 & 0.83 & 0.72 & 0.72 & 1.88 & 1.25 & 1.05 & 1.21 & 0.38 & \\
\hline & & Batch $100 \%$ & 0.73 & 0.95 & 0.39 & 0.79 & 1.20 & 0.75 & 0.60 & 0.60 & 1.31 & 0.89 & 0.83 & 0.90 & 0.34 & \\
\hline & \multirow{3}{*}{ Optimal } & No 8aich & 0.67 & 1.04 & 0.21 & 1.12 & 0.74 & 0.64 & 0.60 & 0.60 & 1.03 & 0.79 & 1.27 & 9.48 & 0.44 & \\
\hline & & Batch $50 \%$ & 0.72 & 1.07 & 0.41 & 0.69 & 0.09 & 0.77 & 0.58 & 0.58 & 1.28 & 1.21 & 1.04 & 1.10 & 0.31 & \\
\hline & & Batch $100 \%$ & 0.73 & 0.92 & 0.39 & 0.58 & 1.07 & 0.75 & 0.56 & 0.58 & 1.10 & 0.82 & 0.83 & 0.85 & 0.31 & \\
\hline \multirow{6}{*}{$\stackrel{\sim}{\leftarrow}$} & \multirow{3}{*}{ Unoptimal } & No Batch & 0.67 & 0.85 & 0.00 & 0.28 & 0.56 & 061 & 0.80 & 0.60 & 0.73 & 0.75 & 0.77 & 0.78 & 0.31 & \\
\hline & & Butch $50 \%$ & 0.61 & 0.93 & 0.00 & 0.28 & 0.59 & 0.59 & 0.60 & 0.60 & 0.82 & 0.83 & 0.91 & 0.91 & 0.32 & \\
\hline & & Batch $100 \%$ & 0.61 & 0.83 & 0.00 & 0.21 & 0.62 & 0.51 & 0.56 & 0.55 & 1.04 & 0.98 & 0.82 & 0.92 & 0.25 & \\
\hline & \multirow{3}{*}{ Optimal } & No Batch & 056 & 0.62 & 0.07 & 0.28 & 0.52 & 0.45 & 0.44 & 0.44 & 0.42 & 0.44 & 0.79 & 0.79 & 0.23 & \\
\hline & & Batch $50 \%$ & 0.61 & 0.81 & 0.10 & 0.26 & 0.61 & 0.44 & 0.50 & 0.47 & 0.83 & 0.69 & 0.78 & 0.76 & 0.24 & \\
\hline & & Batch $100 \%$ & 0.61 & 0.93 & 0.11 & 0.27 & 0.87 & 0.49 & 0.54 & 0.51 & 1.14 & 1.16 & 0.94 & 0.94 & 0.28 & \\
\hline
\end{tabular}

\section{Demand Process: MA(1) $\theta:-\mathbf{0 . 2}$}

\begin{tabular}{|c|c|c|c|c|c|c|c|c|c|c|c|c|c|c|c|c|}
\hline $\begin{array}{l}\text { Lead } \\
\text { Time }\end{array}$ & Ordering & Butch & $\begin{array}{l}\text { Raw } \\
\text { Time }\end{array}$ & FT Total & FT 기 & FT 28: & $\begin{array}{l}\text { FT 80\% } \\
\text { Energy }\end{array}$ & AR ? & AR 28: & AR AIC & ACF $T$ & ACF 28 & CCF 7 & CCF 28 & $\begin{array}{l}\text { DWT Haar } \\
\text { (Level 5) }\end{array}$ & $\begin{array}{l}\text { DWT Haar } \\
\text { (Love) } \\
\text { Shennon) }\end{array}$ \\
\hline \multirow{6}{*}{$\begin{array}{l}\text { ले } \\
\text { 占 }\end{array}$} & \multirow{3}{*}{ Unoptimal } & No Batch & 0.91 & 1.47 & 0.17 & 080 & 152 & 1.12 & 1.03 & 1.05 & 1.64 & 0.91 & 138 & 1.58 & 0.28 & 0.68 \\
\hline & & Batch 50\% & 0.88 & 1.41 & 0.18 & 089 & 1.54 & 0.68 & 0.62 & 0.63 & 1.82 & 1.05 & 1.35 & 165 & 032 & 0.68 \\
\hline & & Batch $100 \%$ & 0.88 & 1.20 & 0.28 & 086 & 1.36 & 0.64 & 0.56 & 0.57 & 1.31 & 0.77 & 1.25 & 4.45 & 0.34 & \\
\hline & \multirow{3}{*}{ Optimal } & No Batch & 0.85 & 203 & 030 & 968 & 201 & 0.03 & ठब्या & 0.85 & 3.77 & 1.40 & 1.73 & 290 & 0.50 & $\overline{0.63}$ \\
\hline & & Batch 50\% & 090 & 1.35 & 0.43 & 0.82 & 1.46 & 0.48 & 0.40 & 0.40 & 1.25 & 0.78 & 9.57 & 208 & 0.35 & \\
\hline & & Batch $100 \%$ & 0.90 & 1.07 & 0.36 & 057 & 1.11 & 029 & 0.28 & 028 & 0.43 & 030 & 1.21 & 135 & 0.35 & \\
\hline \multirow{6}{*}{$\begin{array}{l}\stackrel{\mathscr{N}}{\mathbf{N}} \\
\mathbf{5}\end{array}$} & \multirow{3}{*}{ Unoptimal } & No Batch & 0.3 & 1.48 & 0.11 & 084 & 1.61 & 0.82 & 0.78 & 0.77 & 1.57 & 1.18 & 1.39 & 1.88 & 0.30 & \\
\hline & & Batch 50\% & 0.82 & 1.44 & 0.14 & 0.55 & 1.58 & 0.55 & 0.53 & 0.53 & 1.70 & 1.24 & 141 & 1.71 & 0.31 & \\
\hline & & Butch $100 \%$ & 0.83 & 1.27 & 0.25 & 0.55 & 1.35 & 0.46 & 0.43 & 0.42 & 110 & 0.83 & 1.22 & 1.36 & 032 & \\
\hline & \multirow{3}{*}{ Optimal } & No Batch & 070 & 183 & 0.18 & 9.23 & 1.80 & 0.73 & 0.55 & 0.80 & 2.16 & 1.27 & 1.90 & 2.51 & 0.39 & \\
\hline & & Batch $50 \%$ & 0.83 & 1.30 & 0.21 & 0.89 & 1.39 & 0.42 & 0.38 & 0.36 & 1.02 & 0.77 & 1.02 & 1.87 & 029 & \\
\hline & & Batch $100 \%$ & 084 & 1.10 & 0.23 & 0.49 & 1.13] & 0.23 & 0.25 & 0.25 & 0.35 & 0.35 & 129 & 1.36 & 0.30 & \\
\hline \multirow{6}{*}{$\underset{\leftarrow}{\sim}$} & \multirow{3}{*}{ Unoptimal } & No Balkh & 084 & 1.33 & 0.18 & 0.15 & 1.35 & 0.68 & 0.03 & 0.64 & 1.24 & 1.17 & 1.41 & 1.45 & 0.24 & \\
\hline & & Batch $50 \%$ & 0.71 & 1.30 & 0.30 & 0.31 & 1.35 & 0.74 & 0.70 & 0.72 & 1.04 & 1.00 & 1.58 & 181 & 0.34 & \\
\hline & & Batch $100 \%$ & 0.71 & 1.25 & 0.37 & 0.45 & 1.29 & 0.61 & 0.81 & 0.61 & 0.85 & 0.80 & 1.25 & 1.28 & 0.38 & \\
\hline & \multirow{3}{*}{ Optimal } & No Batch & 0.52 & 1.18 & 0.04 & 0.28 & 1.14 & 0.53 & 0.52 & 0.52 & 0.91 & 0.88 & 1.11 & 1.12 & 0.18 & \\
\hline & & Batch $50 \%$ & 0.65 & 9.11 & 0.23 & 0.23 & 1.11 & 0.49 & 0.51 & 0.50 & 0.60 & 0.61 & 1.33 & 1.34 & 0.24 & \\
\hline & & Batch $100 \%$ & 0.73 & 1.16 & 0.31 & 0.34 & 1.13 & 021 & 0.35 & 0.26 & 0.28 & 0.41 & 1.23 & 1.24 & 0.27 & \\
\hline
\end{tabular}

Demand Process: MA(2) $\theta_{1}: 0.1 \theta_{2}:-0.8$

\begin{tabular}{|c|c|c|c|c|c|c|c|c|c|c|c|c|c|c|c|c|}
\hline $\begin{array}{l}\text { Lead } \\
\text { Time }\end{array}$ & Ordering & Batch & $\begin{array}{l}\text { Raw } \\
\text { Time }\end{array}$ & Total & FT T & FT 28: & $\begin{array}{l}\text { FT 80\%/ } \\
\text { Enargy }\end{array}$ & $A R=1$ & AR 28 & AR AIC & ACF 7 & ACF 28 & CCF 7 & CCF 28 & $\begin{array}{r}\text { OWT Hand } \\
\text { (Lovel 5) }\end{array}$ & $\begin{array}{l}\text { DWT Heat } \\
\text { (Leve) } \\
\text { Shennon) }\end{array}$ \\
\hline \multirow{4}{*}{$\begin{array}{l}\stackrel{ \pm}{7} \\
\stackrel{5}{\lrcorner}\end{array}$} & & No Borch & 0.83 & 1.63 & 020 & 0.82 & 1.79 & 1.18 & 120 & 1.20 & 200 & 0.98 & 138 & $\begin{array}{l}153 \\
157\end{array}$ & 0.29 & 0.87 \\
\hline & Unoptimal & $\begin{array}{l}\text { Batch } 50 \% \\
\text { Butch 100\% }\end{array}$ & $\begin{array}{l}0.80 \\
0.80\end{array}$ & $\begin{array}{l}1.50 \\
1.44\end{array}$ & $\begin{array}{l}0.15 \\
0.21\end{array}$ & $\begin{array}{l}0.88 \\
0.88\end{array}$ & $\begin{array}{l}1.70 \\
1.63\end{array}$ & $\begin{array}{l}1.20 \\
1.23\end{array}$ & $\begin{array}{l}1.36 \\
1.27\end{array}$ & $\begin{array}{l}1.37 \\
1.28\end{array}$ & $\begin{array}{l}1.99 \\
1.72\end{array}$ & $\begin{array}{l}1.09 \\
0.97\end{array}$ & $\begin{array}{r}1.30 \\
1.24\end{array}$ & $\begin{array}{l}1.57 \\
1.44\end{array}$ & $\begin{array}{l}0.29 \\
0.29\end{array}$ & $\begin{array}{l}0.64 \\
0.86\end{array}$ \\
\hline & & No Batch & 0.05 & 1.95 & 0.28 & 130 & 2.10 & 0.98 & 0.87 & 0.97 & 366 & 1.31 & 154 & 2.37 & 0.48 & 0.58 \\
\hline & Optimal & Bat & $\begin{array}{l}0.87 \\
0.80\end{array}$ & $\begin{array}{l}1.61 \\
1.25\end{array}$ & $\begin{array}{l}0.39 \\
0.34\end{array}$ & $\begin{array}{l}1.10 \\
0.82\end{array}$ & $\begin{array}{r}1.83 \\
1.35\end{array}$ & $\begin{array}{l}1.02 \\
0.82\end{array}$ & $\begin{array}{l}1.15 \\
0.96\end{array}$ & $\begin{array}{l}1.15 \\
0.97\end{array}$ & $\begin{array}{l}2.08 \\
0.86\end{array}$ & $\begin{array}{l}1.09 \\
0.59\end{array}$ & $\begin{array}{l}1.59 \\
1.27\end{array}$ & $\begin{array}{l}228 \\
151\end{array}$ & $\begin{array}{l}0.36 \\
0.32\end{array}$ & $\begin{array}{l}0.58 \\
0.50\end{array}$ \\
\hline \multirow{5}{*}{$\begin{array}{l}\underset{\mathcal{N}}{5} \\
\stackrel{\sim}{5}\end{array}$} & & No Bntc & 0.84 & 1.50 & 0.09 & 0.58 & 1.79 & 0.80 & 0.92 & 0.82 & 170 & 1.10 & 1.28 & 1.47 & 0.28 & 0.58 \\
\hline & Unoptimal & & $\begin{array}{l}0.82 \\
0.82\end{array}$ & $\begin{array}{c}1.51 \\
1.0\end{array}$ & $\begin{array}{l}0.12 \\
0.22\end{array}$ & $\begin{array}{l}0.51 \\
0.49\end{array}$ & $\begin{array}{r}1.80 \\
150\end{array}$ & $\begin{array}{l}0.79 \\
0.88\end{array}$ & 0.96 & 0.98 & 181 & 129 & 1.37. & 1.64 & 0.29 & $\begin{array}{l}0.57 \\
0.62\end{array}$ \\
\hline & & $\frac{\text { Bat }}{\text { No }}$ & 0.75 & $\frac{1.40}{1.80}$ & 0.18 & 121 & 2.05 & 0.74 & 0.74 & 0.73 & 2.62 & 1.43 & 180 & 231 & 0.38 & 049 \\
\hline & Optimal & & 0.80 & 1.54 & 0.18 & 0.84 & 4.76 & 0.82 & 0.86 & & 1.70 & 1.10 & 1.72 & & & 045 \\
\hline & & Bat & 0.85 & 120 & 0.22 & 0.52 & 1.00 & 0.75 & & & 0.83 & 0.88 & 1.43 & 1.55 & & 0.45 \\
\hline & & & & & 0.00 & 0.24 & & 0.71 & 0.73 & & 1.77 & 1.60 & & 1.29 & & 037 \\
\hline & Unoptimal & & 0.70 & 1.57 & 0.20 & 0. & 1.83 & 0.98 & 1.02 & & 8 & 1.45 & 1.50 & 1.52 & & 0.43 \\
\hline & & Baten $100 \%$ & 072 & 1.47] & 034 & 038 & 1,73 & 1.17 & 1.18 & 1.19 & 1.45 & 2 & 1.27 & 1.28 & 0.31 & 0.46 \\
\hline & & No Batc & 054 & 1.54 & 0.11 & 0.4 & 1.65 & 0.64 & 0.66 & 0.60 & 1.48 & 1.40 & 1.15 & 1.16 & & 0.33 \\
\hline & Optimal & & & 1.40 & 0.08 & 0.36 & 1.54 & 100 & 1.24 & 1.2 & 12 & 1.15 & 1.2 & $1:$ & & 0.35 \\
\hline & & laten $100 \%$ & 0.74 & 1.38 & 0.27 & 028 & 144 & 1.18 & 1.41 & 1.41 & 102 & 0.98 & 130 & 131 & 0.24 [ & 0.42 \\
\hline
\end{tabular}

Raw Time: Based on data in the time domain

FT Tolal, FT 7, FT 28 : Based on amplitudes of total, first 7 and first 28 frequencies atter fourier transform

FT B0\% Energy - Based on amplitudes of first as many frequencies as have $80 \%$ of the energy in the data after founer transform

AR 7 AR 28 : Based on autoregressive parameters (AR) of time series represented as AR models of order 7 and 28

AR AIC Based on autoregressive parameters (AR) of time series represented as AR models of order defined by minimising Akaike information criteria (AIC) ACF 7. ACF 28. Based on autocomelations upto 1307 and 1 ing 28

CCF 7 CCF 28 : Based on dyadic cross correlations between variables upto max \pm 7 lags and \pm 28 lags

DWT (Level 5): Based on coefficients from discrete wavelet decomposition upto level 5 using Haar wavelet

DWT (Level Shannon): Based on coefficients from discrete wavelet decomposition upto level defined by minimum Shannon entropy using Haar wavelet 


\section{Appendix I continued}

\section{I.2 Coefficient of variation of index values}

\section{Demand Process: AR(2) $\rho_{1}: 0.1 \rho_{2}:-0.8$}

\begin{tabular}{|c|c|c|c|c|c|c|c|c|c|c|c|c|c|c|c|c|}
\hline $\begin{array}{l}\text { Lead } \\
\text { Timo }\end{array}$ & Ordering & Batch & $\begin{array}{l}\text { Ran } \\
\text { TIme }\end{array}$ & FT Total & FT 7 & FT 28: & $\begin{array}{l}\text { FT 80\% } \\
\text { Energy }\end{array}$ & $A R 7$ & AR 28 & AR AIC & ACF 7 & ACF 28 & CCF 7 & CCF 28 & $\begin{array}{r}\text { OWT Haar } \\
\text { (Lovel 5) }\end{array}$ & $\begin{array}{l}\text { DWT HaAn } \\
\text { Revel } \\
\text { Shannon }\end{array}$ \\
\hline \multirow{6}{*}{$\stackrel{5}{5}$} & \multirow{3}{*}{ Unoptimal } & No Batch & 0.03 & 0.08 & 0.38 & 009 & 0.20 & 0.14 & 0.19 & 0.14 & 0.16 & 0.13 & 0.06 & 0.06 & 0.15 & 0.15 \\
\hline & & Batch 50\% & 0.03 & 0.09 & 0.37 & 0.12 & 0.18 & 0.11 & 0.15 & 0.15 & 0.12 & 0.14 & 0.07 & 0.08 & 0.14 & 0.10 \\
\hline & & Batch $100 \%$ & 0.03 & 0.09 & 0.25 & 0.12 & 0.16 & 0.12 & 0.13 & 014 & 0.15 & 0.14 & 0.09 & 0.10 & 0.24 & 0.12 \\
\hline & \multirow{3}{*}{ Optimal } & No Barch & 0.03 & 0.13 & 0.30 & 0.16 & 0.20 & 0.22 & 0.21 & 0.21 & 0.23 & 0.20 & 0.00 & 0.11 & 0.11 & 0.15 \\
\hline & & Batch $50 \%$ & 0.03 & 0.09 & 0.22 & 0.14 & 0.15 & 0.08 & 0.10 & 0.10 & 0.09 & 0.12 & 0.08 & 0.12 & 0.15 & 0.08 \\
\hline & & Batch $100 \%$ & 0.03 & 0.08 & 0.28 & 0.24 & 0.16 & 0.11 & 0.22 & 0.22 & 0.10 & 0.14 & 0.11 & 0.12 & 0.18 & 0.09 \\
\hline \multirow{6}{*}{$\underset{\mathfrak{N}}{\stackrel{\mathfrak{V}}{5}}$} & \multirow{3}{*}{ Unoptimal } & No Baich & 0.03 & 0.09 & 0.37 & 0.12 & 0.18 & 0.13 & 0.14 & 0.14 & 0.14 & 0.14 & 0.07 & 0.07 & 0.16 & 0.08 \\
\hline & & Baten $50 \%$ & 0.03 & 0.08 & 0.43 & 0.12 & 0.16 & 0.06 & 0.10 & 0.11 & 0.13 & 0.14 & 0.08 & 0.08 & 0.15 & 0.10 \\
\hline & & Batch $100 \%$ & 0.03 & 0.10 & 0.29 & 0.17 & 0.18 & 009 & 0.11 & 0.11 & 0.16 & 0.17 & 0.10 & 0.10 & 0.17 & 0.07 \\
\hline & \multirow{3}{*}{ Optimal } & No Baाch & 0.03 & 0.00 & 0.31 & 0.14 & 0.15 & 0.18 & 0.18 & 0.20 & 0.15 & 0.12 & 0.08 & 0.09 & 0.12 & 0.14 \\
\hline & & Batch $50 \%$ & 0.03 & 0.00 & 0.16 & 0.14 & 0.18 & 0.05 & 0.09 & 0.09 & 0.09 & 0.10 & 0.09 & 0.10 & 0.15 & 0.07 \\
\hline & & Batch $100 \%$ & 0.03 & 0.10 & 0.28 & 0.15 & 0.16 & 000 & 0.18 & 0.15 & 0.12 & 0.16 & 0.11 & 0.11 & 0.15 & 0.12 \\
\hline \multirow{6}{*}{$\stackrel{\simeq}{5}$} & \multirow{3}{*}{ Unoptimal } & No Balch & 0.02 & 0.00 & 0.56 & 0.13 & 0.14 & 0.15 & 0.15 & 0.15 & 0.11 & 0.10 & 0.07 & 0.07 & 0.18 & 0.21 \\
\hline & & Batch $50 \%$ & 0.02 & 0.07 & 0.41 & 0.16 & 0.14 & 0.00 & 0.09 & 0.09 & 0.11 & 0.11 & 0.06 & 0.06 & 0.21 & 0.12 \\
\hline & & Batch $100 \%$ & 0.02 & 0.08 & 0.34 & 0.10 & 0.14 & 0.10 & 0.10 & 0.10 & 0.15 & 0.15 & 0.05 & 0.05 & 0.18 & 0.07 \\
\hline & \multirow{3}{*}{ Optimal } & No Batch & 0.00 & 0.05 & 0.44 & 0.00 & 0.11 & 0.20 & 0.21 & 0.21 & 0.10 & 0.10 & 0.07 & 0.07 & 0.16 & 0.29 \\
\hline & & Batch 50\% & 0.02 & 0.07 & 0.38 & 0.14 & 0.13 & 0.09 & 0.09 & 0.09 & 0.13 & 0.11 & 0.08 & 0.08 & 0.15 & 0.22 \\
\hline & & Batch $100 \%$ & 0.02 & 0.07 & 0.49 & 0.21 & 0.14 & 0.16 & 0.13 & 0.14 & 0.12 & 0.10 & 0.07 & 0.07 & 0.19 & 0 \\
\hline
\end{tabular}

\section{Demand Process: MA(1) $\theta:-0.2$}

\begin{tabular}{|c|c|c|c|c|c|c|c|c|c|c|c|c|c|c|c|c|}
\hline $\begin{array}{l}\text { Lead } \\
\text { Time }\end{array}$ & Ordering & Batch & $\begin{array}{l}\text { Rane } \\
\text { Time }\end{array}$ & FT Total & FT 7 & FT 28 & $\begin{array}{l}\text { FT 80\% } \\
\text { Energy }\end{array}$ & AR 7 & AR 28 & AR AIC & ACF 7 & ACF 28 & CCF 7 & CCF 28 & $\begin{array}{r}\text { DWT Haar } \\
\text { (Level S) }\end{array}$ & $\begin{array}{l}\text { DWT Haar } \\
\text { (Leve? } \\
\text { Shanneng) }\end{array}$ \\
\hline \multirow{6}{*}{$\begin{array}{l}\text { लू } \\
\stackrel{⺊}{-}\end{array}$} & \multirow{3}{*}{ Unoptimal } & No Batch & 0.02 & 0.06 & 0.39 & 0.17 & 0.07 & 0.20 & 0.18 & 0.19 & 0.15 & 0.16 & 0.07 & 0.07 & 0.20 & 0.26 \\
\hline & & Batch $50 \%$ & 0.01 & 0.06 & 0.41 & 0.16 & 0.10 & 0.12 & 0.14 & 0.14 & 0.16 & 0.18 & 0.06 & 0.05 & 0.17 & 0.16 \\
\hline & & Batch $100 \%$ & 0.02 & 0.07 & 0.33 & 0.15 & 0.09 & 0.23 & 0.28 & 0.25 & 0.20 & 0.18 & 0.06 & 0.07 & 0.20 & 0.17 \\
\hline & \multirow{3}{*}{ Optimal } & No Bnich & 001 & 0.08 & 0.30 & 0.10 & 0.07 & 0.30 & 0.28 & 0.28 & 0.13 & 0.15 & 0.07 & 0.09 & 0.14 & 0.19 \\
\hline & & Batch $50 \%$ & 0.02 & 0.08 & 0.25 & 0.17 & 0.10 & 0.14 & 0.15 & 0.14 & 0.21 & 0.20 & 0.07 & 0.09 & 0.11 & 0.21 \\
\hline & & Batch $100 \%$ & 002 & 0.07 & 0.29 & 0.21 & 0.11 & 0.29 & 0.21 & 0.22 & 0.34 & 0.27 & 0.08 & 0.11 & 0.12 & 0,08 \\
\hline \multirow{6}{*}{$\begin{array}{l}\underset{N}{N} \\
\stackrel{5}{J}\end{array}$} & \multirow{3}{*}{ Unoptimal } & No Batch & 0.02 & 0.00 & 0.56 & 0.17 & 0.08 & 0.17 & 0.19 & 0.20 & 0.15 & 0.15 & 0.08 & 0.09 & 0.18 & 0.23 \\
\hline & & Batch $50 \%$ & 0.09 & 0.05 & 0.32 & 0.20 & 0.07 & 0.11 & 0.13 & 0.13 & 0.12 & 0.13 & 0.06 & 0.06 & 0.21 & 0.19 \\
\hline & & Batch $100 \%$ & 0.01 & 0.05 & 0.20 & 0.16 & 000 & 020 & $0.21]$ & 0.21 & 0.15 & 0.15 & 0.05 & 0.05 & 0.14 & 0.19 \\
\hline & \multirow{3}{*}{ Optimal } & No Batch & 0.01 & 0.05 & 0.30 & 0.11 & 0.07 & 0.28 & 026 & 026 & 0.12 & 0.11 & 0.07 & 0.07 & 0.15 & 0.17 \\
\hline & & Batch 50\% & 0.02 & 0.05 & 0.37 & 0.15 & 0.07 & 000 & 0.14 & 0.14 & 0.12 & 0.12 & 0.06 & 0.06 & 0.10 & 0 \\
\hline & & Basch $100 \%$ & 001 & 0.04 & 0.35 & 0.16 & 0.08 & 0.25 & 0.17 & 0.17 & 0.31 & 026 & 0.07 & 0.08 & 0.17 & 0.13 \\
\hline \multirow{6}{*}{$\underset{5}{\stackrel{5}{5}}$} & \multirow{3}{*}{ Unoptimal } & No Balch & 002 & 0.05 & 026 & 0.14 & 0.06 & 0.14 & 0.16 & 0.15 & 0.10 & 0.10 & 0.08 & 0.09 & 0.17 & 0.21 \\
\hline & & Batch 50\% & 0.01 & 0.00 & 018 & 0.15 & 0.07 & 0.09 & 0.08 & 0.08 & 0.12 & 0.11 & 0.06 & 0.06 & 0.14 & 0.21 \\
\hline & & Batch $100 \%$ & 001 & 0.04 & 0.22 & 0.13 & 0.06 & 0.11 & 0.09 & 0.09 & 0.10 & 0.09 & 0.04 & 0.04 & 0.17 & 0.09 \\
\hline & \multirow{3}{*}{ Optimal } & No Batch & 0.02 & 0.05 & 0.46 & 0.17 & 0.06 & 0.21 & 0.21 & 021 & 0.09 & 0.08 & 0.07 & 0.07 & 0.21 & \\
\hline & & Batch 50\% & 0.03 & 0.04 & 020 & 0.13 & 0.06 & 0.14 & 0.14 & 0.13 & 009 & 008 & 0.07 & 0.07 & 0.15 & \\
\hline & & Batch $100 \%$ & 0.01 & 0.05 & 0.26 & 0.15 & 0.07 & 0.31 & 0.18 & 0.28 & 030 & 0.15 & 0.04 & 0.04 & 0.20 & \\
\hline
\end{tabular}

\section{Demand Process: MA(2) $\theta_{1}: 0.1 \theta_{2}:-0.8$}

\begin{tabular}{|c|c|c|c|c|c|c|c|c|c|c|c|c|c|c|c|c|}
\hline $\begin{array}{l}\text { Loed } \\
\text { Timm }\end{array}$ & Ordering & Batch & $\begin{array}{l}\text { Raw } \\
\text { Time }\end{array}$ & FT Total & FT 7 & FT 28 & $\begin{array}{l}\text { FT 80\% } \\
\text { Energy }\end{array}$ & AR 7 & AR 28 & AR AIC & ACF 7 & ACF 28 & CCF 7 & CCF 28 & $\begin{array}{r}\text { DWT Haar } \\
\text { (Level S) }\end{array}$ & $\begin{array}{l}\text { DWT Haary } \\
\text { (Level } \\
\text { shinnon) }\end{array}$ \\
\hline \multirow{6}{*}{ 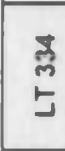 } & \multirow{3}{*}{ Unoptimal } & No Banch & 0.03 & 0.04 & 0.37 & 0.13 & 0.07 & 0.29 & 0.28 & 0.28 & 0.12 & 0.11 & 0.06 & 0.05 & 0.26 & 0.18 \\
\hline & & Batch $50 \%$ & 0.01 & 0.04 & 0.36 & 0.13 & 0.07 & 0.12 & 0.12 & 0.12 & 0.13 & 0.15 & 0.05 & 0.05 & 0.16 & 0.20 \\
\hline & & Batch $100 \%$ & 0.02 & 0.04 & 0.31 & 0.09 & 0.07 & 0.10 & 0.11 & 0.11 & 0.11 & 0.11 & 0.05 & 0.05 & 0.15 & 0.18 \\
\hline & \multirow{3}{*}{ Optimal } & No Batch & 0.01 & 0.06 & 0.28 & 0.14 & 0.08 & 0.28 & 0.24 & 0.24 & 0.10 & 0.13 & 0.07 & 0.11 & 0.14 & 0.11 \\
\hline & & Batch 50\% & 0.02 & 0.06 & 0.30 & 0.13 & 0.08 & 0.09 & 0.12 & 0.12 & 0.12 & 0.15 & 0.07 & 0.08 & 0.14 & 0.11 \\
\hline & & Batch $100 \%$ & 0.01 & 0.07 & 0.29 & 0.18 & 0.08 & 0.12 & 0.12 & 0.12 & 0.21 & 0.18 & 0.08 & 0.08 & 0.13 & 0.07 \\
\hline \multirow{6}{*}{$\stackrel{\text { ป }}{5}$} & \multirow{3}{*}{ Unoptimal } & No Batch & 0.02 & 0.05 & 0.44 & 0.18 & 0.08 & 0.11 & 0.11 & 0.11 & 0.12 & 0.15 & 0.08 & 0.09 & 0.21 & 0.22 \\
\hline & & Batch $50 \%$ & 0.01 & 0.05 & 0.33 & 0.21 & 0.08 & 0.09 & 0.11 & 0.11 & 0.13 & 0.13 & 0.08 & 0.09 & 0.18 & 0.17 \\
\hline & & Batch $100 \%$ & 0.01 & 004 & 0.32 & 0.17 & 0.08 & 0.13 & 0.15 & 0.15 & 0.10 & 0.09 & 0.06 & 0.05 & 0.18 & 0.17 \\
\hline & \multirow{3}{*}{ Optimal } & No Batch & 0.01 & 0.05 & 0.32 & 0.10 & 0.08 & 0.20 & 0.20 & 0.20 & 0.11 & 0.12 & 0.07 & 0.09 & 0.15 & 0.15 \\
\hline & & Batch 50\% & 0.02 & 0.05 & 0.34 & 0.14 & 0.07 & 0.12 & 0.12 & 0.12 & 0.11 & 0.11 & 0.05 & 0.08 & 0.16 & 0.15 \\
\hline & & Batch $100 \%$ & 0.01 & 0.08 & 0.25 & 0.21 & 0.10 & 0.12 & 0.13 & 0.12 & 0.18 & 0.13 & 0.07 & 0.07 & 0.15 & 0.14 \\
\hline \multirow{6}{*}{$\stackrel{2}{5}$} & \multirow{3}{*}{ Unoptimal } & No Batch & 0.02 & 0.05 & 0.58 & 0.20 & 0.07 & 0.11 & 0.10 & 0.11 & 0.11 & 0.11 & 0.09 & 0.09 & 0.18 & 0.17 \\
\hline & & Batch 50\% & 0.03 & 0.04 & 0.32 & 0.14 & 0.08 & 0.05 & 0.06 & 006 & 0.11 & 0.11 & 0.07 & 0.07 & 0.19 & 0.20 \\
\hline & & Batch $100 \%$ & 0.01 & 0.03 & 0.21 & 014 & 0.07 & 0.05 & 0.05 & 005 & 0.00 & 0.00 & 0.04 & 0.04 & 0.12 & 0.09 \\
\hline & \multirow{3}{*}{ Optimal } & No Batch & 0.02 & 0.05 & 0.29 & 0.16 & 0.08 & 0.14 & 0.13 & 0.14 & 0.10 & 0.10 & 0.07 & 0.07 & 0.16 & 0.12 \\
\hline & & Balch $50 \%$ & 0.02 & 0.04 & 0.49 & 0.18 & 0.06 & 0.06 & 0.06 & 0.08 & 0.10 & 0.09 & 0.05 & 0.05 & 0.16 & 0.16 \\
\hline & & Batch $100 \%$ & 001 & 005 & 024 & 0.13 & 0.08 & 0.08 & 0.05 & 0.05 & 0.10 & 0.10 & 0.05 & 0.05 & 0.16 & 005 \\
\hline
\end{tabular}




\section{Appendix J}

Index values for the Beer game system based on different feature parameter combinations: Only order and inventory variables used in the index computation

\section{J.1 Average index values}

\section{Demand Process: AR(2) $\rho_{1}: 0.1 \rho_{2}:-0.8$}

\begin{tabular}{|c|c|c|c|c|c|c|c|c|c|c|c|c|c|c|c|c|}
\hline $\begin{array}{l}\text { Loed } \\
\text { rime }\end{array}$ & Ordering & Batch & $\begin{array}{l}\text { Raw } \\
\text { Time }\end{array}$ & FT Total & FT 7 & FT 28: & $\begin{array}{l}\text { FT sory } \\
\text { Energy }\end{array}$ & AR ? & AR 28 & AR AIC & ACF 7 & ACF 28 & CCF 7 & CCF 28 & $\begin{array}{l}\text { DWT Haar } \\
\text { (Level 5) }\end{array}$ & $\begin{array}{l}\text { DWT Haar } \\
\text { (Level) } \\
\text { Shnanon) }\end{array}$ \\
\hline \multirow{6}{*}{$\begin{array}{l}\text { ले } \\
\stackrel{5}{-}\end{array}$} & \multirow{3}{*}{ Unoptimal } & No Balch & 070 & 1.45 & 0.42 & 1.56 & 1.40 & 1.49 & 1.32 & 1.34 & 258 & 1.55 & 1.18 & 1.52 & 0.49 & 0.50 \\
\hline & & Betch 50\% & 0.84 & 1.43 & 0.34 & 1.07 & 2.25 & 0.91 & 086 & 0.86 & 2.05 & 1.27 & 1.30 & 1.50 & 0.38 & 0.45 \\
\hline & & Betch $100 \%$ & 1.04 & 1.41 & 0.46 & 0.80 & 3.43 & 0.82 & 0.78 & 0.78 & 1.77 & 0.80 & 1.41 & 1.00 & 0.30 & 0.57 \\
\hline & \multirow{3}{*}{ Optimal } & No Batch & 0.61 & 1.30 & 0.85 & 1.80 & 074 & 0.74 & 0.78 & 0.78 & 1.40 & 1.00 & 1.33 & 2.11 & 0.44 & 0.43 \\
\hline & & Batch 50\% & 0.83 & 1.19 & 0.43 & 0.71 & 164 & 0.93 & 0.80 & 080 & 1.15 & 0.91 & 1.07 & 1.18 & 0.27 & 0.38 \\
\hline & & Batch $100 \%$ & 0.93 & 1.20 & 0.38 & 0.58 & 2.81 & 0.94 & 0.67 & 0.87 & 100 & 0.67 & 1.33 & 1.40 & 0.28 & 0.38 \\
\hline \multirow{6}{*}{$\underset{\mathcal{N}}{\mathcal{N}}$} & \multirow{3}{*}{ Unoptimal } & No Barch & 0.67 & 1.64 & 0.27 & 1.78 & 1.52 & 1.37 & 1.21 & 1.24 & 3.00 & 1.78 & 1.65 & 2.77 & 0.48 & 0.51 \\
\hline & & Batch 50\% & 0.78 & 1.55 & 0.24 & 0.95 & 2.33 & 0.79 & 0.77 & 0.77 & 250 & 4.73 & 1.65 & 1.81 & 0.35 & 0.41 \\
\hline & & Baten $100 \%$ & 0.91 & 1.31 & 0.30 & 0.60 & 205 & 0.75 & 0.68 & 0.88 & 141 & 0.80 & 1.23 & 1.31 & 0.28 & 0.38 \\
\hline & \multirow{3}{*}{ Optimal } & No Bench & 0.50 & 1.18 & 0.47 & 1.74 & 0.71 & 0.87 & 0.00 & 0.88 & 1.38 & 1.02 & 1.53 & 9.84 & 0.43 & 0.40 \\
\hline & & Bateh $50 \%$ & 0.73 & 1.23 & 0.41 & 0.60 & 1.64 & 0.80 & 0.78 & 0.78 & 1.44 & 1.44 & 1.37 & 1.45 & 0.27 & 0.38 \\
\hline & & Barch $100 \%$ & $0 . \infty$ & 1.19 & 0.30 & 0.49 & 228 & 0.85 & 0.74 & 0.75 & 1.05 & 0.77 & 1.17 & 1.19 & 0.24 & 0.41 \\
\hline \multirow{6}{*}{$\stackrel{2}{5}$} & \multirow{3}{*}{ Unoptimal } & No Batch & 0.87 & 0.80 & 0.21 & 0.67 & 0.67 & 1.05 & 0.05 & 0.99 & 0.7 & 0.79 & 0.89 & 0.89 & 0.37 & 0.48 \\
\hline & & Batch 50\% & 0.71 & 1.08 & 0.17 & 0.42 & 0.91 & 0.53 & 0.54 & 0.54 & 1.07 & 1.10 & 1.04 & 1.04 & 0.34 & 0.43 \\
\hline & & Batch $100 \%$ & 078 & 1.10 & 0.08 & 0.19 & 1.32 & 0.46 & 0.50 & 0.48 & 1.37 & 1.37 & 1.16 & 1.16 & 0.21 & 0.37 \\
\hline & \multirow{3}{*}{ Optimal } & No Batch & 0.48 & 0.68 & 0.27 & 0.80 & 0.48 & 0.42 & 0.41 & 0.41 & 0.4 & 0.47 & 0.90 & 0.90 & 0.28 & 0.29 \\
\hline & & Batch $50 \%$ & 0.55 & 0.83 & 0.21 & 0.48 & 0.89 & 0.37 & 0.42 & 0.40 & 0.67 & 0.73 & 0.84 & 0.84 & 0.26 & 0.34 \\
\hline & & Batch $100 \%$ & 0.67 & 1.13 & 0.15 & 0.35 & 1.08 & 0.39 & 0.42 & 0.40 & 1.33 & 1.41 & 0.99 & 0.99 & 0.28 & 0.54 \\
\hline
\end{tabular}

Demand Process: MA(1) $\theta:-0.2$

\begin{tabular}{|c|c|c|c|c|c|c|c|c|c|c|c|c|c|c|c|c|}
\hline Load & Ordering & Baten & $\begin{array}{l}\text { Raw } \\
\text { Time }\end{array}$ & & FTT & FT 2E & $\begin{array}{l}\text { FT sox } \\
\text { Energy }\end{array}$ & AR 7 & AR 28 & AR AIC & ACF 7 & ACF 28 & CCF 7 & CCF 28 & $\begin{array}{l}\text { OWT Heart } \\
\text { (Lovel 5) }\end{array}$ & $\begin{array}{l}\text { DWT Haa } \\
\text { (Loval) } \\
\text { Shinnion) }\end{array}$ \\
\hline \multirow{5}{*}{ ల్ల్ } & & No 8sich & 0.82 & 1.93 & 0.33 & 9.42 & 2.17 & 1.72 & 1.48 & 1.56 & 4.20 & 1.94 & 1.74 & 210 & 0.34 & 0.58 \\
\hline & Unoptimal & $\begin{array}{l}\text { Bath } 50 \% \\
\text { Batch } 100 \%\end{array}$ & $\begin{array}{l}0.83 \\
0.90\end{array}$ & $\begin{array}{l}1.62 \\
1.38\end{array}$ & $\begin{array}{l}0.28 \\
0.28\end{array}$ & $\begin{array}{l}1.24 \\
0.93\end{array}$ & $\begin{array}{l}1.87 \\
1.50\end{array}$ & $\begin{array}{l}0.65 \\
0.72\end{array}$ & $\begin{array}{l}0.63 \\
0.88\end{array}$ & $\begin{array}{l}0.63 \\
0.69\end{array}$ & $\begin{array}{l}2.86 \\
1.87\end{array}$ & $\begin{array}{l}1.60 \\
0.85 \\
\end{array}$ & 1.66 & $\begin{array}{l}2.27 \\
1.96\end{array}$ & $\begin{array}{l}0.30 \\
0.29\end{array}$ & $\begin{array}{l}0.57 \\
0.62\end{array}$ \\
\hline & & No Batc & 0.68 & 2.77 & 0.57 & 252 & 270 & 0.90 & $0 \mathrm{OA}$ & 0.96 & 5.86 & 2.30 & 1.95 & 453 & 0.43 & 0.50 \\
\hline & Optimal & Band & 0.76 & 1.40 & 0.58 & 1.03 & 1.58 & 0.50 & 0.52 & 0.50 & 1.44 & 1.111 & 1.63 & 2.28] & 029 & 0.50 \\
\hline & & Baich ? & 0.88 & 0.98 & $031]$ & 0.51 & 1.041 & 0.35 & 0.39 & 0.37 & $0.41]$ & 0.271 & 1.39 & 1.53 & 027 L & 0.44 \\
\hline \multirow{6}{*}{ ก } & & No $B m$ ? & 0.72 & 1.04 & 0.20 & 0.85 & 2.00 & १.177 & 103 & 107 & 3.01 & 1.93 & $1.60 \mid$ & 203 & 0.30 & 0.50 \\
\hline & Unoptimal & & & 1.60 & 0.23 & 0.85 & 1.84 & 0.58 & 0.57 & 0.56 & 2.38 & $1.5 \%$ & & 2.25 & 0.31[ & 0.52 \\
\hline & & Batch $100 x$ & 0.85 & 130 & 0.23 & 0.53 & $1.41 \mid$ & $0.57]$ & 0.57 & 0.57 & 1.23] & 0.90 & 1.65 & 1.88 & 0.29 & 0.58 \\
\hline & & No 80 & 0.61 & 2.47 & 0.38 & 2.14 & 2.44 & 0.78 & 0.72 & 0.73 & 4.59 & 2.14 & 2.75 & 4.03 & 0.37 & 0.47 \\
\hline & Optimal & & 0.89 & 1.41 & 0.36 & 0.00 & 1.60 ] & 0.45 & 0.48 & 0.46 & 1.5 이 & 1.20 & & 2.33 & $0:$ & 0.45 \\
\hline & & Batch $100 \%$ & 0.81 & 1.03 & 0.20 & 0.44 & 1.061 & 0.28 & 0.34 & 0.28 & 0.35 & 0.33 & 1.43 & 1.46 & 0.23 & 0.45 \\
\hline \multirow{5}{*}{ ـ } & & jo & 0.53 & 1.06 & 0.37 & 0.28 & 1.74 & 1.11 & 0.87 & $1 . c$ & 220 & 1.8 & 1.88 & 1.89 & 0.27 & 0.37 \\
\hline & Unoptimal & & 0.61 & 150 & 0 & 0.53 & 1.04 & 0.70 여 & 0.6 & $0 e$ & 1.72 & 1.52 & 2.00 & 26 & & 0.44 \\
\hline & & Batch 1 & 0.72 & 128 & 0.49 & 0.48 & 1.34 & 0.56] & 0.56 & 0.50 & 1.000 & 0.91 & 179 & 1.79 & - 0.35 & 047 \\
\hline & & & 0.41 & 186 & 0.09 & 0.64 & 1.62 & 0.48 & 0.47 & 0.40 & 1.71 & 10 & S & 1 & 0.20 & 0.28 \\
\hline & Optimal & Batch 50\% & 0.53 & 132 & 0.50 & 0.46 & 1.49 & 0.41 & 0.43 & 0.42 & $\begin{array}{l}1.15 \\
0.38\end{array}$ & $\begin{array}{l}112 \\
052\end{array}$ & 14 & 144 & $\begin{array}{l}0.27 \\
0.24\end{array}$ & $\begin{array}{l}0.32 \\
0.411\end{array}$ \\
\hline & & Balch $100 \%$ & & & & & & & & & & & & & & \\
\hline
\end{tabular}

\section{Demand Process: MA(2) $\theta_{1}: 0.1 \theta_{2}:-0.8$}

\begin{tabular}{|c|c|c|c|c|c|c|c|c|c|c|c|c|c|c|c|c|}
\hline $\begin{array}{l}\text { Leed } \\
\text { Time }\end{array}$ & Ordering & Batch & $\begin{array}{l}\text { Rand } \\
\text { Time }\end{array}$ & FT Total & FT 7 & FT 28 & $\begin{array}{l}\text { FT sox } \\
\text { Energy }\end{array}$ & ART & AR 28 & AR AIC & ACF 7 & ACF 28 & CCF 7 & CCF 28 & $\begin{array}{r}\text { DWT Hear } \\
\text { (Level 5) }\end{array}$ & $\begin{array}{l}\text { DWT Haar) } \\
\text { (Level) } \\
\text { Shannon) }\end{array}$ \\
\hline \multirow{6}{*}{$\begin{array}{l}\text { लू } \\
\stackrel{\text { 上 }}{ }\end{array}$} & \multirow{3}{*}{ Unoptimal } & No Batch & 083 & 1.80 & 0.31 & 1.30 & 2.17 & 1.63 & 1.73 & 1.74 & 4.44 & 1.64 & 1.73 & 1.99 & 0.34 & 056 \\
\hline & & Batch $50 \%$ & 0.81 & 1.67 & 0.23 & 1.19 & 2.02 & 1.22 & 1.51 & 1.50 & 3.26 & 1.68 & 1.50 & 2.05 & 0.27 & 0.48 \\
\hline & & Batch $100 \%$ & 0.8 & 1.52 & 0.21 & 0.97 & 1.77 & 1.41 & 165 & 1.67 & 2.25 & 1.19 & 1.61 & 1.92 & 0.26 & 0.58 \\
\hline & \multirow{3}{*}{ Optimal } & No Batch & 0.00 & 2.67 & 0.57 & 222 & 2.83 & 0.93 & 0.95 & 0.24 & 6.94 & 2.51 & 1.81 & 3.69 & 0.49 & 0.46 \\
\hline & & Batch 50\% & 0.72 & 1.81 & 0.70 & 1.42 & 221 & 1.11 & 1.45 & 9.43 & 2.71 & 1.74 & 1.68 & 2.74 & 0.31 & 0.44 \\
\hline & & Batch $100 \%$ & 0.81 & 1.20 & 0.33 & 0.59 & 1.33 & 0.96 & 1.31 & 1.30 & 0.86 & 0.62 & 1.36 & 1.61 & 0.25 & 0.41 \\
\hline \multirow{6}{*}{$\underset{N}{\mathfrak{N}}$} & \multirow{3}{*}{ Unoptimal } & No Belch & 0.72 & 1.75 & 0.13 & 0.82 & 2.00 & 1,10 & 1.24 & 1.24 & 259 & 1.71 & 9.42 & 1.72 & 0.28 & 0.47 \\
\hline & & Batch 50\% & 0.74 & 1.68 & 0.17 & 0.81 & 2.05 & 0.82 & 100 & 1.05 & 260 & 9.71 & 4.63 & 2.05 & 0.29 & 0.46 \\
\hline & & Batch $100 \%$ & 0.80 & 9.43 & 0.24 & 0.49 & 1.65 & 0.97 & 1.20 & 1.17 & 1.55 & 1.12 & 1.50 & 1.82 & 0.27 & 0.50 \\
\hline & \multirow{3}{*}{ Optimal } & No Batch & 050 & 2.59 & 0.36 & 1.6 & 280 & 0.77 & 0.78 & 0.75 & 8.65 & 2.63 & 2.54 & 360 & 0.36 & 0.38 \\
\hline & & Batch 50\% & 0.68 & 1.70 & 0.38 & 1.22 & 2.21 & 0.91 & 128 & 1.23 & 3.20 & 1.88 & 2.12 & 2.82 & 0.27 & 0.38 \\
\hline & & Balch $100 \%$ & 074 & 1.27 & 023 & 0.53 & 1.44 & 0.87 & 1.23 & 1.20 & 0.98 & 0.84 & 1.50 & 1.68 & 0.23 & 0.35 \\
\hline \multirow{6}{*}{$\stackrel{2}{5}$} & \multirow{3}{*}{ Unoptimal } & No Batch & 050 & 1.29 & 0.11 & 0.37 & 2.47 & 0.80 & 0.85 & 0.04 & 3.61 & 2.84 & 9.63 & 1.64 & 0.21 & 0.20 \\
\hline & & Batch 50\% & 0.58 & 1.84 & 0.36 & 0.32 & 2.35 & 1.05 & 1.21 & 1.21 & 3.08 & 2.46 & 1.87 & 1.87 & 0.27 & 0.35 \\
\hline & & Baten $100 \%$ & 0.80 & 1.61 & 048 & 0.43 & 1.91 & 1.28 & 1.47 & 1.40 & 1.97 & 1.65 & $1.7 \mathrm{~d}$ & 1.78 & 0.30 & 0.41 \\
\hline & \multirow{3}{*}{ Optimal } & No Batch & 041 & 2.17 & 0.21 & 0.85 & 2.44 & 0.60 & 0.63 & 0.82 & 3.38 & 300 & 1.80 & 1.80 & 0.23 & 0.25 \\
\hline & & Batch 50\% & 0.51 & 1.71 & 0.16 & 0.55 & 200 & 0.92 & 1.22 & 1.10 & 2.71 & 231 & 1.68 & 1.86 & 0.20 & 0.27 \\
\hline & & Batch $100 \%$ & 0.65 & 1.40 & 0.36 & 0.38 & 160 & 1.10 & 1.42 & 1.40 & 1.80 & 1.43 & 1.55 & 1.55 & 0.22 & 0.31 \\
\hline
\end{tabular}

Raw Time Based on deta in the time domain

7 and firs 28 irequencieas anter fourier transalorm

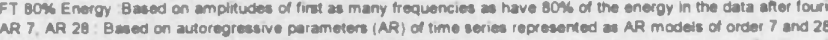

(A)

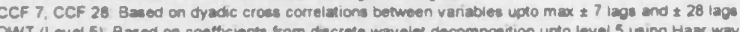

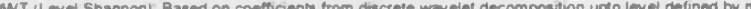




\section{Appendix $\mathbf{J}$ continued}

\section{J.2 Coefficient of variation of index values}

Demand Process: AR(2) $\rho_{1}: 0.1 \rho_{2}:-0.8$

\begin{tabular}{|c|c|c|c|c|c|c|c|c|c|c|c|c|c|c|c|c|}
\hline $\begin{array}{l}\text { Lead } \\
\text { Timo }\end{array}$ & Ordering & Batch & $\begin{array}{l}\text { Raw } \\
\text { Time }\end{array}$ & FT Total & FT t & FT 28 & $\begin{array}{l}\text { FT 80\% } \\
\text { Energy }\end{array}$ & AR T & AR 28 & AR AIC & ACF 7 & ACF 28 & CCF 7 & CCF 28 & $\begin{array}{r}\text { DWT Haar } \\
\text { (Level 5) }\end{array}$ & $\begin{array}{r}\text { DWT Haar } \\
\text { (Level } \\
\text { Shannon! }\end{array}$ \\
\hline \multirow{6}{*}{ స్ } & & No Batch & 0.02 & 0.09 & 0.39 & 0.12 & 0.29 & 0.10 & 0.12 & 0.12 & 0.22 & 0.98 & 0.10 & 0.08 & 0.13 & 0.09 \\
\hline & Unoptimal & Batch 50\% & 0.02 & 0.07 & 0.35 & 0.14 & 0.11 & 0.11 & 0.12 & 0.12 & 0.15 & 0.19 & 0.09 & 0.08 & 0.14 & 0.23 \\
\hline & & Batch $100 \%$ & 0.02 & 0.11 & 0.27 & 0.21 & 0.15 & 0.14 & 0.12 & 0.13 & 0.22 & 0.17 & 0.10 & 0.10 & 0.25 & 0.27 \\
\hline & & No Batch & 0.02 & 0.14 & 0.30 & 0.18 & 0.20 & 0.36 & 0.35 & 0.36 & 0.24 & 0.21 & 0.08 & 0.12 & 0.12 & 0.18 \\
\hline & Optimal & Batch 50\% & 0.03 & 0.06 & 0.26 & 0.15 & 0.10 & 0.08 & 0.08 & 0.08 & 0.10 & 0.15 & 0.08 & 0.12 & 0.16 & 0.10 \\
\hline & & Batch $100 \%$ & 0.03 & 0.00 & 0.30 & 0.27 & 0.13 & 0.13 & 0.23 & 0.24 & 0.13 & 0.18 & 0.13 & 0.13 & 0.17 & 0.23 \\
\hline \multirow{6}{*}{$\underset{\sim}{\mathbb{N}}$} & & No Batch & 0.02 & 0.00 & 0.28 & 0.15 & 0.14 & 0.13 & 0.12 & 0.13 & 0.15 & 0.13 & 0.08 & 0.09 & 0.14 & 0.13 \\
\hline & Unoptimal & Batch $50 \%$ & 0.02 & 0.07 & 0.40 & 0.15 & 0.12 & 0.07 & 0.07 & 0.07 & 0.13 & 0.13 & 0.08 & 0.10 & 0.18 & 0.12 \\
\hline & & Butch $100 \%$ & 0.02 & 0.08 & 0.30 & 0.19 & 0.13 & 0.11 & 0.11 & 0.11 & 0.15 & 0.17 & 0.09 & 0.08 & 0.17 & 0.17 \\
\hline & & No Batch & 002 & 0.10 & 031 & 0.16 & 0.15 & 0.27 & 0.28 & 0.30 & 0.16 & 0.14 & 0.10 & 0.10 & 0.90 & 0.16 \\
\hline & Optimel & Batch $50 \%$ & 0.03 & 0.07 & 0.28 & 0.17 & 0.08 & 0.06 & 0.08 & 0.08 & 0.13 & 0.14 & 0.00 & 0.10 & 0.17 & 0.11 \\
\hline & & Batch $100 \%$ & 0.03 & 0.08 & 0.28 & 0.17 & 0.09 & 0.10 & 0.15 & 0.15 & 0.11 & 0.19 & 0.12 & 0.12 & 0.14 & 0.20 \\
\hline \multirow{6}{*}{$\stackrel{\sim}{\leftarrow}$} & & No Batch & 001 & 008 & 0.66 & 0.16 & 0.14 & 028 & 027 & 0.29 & 0.12 & 0.11 & 000 & 0.08 & 0.19 & 0.24 \\
\hline & UnoptImal & Batch $50 \%$ & 0.02 & 0.05 & 0.49 & 0.17 & 0.11 & 0.10 & 0.10 & 0.10 & 0.11 & 0.10 & 0.07 & 0.07 & 0.21 & 0.27 \\
\hline & & Batch $100 \%$ & 0.02 & 0.08 & 0.35 & 0.16 & 0.09 & 0.12 & 0.11 & 0.11 & 0.11 & 0.11 & 0.07 & 0.07 & 0.22 & 0.22 \\
\hline & & No Batch & 000 & 0.06 & 0.46 & 0.12 & 0.12 & 0.27 & 0.28 & 0.29 & 0.11 & 0.11 & 0.08 & 0.00 & 0.16 & 0.30 \\
\hline & Optimal & Batch $50 \%$ & 0.02 & 0.07 & 0.43 & 0.18 & 0.12 & 0.10 & 0.10 & 0.10 & 0.14 & 0.12 & 0.07 & 0.07 & 0.15 & 0.26 \\
\hline & & Batch $100 \%$ & 003 & 0.08 & 064 & 0.27 & 0.18 & 0.17 & 0.15 & 0.17 & 0.16 & 0.17 & 0.13 & 0.13 & 0.19 & 0.22 \\
\hline
\end{tabular}

\section{Demand Process: MA(1) $\theta:-\mathbf{0 . 2}$}

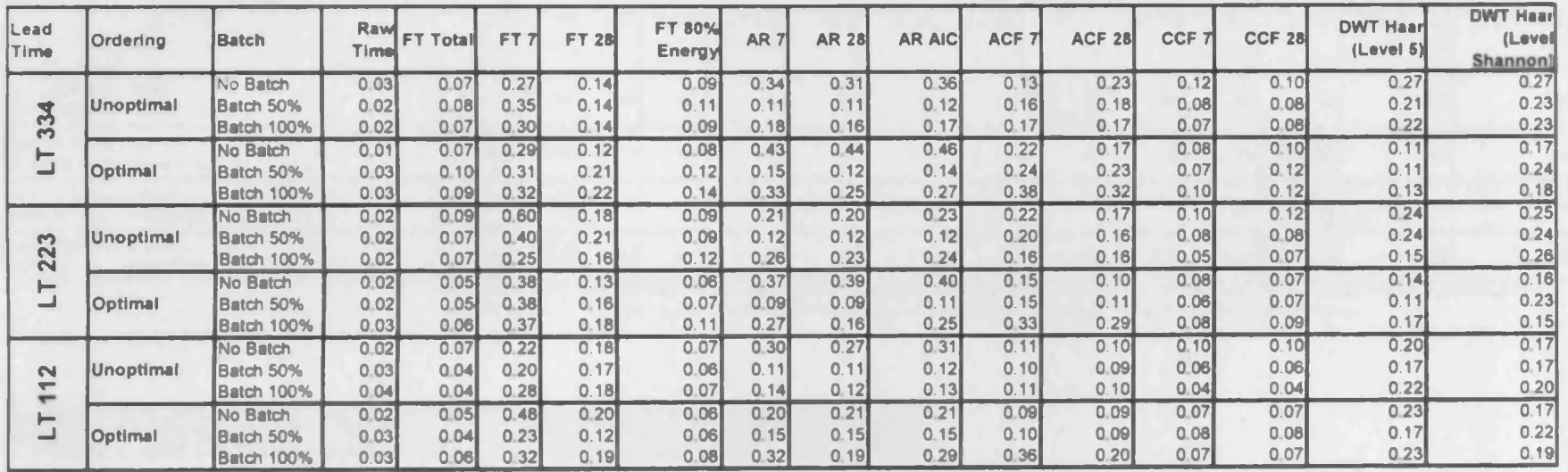

\section{Demand Process: MA(2) $\theta_{1}: 0.1 \theta_{2}:-0.8$}

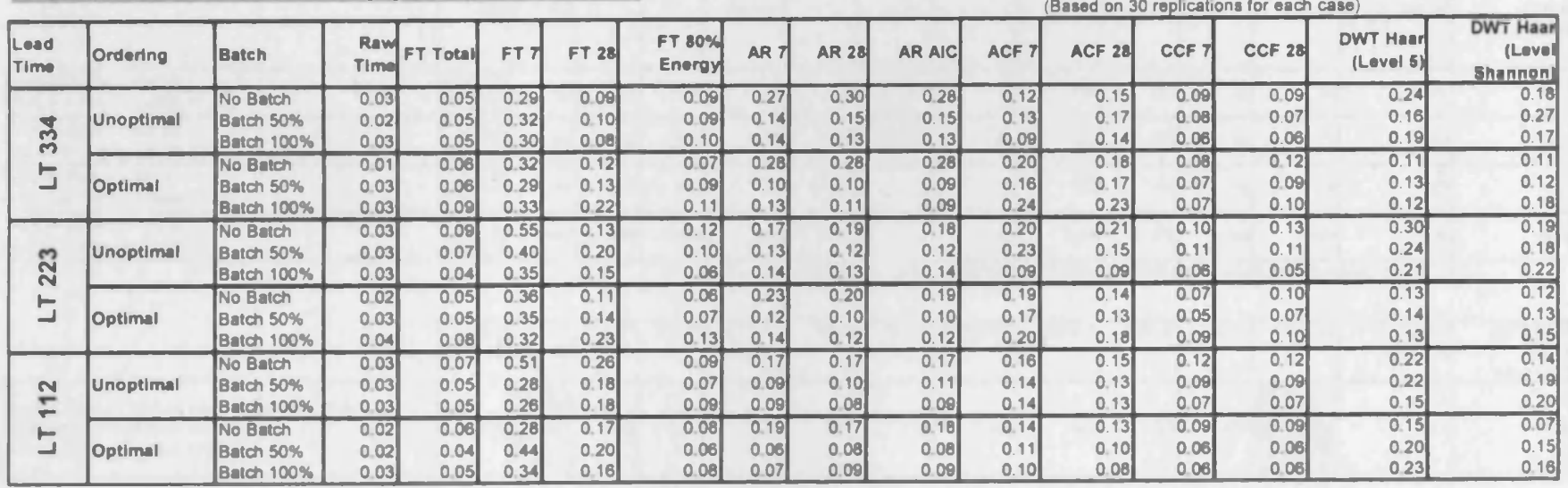




\section{Appendix $\mathbf{J}$ continued}

\section{J.3 Structured evaluation of feature parameter combinations used for index computation}

\begin{tabular}{|c|c|c|c|c|c|c|c|c|c|c|c|c|c|c|}
\hline \multirow{2}{*}{ Consistency Basis } & & & & & & & & & & \multicolumn{5}{|c|}{ Based on average rogue seasonaility index value } \\
\hline & $\begin{array}{l}\text { Ram } \\
\text { Time }\end{array}$ & $\begin{array}{ll}F T \\
\text { Total }\end{array}$ & FT7 & FT 28 & $\begin{array}{l}\text { FT } 80 \% \\
\text { Eneroy }\end{array}$ & AR 7 & $A R 28$ & AR AIC & ACF 7 & ACF 22 & CCF & CCF 2 ब & $\begin{array}{l}\text { DWT Hoert } \\
\text { (Level s) }\end{array}$ & $\begin{array}{l}\text { DWT Hoat } \\
\text { (Loval } \\
\text { shannon) }\end{array}$ \\
\hline \multicolumn{15}{|l|}{ Demand Process" } \\
\hline a) MA2 $\left(\theta_{1}, 01 \mathrm{H}_{2}-0.8\right)$ winh MA1 $(0.02$ & $2 / 18$ & $15 / 18$ & 7118 & $8 / 18$ & $16 / 18$ & $14 / 18$ & 1770 & $16 / 18$ & कातथ & $16 / 18$ & $5 / 18$ & 6/18 & $4 / 10$ & 010 \\
\hline b) $M A 1(0.0 .2)$ with $A R 2\left(p, 0.1 D_{2}-0.0\right)$ & $5 / 18$ & $14 / 18$ & 8/18 & 9/1 & $10 / 18$ & \&/18 & $8 / 18$ & $8 / 18$ & 19/19 & $19 / 18$ & 17118 & 1710 & 6/18 & $13 / 18$ \\
\hline Ordering process: Unoplimal whe oplmal for & & & & & & & & & & & & & & \\
\hline a) MA2 $\left(\theta, 0.1 \theta_{2}-0.8\right)$ & बत्ध & No & 390 & 200 & $5 / 10$ & $8 / 8$ & $6 / 8$ & 770 & 6/2 & $4 / 8$ & 40 & 4.9 & $5 / 9$ & 9: \\
\hline b) $M(1)(\theta-02)$ & कe⿻ & 60 & $4 / 9$ & 50 & 770 & $\overline{\text { जer }}$ & ज्ञात & 牙 & 779 & 76 & 69 & 5 की & 779 & का \\
\hline c) $A R 2\left(p_{1} 0.1 p_{2}-0.8\right)$ & 0.9 & 975 & $1 / 9$ & $5 / 9$ & We & $5 / 8$ & arg & $6 / 9$ & 때 & 28 & 770 & 70 & $8 / 9$ & 70 \\
\hline Overall Conessency - Demend and Ordering procese & 34/53 & 42/63 & 211633 & 2m63 & 47/6es & wes & 4N83 & 4N63 & sees & GeNes & 32163 & 39803 & 30183 & 3eves: \\
\hline
\end{tabular}

- for corresponding lead time (LT). ordering and batching combination ropeated for all combinations

\section{J.3.2 Rank analysis based on the magnitude of index}

(For assessing the discrimination ability of the index derived from different feature parameter combinations)

\begin{tabular}{|c|c|c|c|c|c|c|c|c|c|c|c|c|c|c|}
\hline & $\begin{array}{l}\text { REv } \\
\text { Time }\end{array}$ & $\begin{aligned} \text { FT } \\
\text { Total }\end{aligned}$ & FT 7 & FT 29 & $\begin{array}{l}\text { FT Bo\% } \\
\text { Energy }\end{array}$ & $A R=$ & कर26 & AR AIC & ACF 7 & ACF 29 & CCF & CCF 2D & $\begin{array}{l}\text { DWT Ranf } \\
\text { (Level 5) }\end{array}$ & $\begin{array}{l}\text { DWT Haen } \\
\text { (Level } \\
\text { shannon) }\end{array}$ \\
\hline $\begin{array}{l}\text { Sum of Ranks - MAN2) } \\
\text { Minimum / best possitle } 18\end{array}$ & 180 & 86 & 235 & 181 & 48 & 181 & 124 & 133 & 36 & 96 & 87 & 48 & 242 & 220 \\
\hline $\begin{array}{l}\text { Sum of Ranks - MA (1) } \\
\text { Minimum / beast posstble is }\end{array}$ & 151 & 82 & 218 & 154 & 62 & 173 & 175 & 176 & 62 & 110 & 53 & 28 & 242 & 190 \\
\hline $\begin{array}{l}\text { Sum of Ranks - AR (2) } \\
\text { Minimum I best possible } 18\end{array}$ & 154. & 78 & 234 & 143 & 75 & 142 & 184 & 158 & 61 & 96 & 80 & 50 & 239 & 217 \\
\hline $\begin{array}{l}\text { Sum of Ranks - TOTAL } \\
\text { Minim um / beet possible SA }\end{array}$ & 494 & 244 & 6 & 47: & 125 & 476 & 463 & 463 & 150 & 302 & 220 & $12 !$ & 723 & 138 \\
\hline
\end{tabular}

\section{7}

\section{J.3.3 Rank analysis based on the coefficient of index}

(For assessing the stochastic stability of the index derived from different feature parameter combinations)

\begin{tabular}{|c|c|c|c|c|c|c|c|c|c|c|c|c|c|c|}
\hline & $\begin{array}{l}\text { Ran } \\
\text { Time }\end{array}$ & $\begin{aligned} \text { FT } \\
\text { Total }\end{aligned}$ & FTत & क्रा 2ब & $\begin{array}{l}\text { FTso\% } \\
\text { Energy }\end{array}$ & ART & AR 2ब & AR AIC & ACF & ACF $2 \mathrm{C}$ & CCF & CCF 2द & $\begin{array}{l}\text { OWT Han! } \\
\text { (Lovel 5) }\end{array}$ & $\begin{array}{l}\text { DWT Han } \\
\text { (Leved } \\
\text { shannen) }\end{array}$ \\
\hline $\begin{array}{l}\text { Sum of Ranks - MAN21 } \\
\text { Minimum / bect possiblo } 18\end{array}$ & 18 & 37 & 251 & 174 & 86 & 159 & 152 & 154 & 176 & 168 & 68 & 79 & 194 & 173 \\
\hline $\begin{array}{l}\text { Sum of Ranks - MA (1) } \\
\text { Minimum / best possible } 18\end{array}$ & 18 & 41 & 234 & 158 & $\pi$ & 180 & 162 & 191 & 168 & 150 & 83] & 71 & 174 & 193 \\
\hline $\begin{array}{l}\text { Sum of Ranks - AR (2) } \\
\text { Minim um / best possible } 18\end{array}$ & 18 & 49 & 248 & 192 & 132 & 128 & 133 & 147 & 165 & 161 & 70 & 79. & 177 & 187 \\
\hline
\end{tabular}

\begin{tabular}{|c|c|c|c|c|c|c|c|c|c|c|c|c|c|c|}
\hline & $\begin{array}{l}\text { Ray } \\
\text { Timg }\end{array}$ & rot & FTन & FT 2E & $\begin{array}{l}\text { PT roosy } \\
\text { Eneray }\end{array}$ & ART & AR 2G & ARNG & ACF न & ACF 22 & CCF न & CCF 2I & $\begin{array}{l}\text { DWT Haa } \\
\text { (Level 5) }\end{array}$ & $\begin{array}{l}\text { DWT Ham } \\
\text { (Loval } \\
\text { shinnona) }\end{array}$ \\
\hline $\begin{array}{l}\text { sum of Ranks - TOTAL (magnitude as well as } \\
\text { coommerent of variation of the Index) } \\
\text { Minimum / best posesibio } 100\end{array}$ & 548 & 371 & 1422 & 1002 & 479 & M7! & 910 & 985 & 668 & 781 & 419 & 358 & 1268 & 1180 \\
\hline
\end{tabular}

\section{J.4 Correlation between alternative rogue seasonality indices}

\begin{tabular}{|l|r|r|r|}
\hline & Index and Alt 1 & Index and Alt 2 & Alt 1 and Alt \\
\hline Time & 0.69 & & 2 \\
FT & 0.93 & 0.85 & 0.33 \\
AR Model & 0.90 & 0.96 & 0.84 \\
ACF & 0.96 & 0.97 & 0.90 \\
CCF & 0.81 & 0.96 & 0.88 \\
DWT & 0.21 & 0.90 & 0.56 \\
\hline
\end{tabular}

Parameter options for each feature have been combined together

Highlighted indicates low correlation (considered bad)

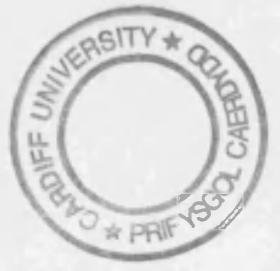

